







\section{Reaction Rate and Photochemical Data for Atmospheric Chemistry - 1977}

Edited by

Robert F. Hampson, Jr. and David Garvin

National Measurement Laboratory

National Bureau of Standards

Washington, D.C. 20234

Sponsored by the

Department of Transportation

National Aeronautics \& Space Administration

Office of Standard Reference Data, NBS

Office of Environmental Measurements, NBS

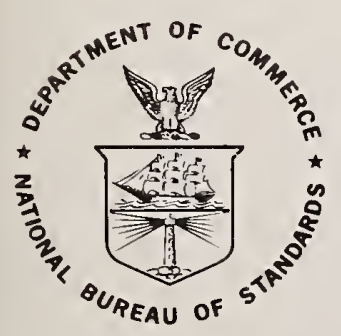

U.S. DEPARTMENT OF COMMERCE, Juanita M. Kreps, Secretary

Dr. Sidney Harman, Under Secretary

Jordan J. Baruch, Assistant Secretary for Science and Technology

s NATIONAL BUREAU OF STANDARDS, Ernest Ambler, Director 


\section{Library of Congress Cataloging in Publication Data}

Main entry under title:

Reaction rate and photochemical data for atmospheric chemistry, 1977.

(National Bureau of Standards special publication ; 513)

Supt. of Docs. no.: C13.10:513

1. Atmospheric chemistry--Tables. 2. Chemical reaction, Rate of-Tables, etc. 3. Photochemistry-Tables. I. Hampson, Robert F. 11. Garvin, David, 1923- III. United States. Dept. of Transportation IV. United States. National Bureau of Standards. V. Series: United States. National Bureau of Standards. Special publication ; 513 .

QC100.U57 no. 513 [QC879.6] 602'.1s [551.5'11] 78-606033

\section{National Bureau of Standards Special Publication 513}

Nat. Bur. Stand. (U.S.) Spec. Publ. 513, 111 pages (May 1978) CODEN: XNBSAV 
The National Standard Reference Data System was established in 1963 for the purpose of promoting the critical evaluation and dissemination of numerical data of the physical sciences. The program is coordinated by the Office of Standard Reference Data of the National Bureau of Standards, but involves the efforts of many groups in universities, government laboratories, and private industry. The primary aim of the program is to provide compilations of critically evaluated numerical data. These tables are published in the Journal of Physical and Chemical Reference Data, The NSRDS-NBS Publication Series of the National Bureau of Standards, and through other appropriate channels.

The present report consists of tables of data assembled for use in modelling the chemistry of the stratosphere. It represents contributions from the Chemical Kinetics Information Center, other NSRDS data centers, and a number of individual experts. Support for the preparation of those tables has been provided by the Department of Transportation under the High Altitude Pollution Program, by the National Aeronautics and Space Administration under the Upper Atmosphere Research Program, and by the Office of Standard Reference Data, N.B.S. and the Office of Environmental Measurements, N.B.S.

David R. Lide, Jr. Chief, office of Standard Reference Data 


\section{TABLE OF CONTENTS}

Abstract

1. Introduction

2. Guide to the Table

3. Reaction Index

4. Table. Data for chemical reactions and photochemistry of neutral species

5. Reference List

Appendices:

1. Chemical Thermodynamic Properties of Selected Molecular Species

2. Conversion Tables 
Reaction Rate and Photochemical Data for Atmospheric Chemistry $-1977^{*}$

R. F. Hampson, D. Garvin, Editors

A table of data for gas phase chemical reactions and photochemistry of neutral species is presented. Specifically, it gives preferred values for reaction rate constants, photoabsorption cross sections, and quantum yields of primary photochemical processes and also cites recent experimental work (to December 1977). It is intended to provide the basic physical chemical data needed as input data for calculations modelling atmospheric chemistry. An auxiliary table of thermochemical data for the pertinent chemical species is given in the appendix.

Key words: Air pollution; atmospheric chemistry; chemical kinetics; data evaluation; gas phase; photoabsorption cross section; photochemistry; quantum yield; rate constant.

\section{Introduction}

This technical note consists of a table of data on the kinetics of chemical reactions and the photochemistry of neutral species. It is designed for use in modelling the chemistry of the stratosphere and, to a more limited extent, the polluted troposphere and also the interpretation of laboratory experiments. The table of rate and photochemical data is supplemented by a table of thermochemical data for the species involved.

* This work was supported by the High Altitude Pollution Program of the Federal Aviation Administration, U. S. Department of Transportation, by the Upper Atmospheric Research Program of the National Aeronautics and Space Administration, and by the Office of Standard Reference Data, N.B.S. and the Office of Environmental Measurements, N.B.S. 
Earlier versions of this table have been issued as D. Garvin (editor) "Chemical Kinetics Data Survey IV" NBSIR 73-203 (1973); D. Garvin and R. F. Hampson (editors) "Chemical Kinetics Data Survey VII" NBSIR 74-430 (1974); and R. F. Hampson and D. Garvin (editors) "Chemical Kinetic and Photochemical Data for Modelling Atmospheric Chemistry" NBS Technical Note 866 (1975). The table appearing in NBS Technical Note 866 was also published in the U. S. Department of Transportation's Climatic Impact Assessment Program Monograph 1 "The Natural Stratosphere of 1974" E. Reiter, editor (1975). The present table supersedes all earlier versions.

Since the publication of NBS Technical Note 866 in 1975, a major effort in the evaluation of rate and photochemical data was undertaken for the NASA chlorofluoromethane assessment by its Laboratory Measurements Committee of which the present editors were members. The recommendations of this committee covering 104 chemical reactions and 48 photochemical processes are given in NASA Reference Publication 1010 "Chlorofluoromethanes and the Stratosphere" R. D. Hudson, editor, August 1977. These recommendations have been accepted by the editors of this table and are given here as preferred values. These recommendations are indicated either by the entry [**NASA(1977)eval] or by the symbols [†*] immediately preceding the author's name in the reference column. The first notation is used to indicate that the committee has synthesized a preferred value from a consideration of al1 data sets. The latter designation is used when the committee has accepted the results of a particular study or of an existing evaluation.

Recommended values also are given for reactions not considered by the NASA Laboratory Measurements Committee. Some of these are drawn from other modern rate data evaluations, and the sources are indicated in the table. Other recommendations have been prepared for this work.

Rate data evaluation is a small but growing branch of physical chemistry. There are more recommended rate constants than contained in this table, but it does contain most of those which are applicable to atmospheric chemistry. Evaluations and compilations of rate data and on-going programs in these areas have been described in the recent review paper "Evaluation and Compilation of Reaction Rate Data" by R. F. Hampson and D. Garvin, Journal of Physical Chemistry $81,2317-2319$ (1977). A current extensive 1isting of sources of evaluated rate data, NBS List of Publications 73 "Chemical Kinetics Tables, Data Evaluations and Bibliographies. A Guide to the Literature" is available on request from the NBS Chemical Kinetics Information Center.

Three sets of detailed data sheets have been published by us in the course of preparing these tables. They are R. F. Hampson (editor) "Survey of Photochemical and Rate Data for Twenty-eight Reactions of Interest in Atmospheric Chemistry" J. Phys. Chem. Ref. Data 2, 267 (1973); 
R. F. Hampson (editor) "Chemical Kinetics Data Survey VI, NBSIR 73-207 (1973); and D. Garvin (editor) "Chemical Kinetics Data Survey V" NBSIR 73-206 (1973), all recommendations contained in them (and still pertinent) are summarized in the present table.

We have also accepted many recommendations made by $R$. T. Watson in "Rate Constants for Reactions of $\mathrm{ClO}_{\mathrm{x}}$ of Atmospheric Interest" J. Phys. Chem. Ref. Data $\underline{6}, 871$ (1977) and by D. L. Baulch, et al "Evaluated Kinetic Data for High Temperature Reactions" Volume 1 (1972), Volume 2 (1973) and Volume 3 (1976), Butterworth and Co., London.

In addition to recommendations on rate and photochemical data the table includes listings of current research results. These data listings serve several purposes. Some simply record measurements, usually limited in number, on reactions for which it is not yet practical to give recommended values. Others show the data upon which a new recommendation is based. Still others show what has been done on a reaction since its rate constant was last evaluated. These new data may support the recommendation or suggest the need for modifications in the future.

\section{Guide to the Table}

\subsection{Genera1}

This table provides current (December 1977) information on reaction rate constants, quantum yields and absorption cross sections. For many reactions, preferred values are given. The reactions included in the table are summarized in the index of reactions given in section 3 .

Most of the important stratospheric reactions are in the tables. A number of the less important ones and some related systems are included, of ten simply for comparison.

The following remarks summarize the content of the table. It lists 416 reactions and gives a preferred value of the rate constant for 194 reactions; 136 based on recent reviews and 58 based on recent (1975-1977) experimental work. New data entries (1975-1977) are given for 252 reactions, showing substantial activity in the study of gas kinetics.

The following principles have been used in the selection and presentation of data:

a. Where a preferred value is given for a rate constant or photochemical quantity, it is so indicated by an asterisk [*] placed ahead of the entry in the reference column.

b. As indicated earlier an asterisk preceded by a dagger symbol [ $\dagger^{*}$ ] is used to indicate those preferred values that have been recommended by the NASA Laboratory Measurements Committee and published in NASA Reference Publication 1010 "Chlorofluoromethanes and the Stratosphere" R. D. Hudson, editor, August 1977. 
c. Where there is a recent published evaluation of the data and there are no newer data, the evaluation is adopted and usually marked with an asterisk. The original data covered by the evaluation are not listed separately. However this latter rule has not been applied when the evaluation has appeared within the past year.

d. Where there is an evaluation but there are new data, both are listed and a preferred value is indicated (marked by an asterisk).

e. Where desirable, the available recent data sets are listed, and a selection is made or a preferred value is synthesized from them.

\subsection{Uncertainty in Recommended Value of a Rate Constant}

The uncertainty assigned here to the recommended value of a rate constant is given in the column "Notes and reliability of $\log \mathrm{k}^{\prime \prime}$. This is an estimate by the evaluator of the absolute accuracy of the preferred value. It is a subjective judgment derived from intercomparison of data sets, consideration of related reactions studied with the same technique, estimates of how well the parameters could have been controlled, and comparison with theory. It means that in the evaluators judgment, the true value will lie within the indicated limits to a high level of confidence (90 to 95 percent).

Usually the uncertainty is indicated by the term $D$ in the expression: $\log _{10} \mathrm{k}=\mathrm{C} \pm \mathrm{D}$. This is equivalent to the statement that $k$ is uncertain to a factor of $F$ where $D=\log _{10} F$. The statement that $k$ has the value $k_{0}$ and is uncertain to a factor of $\mathrm{F}$ means that $\mathrm{k}_{\mathrm{o}} / \mathrm{F}<\mathrm{k}<\mathrm{k}_{\mathrm{o}} \mathrm{F}$.

Other forms used to indicate reliability are the following:

$\mathrm{A}<\mathrm{k}<\mathrm{B}$ means $\mathrm{k}$ lies in the range between $\mathrm{A}$ and $\mathrm{B}$

$k<(>)$ B means $B$ is an upper (lower) limit

$k \sim B$ means $B$ is only a rough guide to value of $k$

$k=A \pm B$ alternative form for stating reliability limits 
At times a rate constant expression is quoted from a paper with individual uncertainties for the rate parameters as $k=$ $(A \pm a) \exp (-B \pm b / T)$. These measures are those provided by the author and often are indications of precision, not overall reliability.

Some notes are in order, regarding the statement of the uncertainty in the recommended value of the rate constant for those recommended values recommended by the NASA Laboratory Measurements Committee. Those recommendations were made for the limited temperature range $200-300 \mathrm{~K}$. The uncertainty is indicated for $\mathrm{T}=230 \mathrm{~K}$, a typical midstratospheric temperature and in some cases allowance for extrapolation from higher temperatures was necessary. This is why occasionally the entry consists of the value of a rate constant measured at $298 \mathrm{~K}$, the symbols [ ${ }^{+*}$ ] immediately ahead of the entry in the reference column to show that this value has been adopted by the Committee for the temperature range $200-300 \mathrm{~K}$, and a statement of the uncertainty in the preferred value at $T=230 \mathrm{~K}$. This stated uncertainty will generally be larger than the uncertainty in the measured value (measured at $298 \mathrm{~K}$ ) to allow for the additional uncertainty introduced by extrapolation to $\mathrm{T}=230 \mathrm{~K}$.

For the recommendations made by the NASA Laboratory Committee the indicated uncertainties in NASA Reference Publication 1010 have been doubled in the present table in order to make the reliability statement analogous to a "95 percent confidence level".

\subsection{Conventions Concerning Rate Constants}

a. General Convention. Almost all of the reactions in the table are elementary processes. For them the rate expression is derivable from a statement of the reaction, e.g.

$$
\begin{gathered}
A+2 B \rightarrow A B_{2} \\
-d[A] / d t=-(1 / 2) d[B] / d t=d\left[A B_{2}\right] / d t=k[A][B]^{2}
\end{gathered}
$$

Note that the stoichiometric coefficient for B, i.e. 2, appears in the denominator before $B^{\prime} s$ rate of change (which is equal to $2 \mathrm{k}[\mathrm{A}][\mathrm{B}]^{2}$ ) and as a power on the right hand side.

Wherever there may be any doubt an explicit rate expression is given. 
b. Dissociation and Combination Reactions. Some reactions of these types are not of integral kinetic order over the stratospheric pressure and temperature range. That is, although they require an energy transfer agent, " $M$," they are in the "pressure fall-off region". For some such reactions we tabulate rate constants that include the effect of the energy transfer agent and give rates for various altitudes. The reactions are written to demphasize "M," e.g.,

$$
\mathrm{NO}_{2}+\mathrm{NO}_{3}(+\mathrm{M}) \rightarrow \mathrm{N}_{2} \mathrm{O}_{5}(+\mathrm{M})
$$

$$
\mathrm{HNO}_{3} \stackrel{\mathrm{M}}{\rightarrow} \mathrm{HO}+\mathrm{NO}_{2}
$$

The rate expressions to be used do not have the concentration of $M$ in them, e.g., Rate $=k\left[\mathrm{NO}_{2}\right]\left[\mathrm{NO}_{3}\right]$ and $\mathrm{k}\left[\mathrm{HNO}_{3}\right]$ respectively. The units given for the $\mathrm{k}^{\prime} \mathrm{s}, \mathrm{s}^{-1}$ for first order, and $\mathrm{cm}^{+3}$ molecule $\mathrm{e}^{-1} \mathrm{~s}^{-1}$ for second order, are consistent with this convention.

The altitude, temperature and number density regime used in these cases is

$\begin{array}{ccc}\text { altitude/km } & \text { temp./K } & \log [\mathrm{M}] / \mathrm{molecu} \\ & & \\ 15 & 217 & 18.60 \\ 20 & 217 & 18.27 \\ 25 & 222 & 17.93 \\ 30 & 227 & 17.58 \\ 35 & 237 & 17.26 \\ 40 & 251 & 16.92 \\ 45 & 265 & 16.60\end{array}$

This table follows the U. S. standard atmosphere, 1976.

c. Forward and Reverse Reactions. In some cases there are no data on a reaction of interest but there are data on the "reverse" reaction. Occasionally, for

$$
\begin{aligned}
& A \rightarrow B(f) \\
& B \rightarrow A(r)
\end{aligned}
$$

an evaluation will use the data on the "reverse" reaction together with an equilibrium constant to obtain the other rate constant. Obviously this is an approximation but it often is a useful method of estimating non-measured physical properties. 
The table contains notations to warn the reader when this procedure has been used, such as "based on reverse reaction," or when both reactions $\underline{\underline{f}}$ and $\underline{\mathrm{r}}$ are listed together " $k_{f}=k_{r} K_{e q}$ " or $\mathrm{k}_{\mathrm{r}}=\mathrm{k}_{\mathrm{f}} / \mathrm{k}_{\mathrm{eq}}$. These expressions, which are those used in the analyses, are based on equating the two rates at equilibrium.

$$
\begin{gathered}
R_{f}=R_{r} \\
k_{f}[A]=k_{r}[B] \\
k_{e q}=[B] /[A]=k_{f} / k_{r} .
\end{gathered}
$$

2.4 Convention Concerning Optical Absorption Coefficients

These are reported in the table as "absorption cross sections per molecule, base $e^{\prime \prime}$. They are defined by the equations.

$$
\begin{gathered}
I / I_{0}=\exp (-\sigma[N] \ell) \\
\sigma=(1 /([N] \ell)) \ln \left(I_{0} / I\right)
\end{gathered}
$$

where $I_{0}$ and $I$ are the intensities of incident and transmitted light, $\sigma$ is the absorption cross section, $\mathrm{cm}^{2}$ molecule $\mathrm{e}^{-1},[\mathrm{~N}]$ is the concentration of absorbers, molecules $\mathrm{cm}^{-3}$, and $l$ is the path length, $\mathrm{cm}$. Other definitions and units are frequently used. The terms "absorption coefficient" and "extinction coefficient" are common. It is always necessary to know what concentration units, path length units and type of logarithm (base e or base 10) are used in the definition. To convert "cross-sections" to absorption coefficients in $(\text { atm at } 273 \mathrm{~K})^{-1} \mathrm{~cm}^{-1}$, base e, multiply by $2.69 \times 10^{19}$.

A table of conversion factors is given in the appendix. 


\section{Arrangement of the Table}

Data on a reaction appear only once in the table. The normal location for a reaction is determined by its reactants. Each species has been assigned a sequence number ( 1 to 65 ) as shown in the index that follows. These sequence numbers are the same as those used in NBS Technical Note 866. New species have been inserted in appropriate positions and assigned sequence numbers such as $34 \mathrm{a}, 34 \mathrm{~b}$, etc. A reaction is filed under the lower numbered species. That is, the reaction of ozone (7) with an oxygen atom ( 1 ) is filed under reactions of oxygen atoms. The numbers for the reactants appear at the left margin of the table, preceding the statement of the reaction, e.g. $1,70+\mathrm{O}_{3} \rightarrow \mathrm{O}_{2}+\mathrm{O}_{2}$. These number pairs run in ascending order through the table.

If a reaction is not filed in the location described above, a cross reference is given there. The most common exception is the listing together of data on the forward and reverse reactions of a pair, i.e. $A \rightarrow B, B \rightarrow A$.

In the index that follows, bimolecular reactions are listed under both reactants. Frequently the listing of reaction partners for a particular species is divided into two parts by three dashes, separating species earlier in the list than the species indexed from those later in the list. Reactions of the particular species with those species listed before the three dashes should be sought under those reaction partners. There is no indexing of products of reactions. A few species are listed in the index for which there are no reactions in the table, in anticipation of expansion of the data set. 
Rxn with: $\mathrm{O}, \mathrm{O}\left({ }^{1} \mathrm{~S}\right), \mathrm{O}_{2}, \mathrm{O}_{3}, \mathrm{~N}, \mathrm{NO}, \mathrm{NO}_{2}, \mathrm{NO}_{3}, \mathrm{~N}_{2}, \mathrm{~N}_{2} \mathrm{O}$, $\mathrm{N}_{2} \mathrm{O}_{5}, \mathrm{NH}_{2}, \mathrm{NH}_{3}, \mathrm{H}, \mathrm{HO}, \mathrm{HO}_{2}, \mathrm{H}_{2}, \mathrm{H}_{2} \mathrm{O}, \mathrm{H}_{2} \mathrm{O}_{2}$, $\mathrm{HNO}_{2}, \mathrm{HNO}_{3}, \mathrm{SO}, \mathrm{SO}_{2}, \mathrm{SO}_{3}, \mathrm{HS}, \mathrm{H}_{2} \mathrm{~S}, \mathrm{CS}, \mathrm{CS}_{2}$, OCS, ClO, BrO, OClO, $\mathrm{HCl}, \mathrm{HBr}, \mathrm{NO}_{3} \mathrm{Cl}, \mathrm{Cl}_{2}$, $\mathrm{Cl}_{2} \mathrm{O}, \mathrm{CO}, \mathrm{CO}_{2}, \mathrm{CN}, \mathrm{CHO}, \mathrm{CH}_{2} \mathrm{O}, \mathrm{CH}_{3}, \mathrm{CH}_{3} \mathrm{NO}_{2}$, $\mathrm{CH}_{3} \mathrm{ONO}, \mathrm{CH}_{4}, \mathrm{C}_{2} \mathrm{H}_{4}, \mathrm{C}_{2} \mathrm{H}_{6}, \mathrm{C}_{3} \mathrm{H}_{6}$, alkane, $\mathrm{C}_{6} \mathrm{H}_{6}, \mathrm{C}_{6} \mathrm{H}_{5} \mathrm{CH}_{3}, \mathrm{CH}_{3} \mathrm{Cl}$

2. $O\left({ }^{I_{D}}\right)$ Rxn with: $\mathrm{O}_{2}, \mathrm{O}_{3}, \mathrm{NO}, \mathrm{NO}_{2}, \mathrm{~N}_{2}, \mathrm{~N}_{2} \mathrm{O}, \mathrm{NH}_{3}, \mathrm{H}_{2}, \mathrm{H}_{2} \mathrm{O}$, $\mathrm{H}_{2} \mathrm{O}_{2}, \mathrm{HCl}, \mathrm{CO}_{2}, \mathrm{CH}_{4}, \mathrm{C}_{2} \mathrm{H}_{6}, \mathrm{CF}_{2} \mathrm{Cl}_{2}, \mathrm{CFCl}_{3}$

3. $\mathrm{O}\left({ }^{1} \mathrm{~S}\right)$ Rxn with: $\mathrm{O}, \mathrm{O}_{2}, \mathrm{O}_{3}, \mathrm{NO}, \mathrm{NO}_{2}, \mathrm{~N}_{2}, \mathrm{~N}_{2} \mathrm{O}, \mathrm{NH}_{3}, \mathrm{H}_{2} \mathrm{O}$, $\mathrm{CO}_{2}, \mathrm{CH}_{4}$

4. $\mathrm{O}_{2}$

Rxn with: $0, O\left({ }^{1}\right), O\left({ }^{1} S\right) \ldots$ $h \nu, \mathrm{N}, \mathrm{NO}, \mathrm{NO}_{2}, \mathrm{NO}_{3}, \mathrm{~N}_{2}, \mathrm{H}, \mathrm{H}_{2}, \mathrm{H}_{2} \mathrm{O}, \mathrm{H}_{2} \mathrm{O}_{2}$, HNO, $\mathrm{SO}, \mathrm{HS}, \mathrm{Cl}, \mathrm{CN}, \mathrm{CHO}, \mathrm{CH}_{3}, \mathrm{CH}_{3} \mathrm{O}$

5. $\mathrm{O}_{2}\left({ }^{I} \Delta\right)$ Rxn with: $\mathrm{O}_{2}, \mathrm{O}_{3}, \mathrm{~N}, \mathrm{NO}, \mathrm{N}_{2}, \mathrm{H}, \mathrm{SO}, \mathrm{SO}_{2}, \mathrm{H}_{2} \mathrm{~S}, \mathrm{CO}$, $\mathrm{CF}_{2} \mathrm{Cl}_{2}, \mathrm{CCl}_{4}, \mathrm{CH}_{3} \mathrm{Cl}, \mathrm{CH}_{2} \mathrm{Cl}_{2}, \mathrm{CHCl}_{3}$

6. $\mathrm{O}_{2}\left({ }^{{ }} \Sigma\right)$ Rxn with: $\mathrm{O}_{2}, \mathrm{~N}_{2}, \mathrm{H}_{2} \mathrm{O}$

7. $\mathrm{O}_{3} \quad$ Rxn with: $O, O\left({ }^{1} \mathrm{D}\right), \mathrm{O}\left({ }^{1} \mathrm{~S}\right), \mathrm{O}_{2}\left({ }^{1} \Delta\right)-\ldots$ hv, M, N, NO, $\mathrm{NO}_{2}, \mathrm{H}, \mathrm{HO}, \mathrm{HO}_{2}, \mathrm{SO}, \mathrm{SO}_{2}$, $\mathrm{H}_{2} \mathrm{~S}, \mathrm{Cl}, \mathrm{Br}, \mathrm{ClO}, \mathrm{BrO}, \mathrm{CO}, \mathrm{CH}_{2} \mathrm{O}, \mathrm{CH}_{3}$, $\mathrm{CH}_{3} \mathrm{O}_{2}, \mathrm{CH}_{3} \mathrm{ONO}, \mathrm{CH}_{4}, \mathrm{C}_{2} \mathrm{H}_{4}, \mathrm{C}_{3} \mathrm{H}_{6}$, allene, butenes, butadiene, $\mathrm{C}_{2} \mathrm{Cl}_{2} \mathrm{H}_{2}$ 
8. N

9. NO

10. $\mathrm{NO}_{2}$

11. $\mathrm{NO}_{3}$

12. $\mathrm{N}_{2}$

13. $\mathrm{N}_{2} \mathrm{O}$

14. $\mathrm{N}_{2} \mathrm{O}_{5}$

15. $\mathrm{NH}$

16. $\mathrm{NH}_{2}$

17. $\mathrm{NH}_{3}$
Rxn with: $O, O_{2}, O_{2}\left({ }^{1} \Delta\right), O_{3} \ldots$

$\mathrm{N}, \mathrm{NO}, \mathrm{NO}_{2}, \mathrm{HO}, \mathrm{SO}, \mathrm{SO}_{3}, \mathrm{OClO}$

Rxn with: $O, O\left({ }^{1} D\right), O\left({ }^{1} S\right), O_{2}, O_{2}\left({ }^{1} \Delta\right), O_{3}, N-\cdots$ hv, $\mathrm{M}, \mathrm{NO}, \mathrm{NO}_{2}+\mathrm{H}_{2} \mathrm{O}, \mathrm{NO}_{3}, \mathrm{NH}, \mathrm{NH}_{2}, \mathrm{H}, \mathrm{HO}$, $\mathrm{HO}_{2}, \mathrm{H}_{2}, \mathrm{H}_{2} \mathrm{O}, \mathrm{H}_{2} \mathrm{O}_{2}, \mathrm{Cl}, \mathrm{ClO}, \mathrm{BrO}, \mathrm{OClO}$, $\mathrm{CH}_{3}, \mathrm{CH}_{3} \mathrm{O}, \mathrm{CH}_{3} \mathrm{O}_{2}$

Rxn with: $O, O\left({ }^{1} D\right), O\left({ }^{1} S\right), O_{2}, O_{3}, N, N O+\mathrm{H}_{2} \mathrm{O} \ldots$ hv, M, $\mathrm{NO}_{2}, \mathrm{NO}_{3}, \mathrm{NH}_{2}, \mathrm{NH}_{3}, \mathrm{H}, \mathrm{HO}, \mathrm{HO}_{2}$, $\mathrm{SO}_{2}, \mathrm{Cl}, \mathrm{ClO}, \mathrm{CH}_{3}, \mathrm{CH}_{3} \mathrm{O}, \mathrm{CH}_{3} \mathrm{O}_{2}$

Rxn with: $\mathrm{O}, \mathrm{O}_{2}, \mathrm{NO}, \mathrm{NO}_{2}-\cdots$

hv, $\mathrm{M}, \mathrm{NO}_{3}, \mathrm{H}_{2} \mathrm{O}, \mathrm{SO}_{2}$

Rxn with: $O, O\left({ }^{1} D\right), O\left({ }^{1} S\right), O_{2}, O_{2}\left({ }^{1} \Delta\right), O_{2}\left({ }^{I} \Sigma\right) \cdots$ $\mathrm{M}, \mathrm{HO}$

Rxn with: $O, O\left({ }^{1} D\right), O\left({ }^{1} S\right) \ldots$

$h \nu, M, H, H O, C l O, C O$

Rxn with: $0 \ldots h \nu, \mathrm{M}, \mathrm{H}_{2} \mathrm{O}, \mathrm{SO}_{2}$

Rxn with: NO - -

Rxn with: $\mathrm{O}$, NO $-\mathrm{H}, \mathrm{HO}, \mathrm{H}_{2}, \mathrm{H}_{2} \mathrm{O}$

Rxn with: $O, O\left({ }^{1} D\right), O\left({ }^{1} S\right), \mathrm{NO}_{2} \cdots$

$\mathrm{M}, \mathrm{H}, \mathrm{HO}, \mathrm{C} 10$ 
17a. $\mathrm{N}_{2} \mathrm{H}_{4}$

18. $\mathrm{H}$

19. HO

20. $\mathrm{HO}_{2}$

21. $\mathrm{H}_{2}$

22. $\mathrm{H}_{2} \mathrm{O}$

2

\section{Rxn with: $\mathrm{H}$}

Rxn with: $\mathrm{O}, \mathrm{O}_{2}, \mathrm{O}_{2}\left({ }^{2} \Delta\right), \mathrm{O}_{3}, \mathrm{NO}, \mathrm{NO}_{2}, \mathrm{~N}_{2} \mathrm{O}, \mathrm{NH}_{2}, \mathrm{NH}_{3}$, $\mathrm{N}_{2} \mathrm{H}_{4}$ - -

$\mathrm{H}, \mathrm{HO}, \mathrm{HO}_{2}, \mathrm{H}_{2} \mathrm{O}, \mathrm{H}_{2} \mathrm{O}_{2}, \mathrm{HNO}, \mathrm{HNO}_{2}, \mathrm{HNO}_{3}$,

$\mathrm{SO}_{2}, \mathrm{HS}, \mathrm{H}_{2} \mathrm{~S}, \mathrm{COS}, \mathrm{OClO}, \mathrm{HCl}, \mathrm{NOCl}, \mathrm{Cl}_{2}, \mathrm{CO}$,

$\mathrm{CO}_{2}, \mathrm{CH}_{2} \mathrm{O}, \mathrm{CH}_{3} \mathrm{OOH}, \mathrm{CH}_{3} \mathrm{ONO}, \mathrm{CH}_{3} \mathrm{Cl}$

Rxn with: $\mathrm{O}, \mathrm{O}_{3}, \mathrm{~N}, \mathrm{NO}, \mathrm{NO}_{2}, \mathrm{~N}_{2}, \mathrm{~N}_{2} \mathrm{O}, \mathrm{NH}_{2}, \mathrm{NH}_{3}, \mathrm{H}-\cdots$ $\mathrm{M}, \mathrm{HO}, \mathrm{HO}_{2}, \mathrm{H}_{2}, \mathrm{D}_{2}, \mathrm{H}_{2} \mathrm{O}, \mathrm{H}_{2} \mathrm{O}_{2}$, $\mathrm{HNO}, \mathrm{HNO}_{2}$, $\mathrm{HNO}_{3}, \mathrm{SO}_{2}, \mathrm{H}_{2} \mathrm{~S}, \mathrm{Cl}, \mathrm{HCl}, \mathrm{DCl}, \mathrm{HBr}, \mathrm{NO}_{3} \mathrm{Cl}$, $\mathrm{CO}, \mathrm{CH}_{2} \mathrm{O}, \mathrm{CH}_{3} \mathrm{OH}, \mathrm{CH}_{3} \mathrm{NO}_{2}, \mathrm{CH}_{3} \mathrm{ONO}, \mathrm{CH}_{4}, \mathrm{C}_{2} \mathrm{H}_{2}$, $\mathrm{C}_{2} \mathrm{H}_{4}, \mathrm{C}_{2} \mathrm{H}_{6}, \mathrm{C}_{3} \mathrm{H}_{6}, \mathrm{C}_{4} \mathrm{H}_{10}$, alkane, $\mathrm{C}_{6} \mathrm{H}_{6}$, $\mathrm{C}_{6} \mathrm{H}_{5} \mathrm{CH}_{3}, \mathrm{CF}_{2} \mathrm{Cl}_{2}, \mathrm{CFCl}_{3}, \mathrm{CCl}_{4}, \mathrm{CH}_{3} \mathrm{Cl}$, $\mathrm{CH}_{2} \mathrm{Cl}_{2}, \mathrm{CHCl}_{3}, \mathrm{CH}_{i} \mathrm{Cl}_{j} \mathrm{~F}_{k}$

Rxn with: $\mathrm{O}, \mathrm{O}_{3}, \mathrm{NO}, \mathrm{NO}_{2}, \mathrm{H}, \mathrm{HO}-\ldots$ $\mathrm{M}, \mathrm{HO}_{2}, \mathrm{H}_{2}, \mathrm{H}_{2} \mathrm{O}, \mathrm{SO}_{2}, \mathrm{Cl}, \mathrm{Br}, \mathrm{ClO}, \mathrm{CO}, \mathrm{CH}_{2} \mathrm{O}$, $\mathrm{CH}_{3} \mathrm{O}_{2}, \mathrm{C}_{2} \mathrm{H}_{4}, \mathrm{C}_{2} \mathrm{H}_{6}, \mathrm{C}_{3} \mathrm{H}_{8}, \mathrm{C}_{4}{ }^{\mathrm{H}} 10$

Rxn with: $\mathrm{O}, \mathrm{O}\left({ }^{1} \mathrm{D}\right), \mathrm{O}_{2}, \mathrm{NO}, \mathrm{NH}_{2}, \mathrm{HO}, \mathrm{HO}_{2}-\cdots$ $\mathrm{M}, \mathrm{Cl}, \mathrm{ClO}$

Rxn with: $\left.O, O\left({ }^{1} \mathrm{D}\right), \mathrm{O}^{1} \mathrm{~S}\right), \mathrm{O}_{2}, \mathrm{O}_{2}\left({ }^{1} \Sigma\right)$, NO, $\mathrm{NO}_{3}, \mathrm{~N}_{2} \mathrm{O}_{5}$, $\mathrm{NH}_{2}, \mathrm{H}, \mathrm{HO}, \mathrm{HO}_{2}-\cdots \mathrm{SO}_{3}$

23. $\mathrm{H}_{2} \mathrm{O}_{2}$ Rxn with: $\left.\mathrm{O}, \mathrm{O}^{1}{ }^{1} \mathrm{D}\right), \mathrm{O}_{2}, \mathrm{NO}, \mathrm{H}, \mathrm{HO}-$. $h \nu, M, C l, B r$ 
24. HNO

Rxn with: $\mathrm{O}_{2}, \mathrm{H}, \mathrm{HO}-\ldots \mathrm{M}$, HNO

25. $\mathrm{HNO}_{2}$

Rxn with: O, H, HO - - hv

26. $\mathrm{HNO}_{3}$

Rxn with: O, H, HO - - hv, M, Cl

26a. s

Rxn with: $\mathrm{CS}_{2}, \cos$

27. So

Rxn with: $\mathrm{O}, \mathrm{O}_{2}, \mathrm{O}_{2}\left({ }^{1} \Delta\right), \mathrm{O}_{3}, \mathrm{~N}-\cdots \mathrm{SO}, \mathrm{SO}_{3}$

28. $\mathrm{SO}_{2}$

Rxn with: $\mathrm{O}, \mathrm{O}_{2}\left({ }^{1} \Delta\right), \mathrm{O}_{3}, \mathrm{NO}_{2}, \mathrm{NO}_{3}, \mathrm{~N}_{2} \mathrm{O}_{5}, \mathrm{H}, \mathrm{HO}$,

$$
\mathrm{HO}_{2}--\mathrm{CH}_{3}
$$

29. $\mathrm{SO}_{3}$

Rxn with: $\mathrm{O}, \mathrm{N}, \mathrm{H}_{2} \mathrm{O}, \mathrm{SO}-\ldots$

30. $\mathrm{S}_{2} \mathrm{O}$

Rxn with:

31. HS

Rxn with: $\mathrm{O}, \mathrm{O}_{2}, \mathrm{H}-\cdots \mathrm{HS}$

32. $\mathrm{H}_{2} \mathrm{~S}$

Rxn with: $\mathrm{O}, \mathrm{O}_{2}\left({ }^{1} \Delta\right), \mathrm{O}_{3}, \mathrm{H}, \mathrm{HO}-\cdots$

33. $\mathrm{HSO}_{3}$

Rxn with:

34. $\mathrm{H}_{2} \mathrm{SO}_{4}$

Rxn with:

$34 a \cdot C S$

Rxn with: 0 - -

$34 \mathrm{~b} \cdot \mathrm{CS}_{2}$

Rxn with: $0, S_{-}-$

$34 c \cdot \cos$

Rxn with: $0, \mathrm{H}, \mathrm{S}-\ldots$ 
35. $\mathrm{Cl}$

Rxn with: $\mathrm{O}_{2}, \mathrm{O}_{3}, \mathrm{NO}, \mathrm{NO}_{2}, \mathrm{HO}, \mathrm{HO}_{2}, \mathrm{H}_{2}, \mathrm{H}_{2} \mathrm{O}_{2}$, $\mathrm{HNO}_{3}-\mathrm{Cl}_{2} \mathrm{OClO}, \mathrm{ClOO}, \mathrm{NOCl}, \mathrm{NO}_{2} \mathrm{Cl}$, $\mathrm{NO}_{3} \mathrm{Cl}, \mathrm{Cl}_{2} \mathrm{O}, \mathrm{CH}_{4}, \mathrm{C}_{2} \mathrm{H}_{6}$

$\mathrm{Br}$

Rxn with: $\mathrm{O}_{3}, \mathrm{HO}_{2}, \mathrm{H}_{2} \mathrm{O}_{2}-\cdots$

36. $\quad$ C10

Rxn with: $\mathrm{O}, \mathrm{O}_{3}, \mathrm{NO}, \mathrm{NO}_{2}, \mathrm{~N}_{2} \mathrm{O}, \mathrm{NH}_{3}, \mathrm{HO}_{2}, \mathrm{H}_{2}-\cdots$ hv, $\mathrm{ClO}, \mathrm{BrO}, \mathrm{CO}, \mathrm{CH}_{4}, \mathrm{C}_{2} \mathrm{H}_{2}, \mathrm{C}_{2} \mathrm{H}_{4}$

Bro Rxn with: $0, \mathrm{O}_{3}$, NO, ClO - - Bro

37. Cl00 Rxn with: Cl - - hv, M

OClO Rxn with: O, N, NO, H, Cl - - hv

38. $\mathrm{ClO}_{3}$ Rxn with:

39. $\mathrm{HCl}$ Rxn with: O, O $\left({ }^{1} \mathrm{D}\right), \mathrm{H}, \mathrm{HO}-\cdots \mathrm{hv}$

HBr Rxn with: O, HO - -

40. HOCl Rxn with: hv

41. HOClO Rxn with:

42. NOCl Rxn with: H, Cl - - hv

43. $\mathrm{NO}_{2} \mathrm{Cl}$ Rxn with: $\mathrm{Cl}-\ldots$ hv

43a. $\mathrm{NO}_{3} \mathrm{Cl}$ Rxn with: $\mathrm{O}, \mathrm{HO}, \mathrm{Cl}-\mathrm{hu}^{-}$

44. $\mathrm{Cl}_{2}$ Rxn with: $\mathrm{O}, \mathrm{H}-\cdots$ hv

44a. $\mathrm{Cl}_{2} \mathrm{O}$ Rxn with: $\mathrm{O}, \mathrm{Cl}-\cdots$ 
45. CO

Rxn with: $\mathrm{O}, \mathrm{O}_{2}\left({ }^{\mathrm{I}} \Delta\right), \mathrm{O}_{3}, \mathrm{~N}_{2} \mathrm{O}, \mathrm{H}, \mathrm{HO}, \mathrm{HO}_{2}, \mathrm{ClO}-$

$$
\mathrm{CH}_{3} \mathrm{O}
$$

46. $\mathrm{CO}_{2}$

Rxn with: $O, O\left({ }^{I} D\right), O\left({ }^{I} S\right), H-\cdots M$

46a. $\mathrm{CN}$

Rxn with: $\mathrm{O}, \mathrm{O}_{2}-\cdots$

47. $\mathrm{CHO}$

Rxn with: $\mathrm{O}, \mathrm{O}_{2}-\cdots$

48. $\mathrm{CH}_{2} \mathrm{O}$

Rxn with: $\mathrm{O}, \mathrm{O}_{3}, \mathrm{H}, \mathrm{HO}, \mathrm{HO}_{2}-\cdots$ hv

49. $\mathrm{CH}_{3}$

Rxn with: $\mathrm{O}, \mathrm{O}_{2}, \mathrm{O}_{3}, \mathrm{NO}, \mathrm{NO}_{2}, \mathrm{SO}_{2} \ldots$

50. $\mathrm{CH}_{3} \mathrm{O}$

Rxn with: $\mathrm{O}_{2}, \mathrm{NO}, \mathrm{NO}_{2}, \mathrm{CO}$

51. $\mathrm{CH}_{3} \mathrm{O}_{2}$

Rxn with: $\mathrm{O}_{3}, \mathrm{NO}, \mathrm{NO}_{2}, \mathrm{HO}_{2}-\cdots \mathrm{CH}_{3} \mathrm{O}_{2}$

52. $\mathrm{CH}_{3} \mathrm{OH}$

Rxn with: HO - - -

53. $\mathrm{CH}_{3} \mathrm{OOH}$

Rxn with: $\mathrm{H}-{ }_{-}$

54. $\mathrm{CH}_{3} \mathrm{NO}_{2}$ Rxn with: 0 , $\mathrm{HO}-\ldots$

$\mathrm{CH}_{3} \mathrm{ONO}$ Rxn with: $\mathrm{O}, \mathrm{O}_{3}, \mathrm{H}, \mathrm{HO}-\cdots$ hv

55. $\mathrm{CH}_{3} \mathrm{NO}_{3}$ Rxn with:

56. $\mathrm{CH}_{4}$ Rxn with: $O, O\left({ }^{1} \mathrm{D}\right), \mathrm{O}\left({ }^{1} \mathrm{~S}\right), \mathrm{O}_{3}, \mathrm{HO}, \mathrm{Cl}, \mathrm{ClO} \ldots$

$56 \mathrm{a} \cdot \mathrm{C}_{2} \mathrm{H}_{2}$ Rxn with: HO, ClO - -

57. $\mathrm{C}_{2} \mathrm{H}_{4}$ Rxn with: $\mathrm{O}, \mathrm{O}_{3}, \mathrm{HO}, \mathrm{HO}_{2}, \mathrm{ClO} \ldots$ 
58. $\mathrm{C}_{2}{ }^{\mathrm{H}} 6 \quad$ Rxn with: $\mathrm{O}, \mathrm{O}\left({ }^{1} \mathrm{D}\right), \mathrm{HO}, \mathrm{HO}_{2}, \mathrm{C} 1-$

*59. $\mathrm{C}_{3} \mathrm{H}_{6} \quad$ Rxn with: $0, \mathrm{O}_{3}, \mathrm{HO}-\ldots$

+60. $\mathrm{C}_{3} \mathrm{H}_{8} \quad$ Rxn with: $\mathrm{O}, \mathrm{HO}, \mathrm{HO}_{2}-\cdots$

$\nabla 60 a$. ${ }_{6}{ }_{6}{ }_{6} \quad$ Rxn with: 0 , HO - - -

61. $\mathrm{CF}_{2} \mathrm{Cl}_{2}$ Rxn with: O $\left({ }^{1} \mathrm{D}\right), \mathrm{O}_{2}\left({ }^{1} \Delta\right)$, HO - - hv

62. $\mathrm{CFCl}_{3}$ Rxn with: $O\left({ }^{1} \mathrm{D}\right)$, HO.$- \mathrm{h} \nu$

63. $\mathrm{CCl}_{4}$ Rxn with: HO - - hv

64. $\mathrm{CH}_{i}{ }^{\mathrm{Cl}} j \mathrm{Rxn}$ with: $\mathrm{O}, \mathrm{O}_{2}\left({ }^{1} \Delta\right), \mathrm{O}_{3}, \mathrm{H}, \mathrm{HO}-{ }_{-}$

165. $\mathrm{CH}_{i} \mathrm{Cl}_{j} \mathrm{~F}_{\mathrm{k}}$ Rxn with: HO - -

* and higher alkenes

$\dagger$ and higher alkanes

$\nabla$ and other aromatics

$\checkmark$ and other halocarbons 


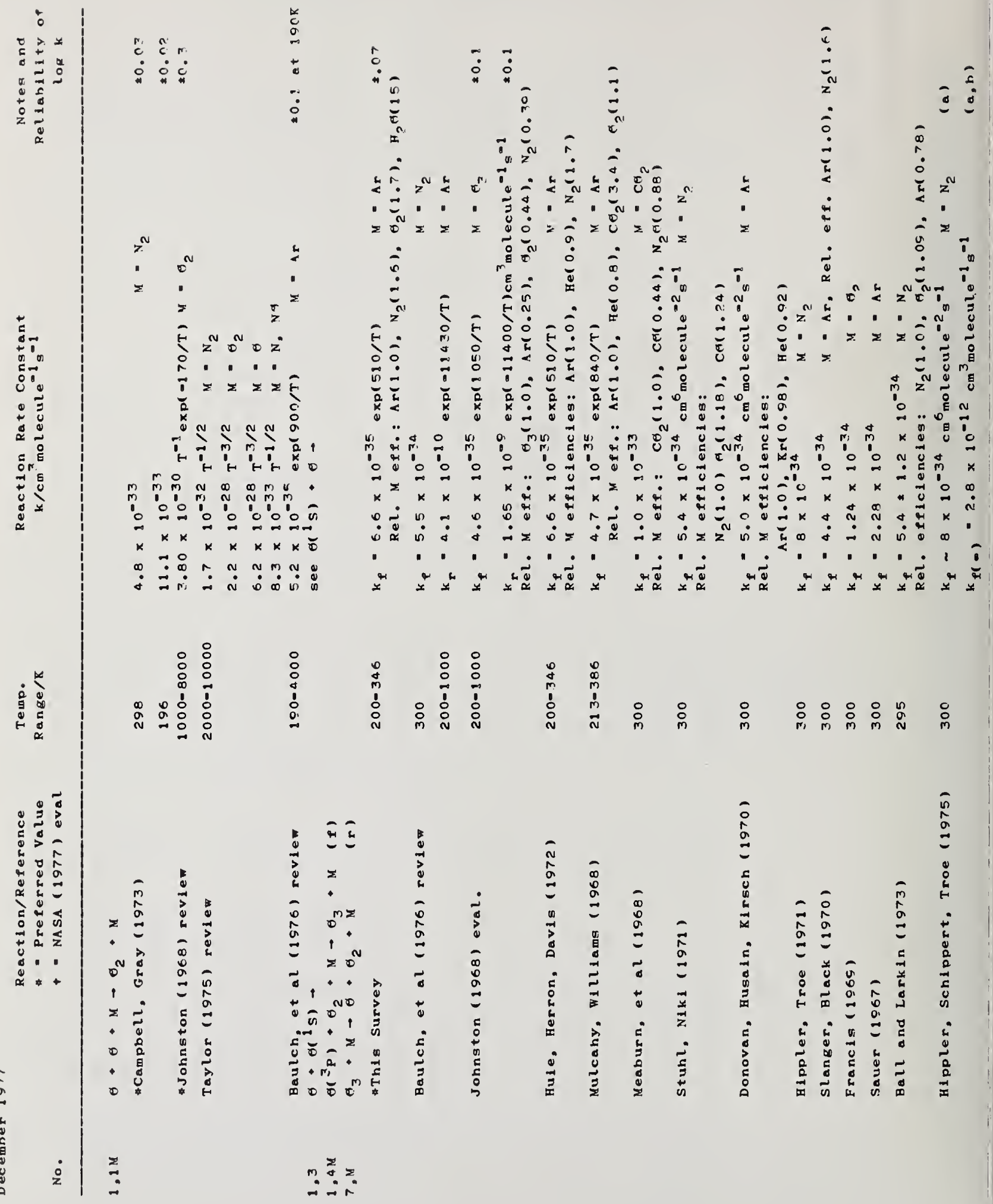




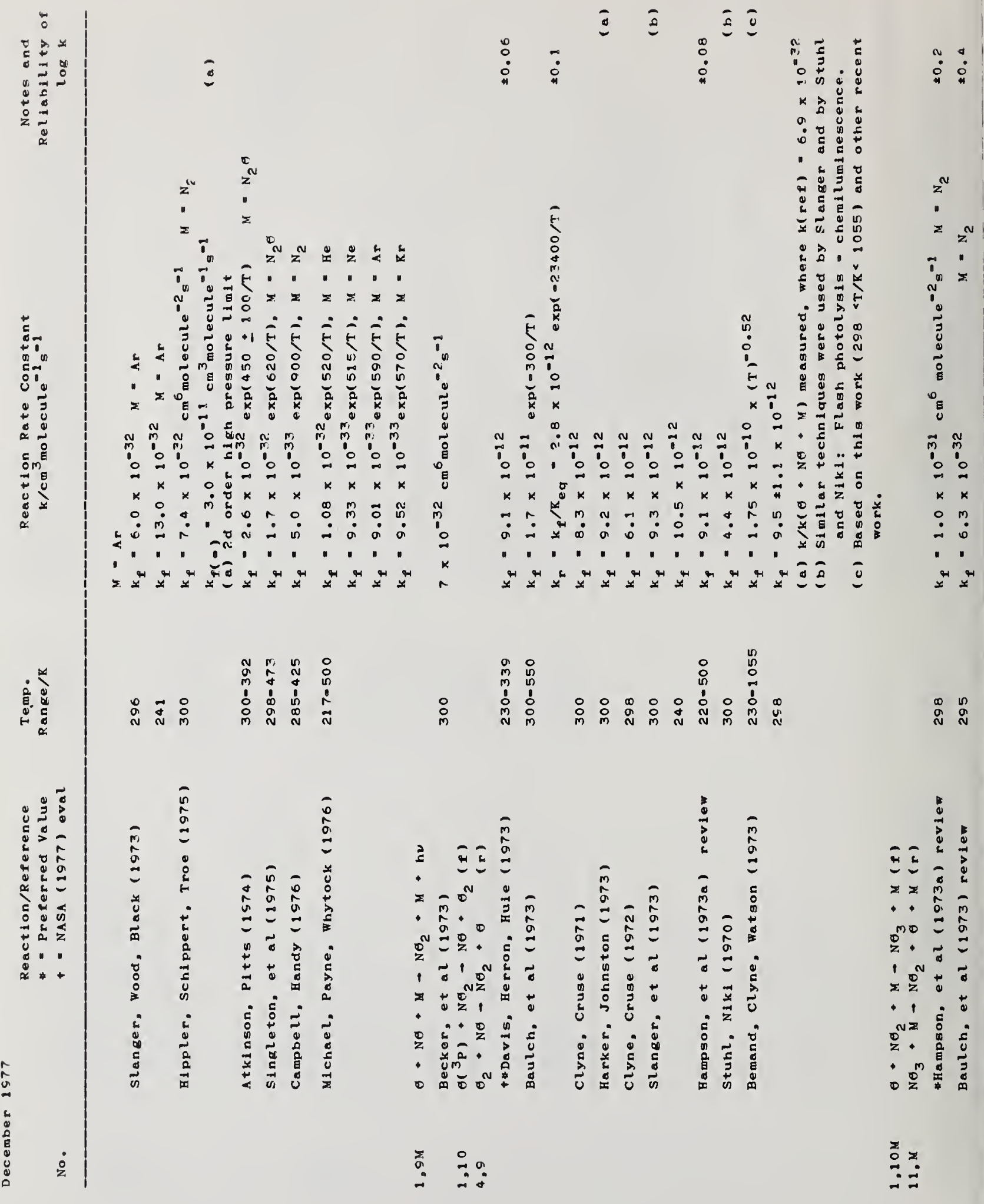




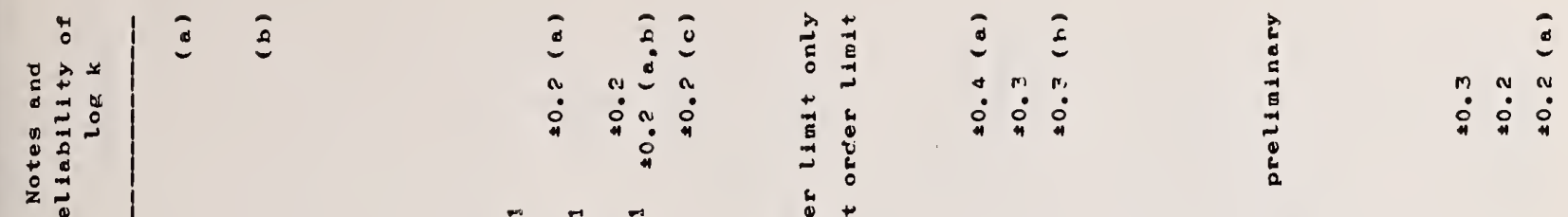

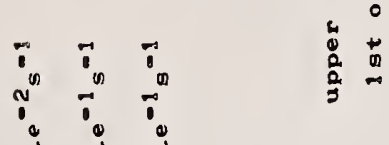

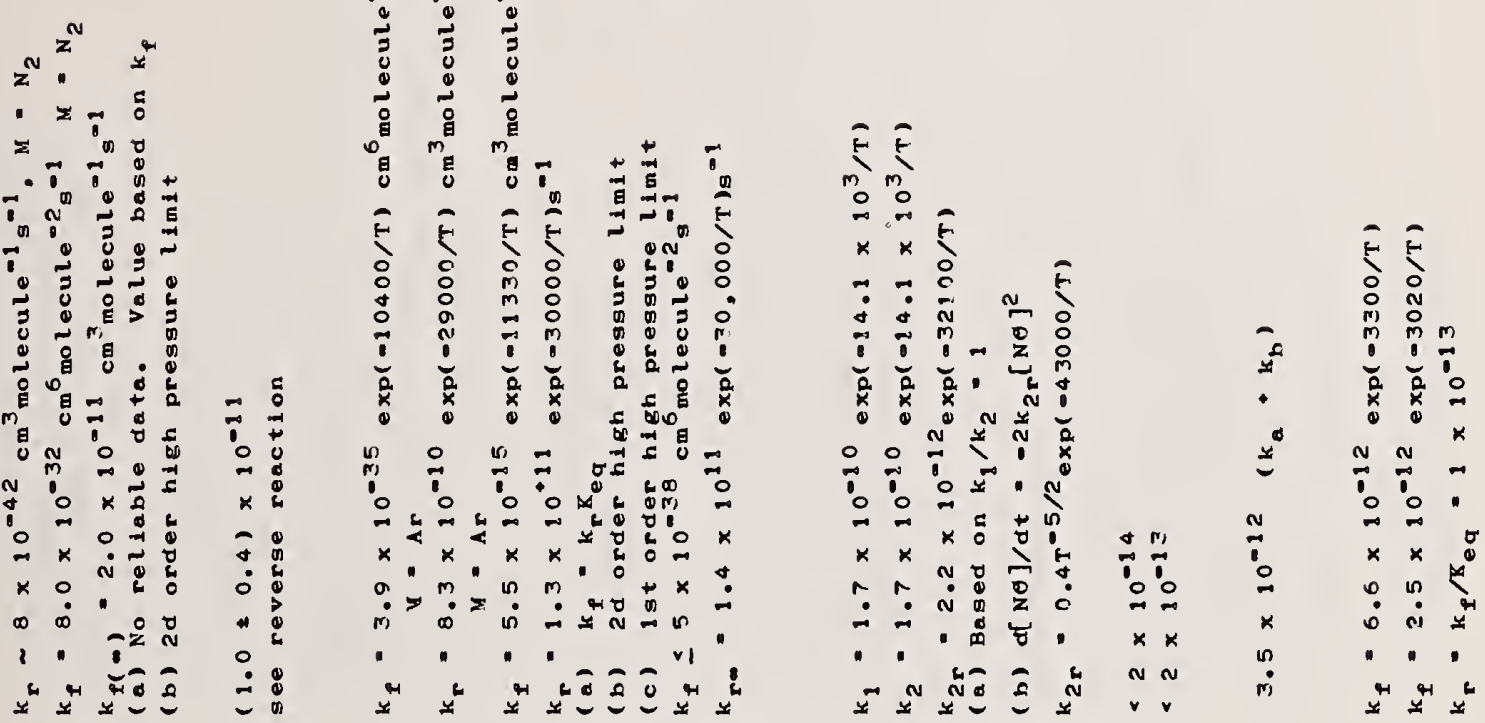

$$
\begin{aligned}
& \text { : }
\end{aligned}
$$

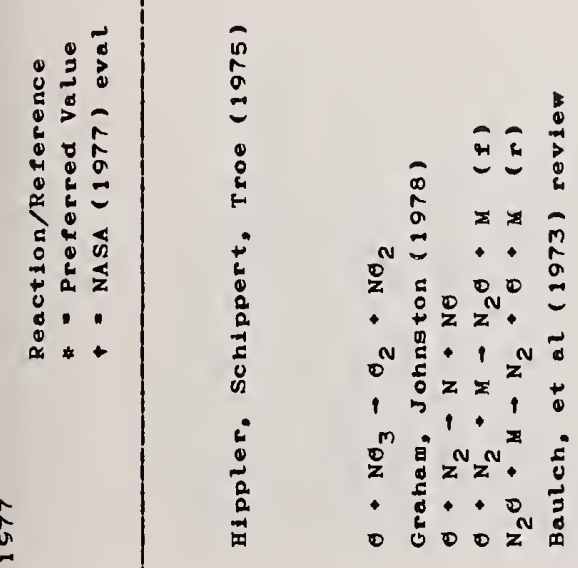

$$
\begin{aligned}
& \dot{\vdots} \equiv \\
& \stackrel{m}{m} \underset{\sim}{m}:
\end{aligned}
$$

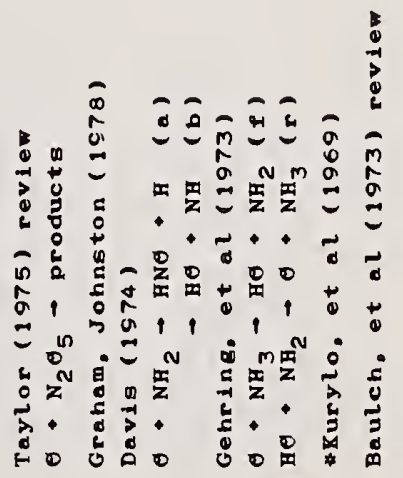

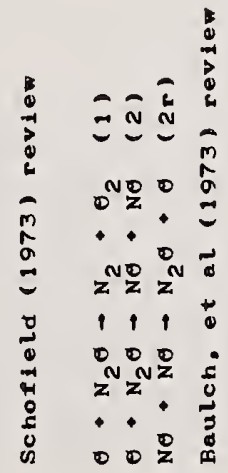

$$
\begin{aligned}
& \because \quad \because \quad \because
\end{aligned}
$$




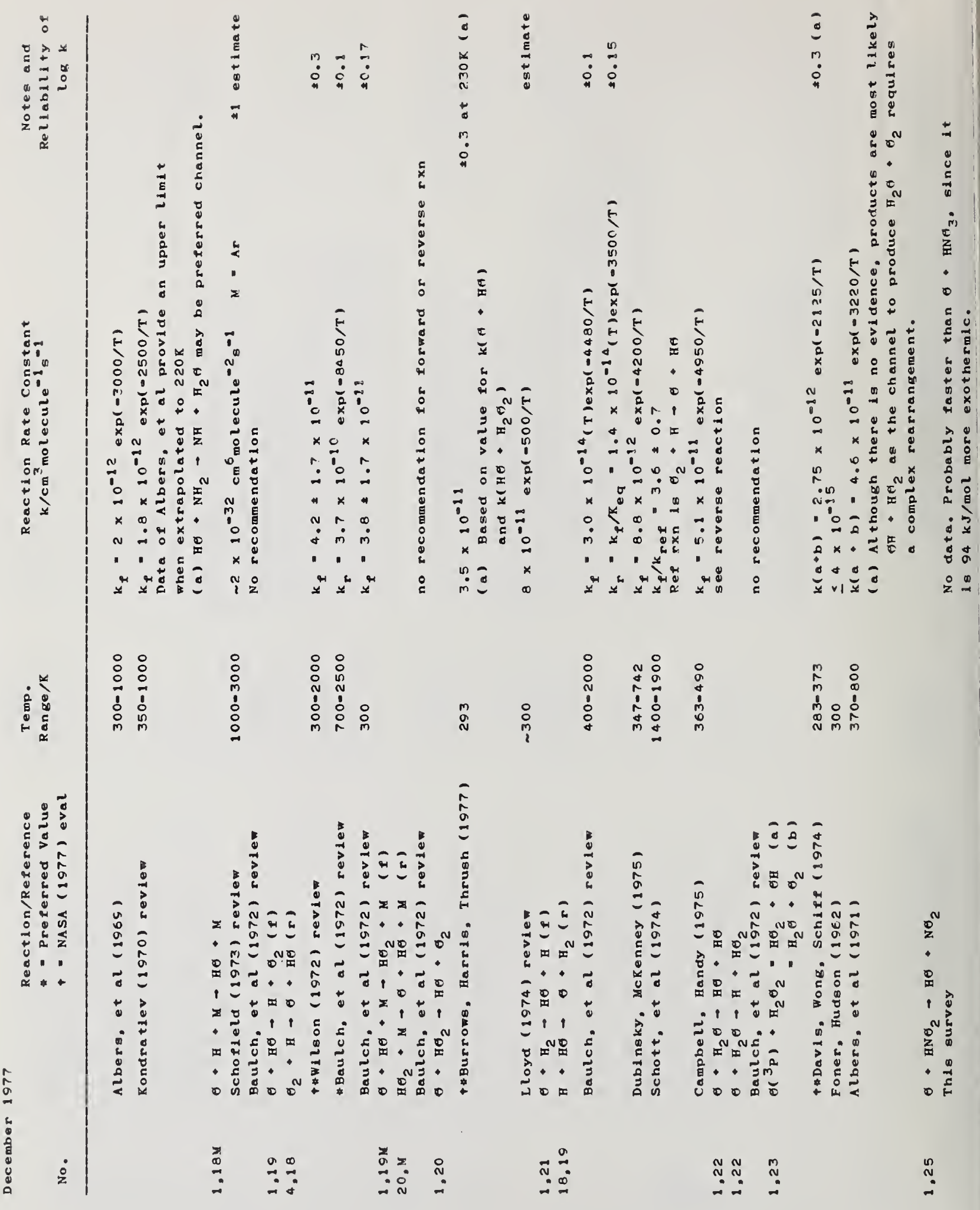




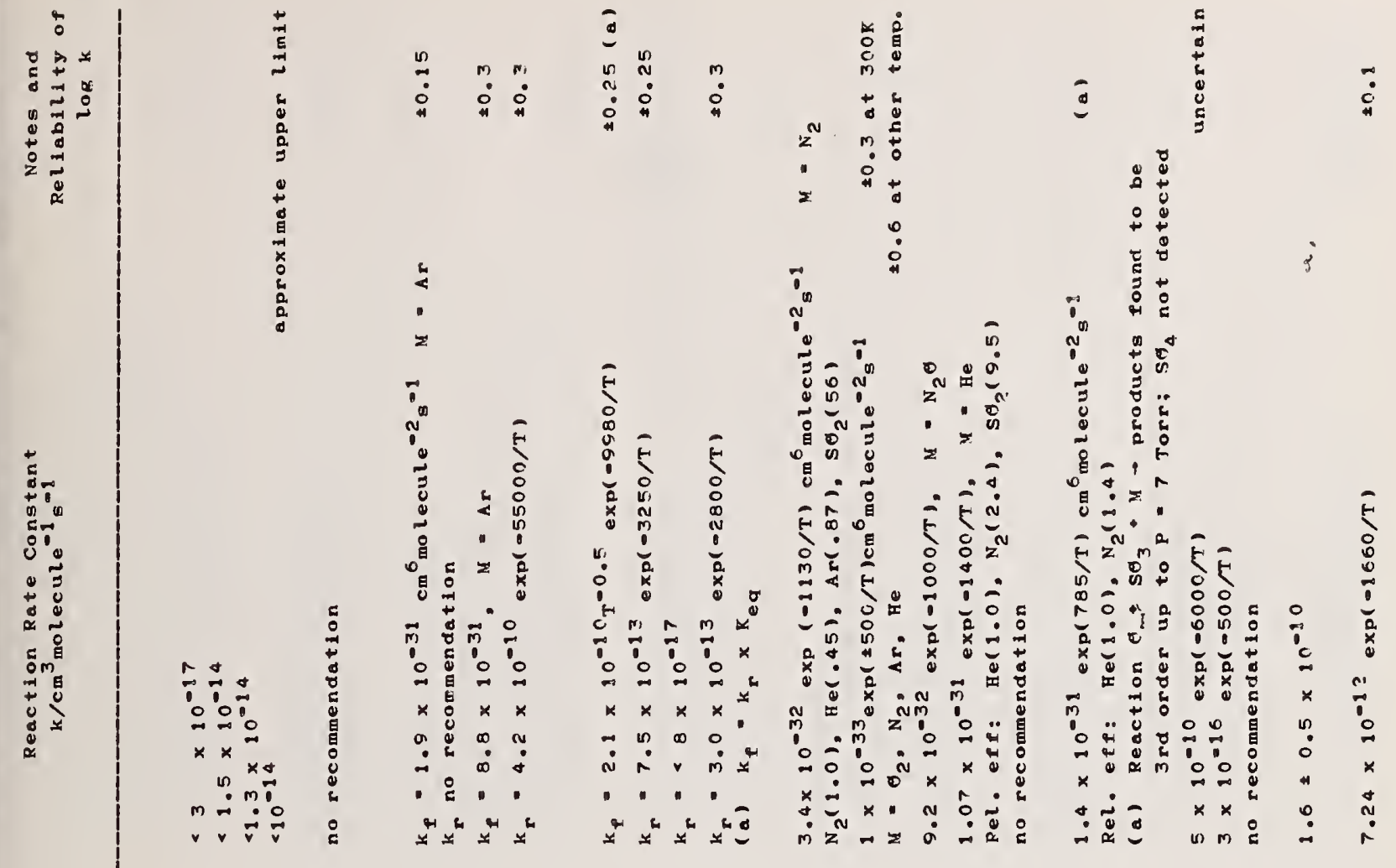

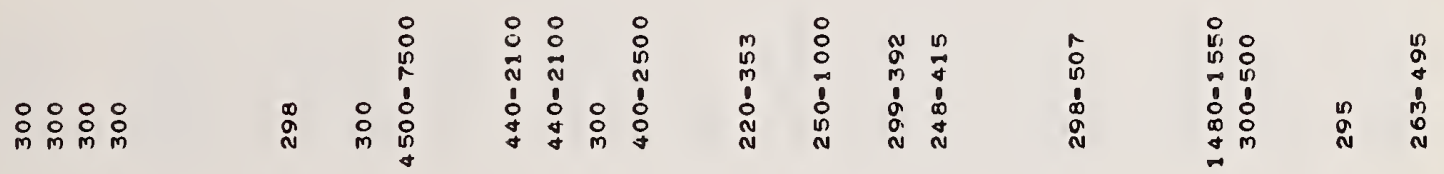

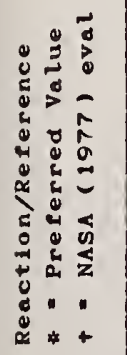

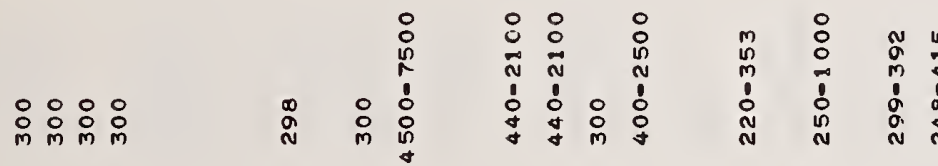

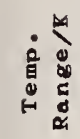

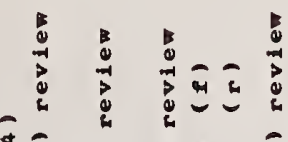

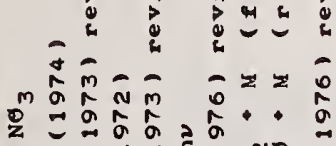

-

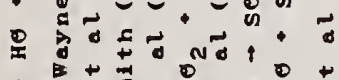

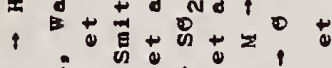

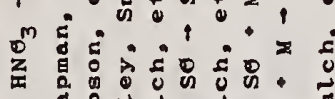

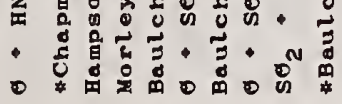

: $\stackrel{\circ}{*}$

$\stackrel{\infty}{N} \hat{N}$

$\stackrel{\substack{2 \\ \infty \\ N}}{\sim}$

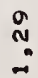

$\stackrel{n}{m} \stackrel{n}{m}:-$ 


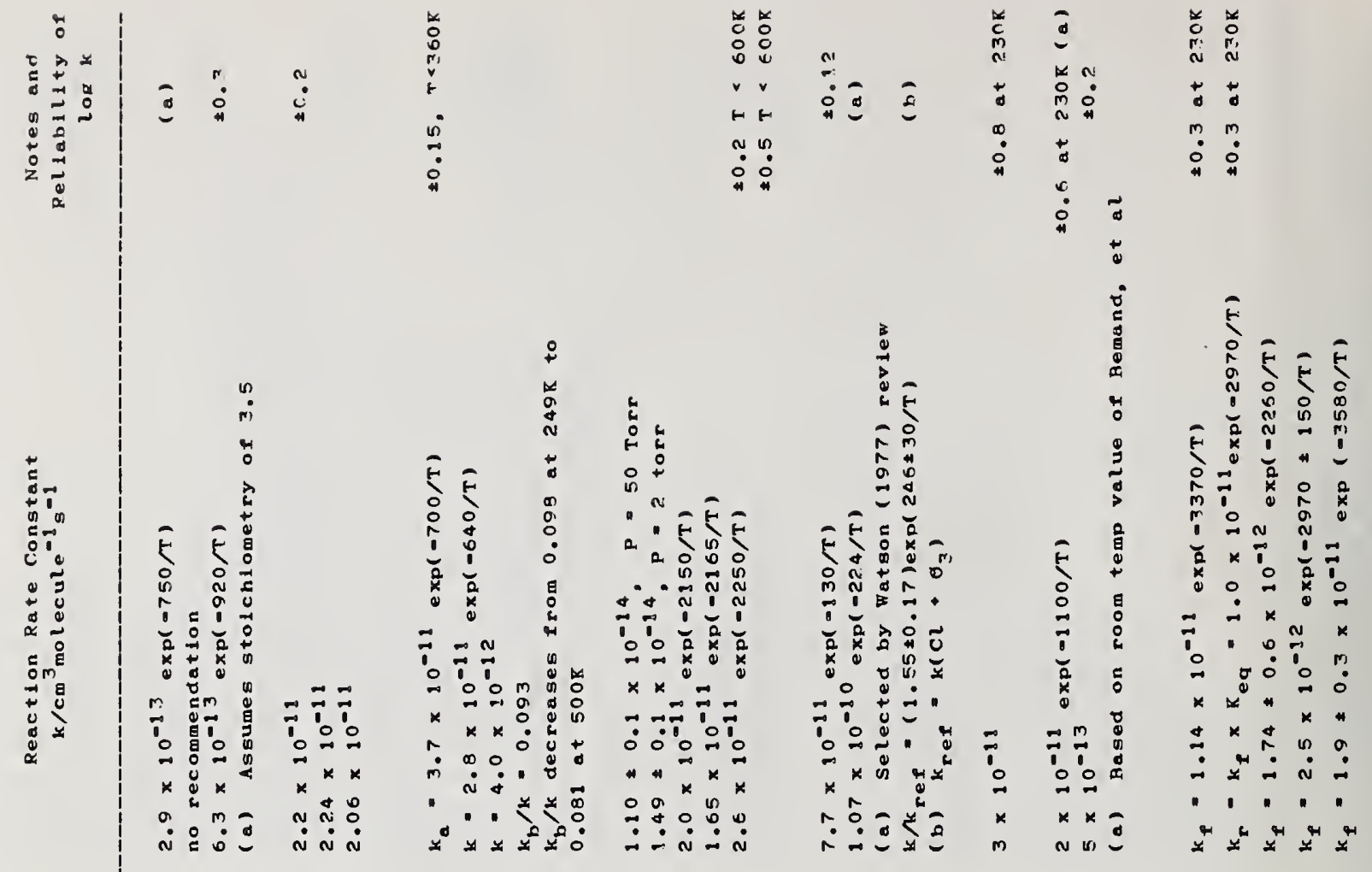

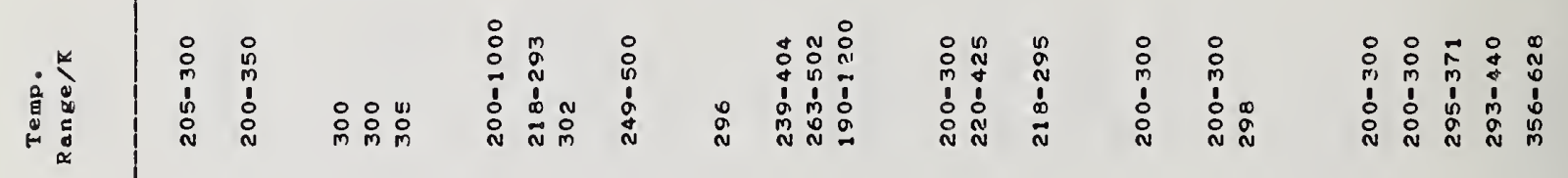

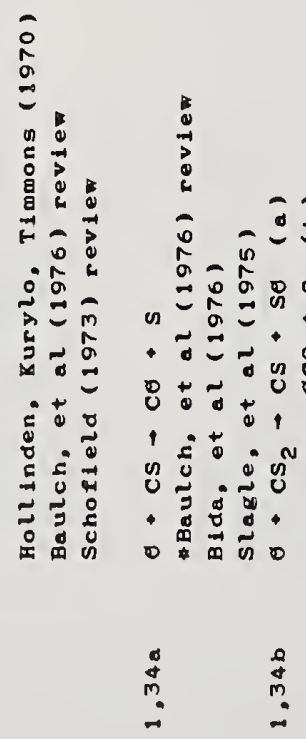



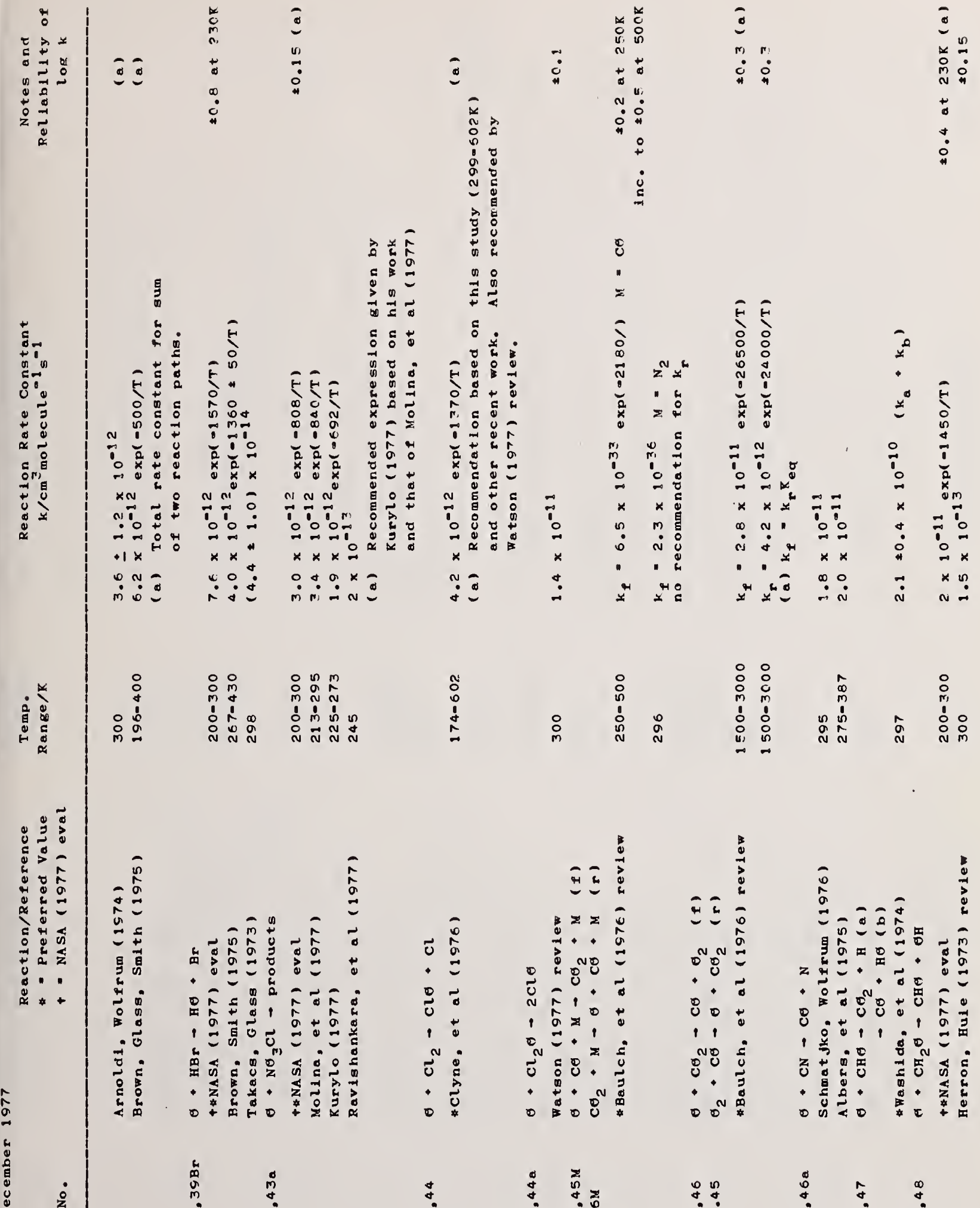

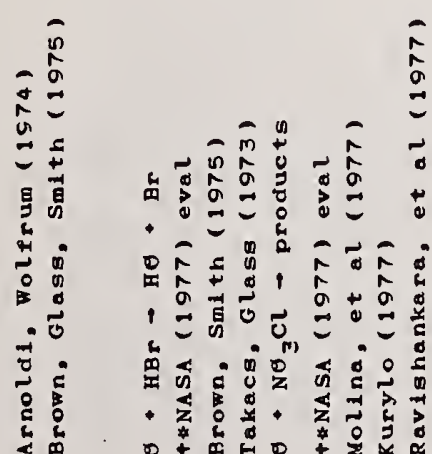

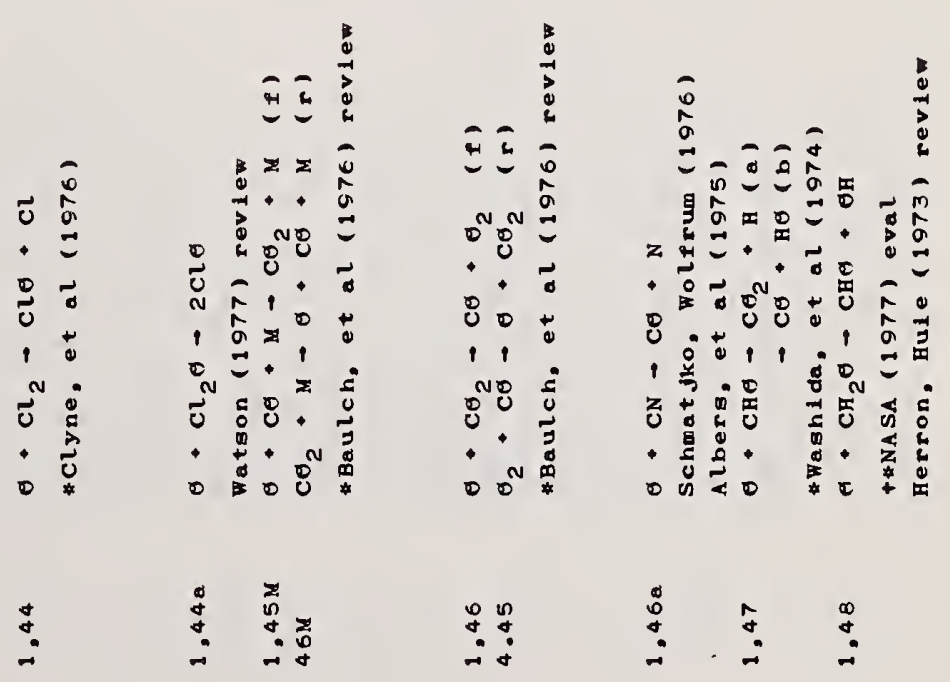




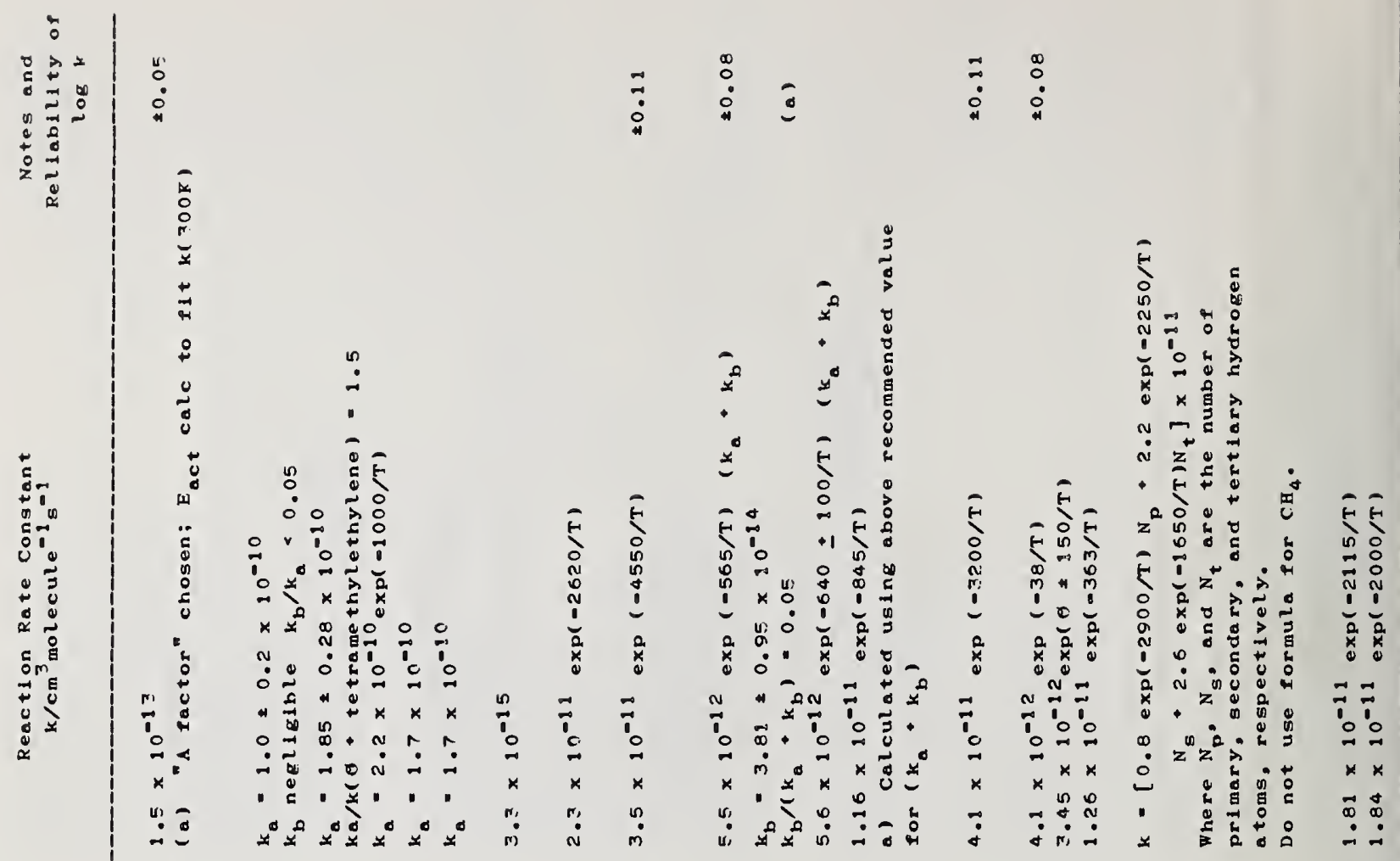

แั
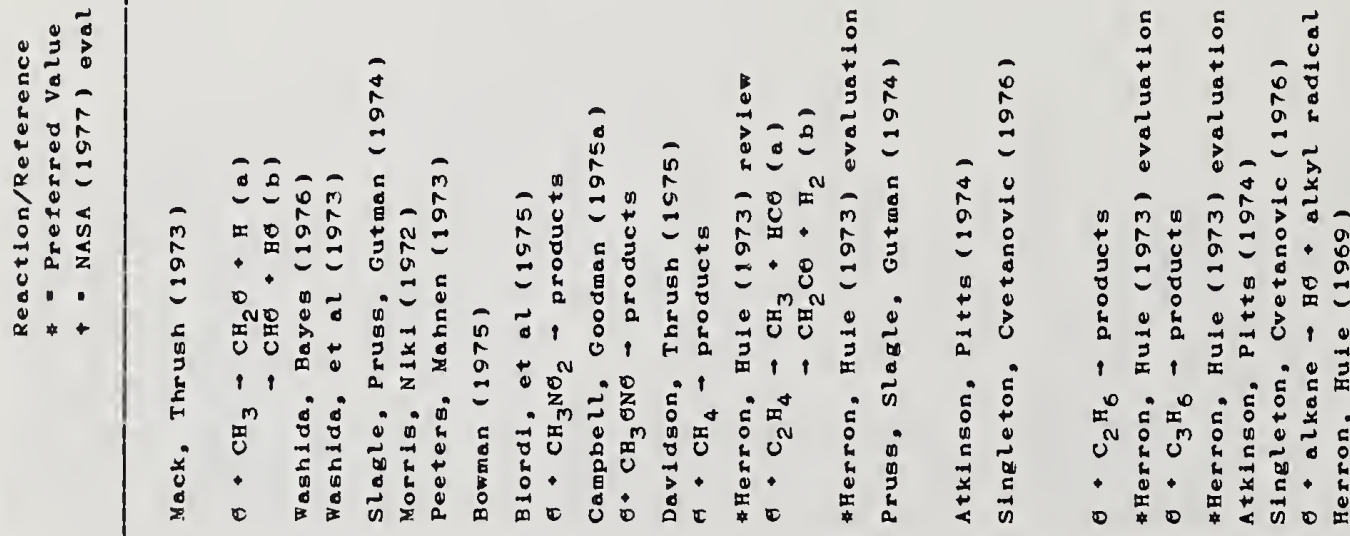

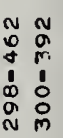

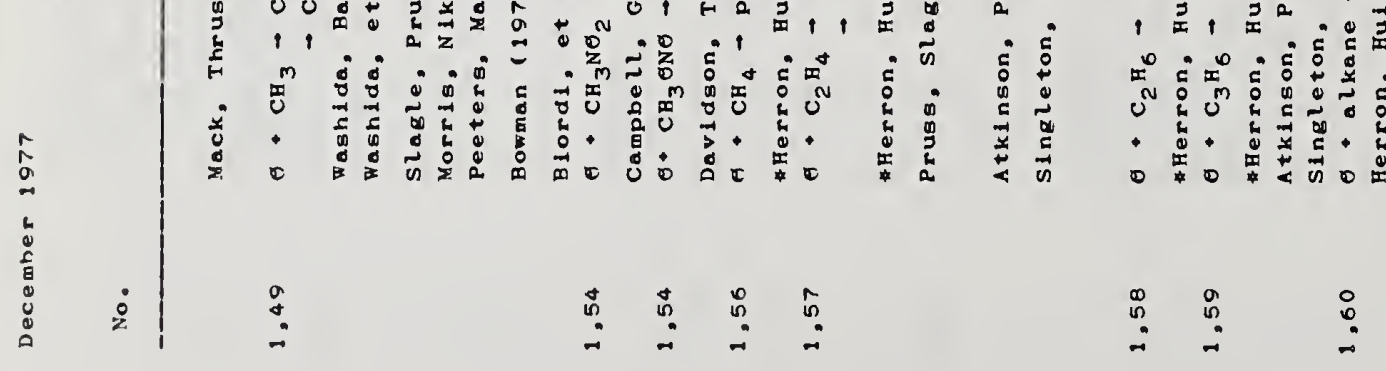




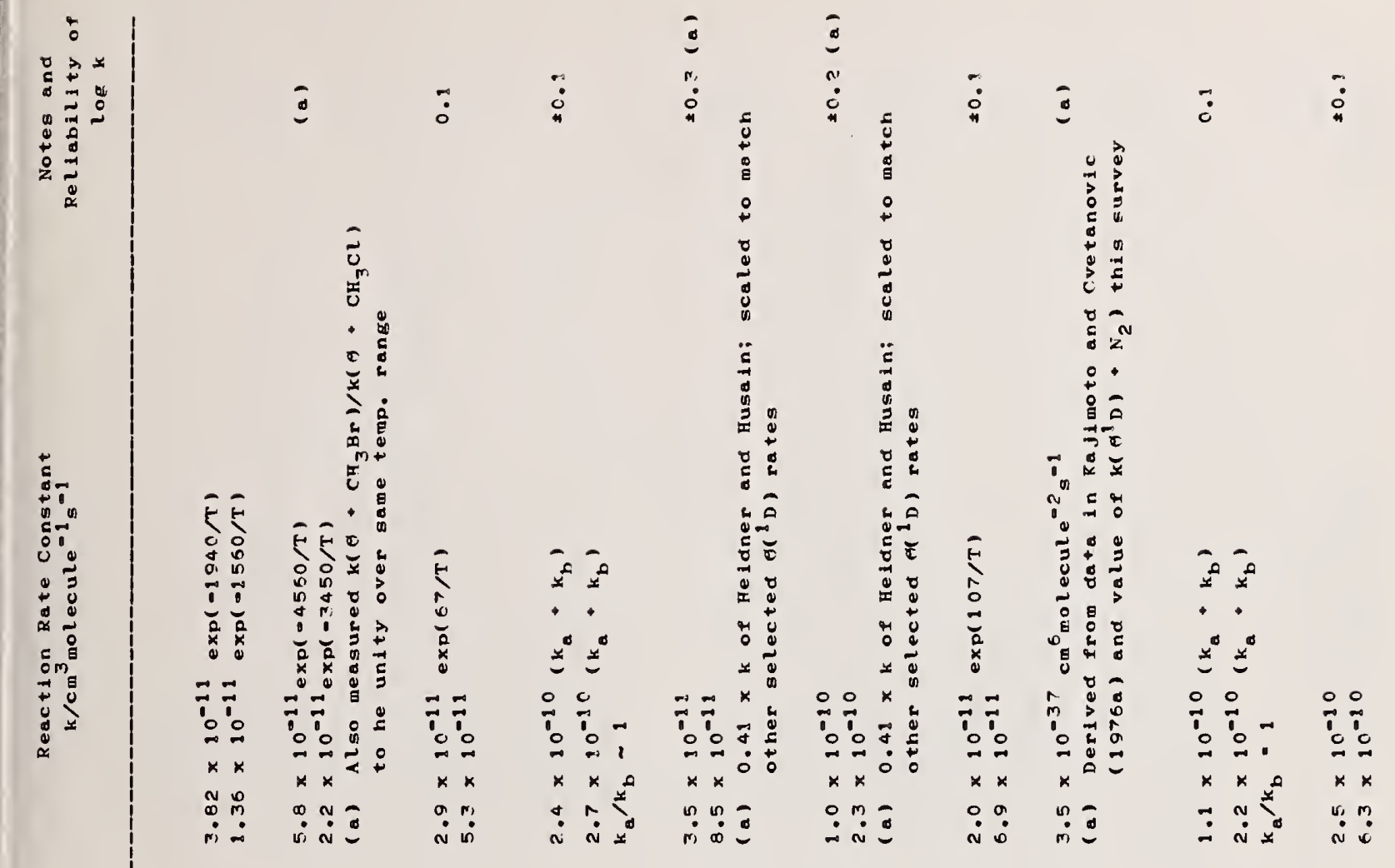

这

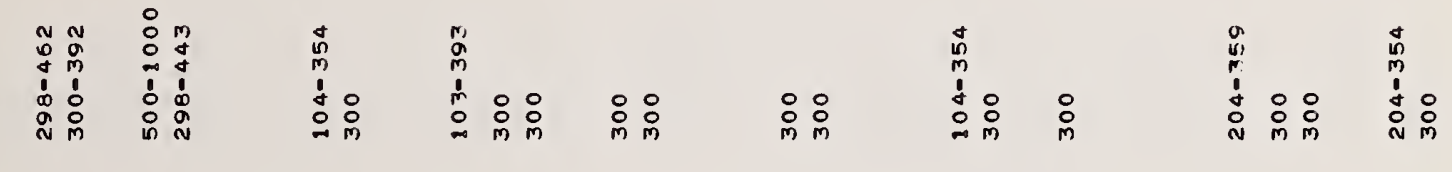

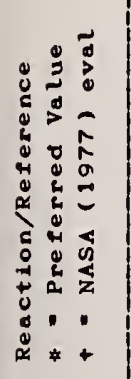

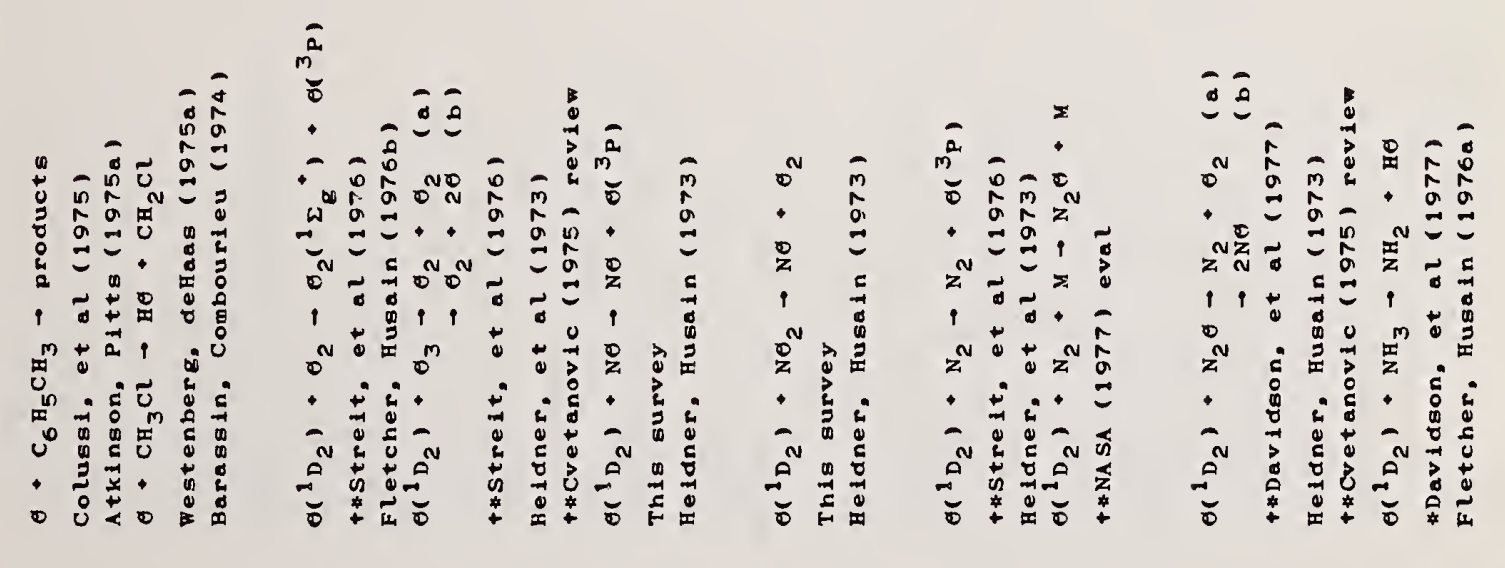

$\dot{0}$

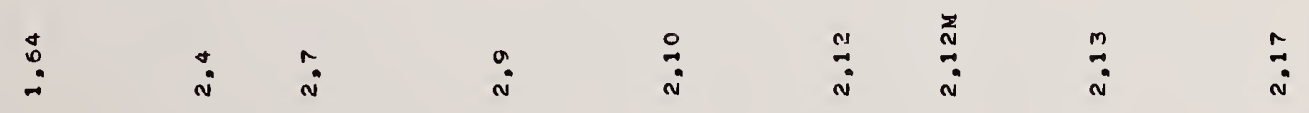




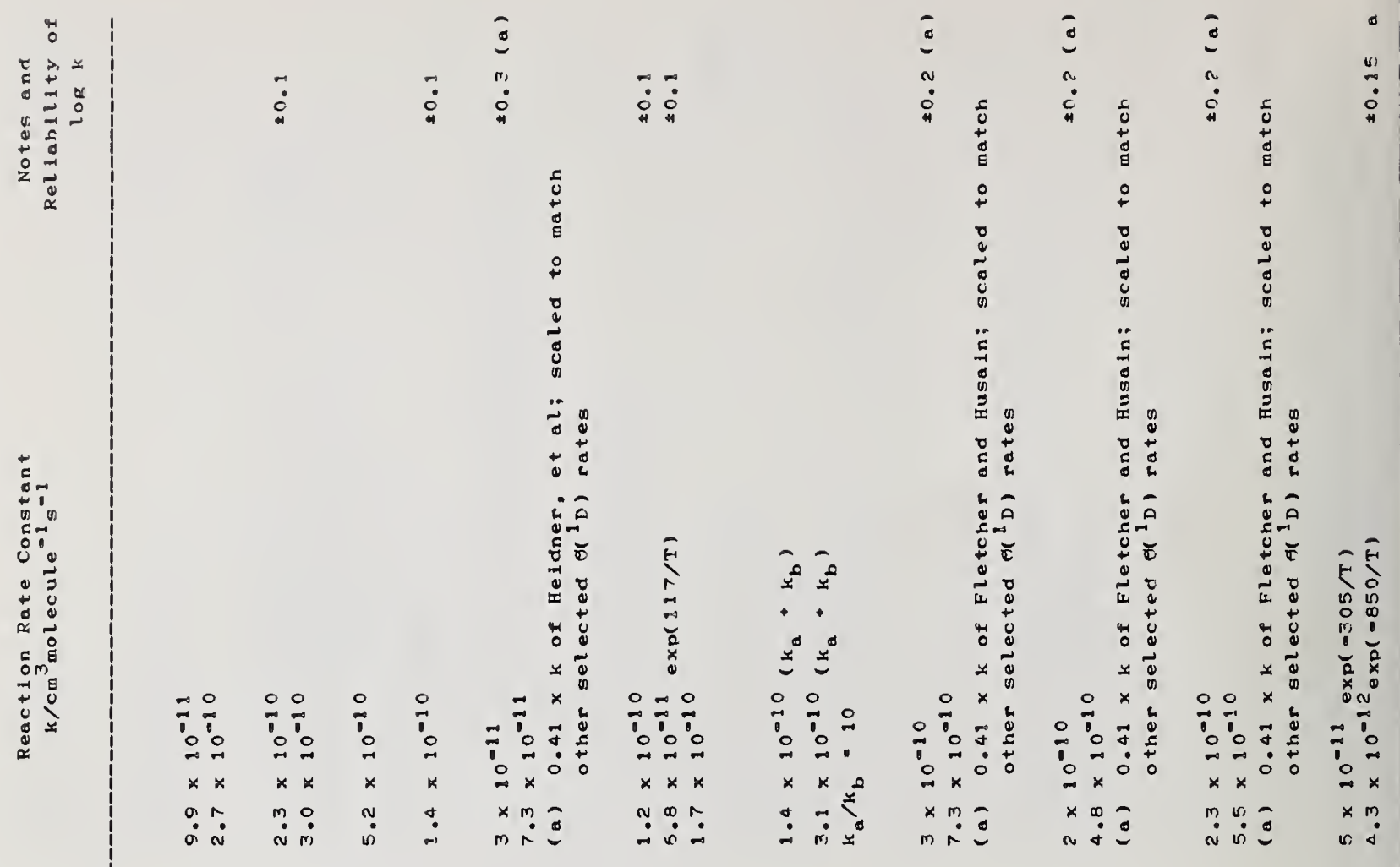

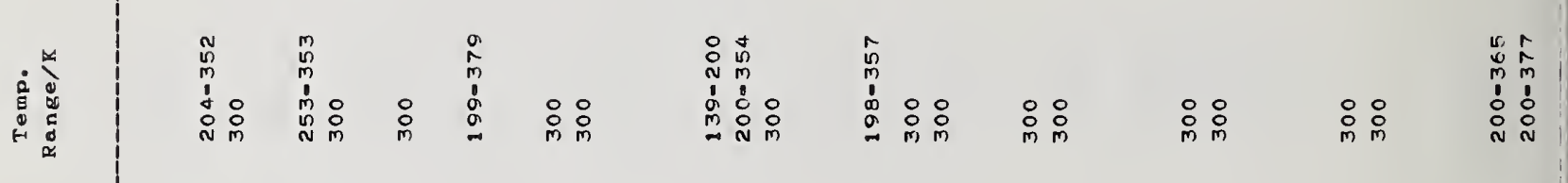

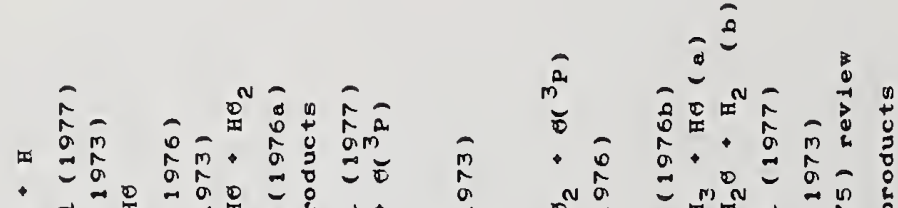

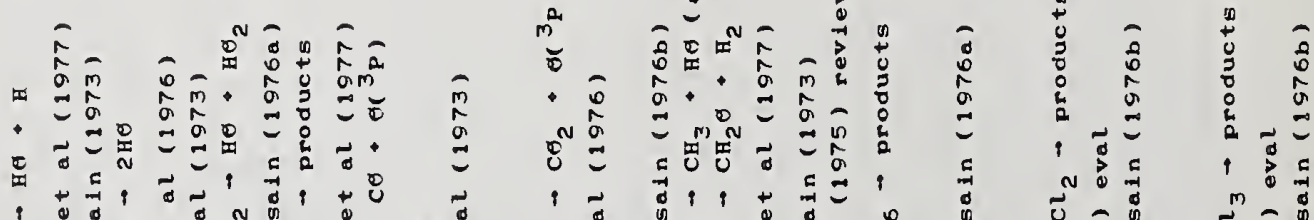

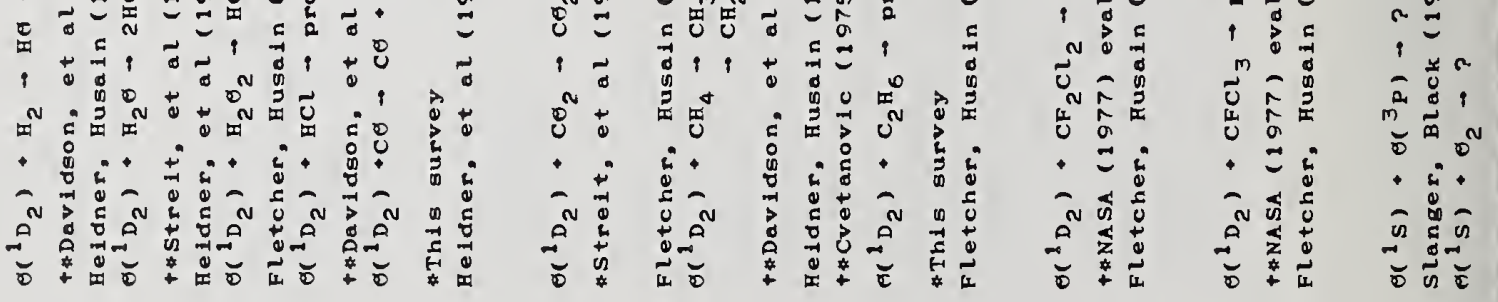

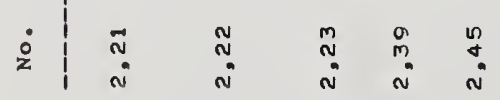

$\stackrel{0}{i} \quad$ in

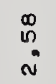

$\overrightarrow{0}$

ڤี

$\because \quad:$ 


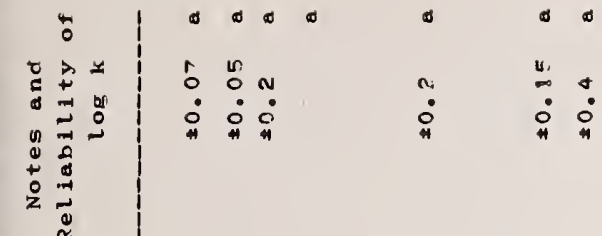

$$
\begin{aligned}
& \stackrel{\sim}{\circ}:
\end{aligned}
$$

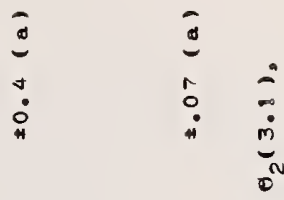

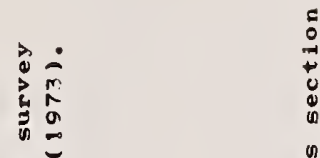

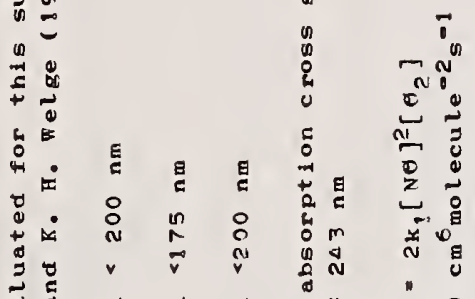

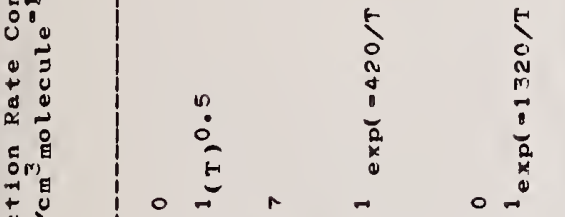

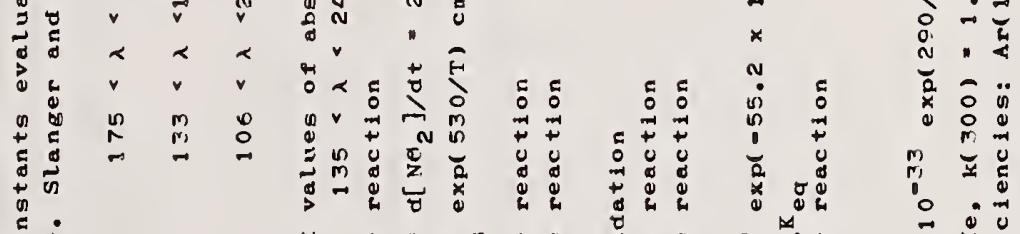

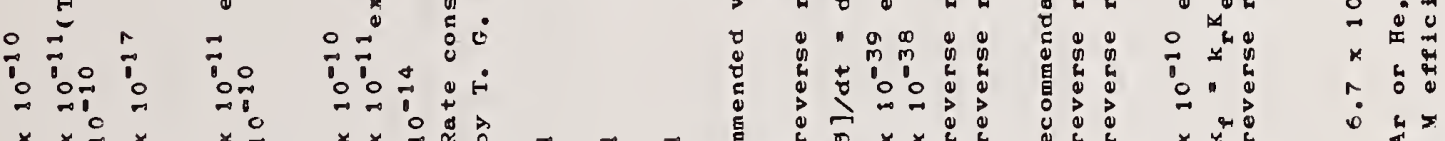

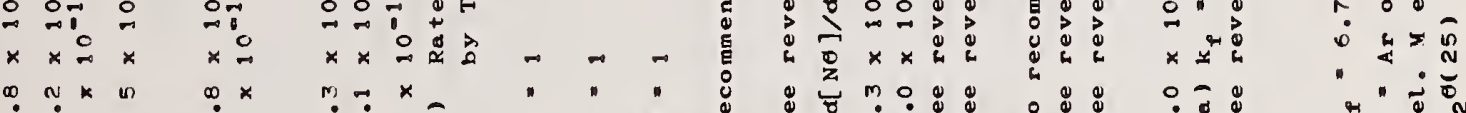

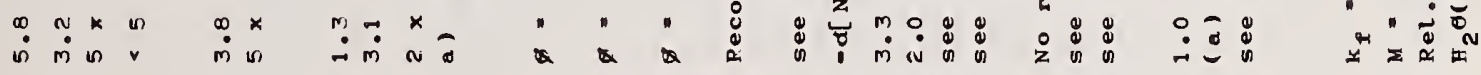

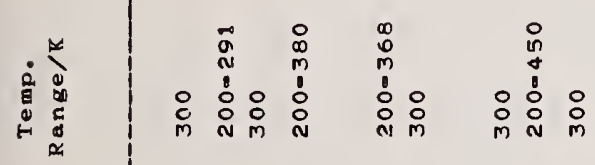

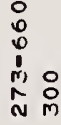

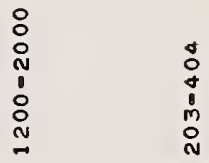

$$
\begin{aligned}
& \text { (2) }
\end{aligned}
$$

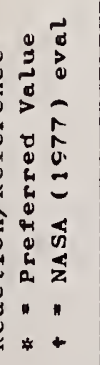

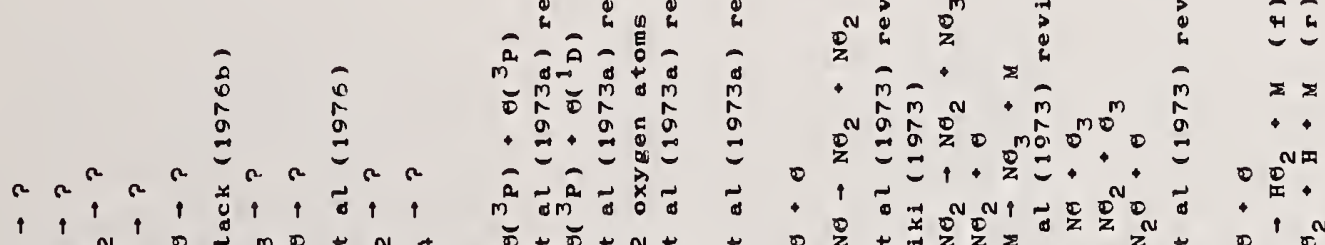

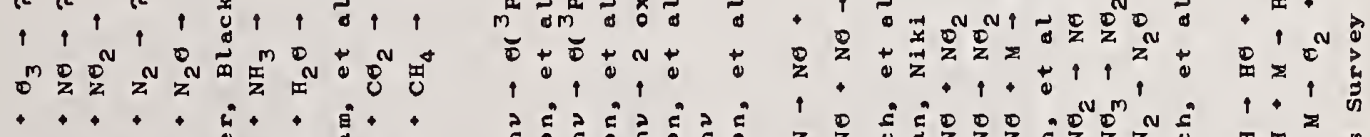

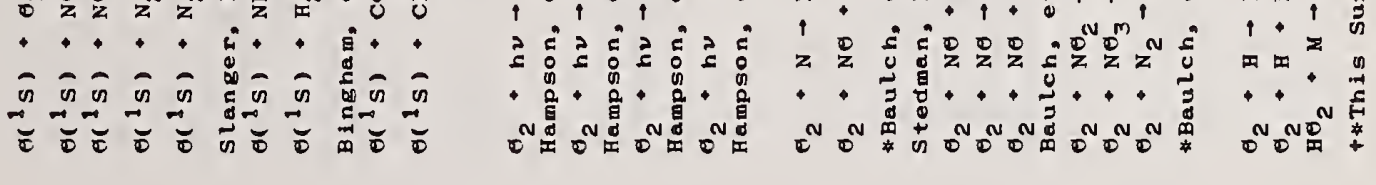

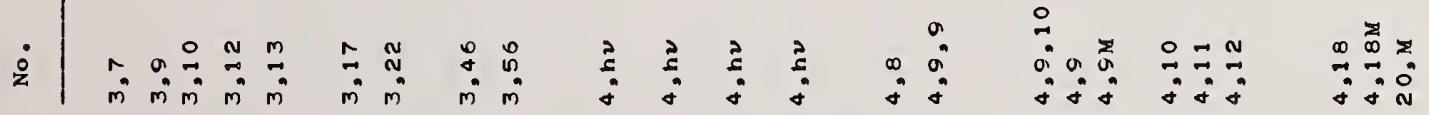




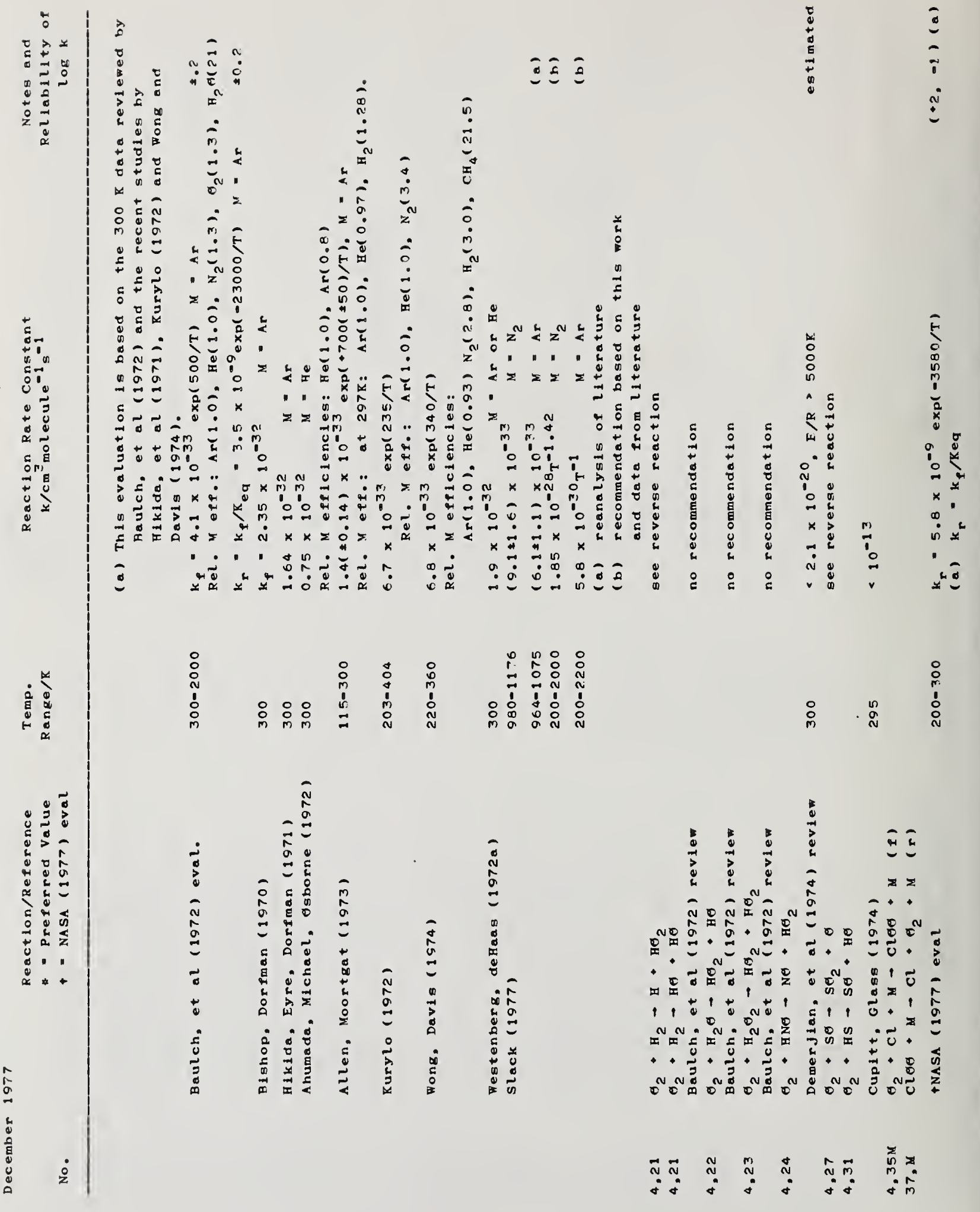




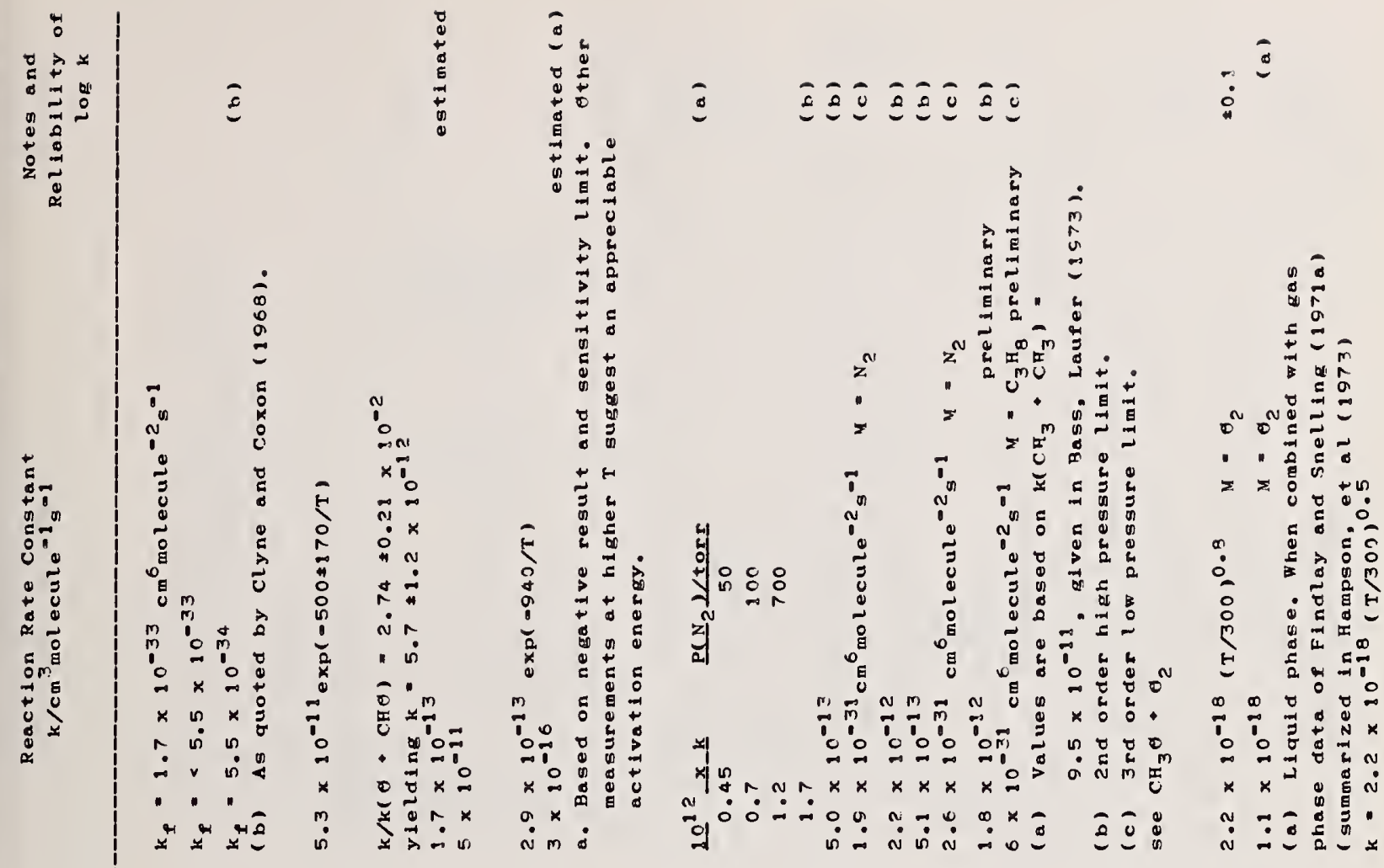

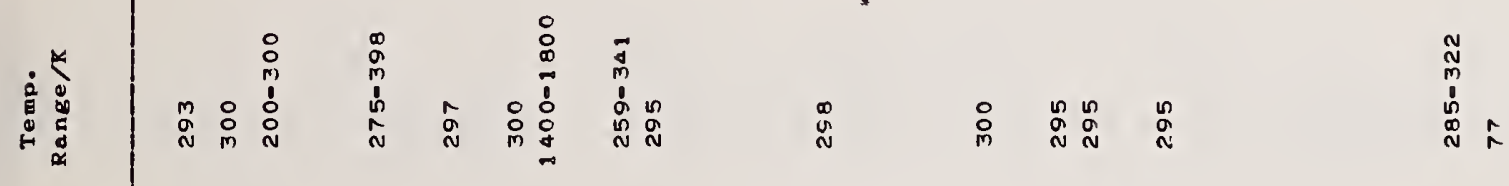

a

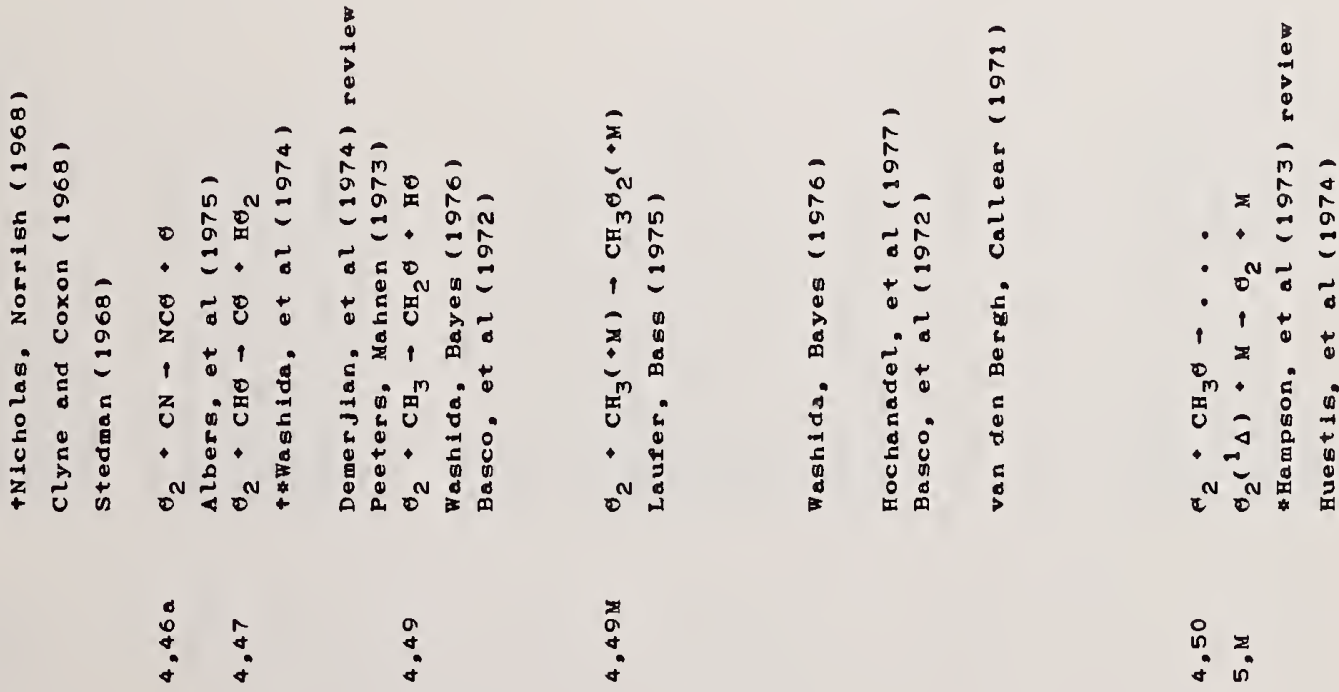




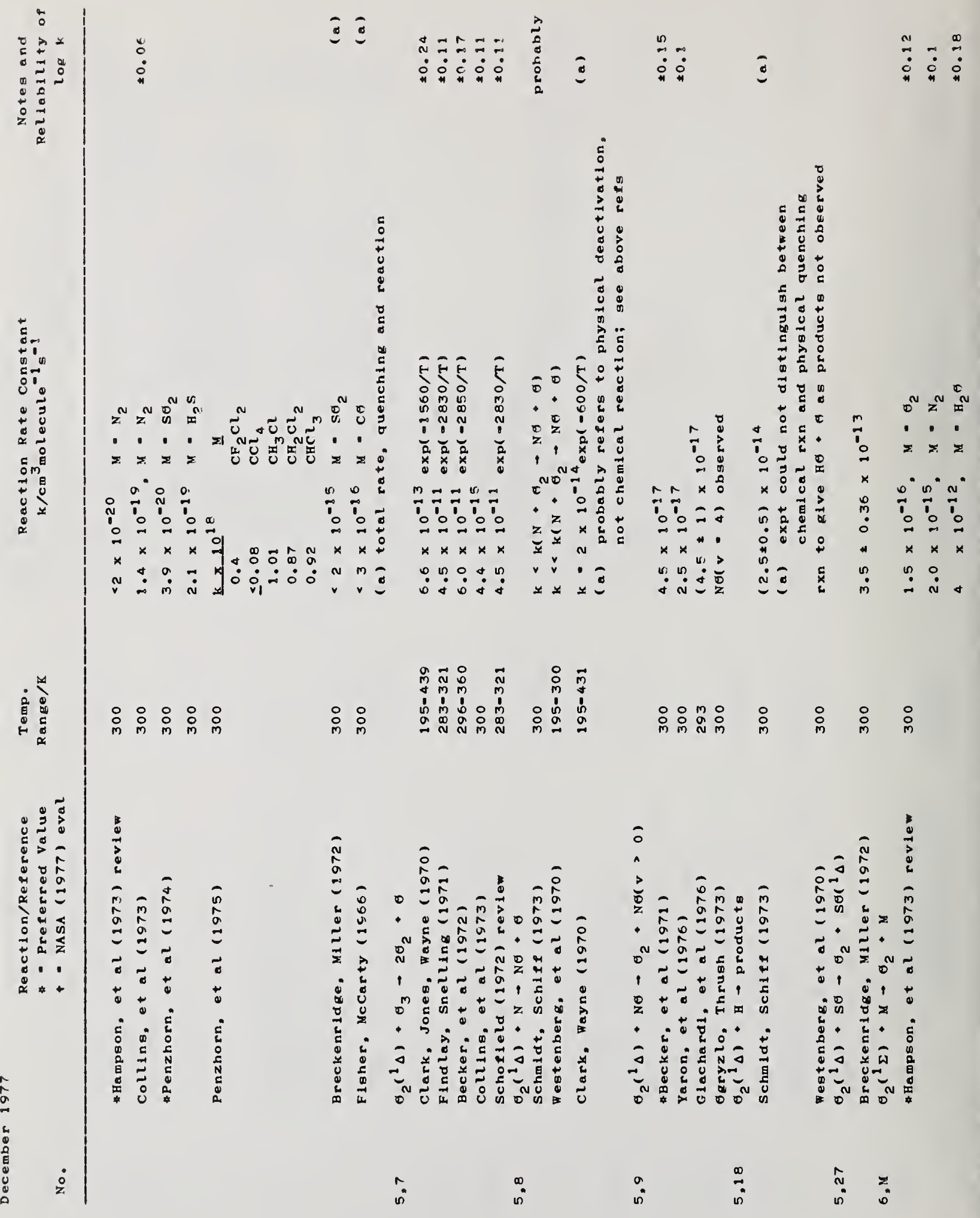




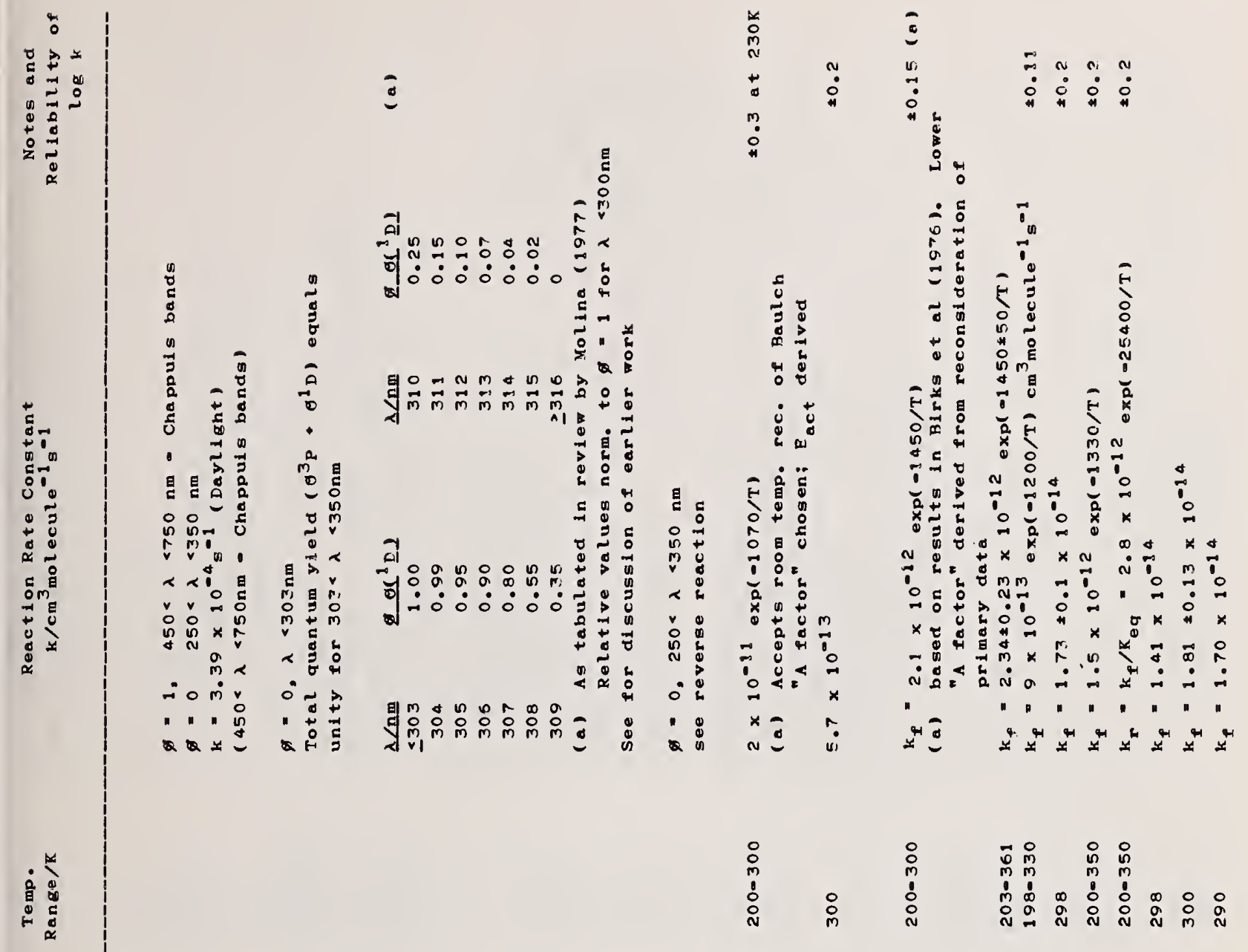

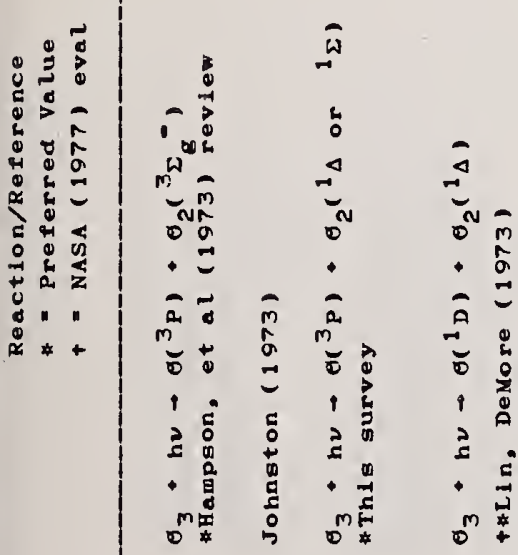

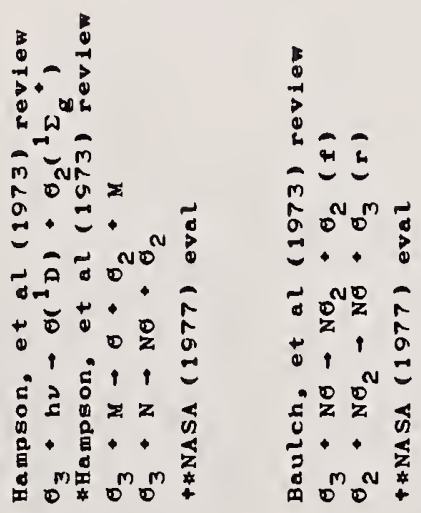

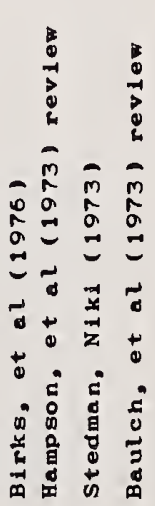

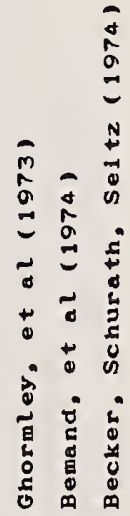

:

$\stackrel{2}{a} \stackrel{0}{i} \stackrel{0}{\circ}$ 


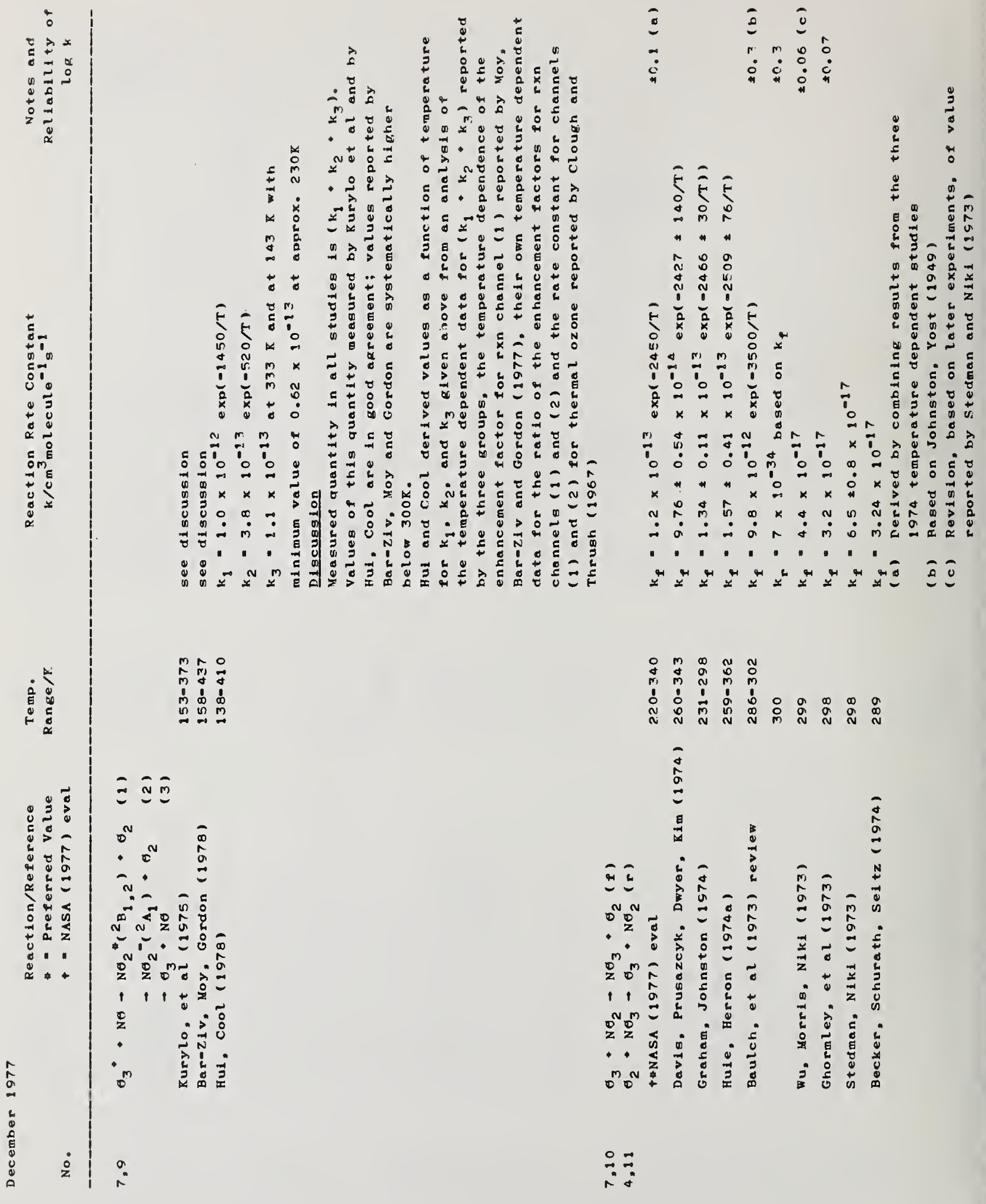




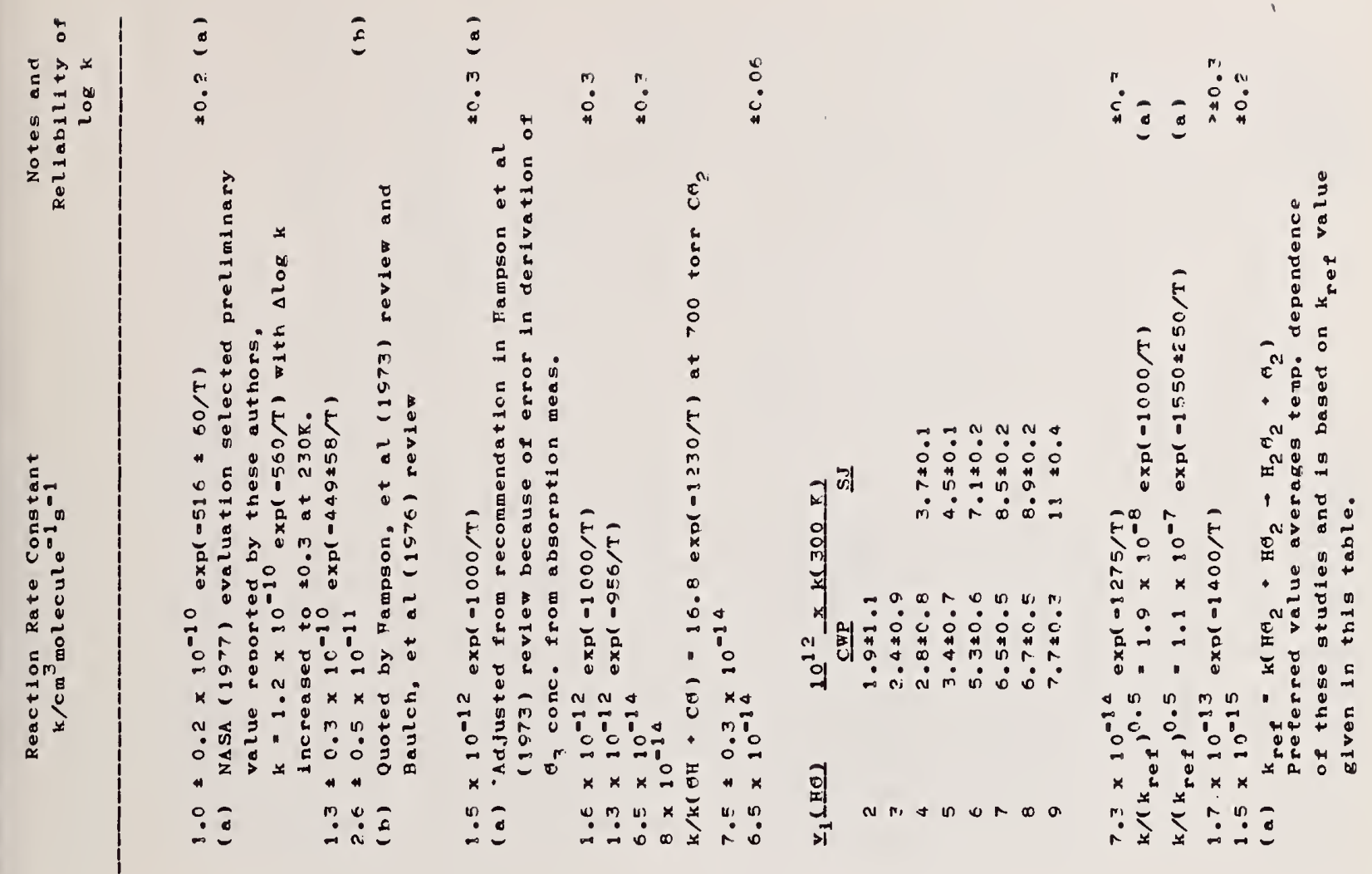

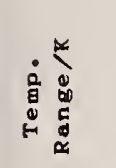
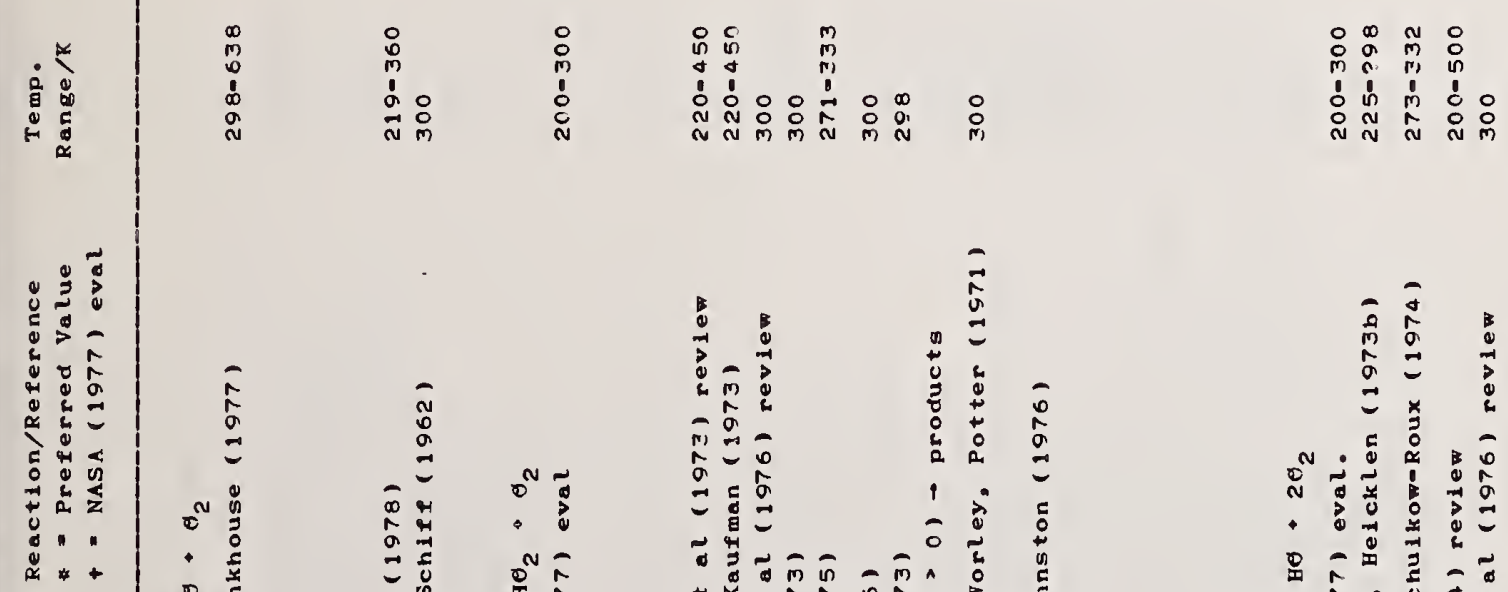

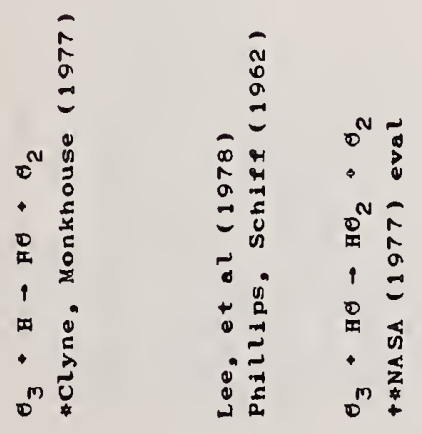

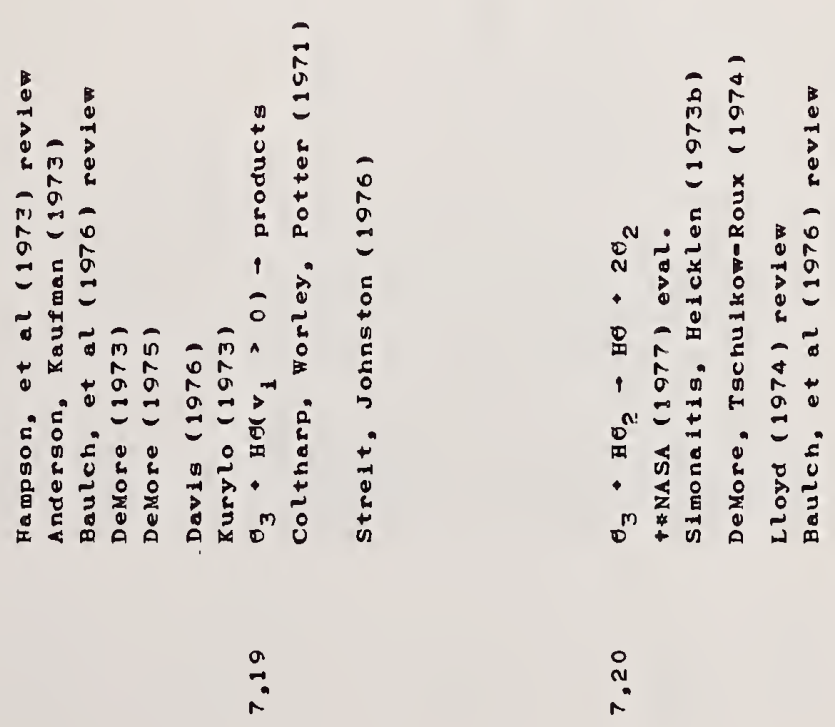




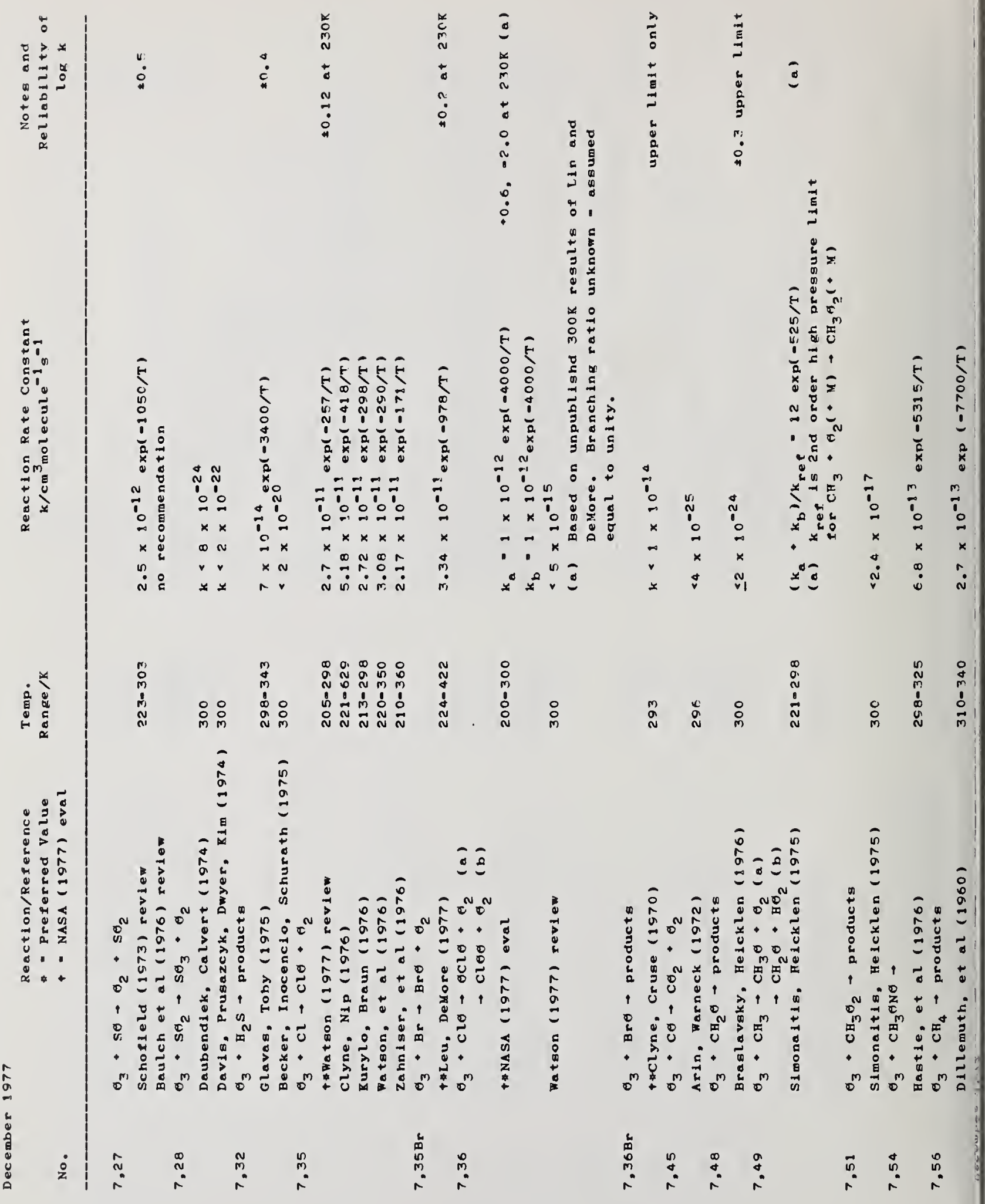




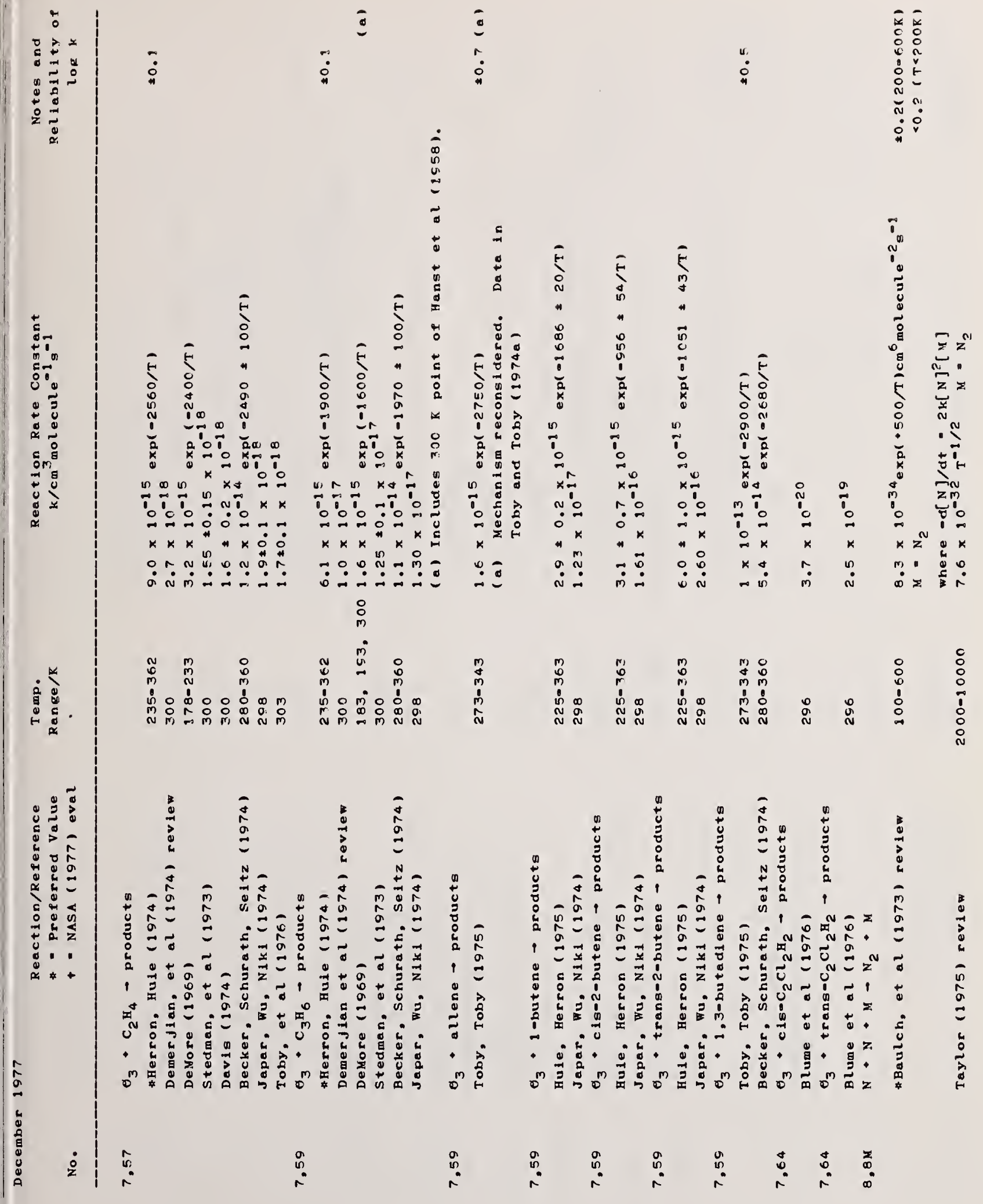




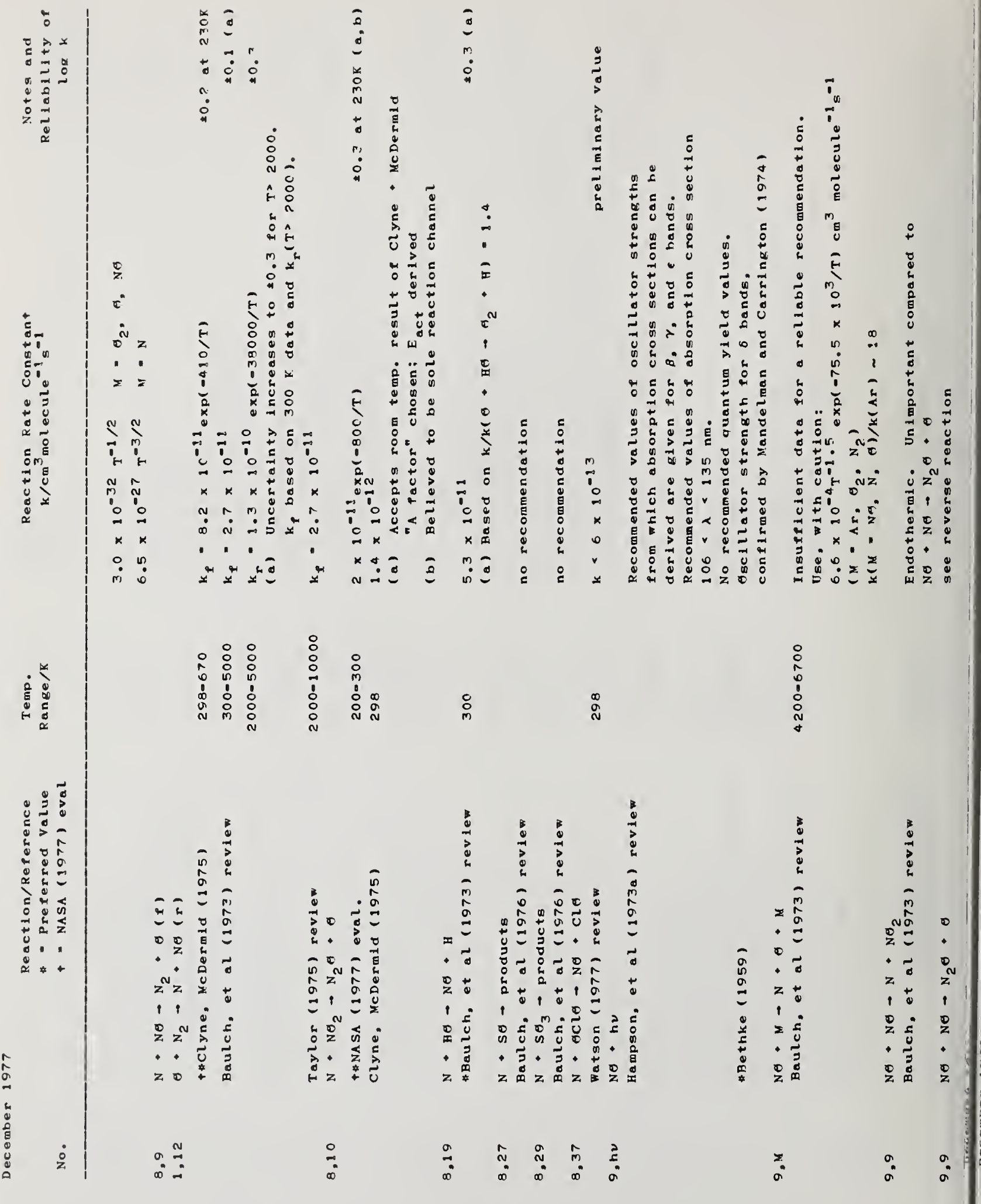




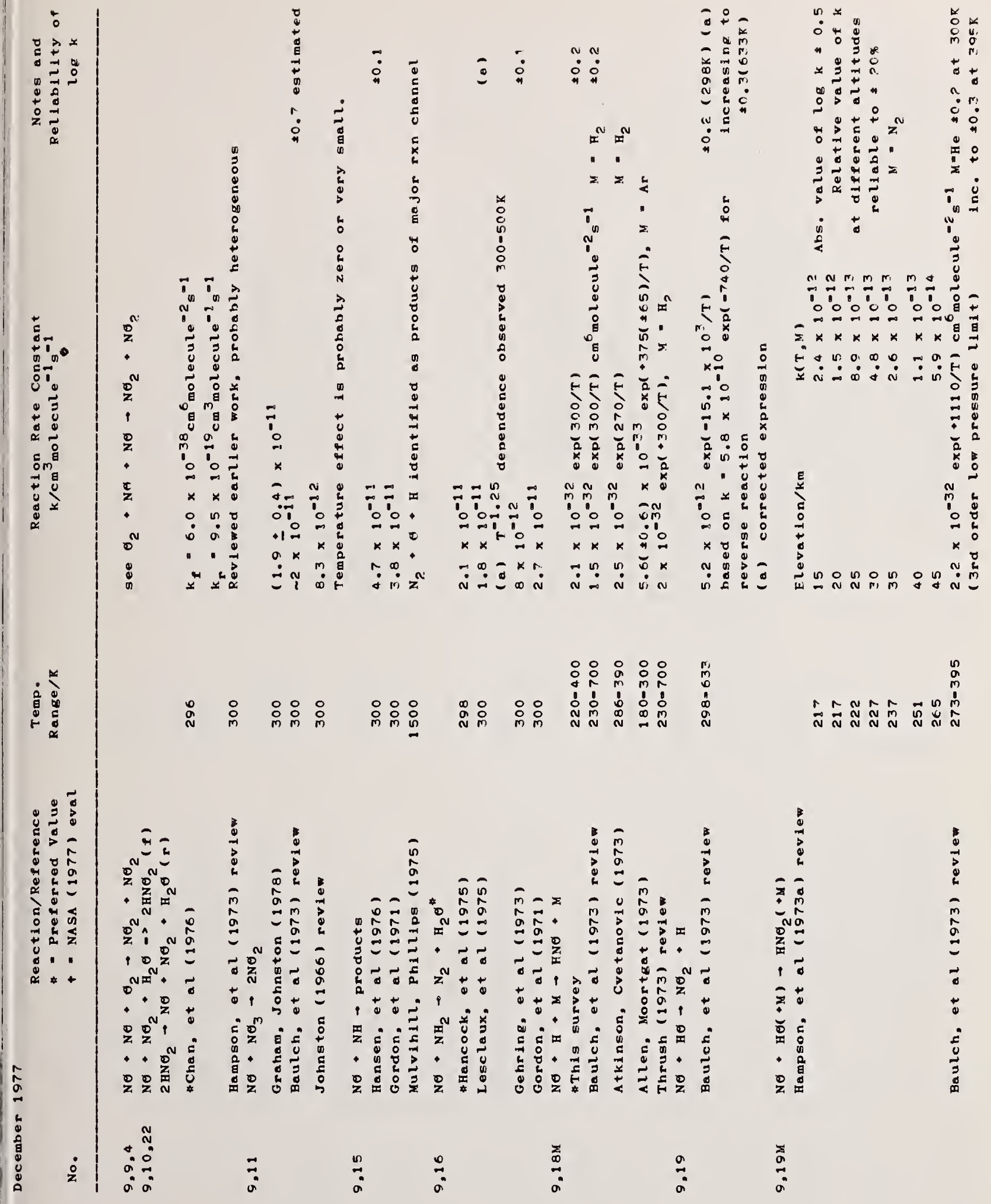




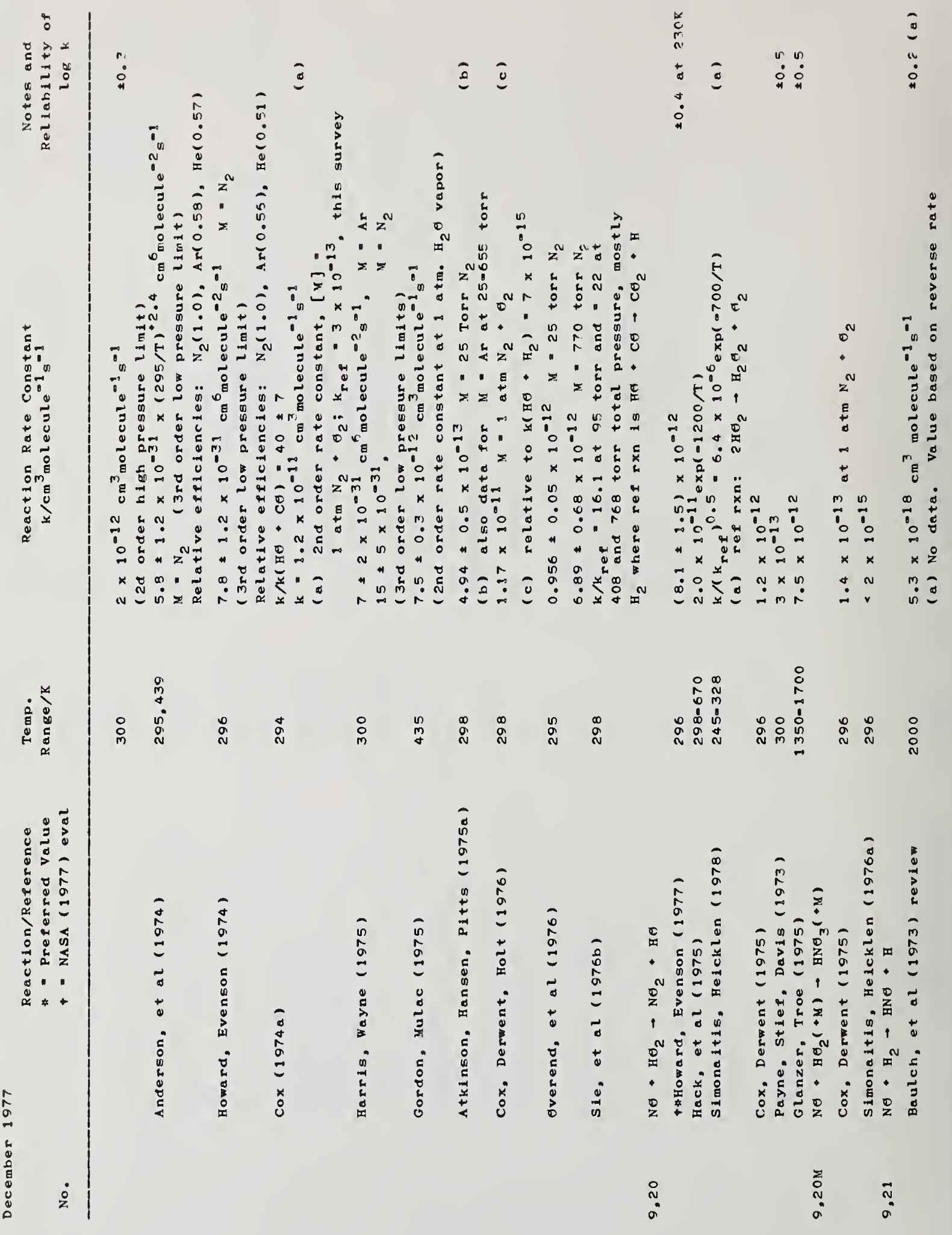




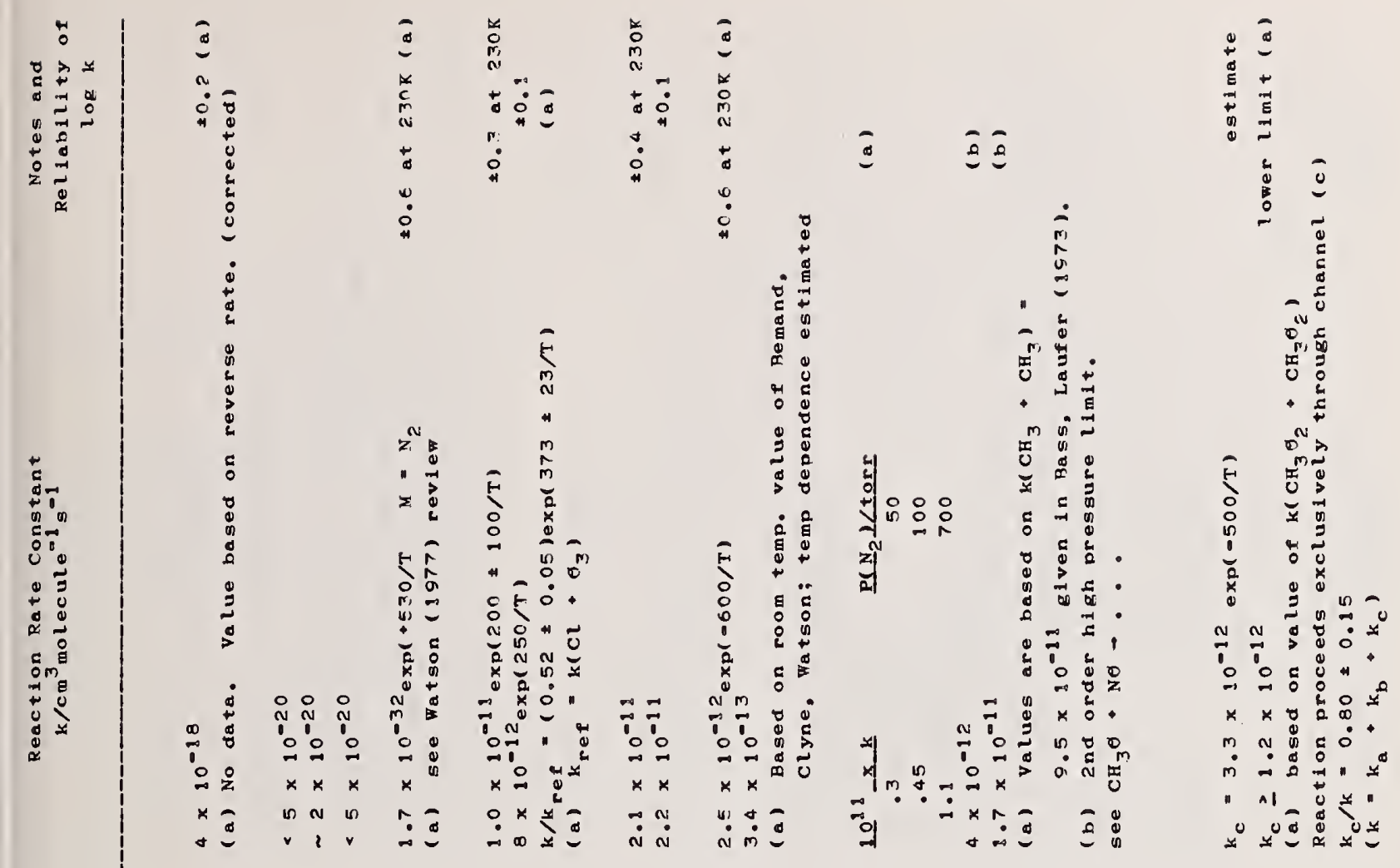

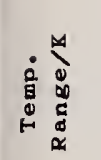

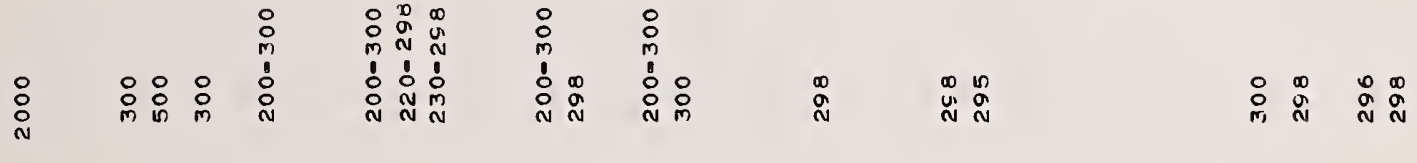

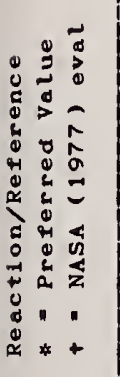

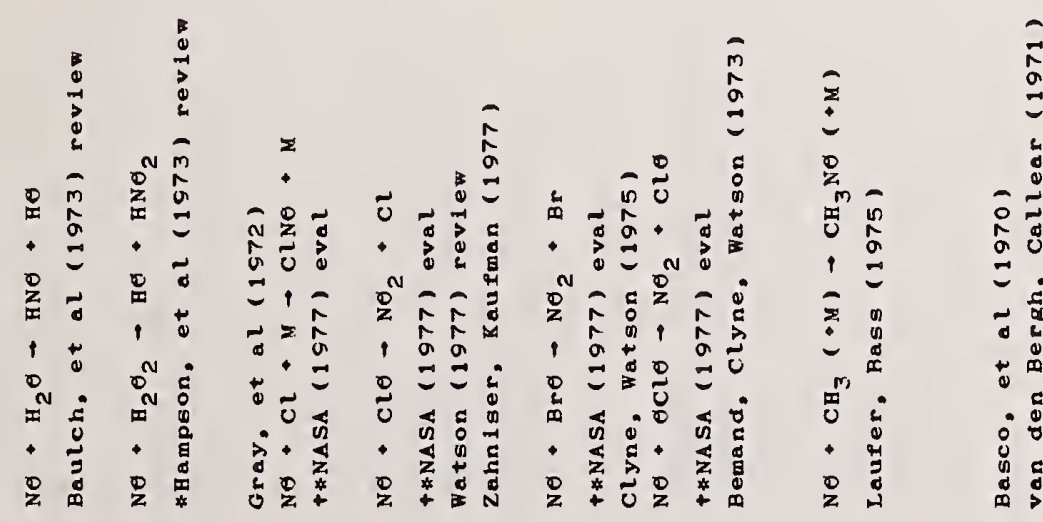

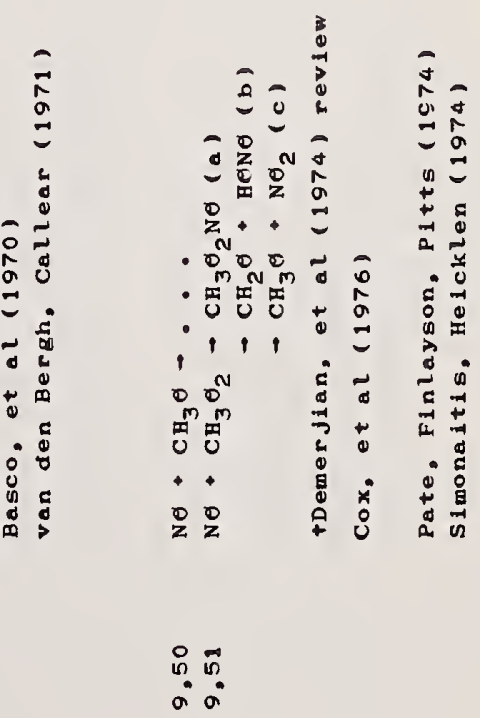




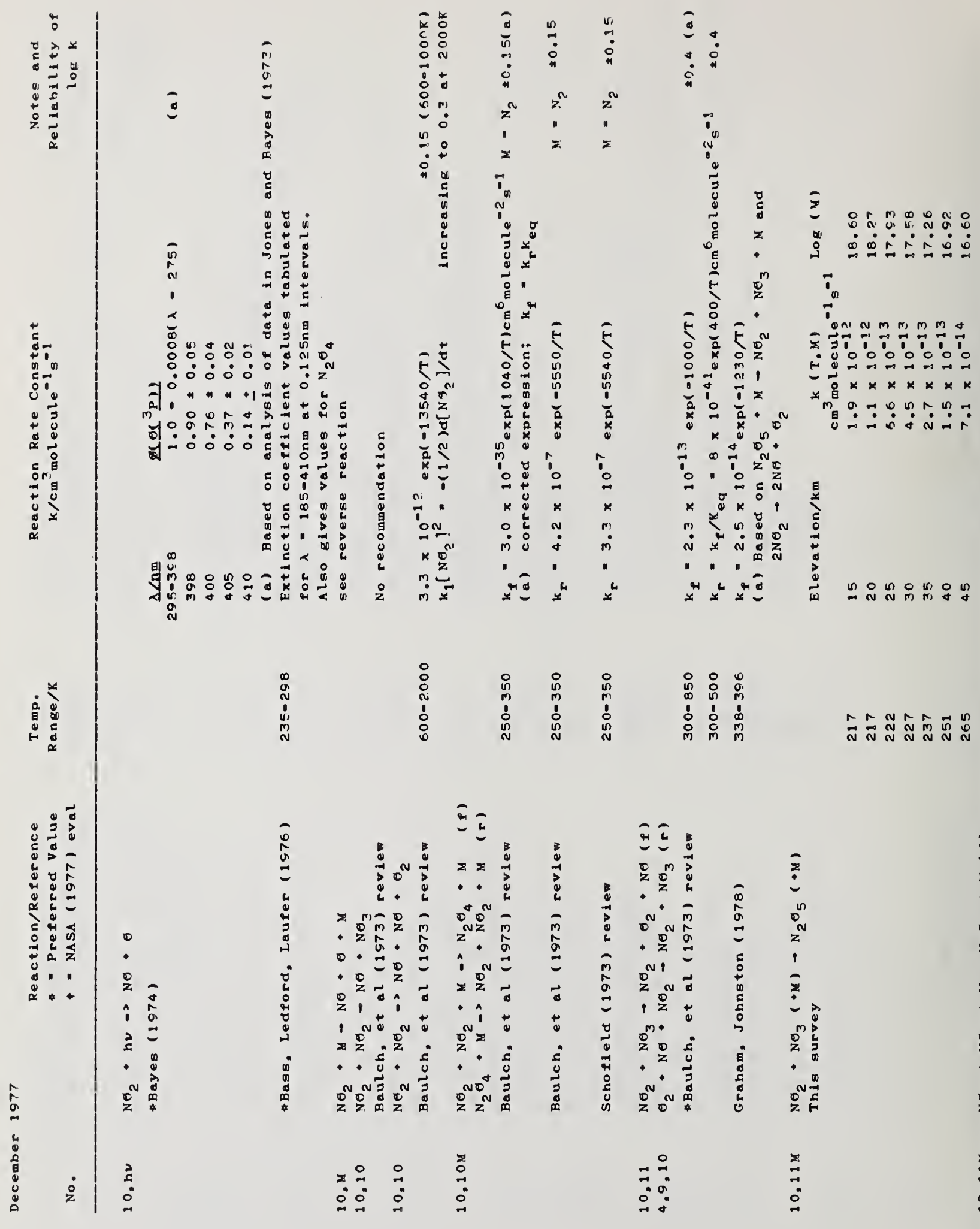

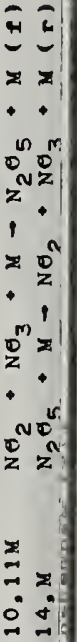




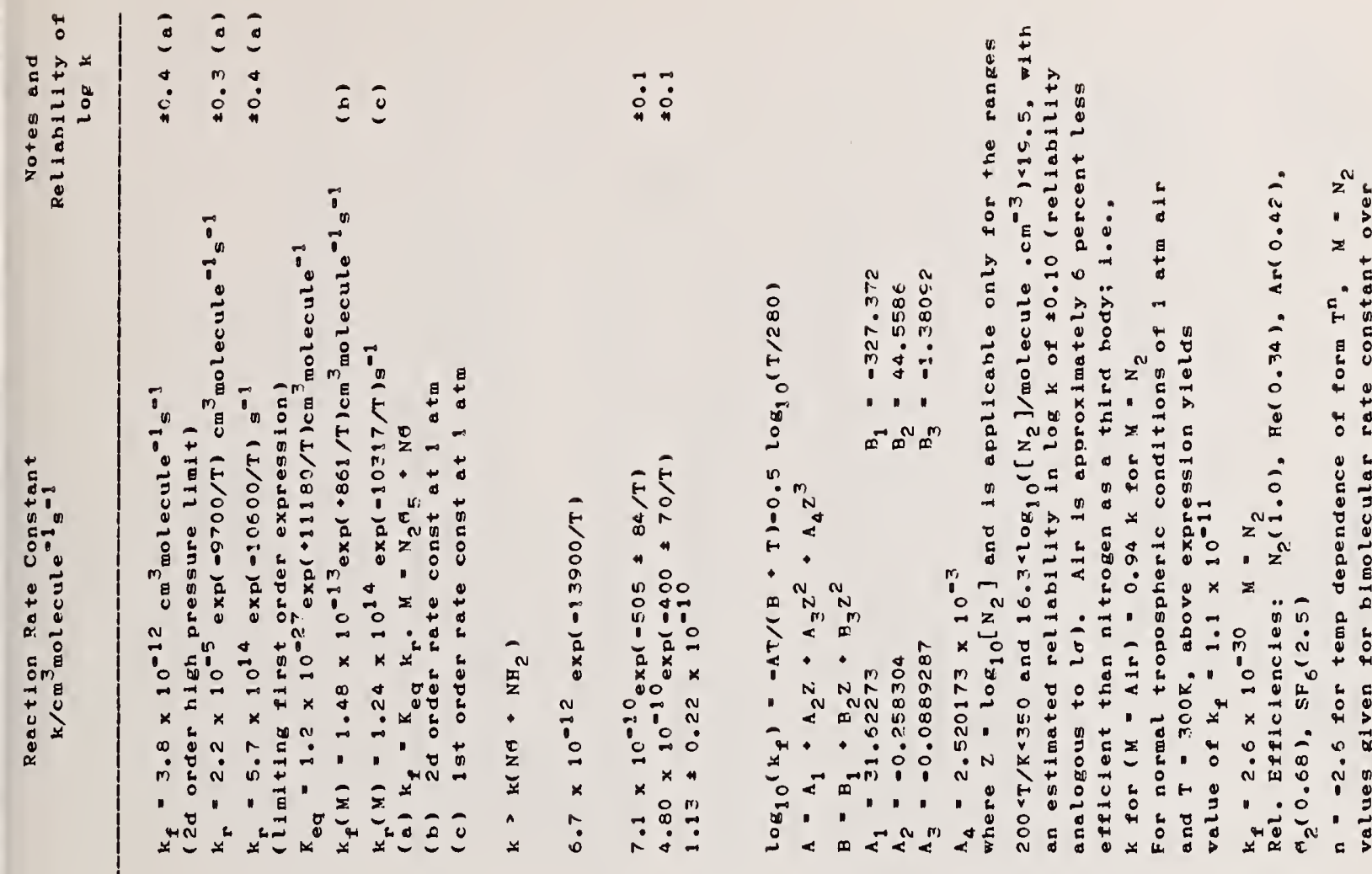

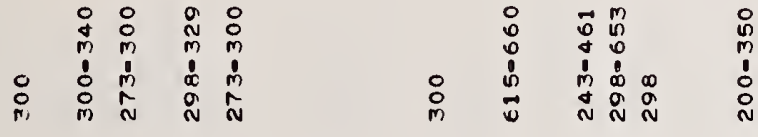

$\begin{array}{ll} & 0 \\ n & 0 \\ 0 & \vdots \\ 0 & N \\ N & N\end{array}$

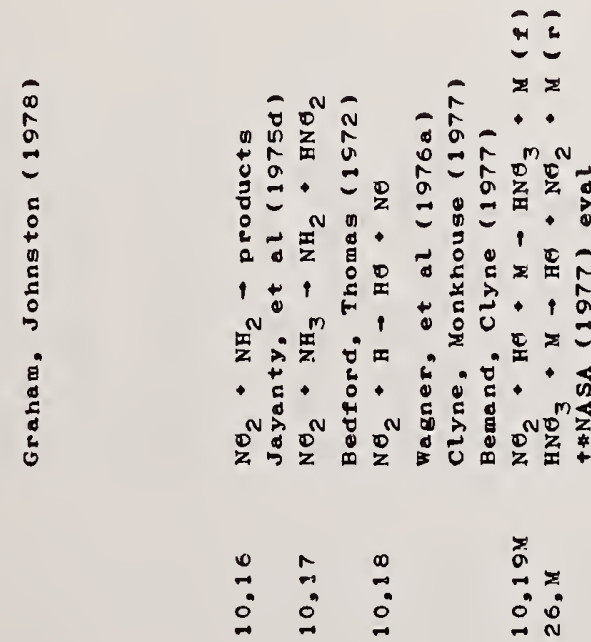




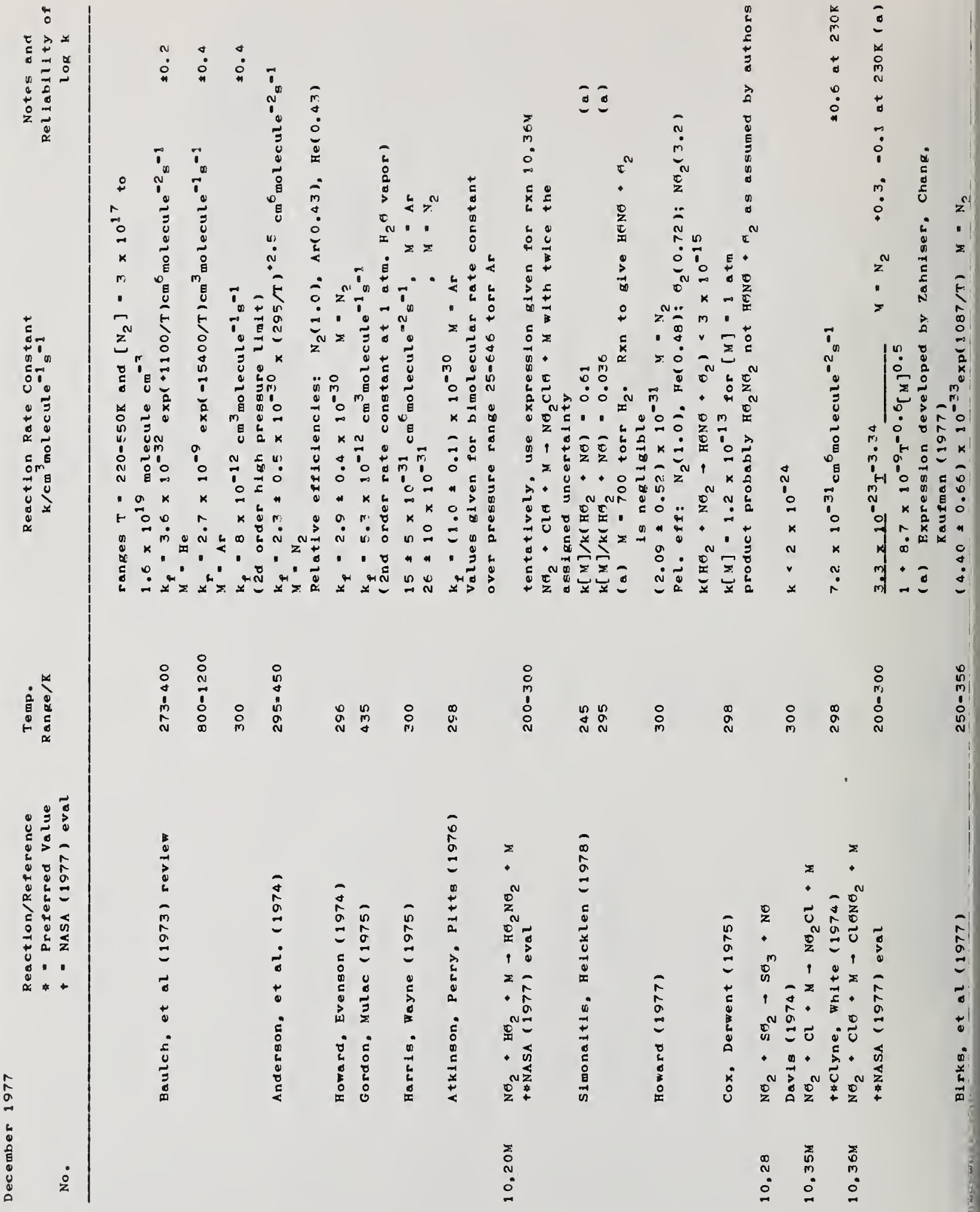



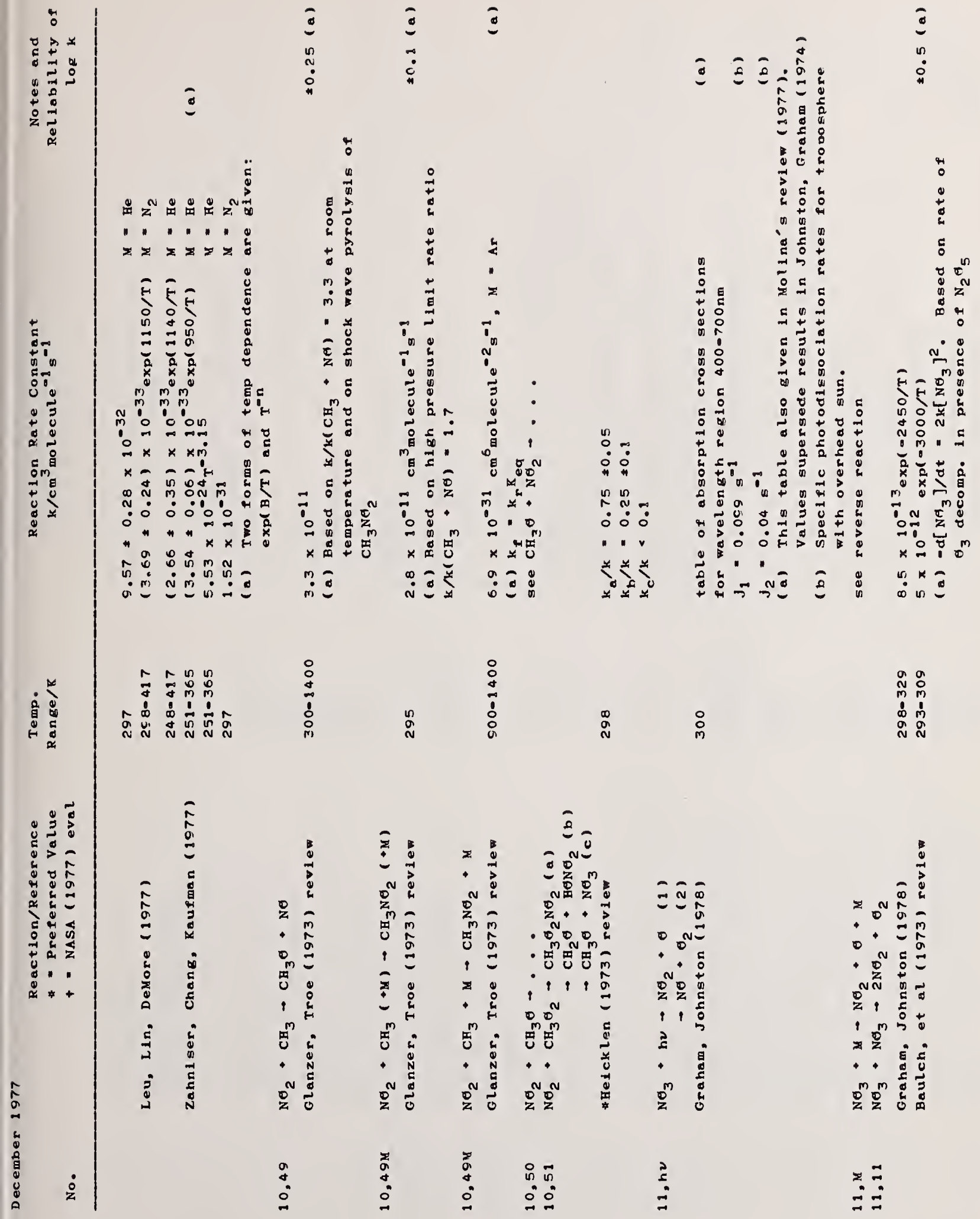


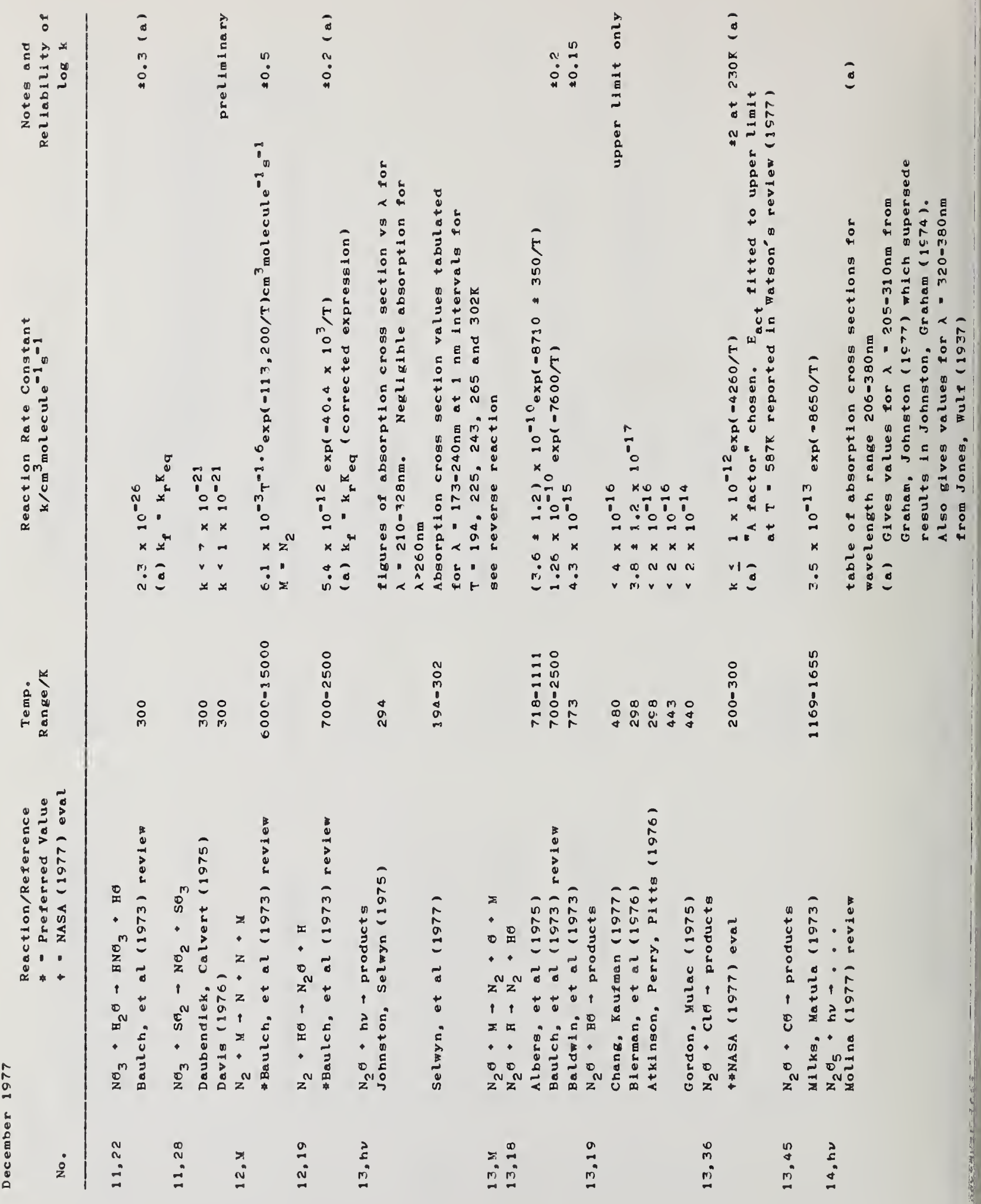




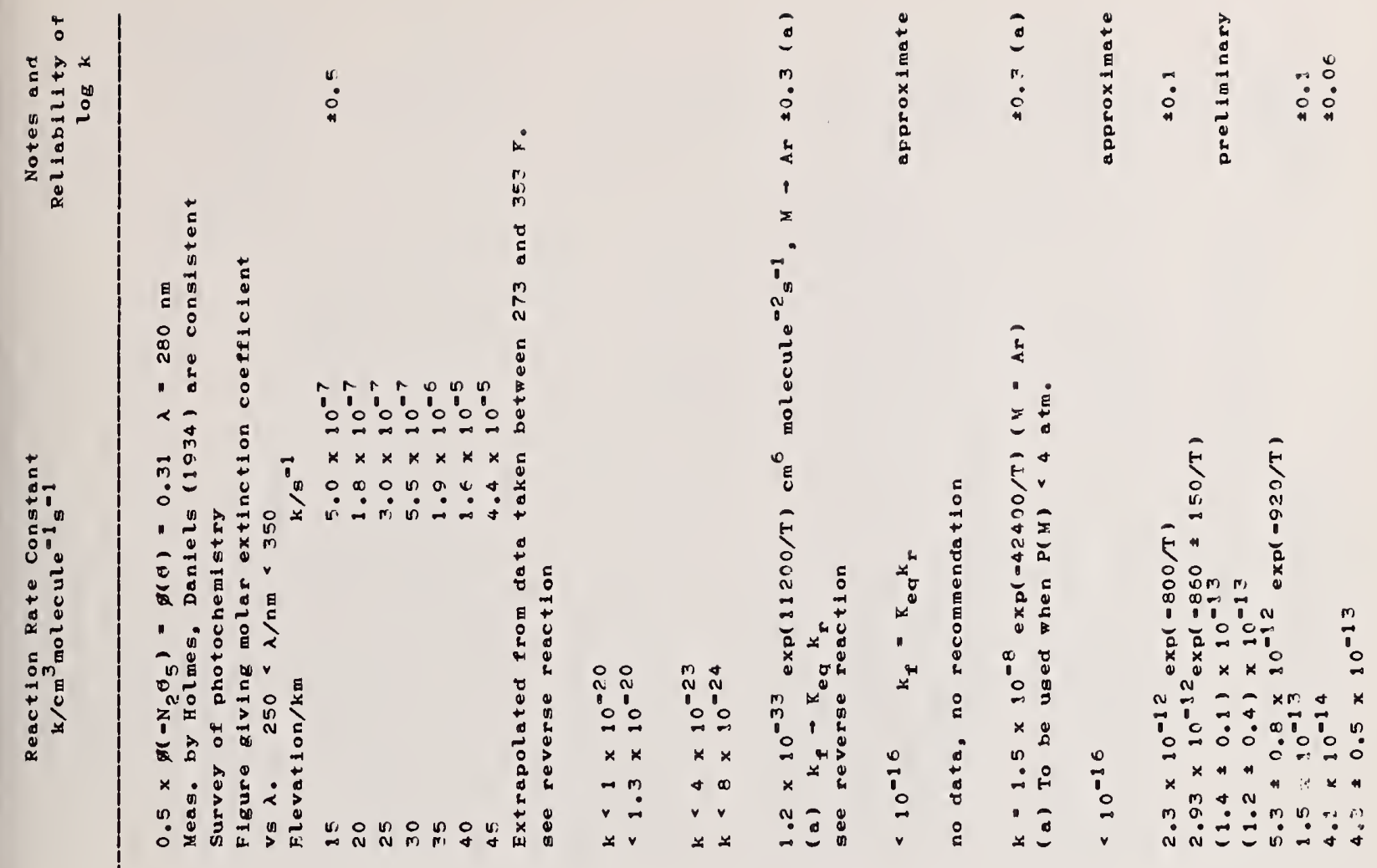

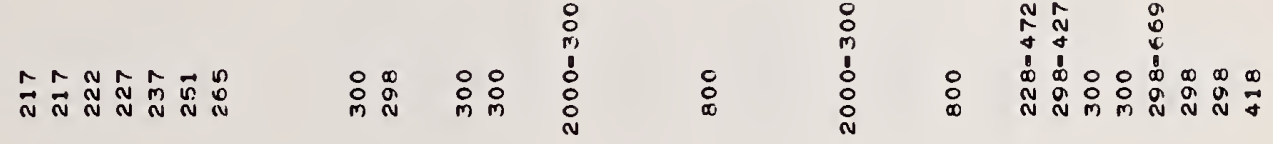

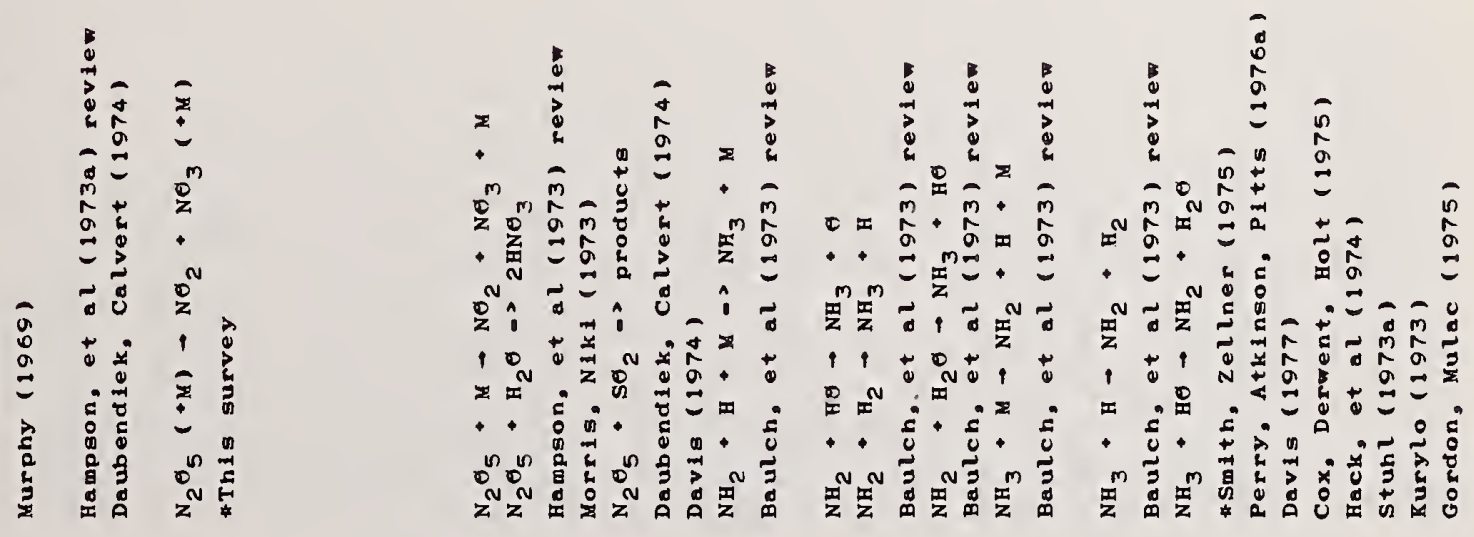

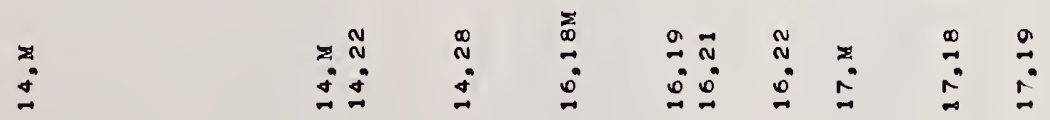




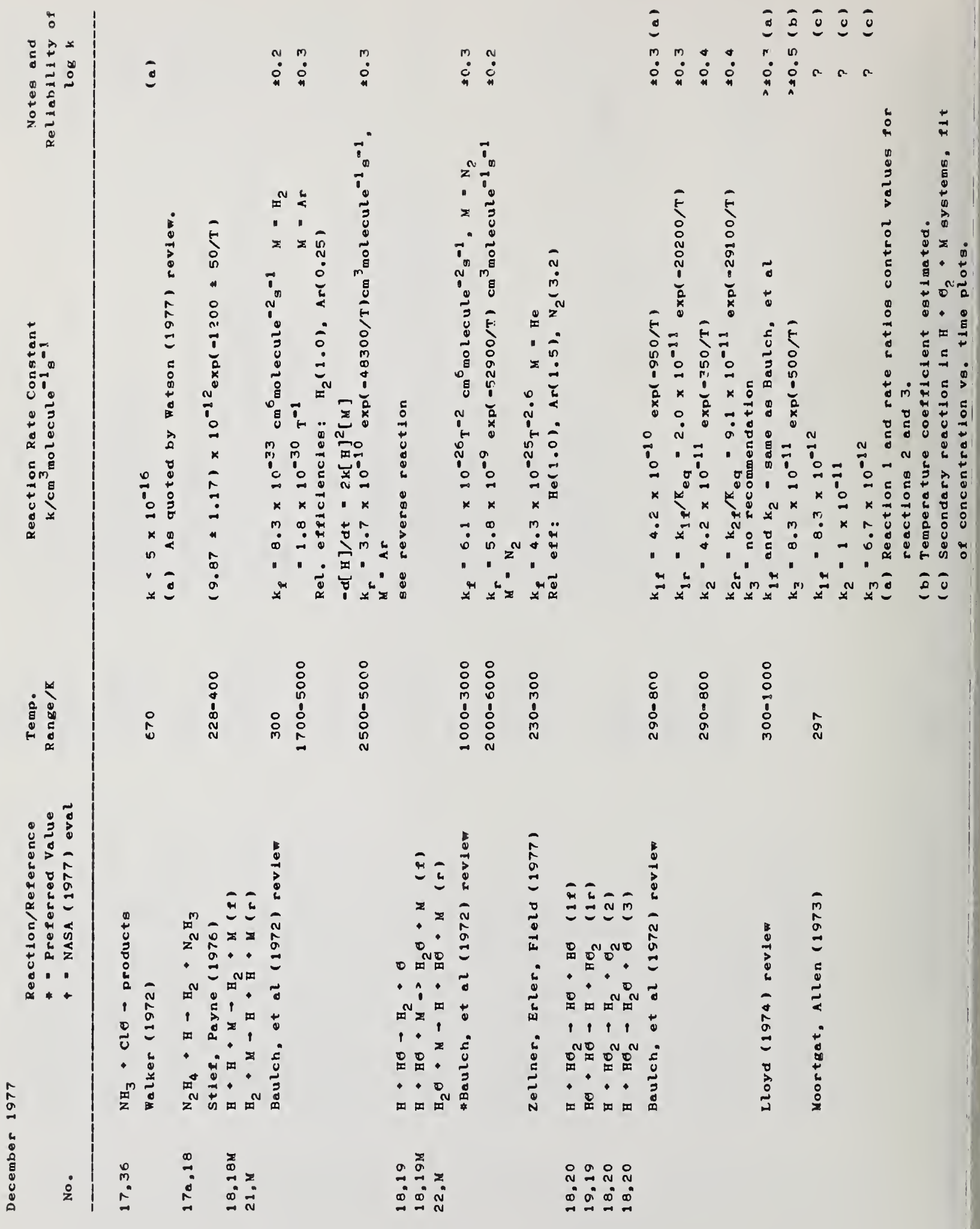




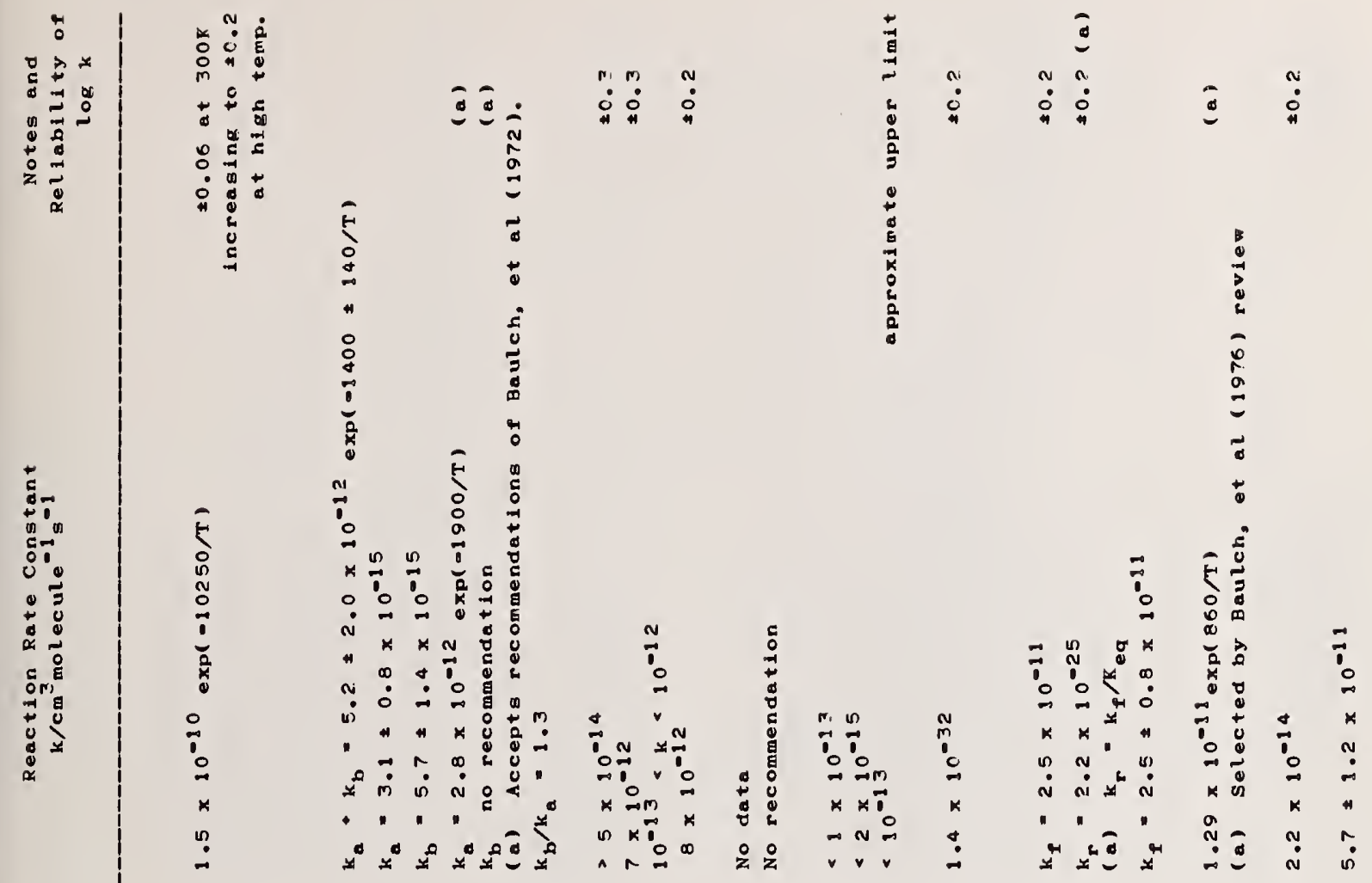

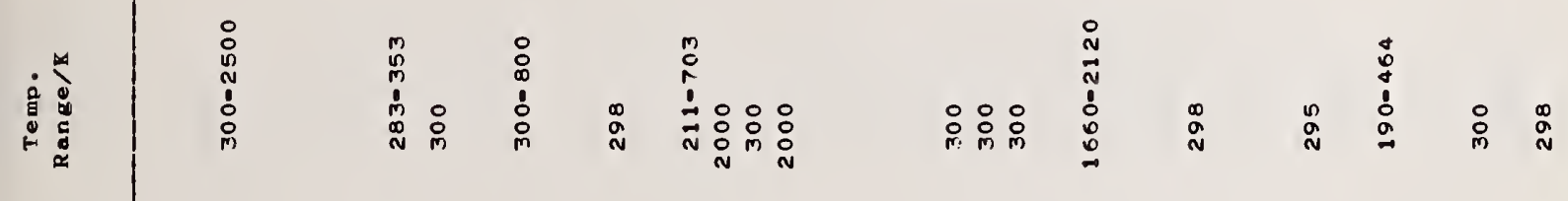

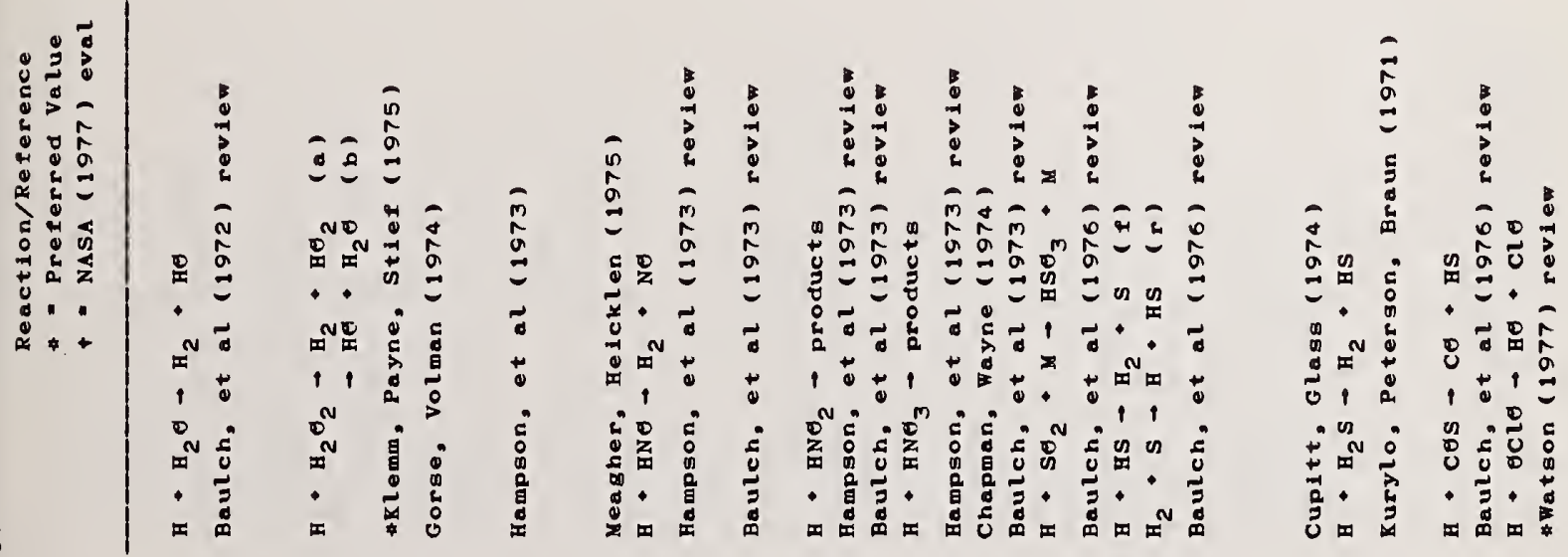

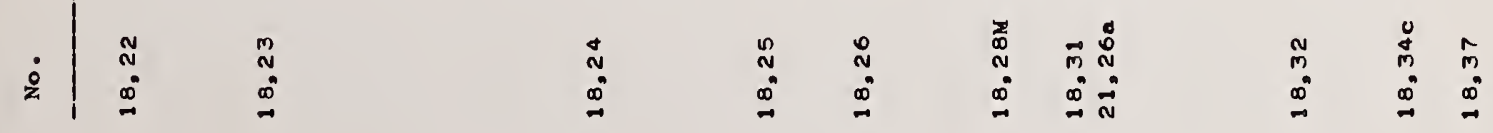




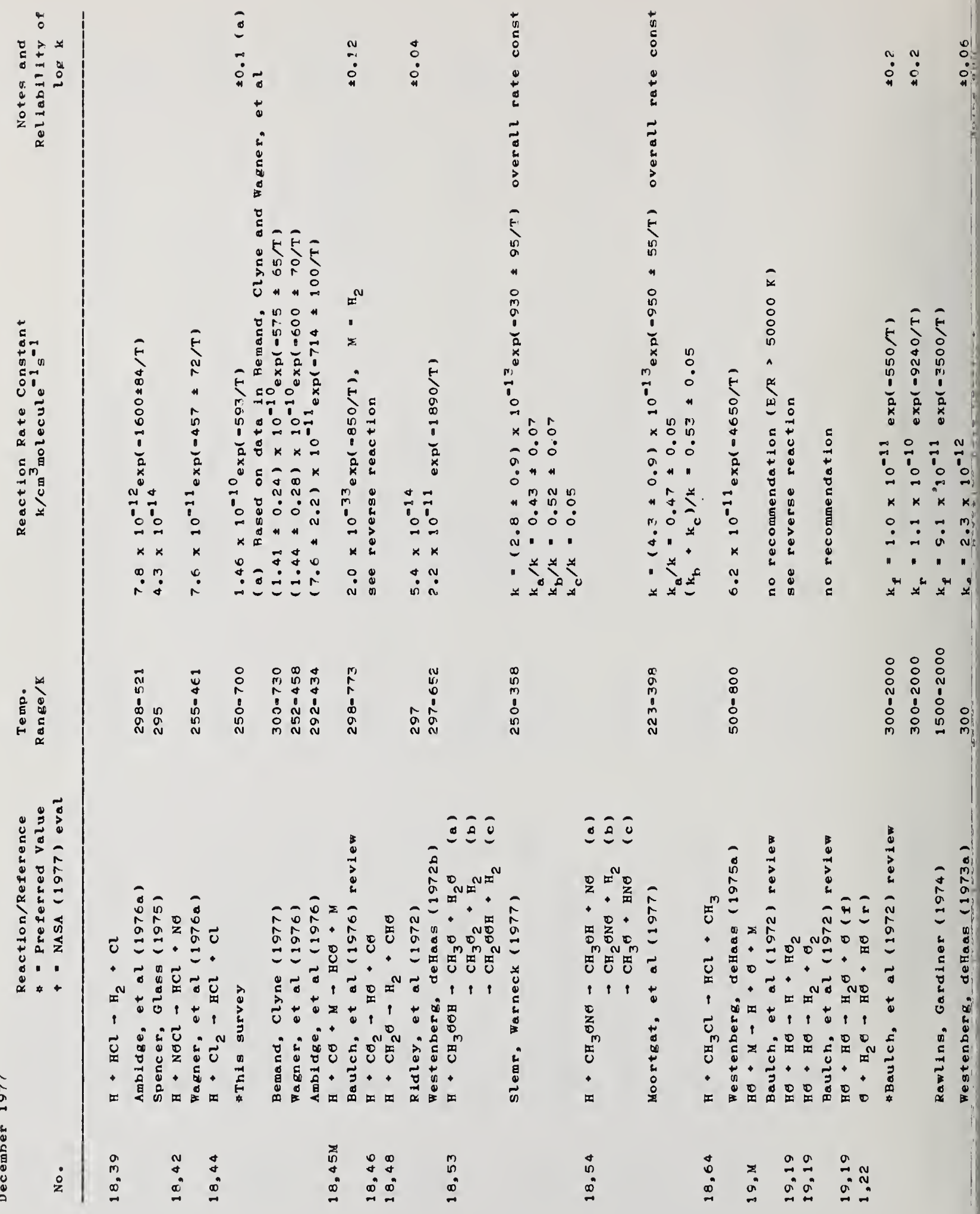



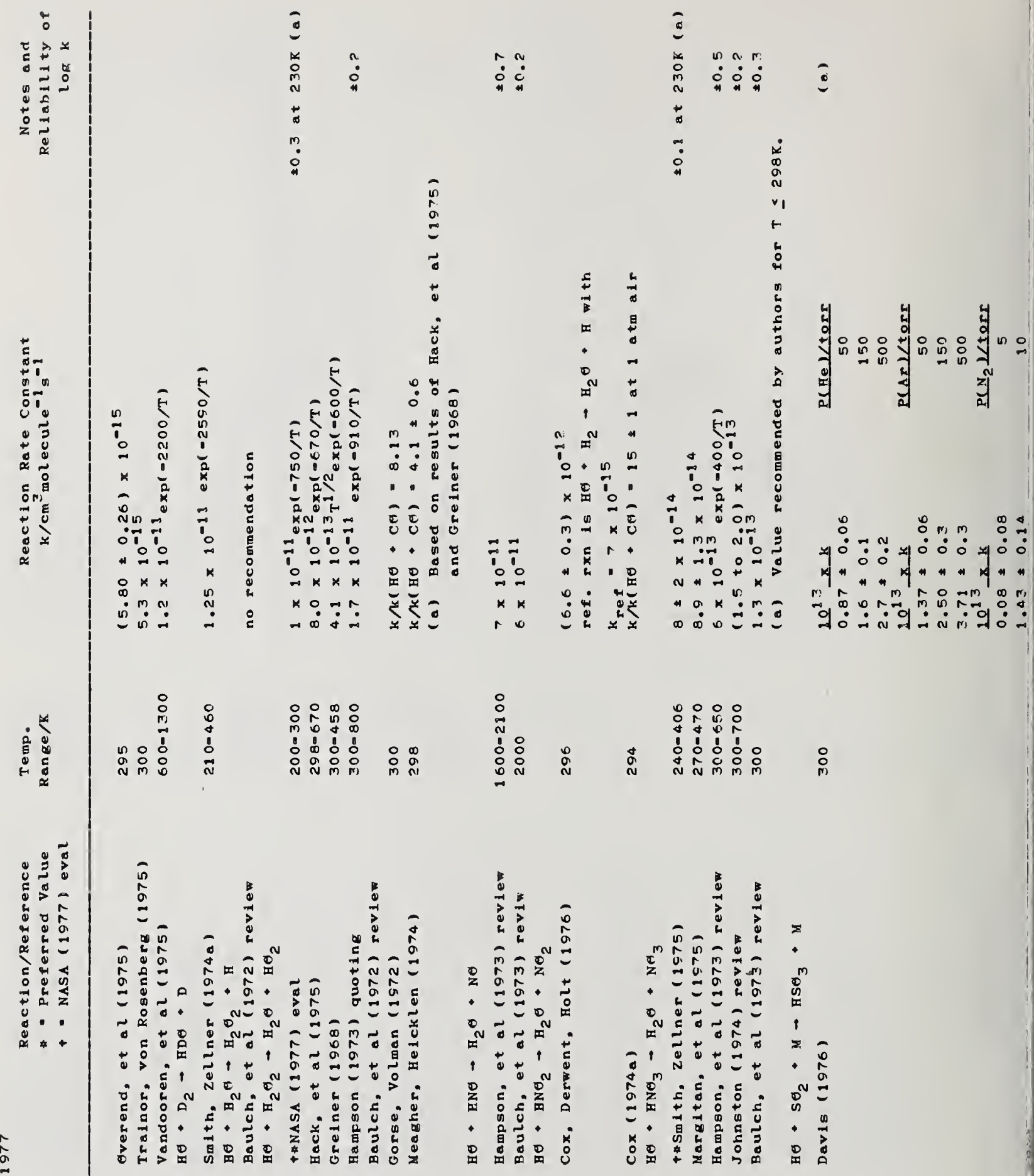

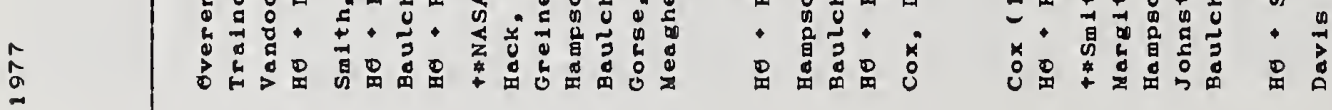




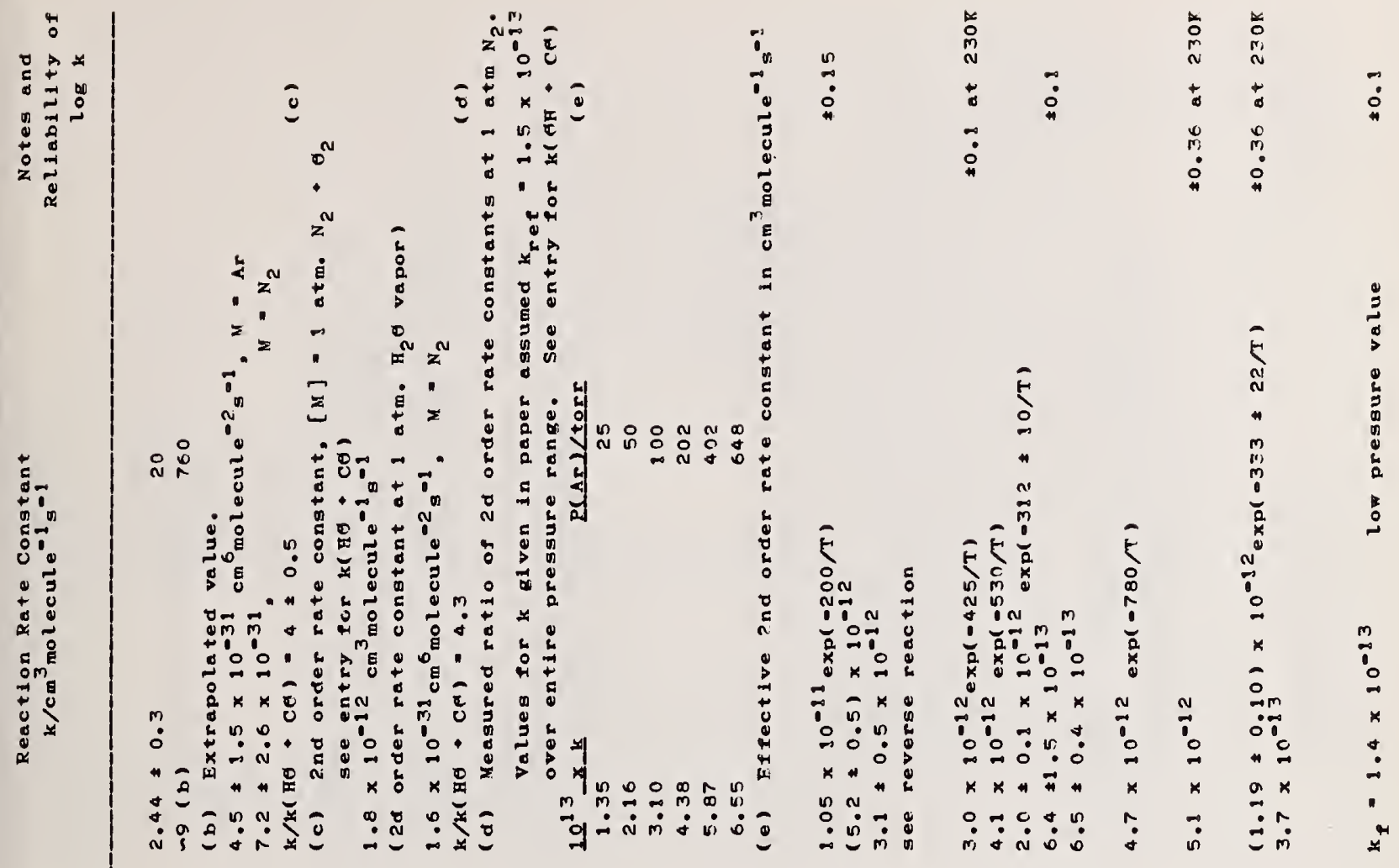

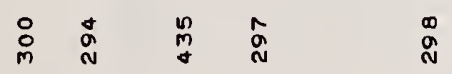

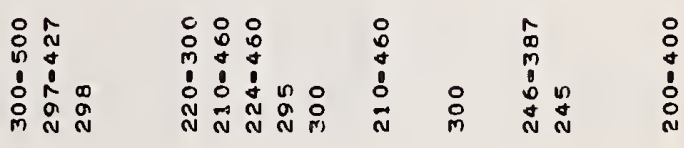

$\stackrel{\circ}{\circ}$

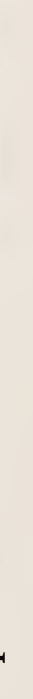




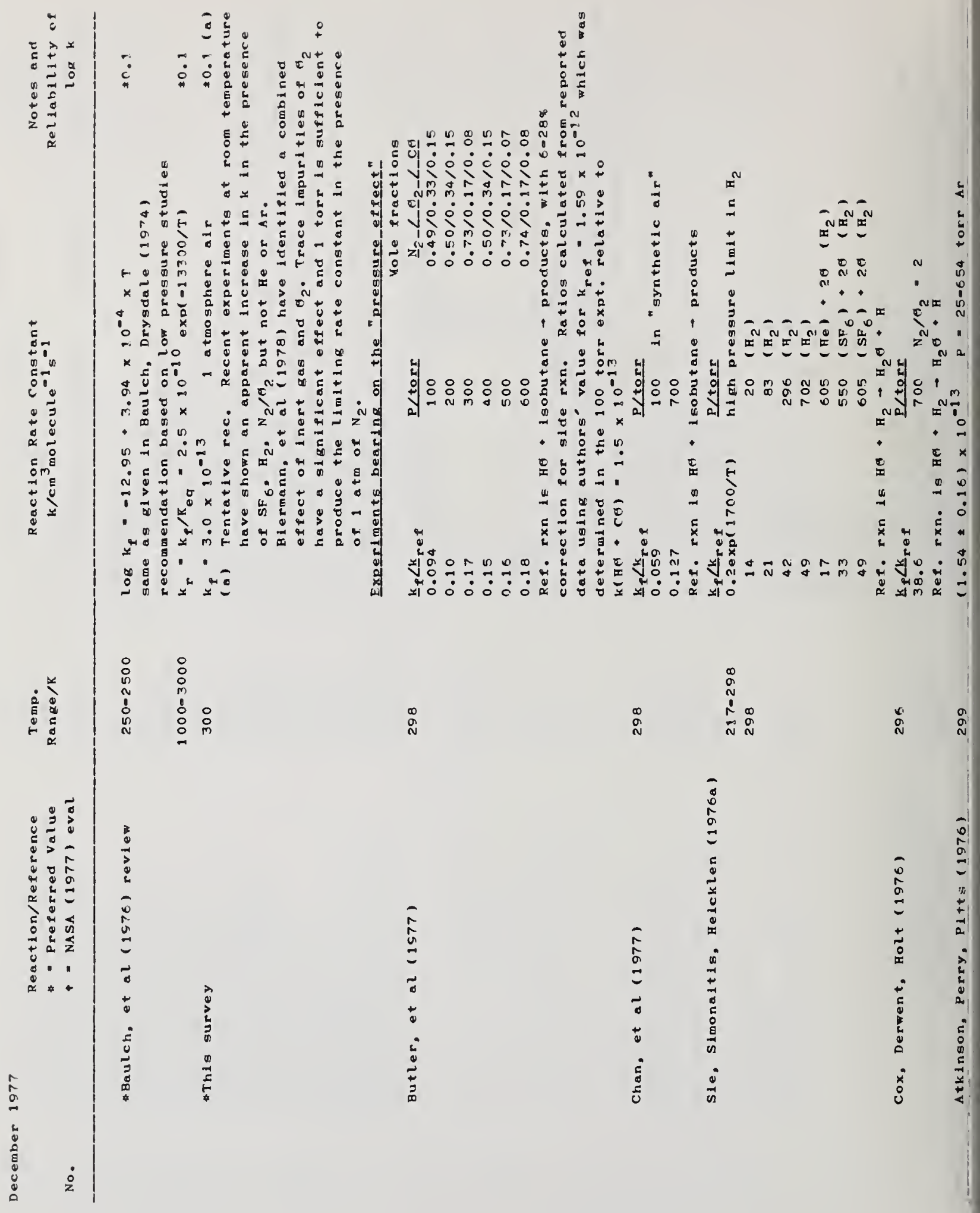




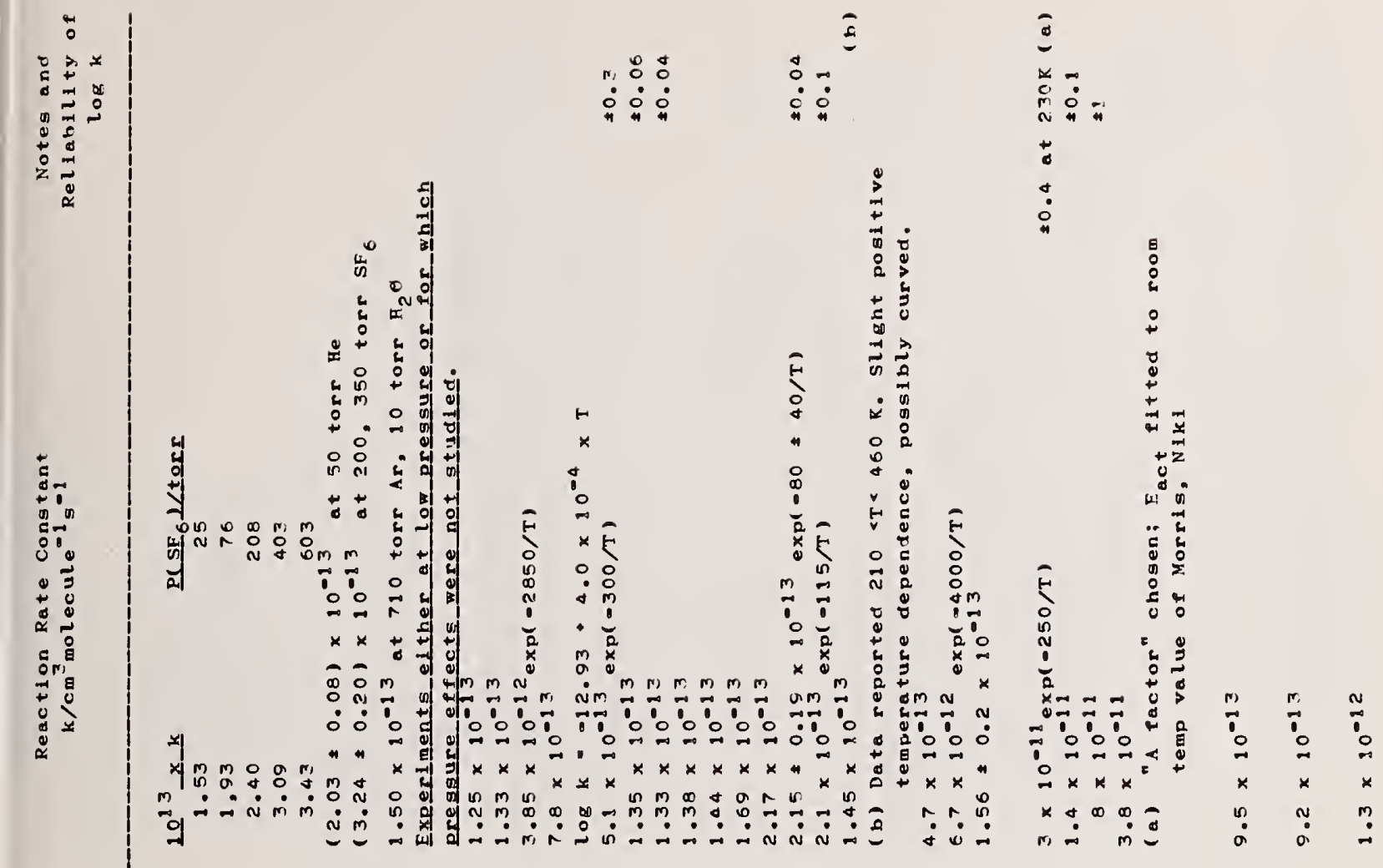

$\stackrel{0}{\circ}$

$\therefore: ㅇ ㅛ ㅇ ㅛ$

a man

m

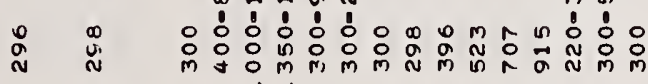

$\stackrel{a}{a}$
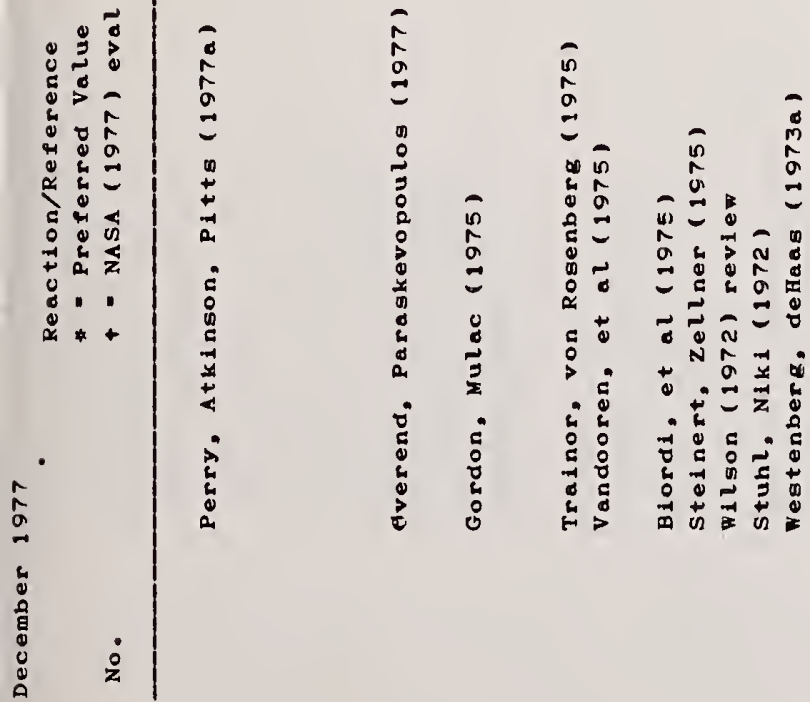

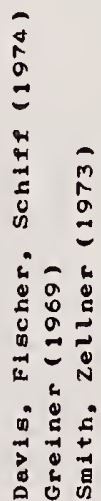

0
$:$

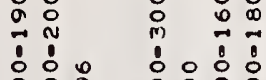

$\stackrel{\infty}{\circ}$

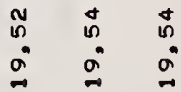




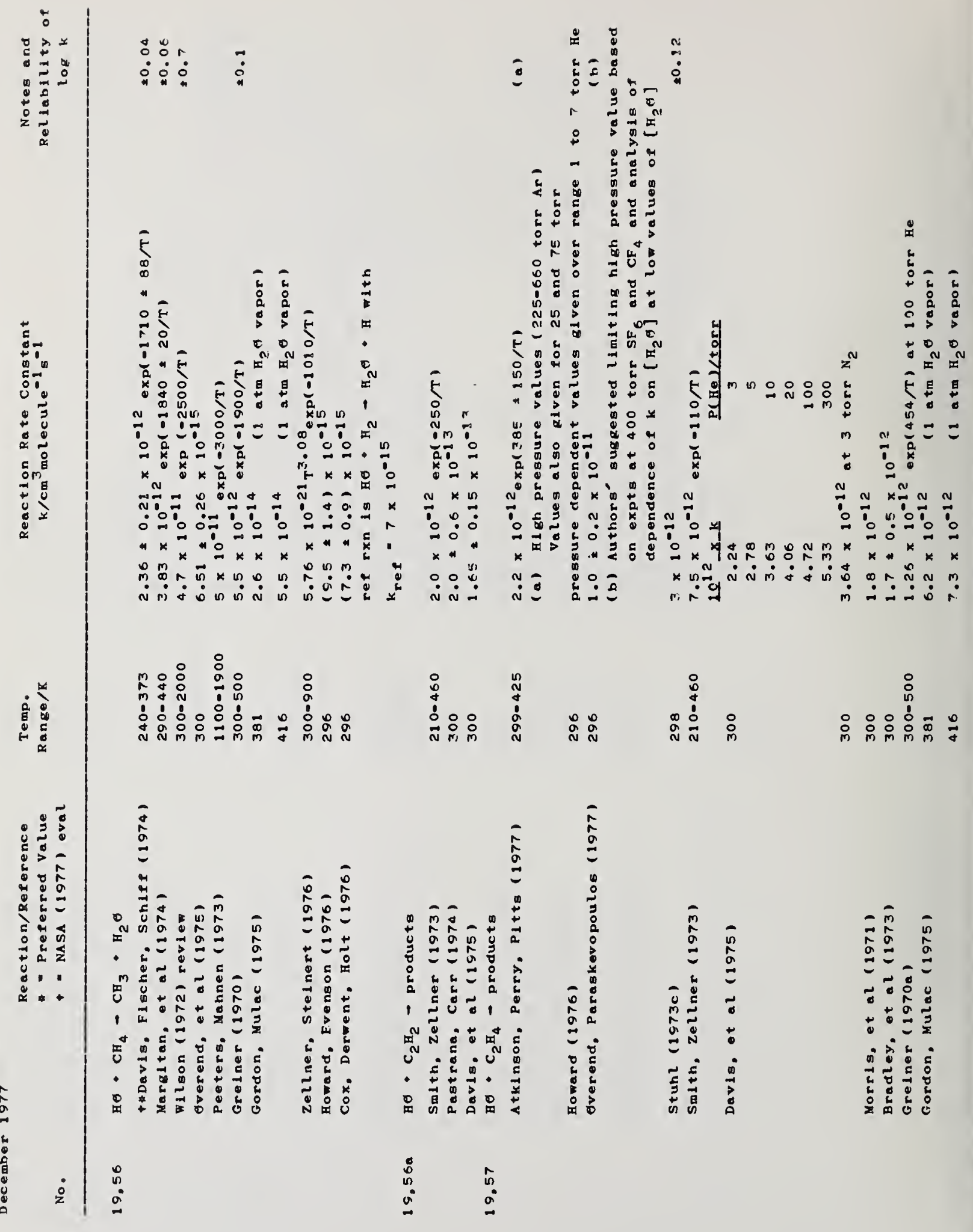




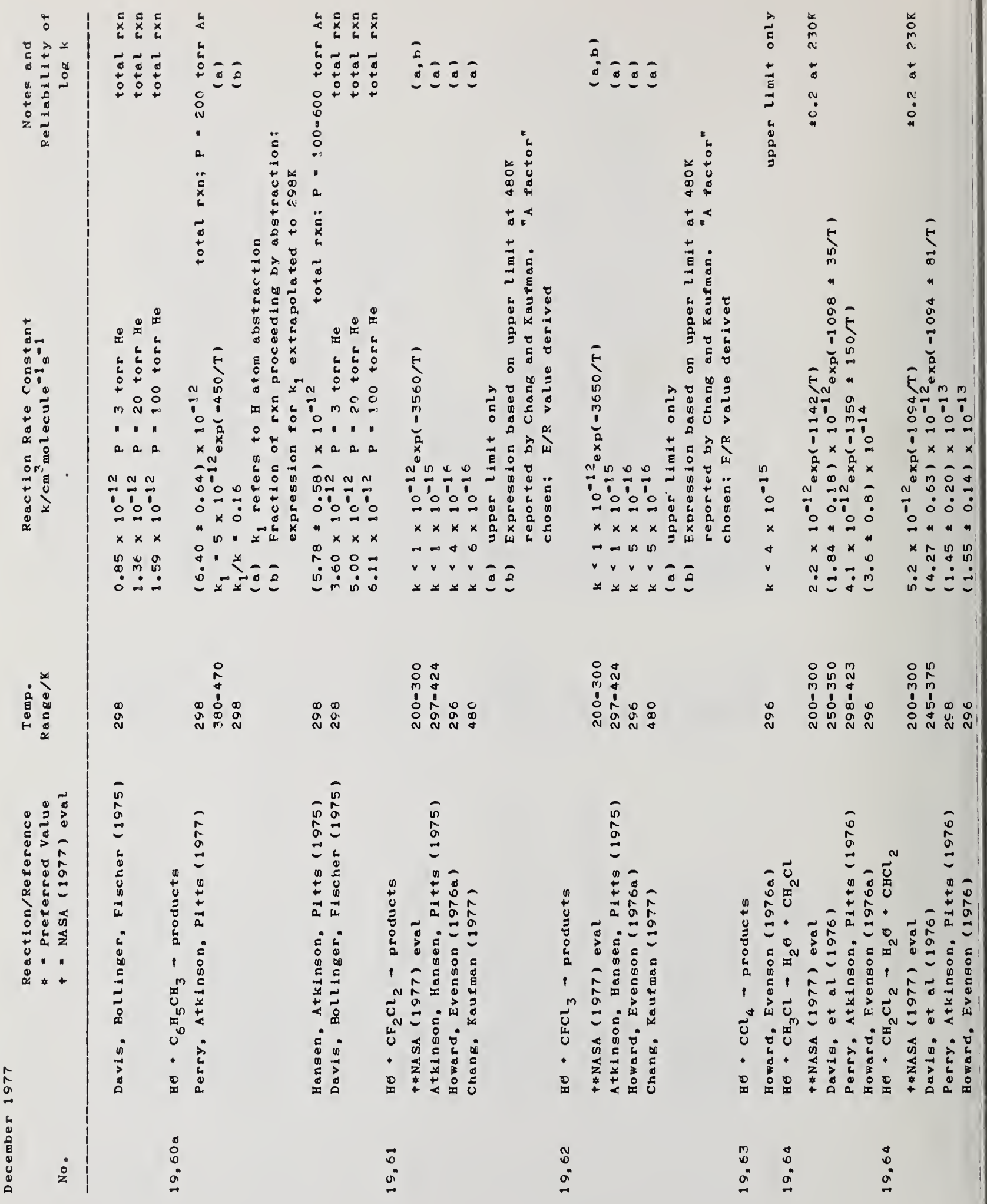




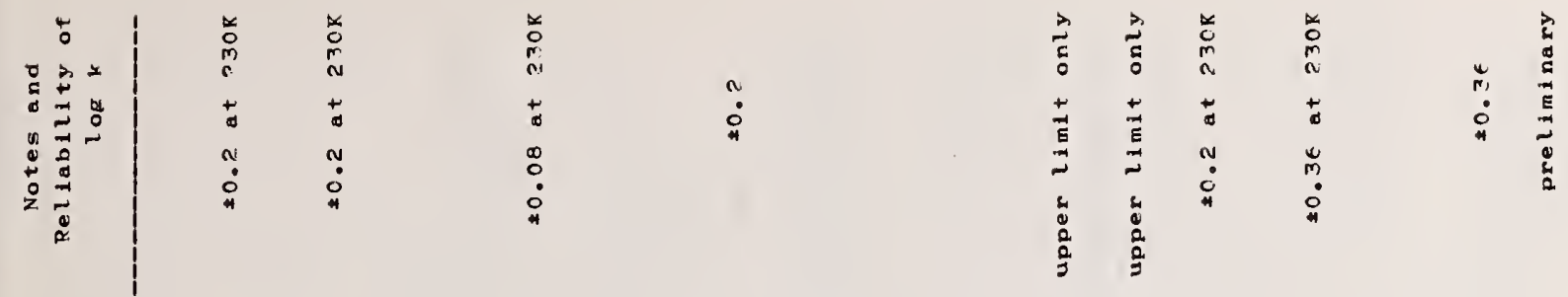

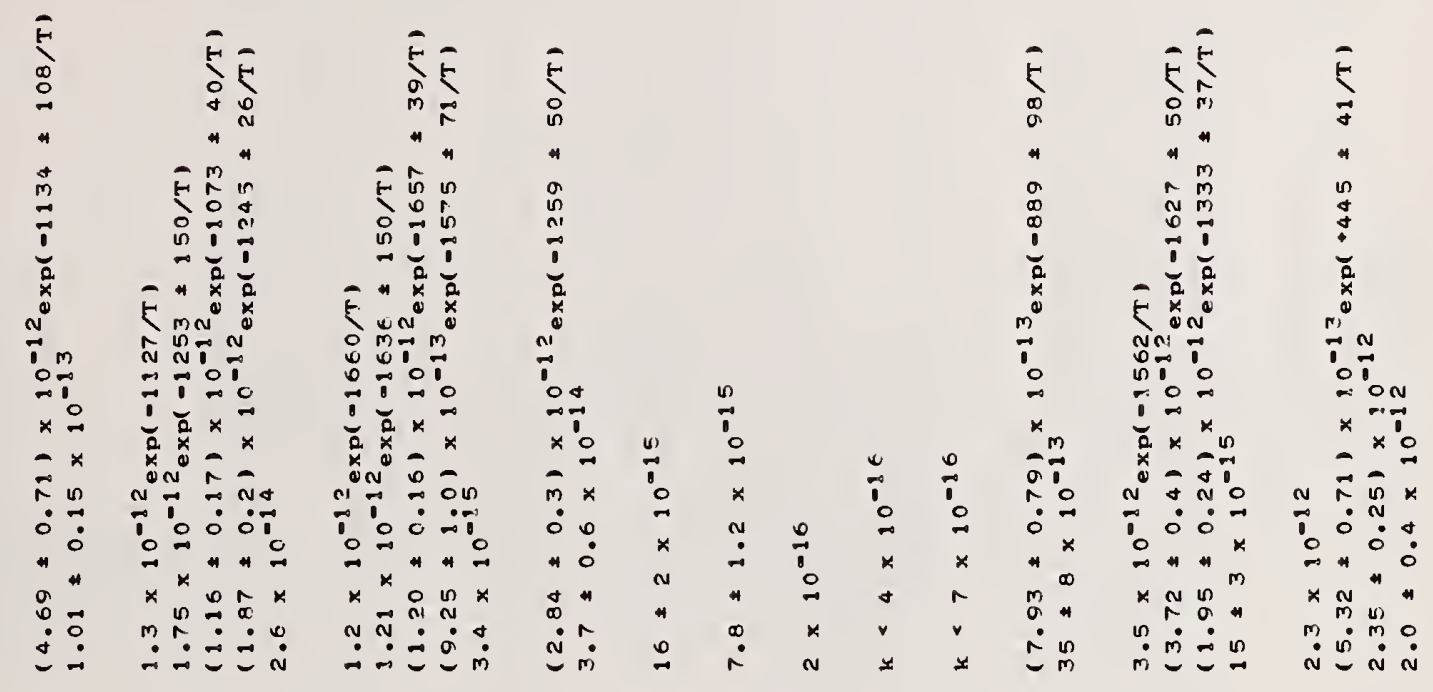

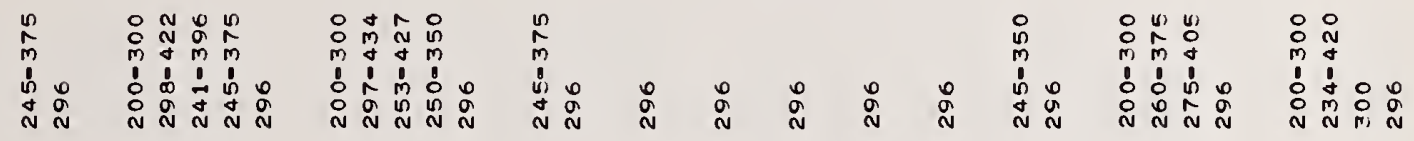

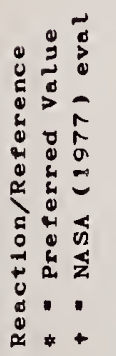

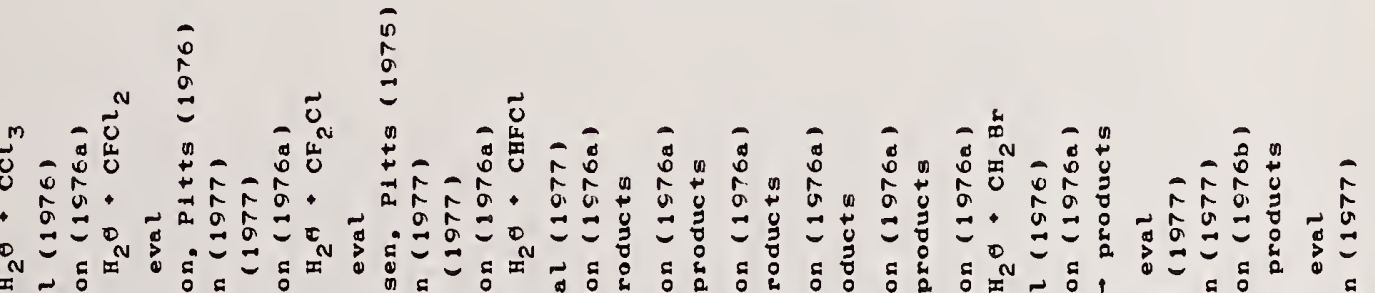

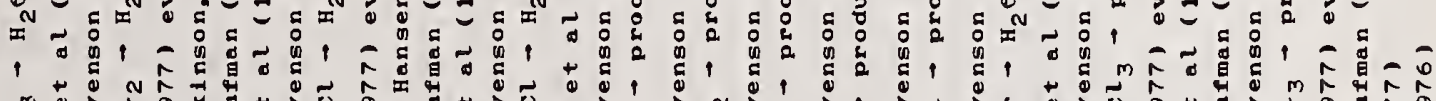
m.

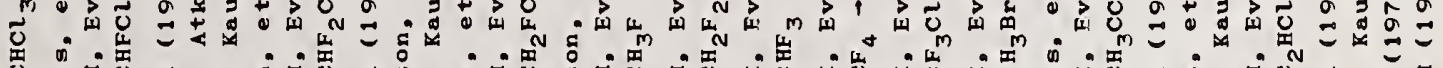
겅

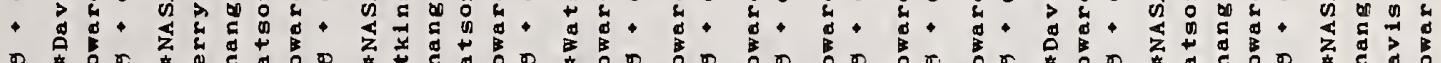

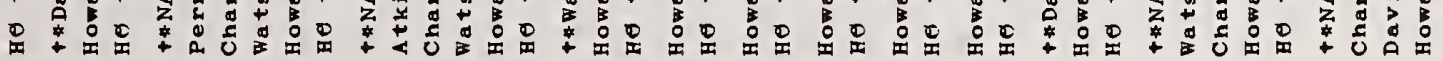

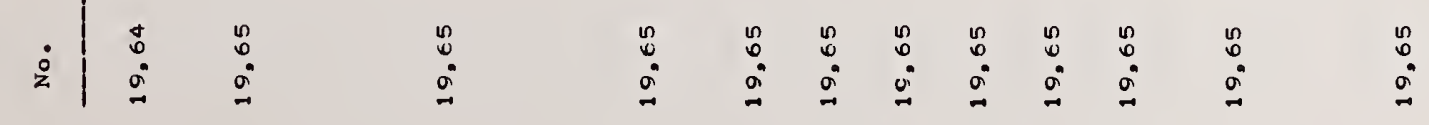



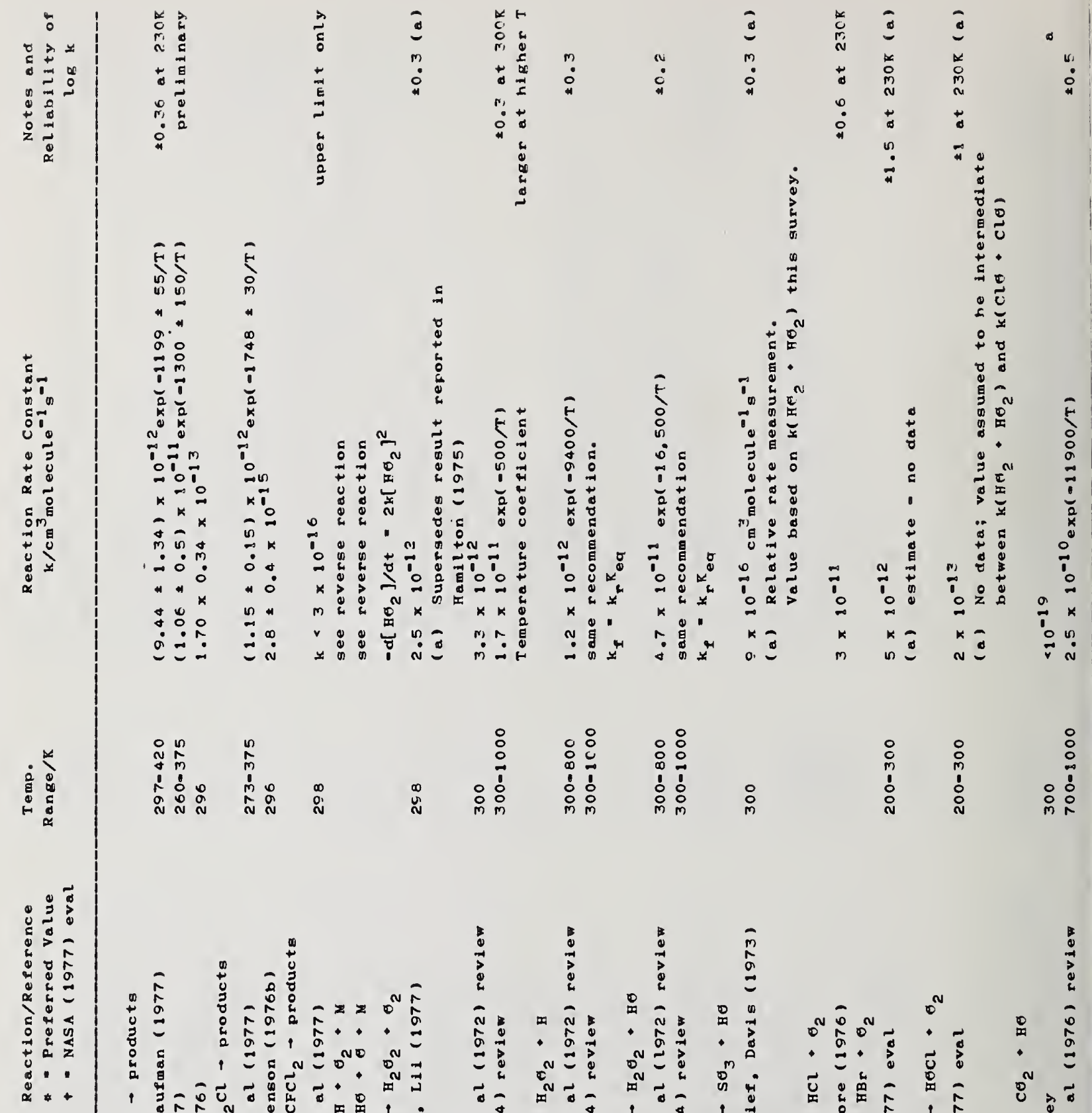

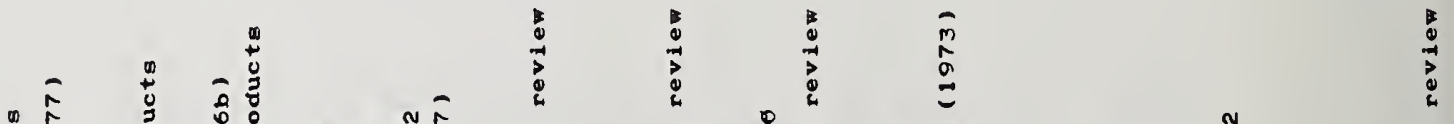

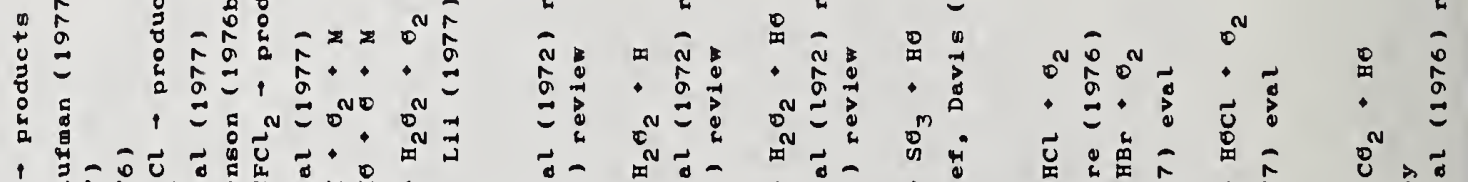
U. ऊ $0^{n}$

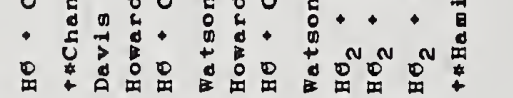

$$
5 \quad 4
$$

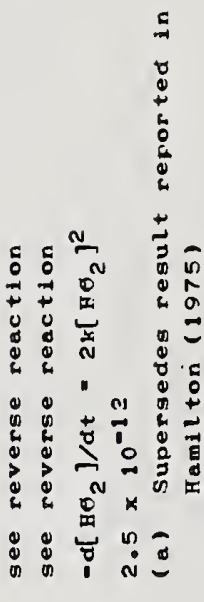

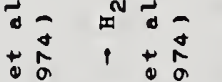

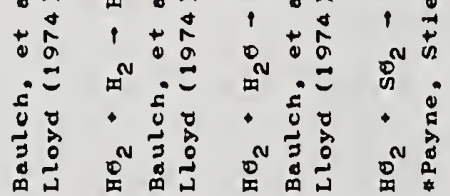

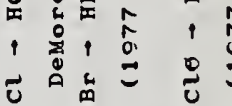

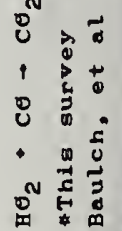

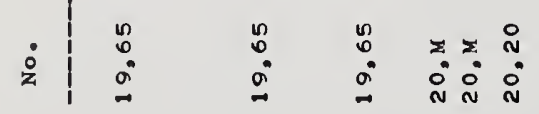

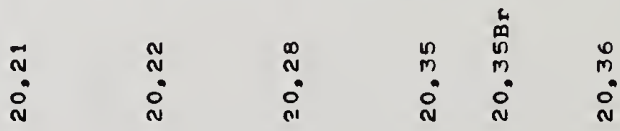
$\stackrel{n}{\dot{0}}$ 


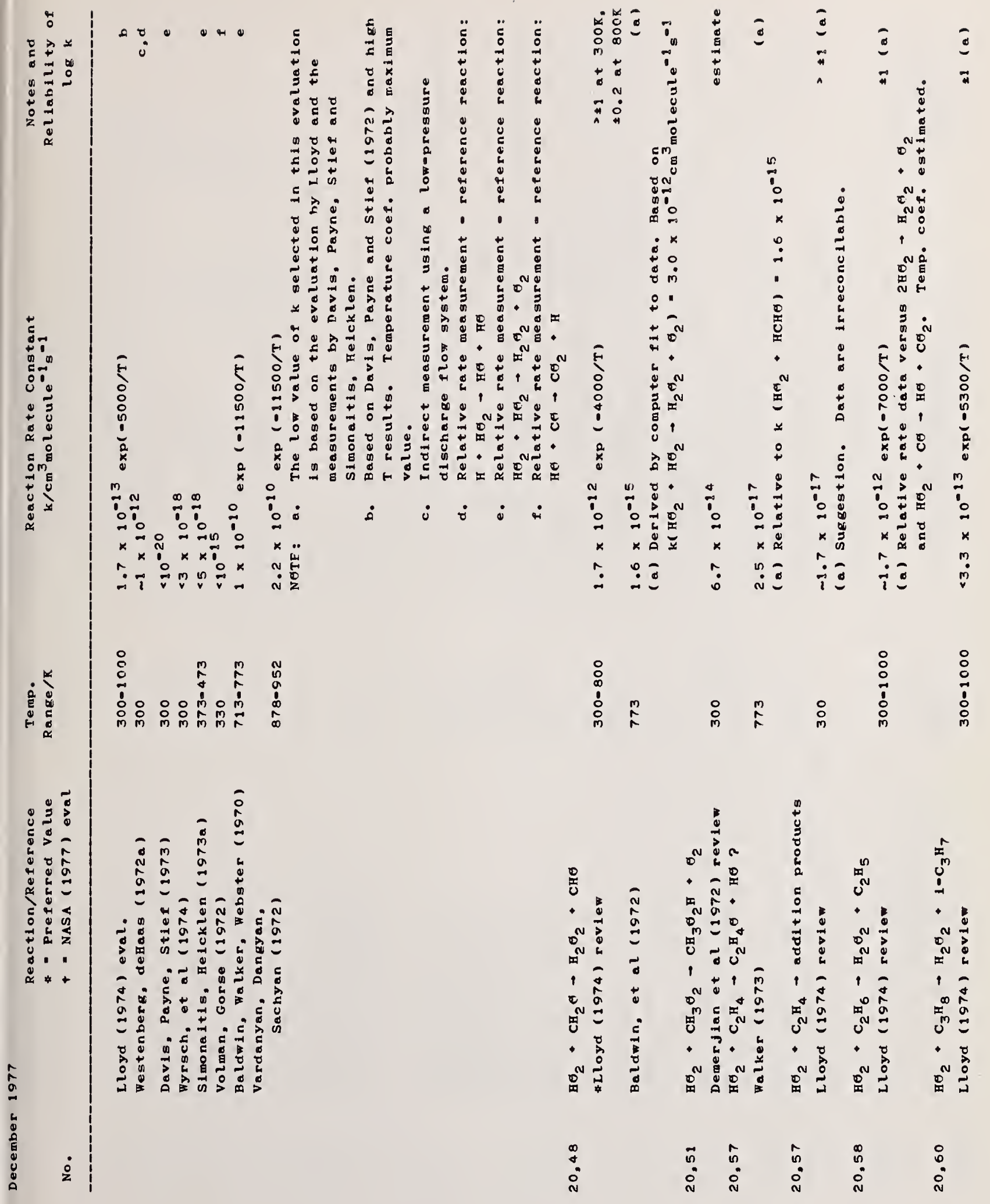




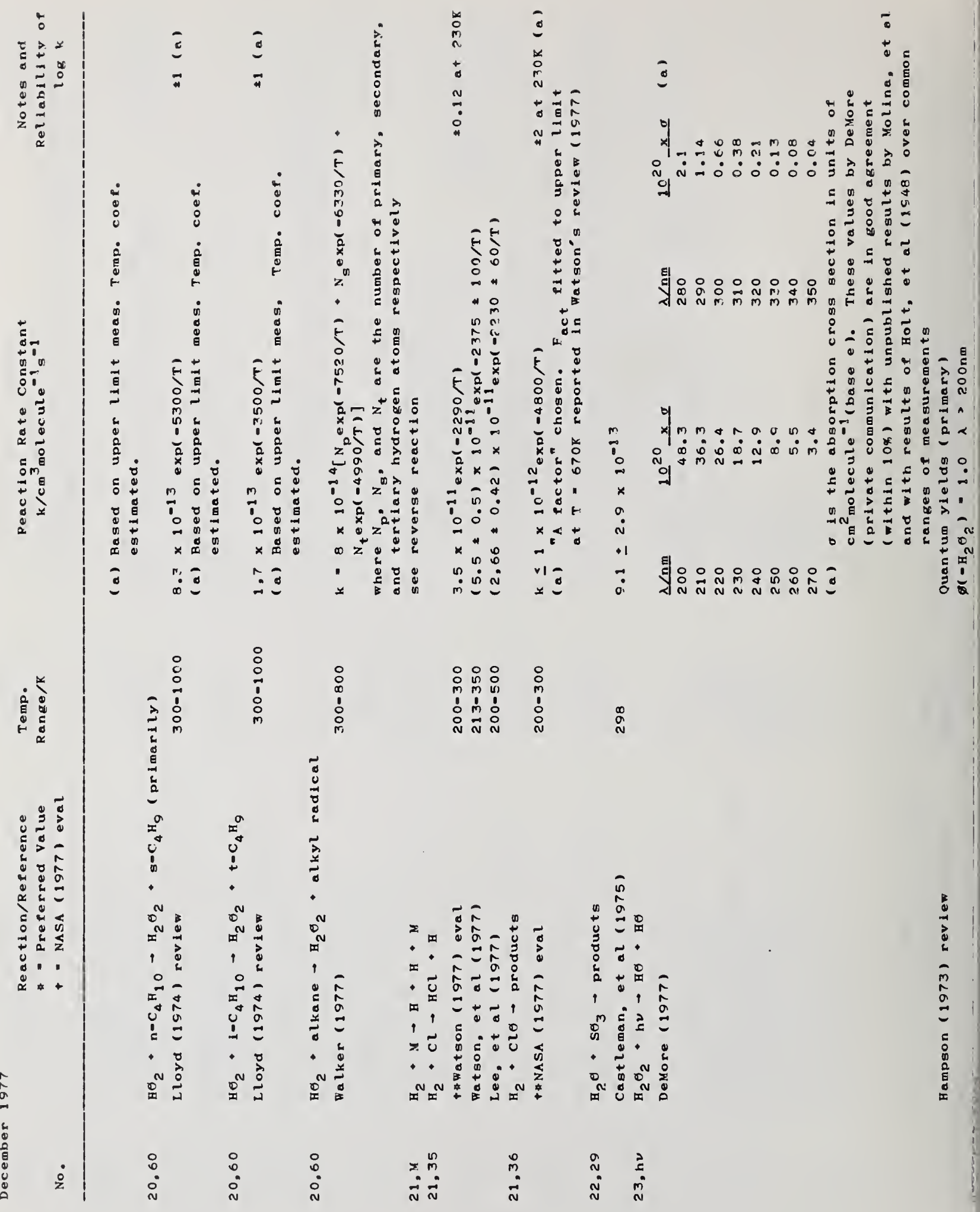




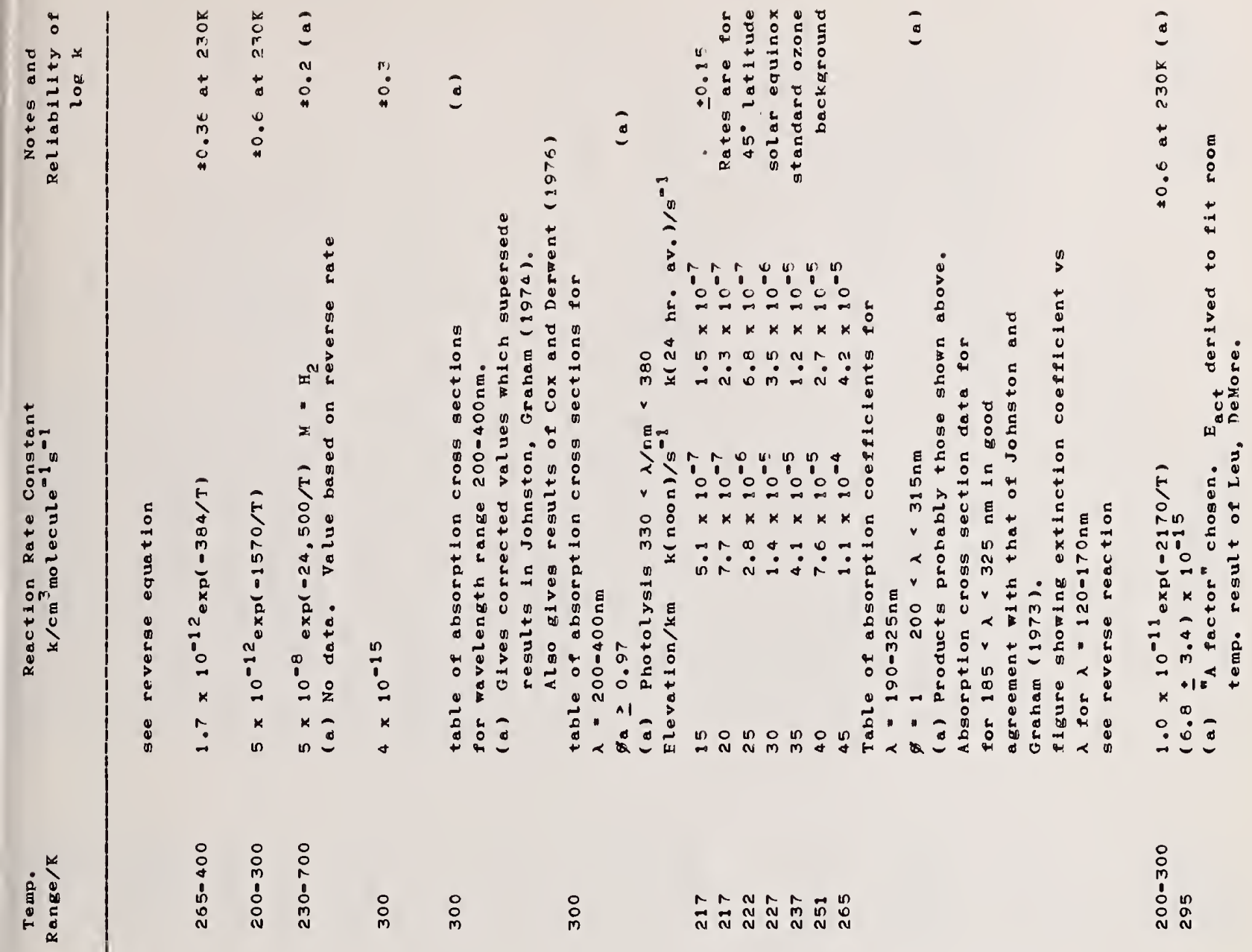

0
$\vdots$
$\vdots$
$\vdots$
$\vdots$
$\vdots$
$\vdots$
$\vdots$

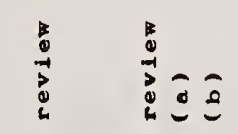

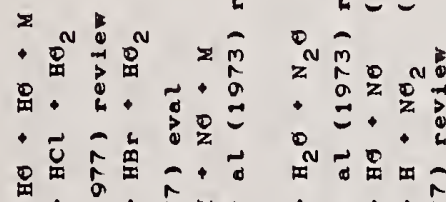

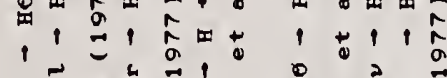

₹ठ

‥

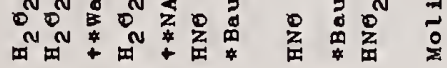

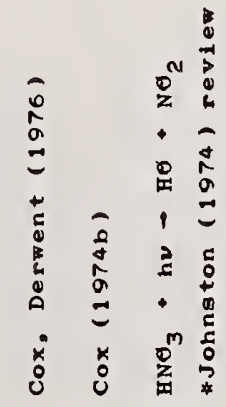

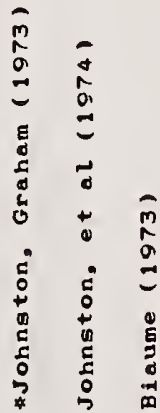

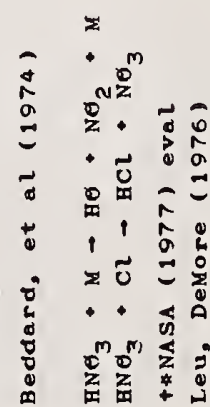

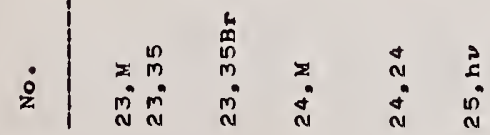

aे

$\stackrel{n}{*}: \stackrel{n}{n}$ 


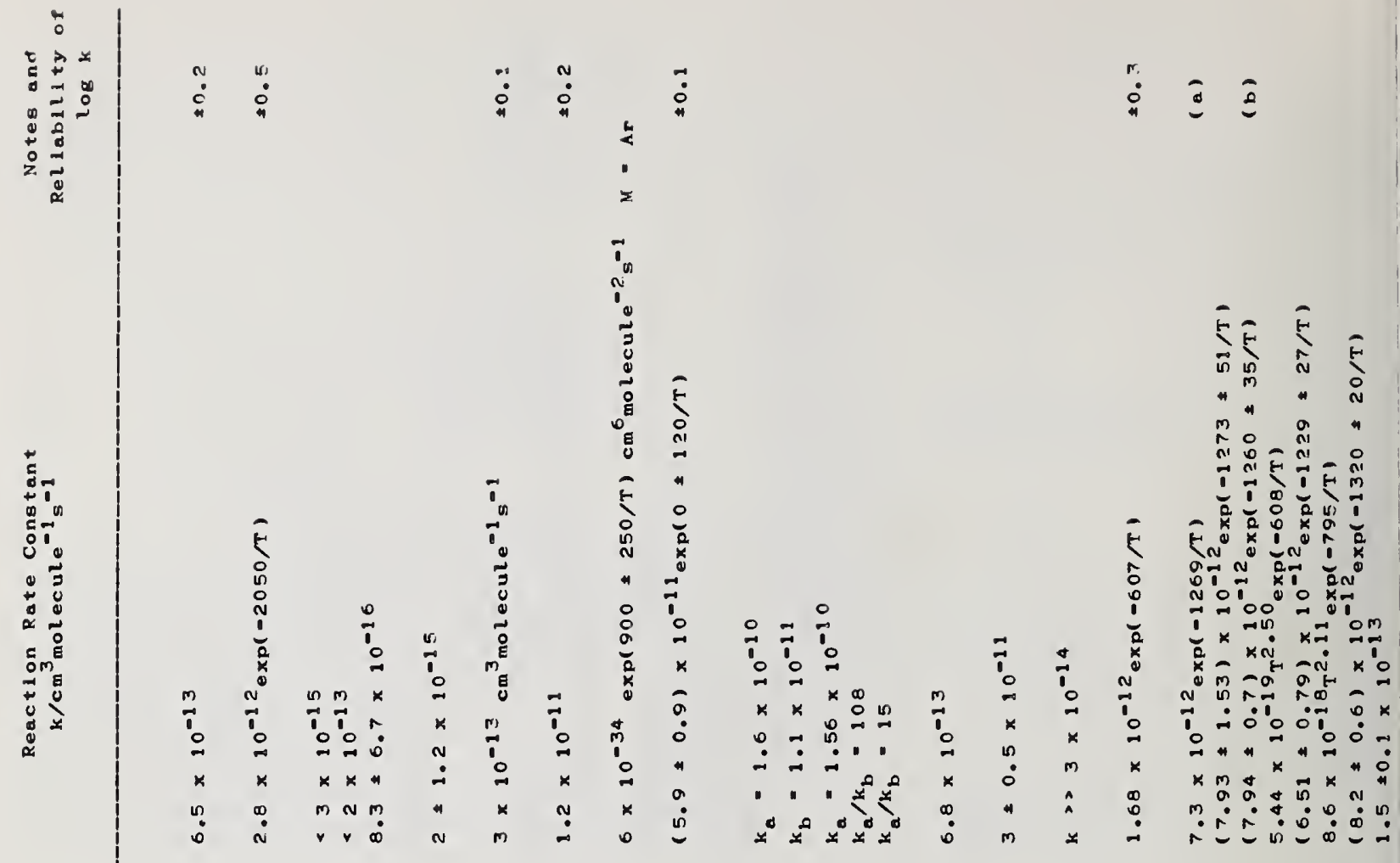

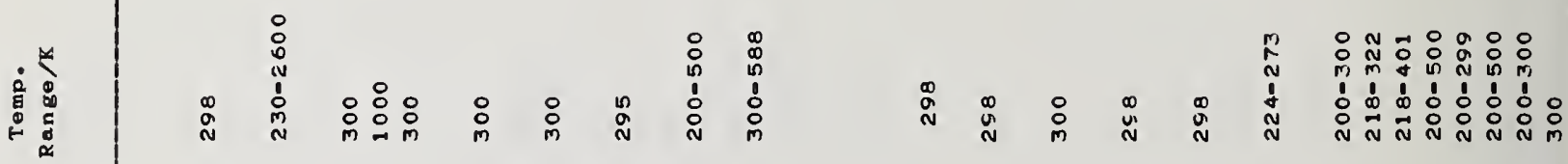

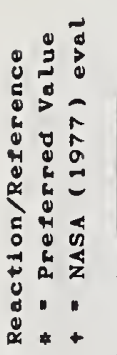

$$
\text { है⿴囗十) }
$$

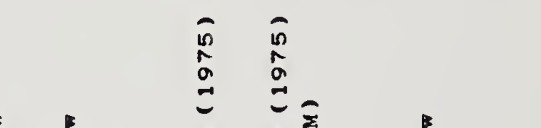

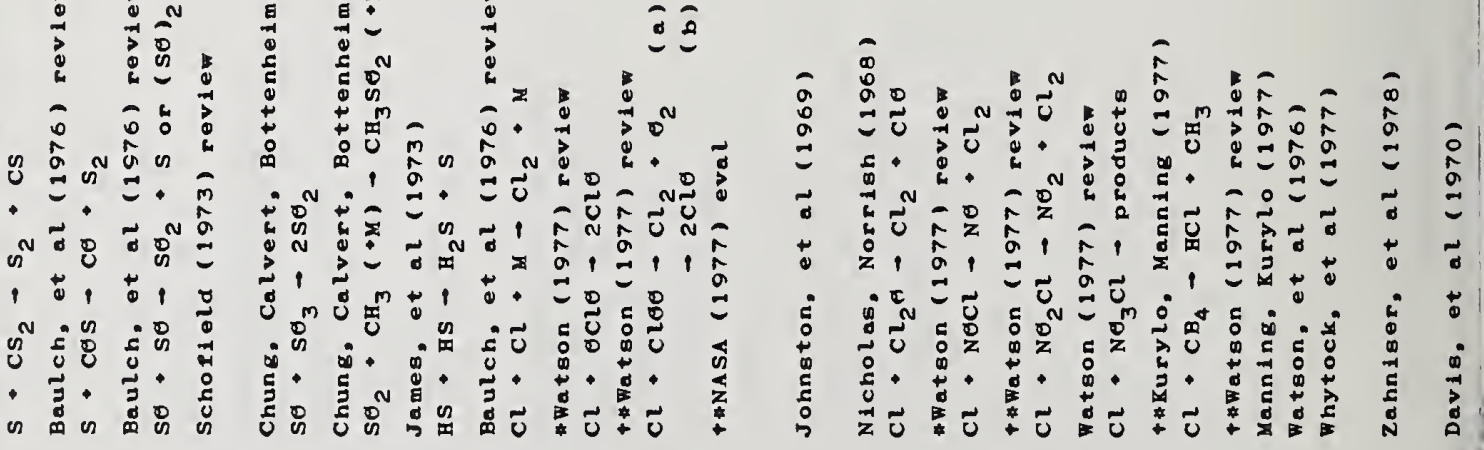

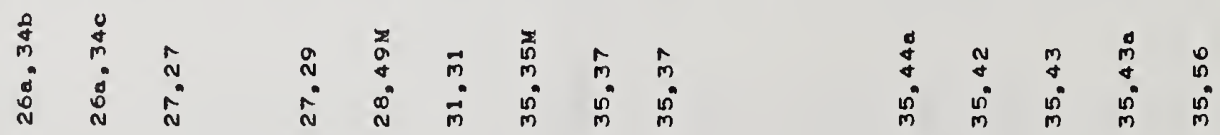




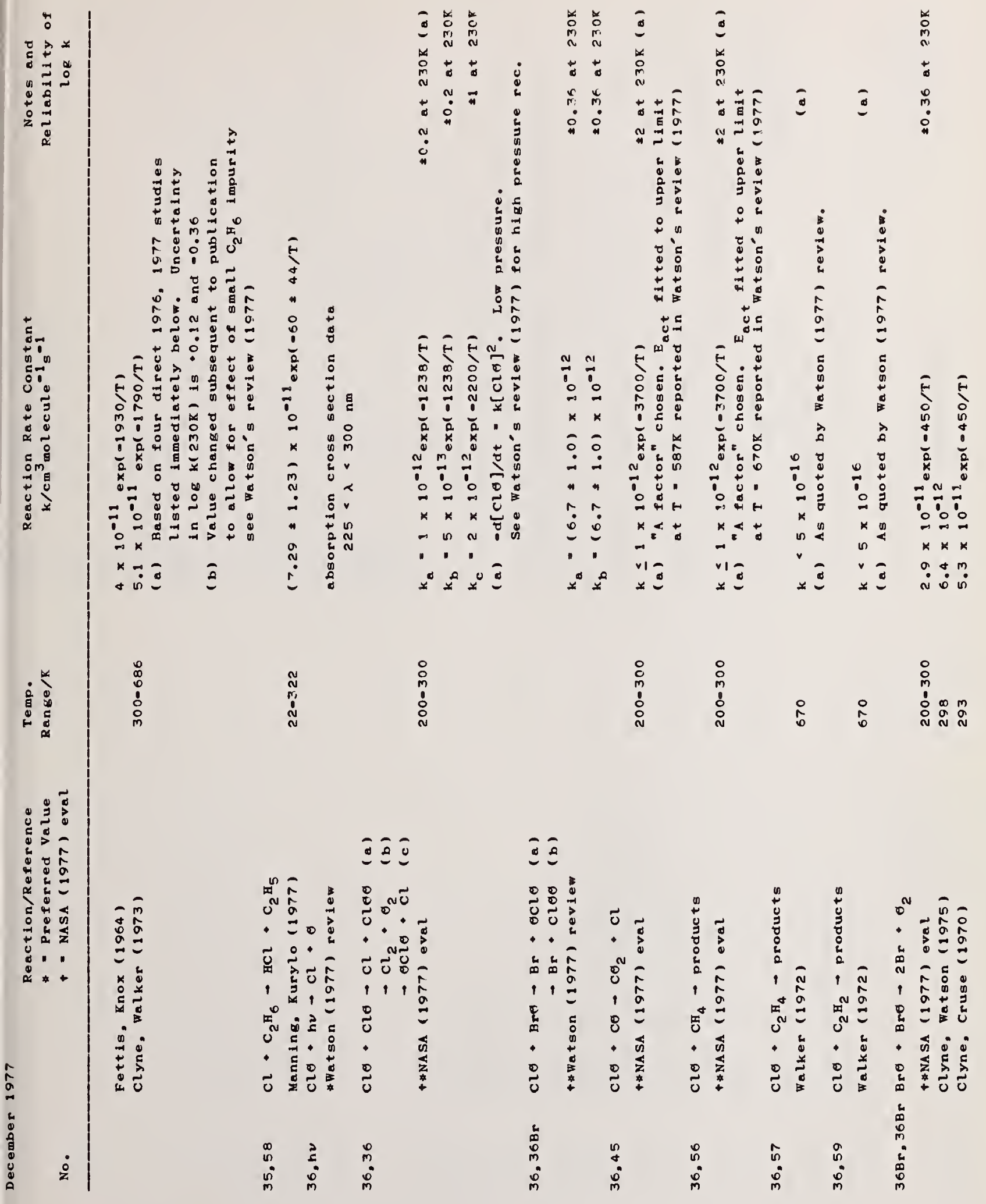




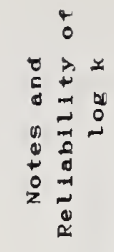

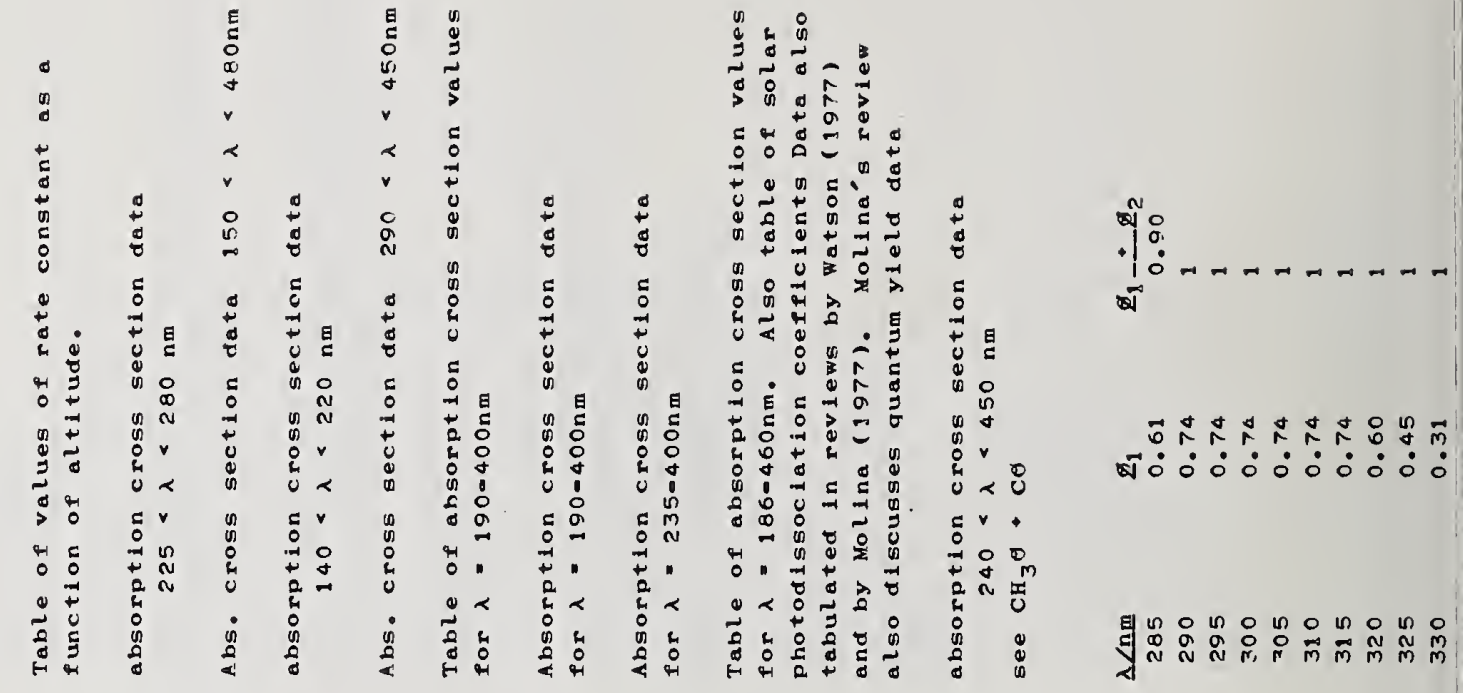

:

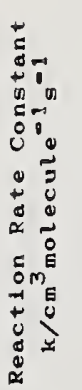

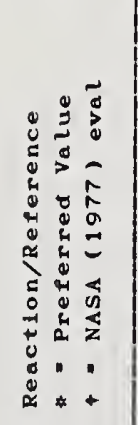

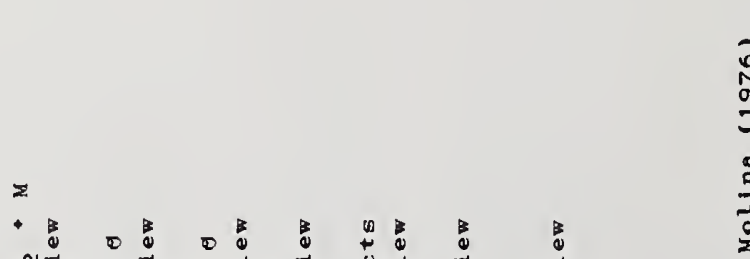

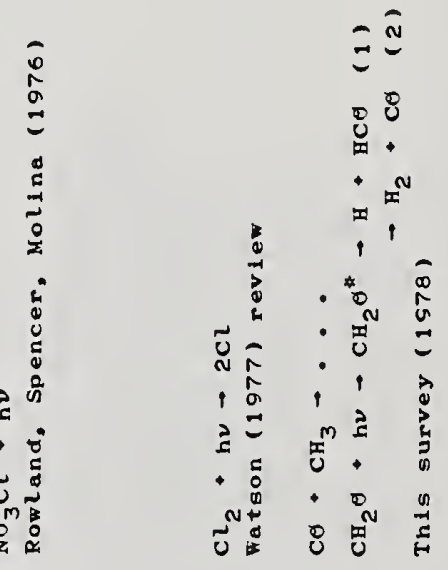

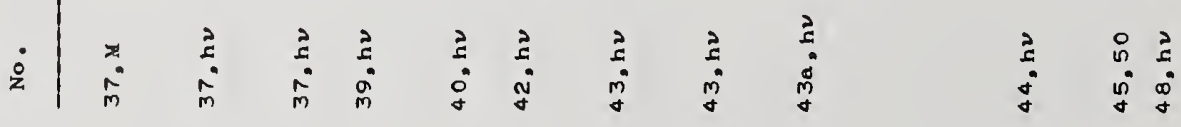



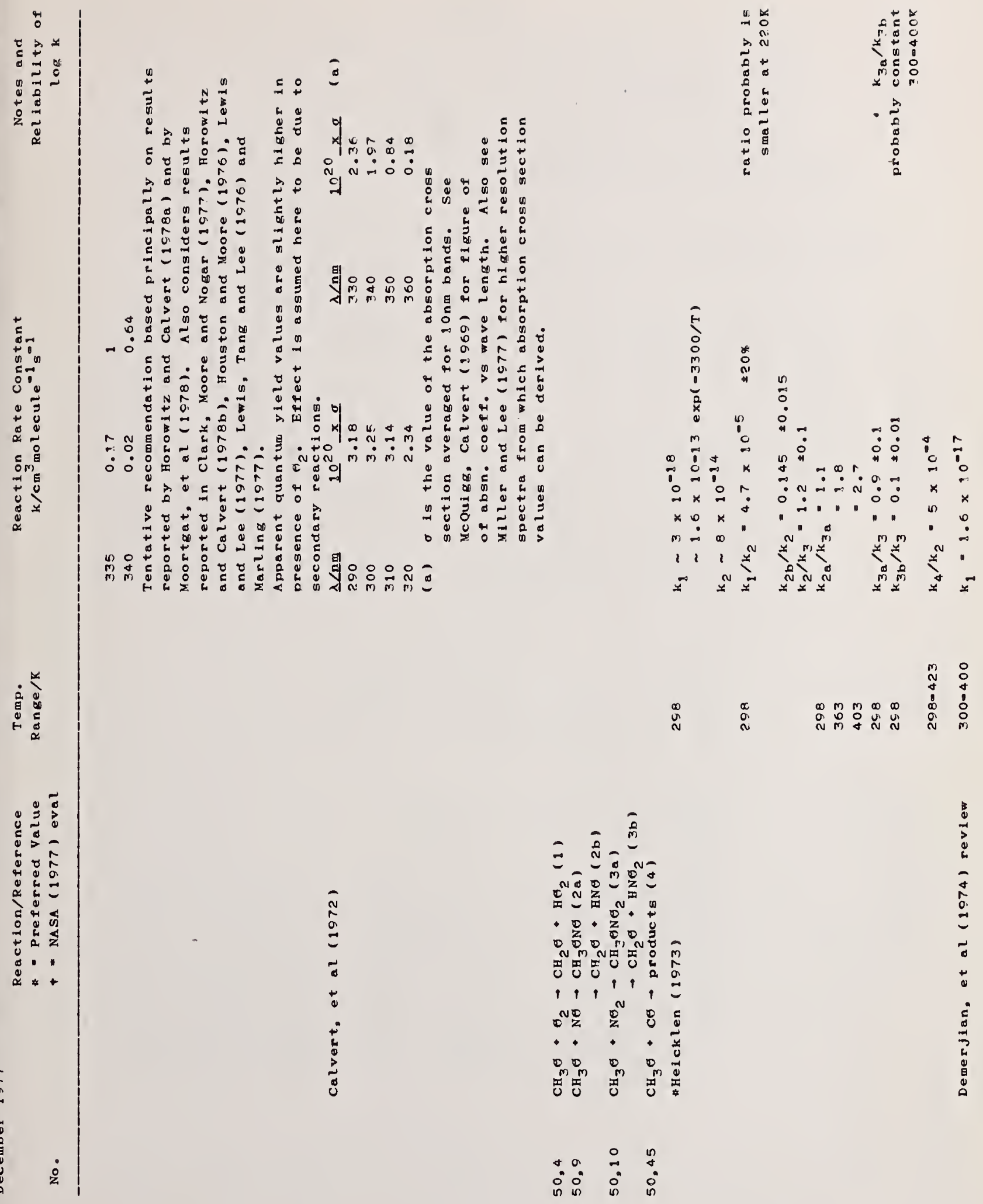


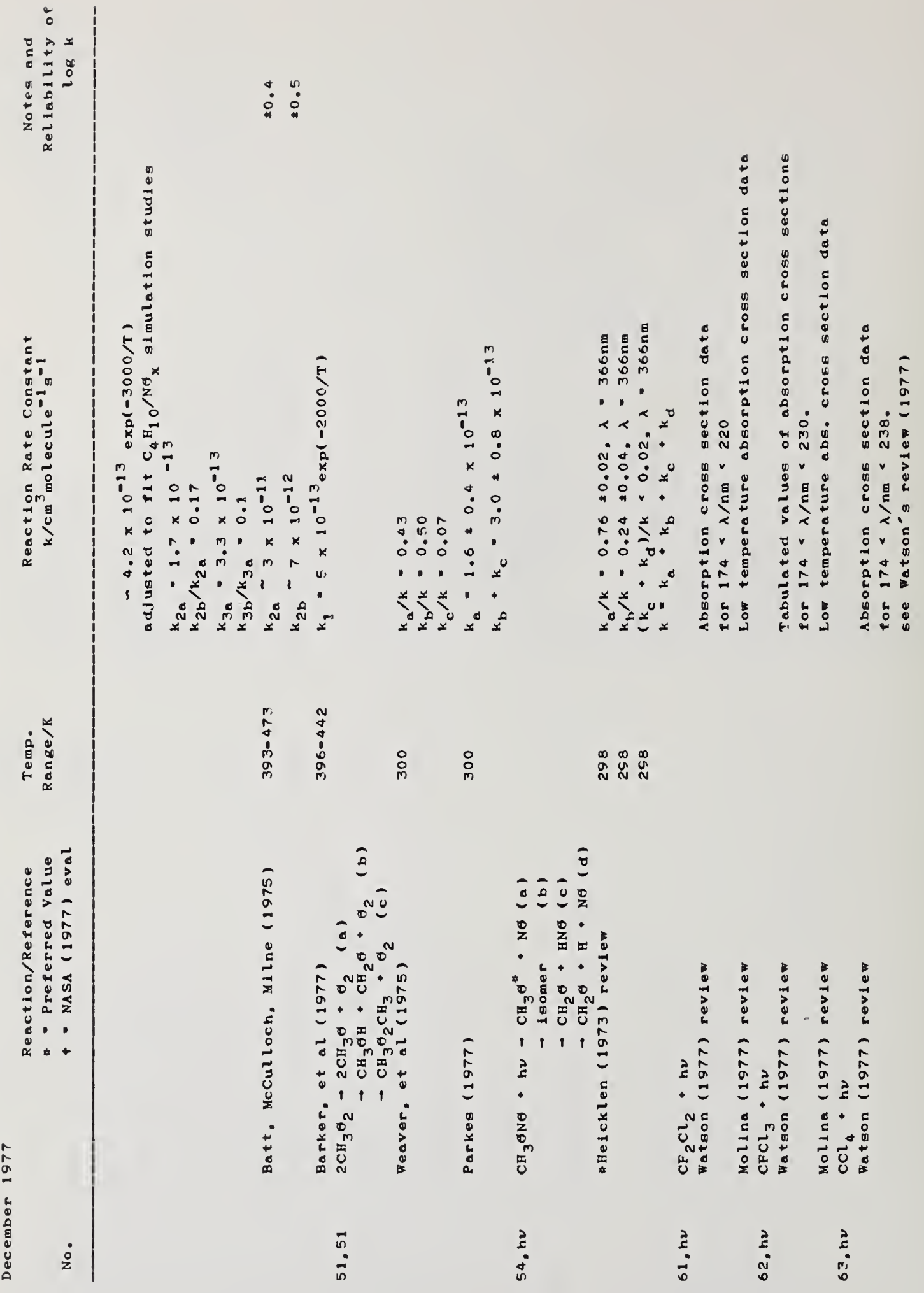




\section{REFERENCES}

Ahumada, J. J., Michael, J. V., and Osborne, D. T.. "Pressure Dependence and Third Body Effects on The Rate Constants for $\mathrm{H} * \theta_{2}, \mathrm{H}$ * No, and $H+C \theta, " J$. Chem. Phys. 57, 3736-3745 (1972)

Albers, E. A., Hoyermann, K., Wagner, H. Gg., and Wolfrum J., "Study of the Reaction of Ammonia with Oxygen Atoms," Symp. Combust. 12th (Combustion Institute, Pittsburgh, 2S69) 313-321

Albers, E. A., Hoyermann, K., Wagner. H. Cg.. and Wolfrum J., "Absolute Measurements of Rate Coefficients for the Reactions of $H$ and $\theta$ Atoms with $\mathrm{H}_{2} \theta_{2}$ and $\mathrm{H}_{2} \theta, "$ Symp. Combust. 13 th (Combustion Institute, Pittsburgh, 1971, 81-88

Albers, E. A., Hoyermann, K., Schacke, H., Schmatjko, K. J., Wagner, H. Gg., and Wolfrum, J., "Absolute Rate Coefficients for the Reaction of H-Atoms with $\mathrm{N}_{2} \theta$ and Some Reactions of CN Radicals," Symp. Combust. i 5 th (Combustion Institute, Pittsburgh, 1975) 765-773

Allen, E. R., and Moortgat, G. K., "Kinetic Studies of the Termolecular Reactions of Hydrogen Atoms with Nitric Oxide and with Molecular Oxygen," Third Int. Symp. Gas Kinetics, Brussels (1573), Abstract DI

Ambidge, P. F., Bradley, J. N., and Whytock, D. A., "Kinetic Study of the Reaction of Hydrogen Atoms with Molecular Chlorine," J. Chem. Soc. Faraday Trans. I 72 1157-1164 (1976)

Ambidge, P. F., Bradley, J. N., and Whytock, D. A., "Kinetic Study of the Reaction of Hydrogen Atoms with Hydrogen Chloride," J. Chem. Soc. Faraday Trans. I 72,214302149 ( $1976 a$ )

Anastasi, C., and Smith, I. W. M., "Rate Measurements of Reactions of $\theta H$ by Resonance Absorption Part 5. Rate Constants for $\theta \mathrm{H}+\mathrm{N \theta}_{2}(+\mathrm{M}) \rightarrow \mathrm{HN}_{3}(+\mathrm{M})$ Gver a Wide Range of Temperature and Pressure," J. Chem. Soc., Faraday Trans. II 72, 1459-1468 (1576)

Anderson, J. G., and Kaufman, F., "Kinetics of the Reaction $\theta H(v=0)+\theta_{Z} \rightarrow$ $\mathrm{H \theta}_{2}+\sigma_{2}, "$ Chem. Phys. Lett. 1S, 483-486 (1973)

Anderson, J. G., Margitan, J. J., and Kaufman, F., "Gas Phase Recombination of $6 \mathrm{H}$ with $\mathrm{N} \theta$ and $\mathrm{NG}_{2}, " \mathrm{~J}$. Chem. Phys, $\underline{60}, 3310-3317$ (1974)

Arin, L. M., and Warneck, P., "Reaction of Gzone with Carbon Monoxide," J. Phys. Chem. 76, 1514-1515 (1972)

Arnoldi, D., and Wolfrum, J., "The Reaction of Vibrationally Excited HCl with Gxygen and Hydrogen Atoms," Chem. Phys. Lett. 24, 234-238 (1974)

Atkinson, R., and Cretanovie, R. J., "Determination of the Rates of Hydrogen Atom Reaction with No by a Modulation Technique," Can. J. Chem. 5i, $370-372$ (1973)

Atkinson, R., Hansen, D. A., and Pitts, J. N., Jr., "Rate Constants for the Reaction of the $\theta \mathrm{H}$ Radical with $\mathrm{H}_{2}$ and $\mathrm{N} \theta\left(\mathrm{M}=\mathrm{Ar}\right.$ and $\left.\mathrm{N}_{2}\right)$, J. Chem. Phys. 62. $3284-3288(1 \subseteq 75 a)$

Atkinson, R., Hansen, D. A., and Pitts, J. N., Jr., "Rate Constants for the Reaction of $\theta \mathrm{H}$ Radicals with $\mathrm{CHF}_{2} \mathrm{Cl}, \mathrm{CF}_{2} \mathrm{Cl}_{2}, \mathrm{CFCl}_{3}$, and $\mathrm{H}_{2}$ over the

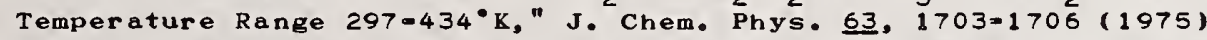

Atkinson, R., Perry, R. A., and Pitts, J. N., Jr., "Kinetics of the Reactions of 6H Radicals with $\mathrm{C \theta}$ and $\mathrm{N}_{2}$ \%, "Chem. Phys. Lett. 44, 204-208 (1976)

Atkinson, R., Perry, R. A., and Pitts, J. N., Jr., "Rate Constants for the Reactions of the $\theta H$ Radical with $N \theta_{2}\left(M=A r\right.$ and $N_{2}$ ) and $S \theta_{2}(M=A r)$," J. Chem. Phys. 65, 306-310 (1976)

Atkinson, R., Perry, R, A, and Pitts, J. N,.,Jr., "Rate Constants for the Reaction of $\theta H$ Radicals with Ethylene Over the Temperature Range 299-425" K," J. Chem. Phys. 66, 1197-1201 (1977) 
Atkinson, R., and Pitts, J. N., Jr., "Temperature Dependence of the Reaction Rate Constants for $\theta\left({ }^{3} \mathrm{P}\right)$ Atoms with $\mathrm{C}_{2} \mathrm{H}_{4}, \mathrm{C}_{3} \mathrm{H}_{6}$ and $N \theta\left(\mathrm{M}=\mathrm{N}_{2}()\right)$, Determined by a Modulation Technique," Chem. Phys. Lett. 27, 467-470 (1974)

Atkinson, R., and Pitts, J. N., Jr., "Rate constants for the Reaction of

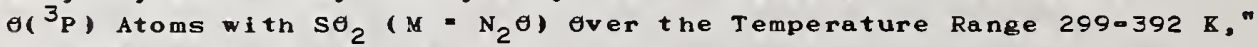
Chem. Phys, Lett, 29, 28-30 (1974)

Atkinson, R., and Pitts, J. N,, Jr., "Temperature Dependence of the Absolute Rate Constants for the Reaction of $\theta\left({ }^{3} P\right)$ Atoms with a Series of Aromatic Hydrocarbons over the Range 299-392* K," J. Phys. Chem. 79, 295-297 (1975a)

Atkinson, R., and Pitts, J. N., Jr., "Rate Constants for the Reaction of $\theta H$ Radicals with Propylene and the Butenes over the Temperature Range 297-425 K," J. Chem. Phys. 63, 3591-3595 (1975b)

Balakhnin, V. P., Egorov, V. I., and Intezarova, E. I., "Kinetics Investigation of the Elemental Reactions of Oxygen Atoms in the Gas Phase by EPR. II. The Reaction $\theta+\mathrm{HCl}=\theta$ H $+\mathrm{Cl}, "$ Kinet. Catal. 12, 258-262 (1971); tr. of: K1net., Katal. 12, 299-303 (1971)

Baldwin, R. R., Gethin, A., and Walker, R. W., "Reaction of Hydrogen Atoms with Nitrous Oxide," J. Chem. Soc., Faraday Trans. I $\underline{\underline{g}}, 352-358$ (1973)

Baldwin, R. R., Fuller, A. R., Longthorn, D., and Palker, R. W., "Addition of Formaldehyde to slowly Reacting Hydrogen * Oxygen Mixtures,"

J. Chem. Soc., Faraday Trans. I $6 \underline{8}, 1362-1367$ ( 1972 )

Baldwin, R. R., Walker, R. W., and Webster, S, J., "The Carbon Monoxideo Sensitized Decomposition of Hydrogen Peroxide," Combust. Flame 15. $167-172$ ( 1970$)$

Ball, M. J., and Larkin, G. S., "Determination of the Rates of Atomic Reactions by the Discharge-Flow Method," Nature Phys. Sci. 245, 63-64 (1973)

Bar-Ziv, E., Moy, J., and Gordon, R. J., "Temperature Dependence of the Laser Enhanced Reaction $\mathrm{N \theta}+\theta_{3}(001)$ II. Contribution from Reactive and Non-Reactive Channels," J. Chem. Phys. 68, $1013(1978)$

Barassin, J., and Combourieu, J., "No. 1 - Etude Cinetique des Reactions entre l-Oxygene Atomique et less Derives Chlores du Methane. II. - Reactions $\mathrm{CH}_{3} \mathrm{Cl}+\theta, \mathrm{CHCl}_{3}+\theta, \mathrm{CCl}_{4}+\theta$ et $\mathrm{CH}_{4}+\theta$ : Resultats Experimentaux, Bull. Soc. Chim. Fr. 1-5 (1974)

Barker, J. R., Benson, S, W., and Golden, D, M., "The Decomposition of Dimethyl Peroxide and the Rate Constant for $\mathrm{CH}_{3} \theta+\theta_{2} \rightarrow \mathrm{CH}_{2} \theta^{*} \mathrm{H \theta}_{2}$," Int. J. Chem. Kinet. 9, 31-53 (1977)

Basco, N., James, D. G. L., and James, F. C., "A Quantitative Study of Alkyl Radical Reactions by Kinetic Spectroscopy. II. Combination of the Methyl Radical with the Oxygen Molecule," Int. J. Chem. Kinet. $4,129-149$ (1972)

Basco, N., James, D. G. L., and Suart, R. D., "A Quantitative Study of Alkyl Radical Reactions by Kinetic Spectroscopy. Part I. Mutual Combination of Methyl Radicals and Combination of Methyl Radicals with Nitric Oxide," Int. J. Chem. Kinet. 2, 215-234 (1970)

Bass, A. M., and Laufer, A. H., "The Methyl Radical Combination Rate Constant as Determined by Kinetic Spectroscopy," Int. J. Chem. Kinet. 5, 10531065 (1973)

Bass, A. M., Ledford, A. E, Jr., and Laufer, A. H,, "Extinction Coefficients of $\mathrm{NO}_{2}$ and $\mathrm{N}_{2} \mathrm{O}_{4}$, J. Res. Nat. Bur. Stand., Sect. $A \underline{80}$. $143-166$ (1976) 
Batt, L., and McCulloch, R. D., "Pyrolysis of Dimethyl Peroxide," Int. J. Chem. Kinet. $8,491-500(1976)$

Batt, L., McCulloch, R. D., and Milne, R. T., "Thermochemical and Kinetic Studies of Alkyl Nitrites (RONO) - $D(R \theta-N g)$, The Reactions between Rब* and $N \theta$, and the Decomposition of $R \theta^{*}, "$ Int. J. Chem. Kinet., Symp. No. 1, $441-461(1975)$

Baulch, D. L., and Drysdale, D. D., "An Evaluation of the Rate Data for the Reaction $\mathrm{CO} \cdot \theta \mathrm{H} \rightarrow \mathrm{C \theta}_{2}+\mathrm{H}$, "Combust. Flame $23,215-225$ ( 1974 )

Baulch, D. L., Drysdale, D. D., Horne, D. G., and Lloyd, A. C., "Evaluated Kinetic Data for High Temperature Reactions, Vol. 1 : Homogeneous Gas Phase Reactions of the $\mathrm{H}_{2}-\mathrm{\theta}_{2}$ System," (Butterworths, London, 1972 )

Baulch, D. L., Drysdale, D. D., and Horne, D. G., "Evaluated Kinetic Data for High Temperature Reactions, Vol. 2: Homogeneous Gas Phase Reactions of the $\mathrm{H}_{2}-\mathrm{N}_{2}-\mathrm{O}_{2}$ System," (Butterworths, London, 1973) Baulch, D. L., Drysdale, D. D., Duxbury, J., and Grant, S. J., "Evaluated Kinetic Data for High Temperature Reactions, Vol. 3: Homogeneous Gas Phase Reactions of the $\theta_{2}-\theta_{3}$ System, the c $\theta_{-} \theta_{2}-\mathrm{H}_{2}$ System, and of Sulphur-Containing Species," (Butterworths, London, 1976)

Baulch, D. L., Drysdale, D. D., and Horne, D. G., [unpublished data sheets prepared for this survey] University of Leeds, 1973

Baulch, D. L., Drysdale, D. D., and Horne, D. G.. "Rate Constants for Reactions of the $\mathrm{H}_{2}-\mathrm{N}_{2}-\theta_{2}$ System," in Chemical Kinetics Data Survey $v$ Sixty-six Contributed Rate and Photochemical Data Evaluations on Ninety-four Reactions, NBSIR 73-206, D. Garvin, Ed., National Bureau of Standards, Washington, D. C. ( 1973 ) pages 49-115

Bayes, K. D., letter to RFH, March 11, 1974

Becker, K. H., Groth, W., and Kley, D., "The Rate Constant of the Aeronomic Reaction $\mathrm{N} \cdot \theta_{2}, " \mathrm{Z}$. Naturforsch. A 24 , 1280-1281 (1969)

Becker, K. H., Groth, W., and Schurath, U., "The Quenching of Metastable $\theta_{2}\left({ }^{1} \Delta_{g}\right)$ and $\theta_{2}\left({ }^{1} \Sigma_{g}\right)$ Molecules," Chem. Phys. Lett. $\underline{8}, 259-262$ ( 1971 )

Becker, K. H., Groth, W., and Schurath, U., "Reactions of $\theta_{2}\left({ }^{1} \Delta_{g}\right.$ with Gzone," Chem. Phys. Letts. 14, 4890492 (1972)

Becker, K. H., Groth, W., and Thran, D., "Mechanism of the Air Afterglow $\mathrm{NO} \cdot \sigma \rightarrow \mathrm{NO}_{2}$ - hu," Symp. Combust. 14 th (Combustion Institute, Pittsburgh, 1973 ) 353-363

Becker, K. H., Inocencio, M., and Schurath, U., "The Reaction of Ozone with Hydrogen Sulfide and its Organic Derivatives," Int. J. Chem. Kinet., Symp. No. 1, 205-220 (1975)

Becker, K. H., Schurath, U,, and Seitz, H,, "Ozone-olefin Reactions in the Gas Phase. 1. Rate Constants and Activation Energies," Int. J. Chem. Kinet. $\underline{6}$, $725-739(1974)$

Beddard, G. S., Giachardi, D. J., and Wayne, R. P., "The Vacuum Ultra-violet Absorption Spectrum of Anhydrous Nitric Acid," J. Photochem. $3,321-323$ $(1974 / 75)$

Bedford, G., and Thomas, J, H., "Reaction between Ammonia and Nitrogen Dioxide," J. Chem. Soc., Faraday Trans. I 68, 2163-2170 (1972)

Bemand, P. P., and Clyne, M. A. A., "Atomic Resonance Fluorescence for Rate Constants of Rapid Bimolecular Reactions. Part 6.-Hydrogen Atom Reactions: $\mathrm{H}$ - $\mathrm{Cl}_{2}$ from 300 to $730 \mathrm{~K}$ and $\mathrm{H}+\mathrm{NO}_{2}$ at $298 \mathrm{~K}$," J. Chem. Soc., Faraday Trans. I I 73. 394-405 (1977)

Bemand, P. P., Clyne, M. A. A,, and Watson, R. T., "Reactions of Chlorine Oxide Radicals. Part 4.-Rate Constants for the Reaction Cl OClO, 


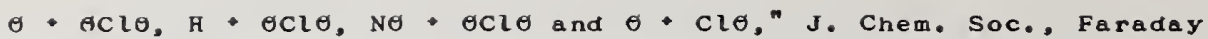
Trans. I $\underline{69}, 1356-1374$ (1973)

Bemand, P. P., Clyne, M. A. A., and Watson, R. T., "Atomic Resonance Fluorescence and Mass Spectrometry for Measurements of the Rate Constants for Elementary Reactions: $\theta\left({ }^{3} \mathrm{P}_{\mathrm{J}}\right)+\mathrm{N}_{2} \rightarrow \mathrm{N} \theta_{2} \cdot \theta_{2}$ and $\mathrm{NO}+\theta_{3} \rightarrow \mathrm{N \theta}_{2} \rightarrow \theta_{2}, " \mathrm{~J}$. Chem. Soc., Faraday Trans. II 70, 564-576 (1974) Benson, S. W., and $\theta$ "Neal, H. E., "Kinetic Data on Gas Phase Unimolecular Reactions," NBS-NSRDS-21 (1970). (Supt. Doc., U.S. Govt. Printing Office, Washington, D.C. 20402)

Bethke, G. W.. "Oscillator Strengths in the Far Ultraviolet. I. Nitric Oxide," J. Chem. Phys. 31, 662-668 (1559)

Bevan, P. L., and Johnson, G. R. A., "Kinetics of Gzone Formation in the Pulse Radiolysis of Oxygen Gas," J. Chem. Soc., Faraday Trans. I 69, $216-227(1973)$

Biaume, F., "Nitric Acid Vapour Absorption Cross-Section Spectrum and Its Photodissociation in the Stratosphere," J. Photochem. 2, 139-140 (1973)

Bida, G. T., Rreckenridge, W. H., and Kolln, w. S., "A Kinetic Study of the Very Fast Reaction: $\theta\left({ }^{3} \mathrm{P}\right) \cdot \mathrm{CS} \rightarrow \mathrm{C}^{+} \cdot \mathrm{S}\left({ }^{3} \mathrm{P}\right), " \mathrm{~J}$. Chem. Phys. 64. $3296-3302(1976)$

Biermann, H. W., Zetzsch, C., and Stuhl, F., "Rate Constant for the Reaction of $6 \mathrm{H}$ with $\mathrm{N}_{2} \theta$ at $298 \mathrm{~K}$," Ber. Bunsenees. Phys. Chem. 80, 909-911 (1976)

Biermann, H. W., Zetzsch, C., and Stuhl, F., "On the Pressure Dependence of the Reaction of HO with co," paper presented at 13 th Informal Conference on Photochemistry, Clearwater Beach, Florida, Jan. 1978

Bingham, F. W., Johnson, A. W., and Rice, J. K., "Rate Coefficient for Deactivation of $\theta\left({ }^{1} S\right)$ by $H_{2}{ }^{A}, " J$. Chem. Phys. 65, 1663-1665 (1976)

Biordi, J. C., Lazzara, C. P., and Papp, J. F., "Flame Structure Studies of $\mathrm{CF}_{3} \mathrm{Br}-\mathrm{Inhibited}$ Methane Flames. II. Kinetics and Mechanisms," Symp. Combust. 15th (Combustion Institute, Pittsburgh, 1975) 917-932

Birks, J. W., Shoemaker, B., Leck, T. J., and Binton, D. Y., "Studies of Reactions of Importance in the Stratosphere I. Reaction of Nitric $\theta x i d e$ with Ozone," J. Chem. Phys. 65, 5181-5185 (1976)

Birks, J. W., Shoemaker, B., Leck, T, J., Borders, R. A., and Hart, L. J., "Studies of Reactions of Importance in the Stratosphere. II. Reactions Involving Chlorine Nitrate and Chlorine Dioxide,"J. Chem. Phys. 66, $4591-4599(1977)$

Bishop, W. P., and Dorfman, I. M., "Pulse Radiolysis Studies. XVI. Kinetics of the Reaction of Gaseous Hydrogen Atoms with Molecular Gxygen by Fast Iyman- $\alpha$ Absorption Spectrophotometry, "J. Chem. Phys. 52, 3210-3216 (1970)

Blume, C. W., Hisatsune, I. C., and Helcklen, J., "GasePhase Gzonolysis of Cigo and Trans-Dichloroethylene," Int.J. Chem. Kinet. $8,235-258$ (1976)

Bowman, C. T., "Non-Equilibrium Radical Concentrations in Shock-Initiated Methane Oxidation," Symp. Combust. 15th (Combustion Institute, Pittsburgh, 1975 ) 8690882

Bradley, J. N., Hack, W., Hoyermann, K., and Wagner, H. Gg., "Kinetics of the Reaction of Hydroxyl Radicals with Ethylene and with $\mathrm{C}_{3}$ Hydrocarbons," J. Chem. Soc., Faraday Trans. I 69, 188901898 (1973)

Braslarsky, S., and Feicklen, J., "The Gas-Phase Reaction of $\sigma_{3} w_{i t h} H_{2} c \theta_{,}$ Int. J. Chem. Kinet. $8,801-808$ (1976)

Breckenridge, W. H., and Miller, T. A., "Kinetic Study by EPR of the Production and Decay of $\left.\operatorname{se}^{1} \Delta\right)$ in the Reaction of $\theta_{2}\left({ }^{1} \Delta_{g}\right)$ with $\operatorname{se}\left({ }^{3} \Sigma^{-}\right), " J$. Chem. Phys, 56, 465-474 (1972) 
Brown, R, D. H., Glass, G. P., and Smith, I. W. M., "The Relaxation of HCl( $v=1)$ and DCL $(v=1)$ by $\theta$ Atoms Between 196 and $400 \mathrm{~K}$, "Chem. Phys. Lett. 32, $517-520(1975)$

Brown, R. D. H., and Smith, I. W. M., "Absolute Rate Constants for the Reactions $\left({ }^{3} P\right)$ Atoms with HCl and HBr, "Int. J. Chem. Kinet . $301-315(1575)$

Burrows, J. P., Harris, G. W., and Thrush, B. A., "Rates of Reaction of $H \theta_{2}$ with $H \theta$ and $\theta$ studied by Laser Magnetic Resonance," Nature 267, 233-234 (1977)

Butler, R., Solomon, I. J., and Snelson, A., "Pressure Dependence of the co - GH Rate Constant in $\sigma_{2}+\mathrm{N}_{2}$ Mixtures," Chem. Phys. Lett., in press (1977)

Calvert, J. G., Kerr, J. A., Demerjian, K. L., and McQuige, R. D., "Photolysis of Formaldehyde as a Hydrogen Atom Source in the Lower Atmosphere," science 175, 751-752 (1972)

Campbell, I. M.. and Goodman, K., "Reaction of $\theta\left({ }^{3} P\right.$ ) Atoms with Nitromethane Vapour at $295 \mathrm{~K}$, " Chem. Phys. Lett. 34 , 105-108 (1975a)

Campbell, I. M., and Goodman, K., "Rate Constants for Reactions of Hydroxyl Radicals with Nitromethane and Methyl Nitrite Vapours at $292 \mathrm{~K}$, Chem. Phys. Lett. $36,382-384$ (1975b)

Campbell, I. M., and Gray, C. N., "Rate Constants for $\theta\left({ }^{3} P\right)$ Recombination and Association with N( ${ }^{4}$ S)," Chem. Phys. Lett, 18, 607-609 (1973)

Campbell, I. M., and Handy, B. J., "Studies of Reactions of Atoms in a Discharge Flow stirred Reactor Part 1 - The $\theta+\mathrm{H}_{2}$ - NO System," J. Chem. Soc., Faraday Trans. I 71, 2097-2106 (1975)

Campbell, I. M., Mclaughlin, D. F., and Handy, B. J., "Rate Constans for Reactions of Hydroxyl Radicals with Alcohol Vapours at $292 \mathrm{~K}$, "Chem. Phys. Lett. 3ㅗㅛ, 362-364 (1976)

Castellano, E., and Schumacher, H. J., "La Descomposicion Fotoquimica del Ozono a $3200 \mathrm{~A}, "$ An. Asoc. Quim. Argent. 63.,9-15 (1975)

Castleman, A. W., Jr., Davis, R. E., Munkelwitz, H. R., Tang, I. N., and Wood, W. Po, "Kinetics or Association Reactions Pertaining to $\mathrm{H}_{2} \mathrm{S \theta}_{4}$ Aerosol Formation," Int. J. Chem. Kinet., Symp. No. 1, 629-640 (1975)

Castleman, A. W.. Jr., and Tang, I. N., "Kinetics of the Association Reaction of $\mathrm{S \sigma}_{2}$ with the Hydroxyl Radical," J. Photochem. 6, 349-354 (1977)

Chan, W. H., Nordstrom, R. J., Calvert, J. G., and Shaw, J. H., "Kinetic Study of HoN Formation and Decay Reactions in Gaseous Mixtures of HøNG, $\mathrm{N} \theta, \mathrm{NO}_{2}, \mathrm{H}_{2} \sigma$, and $\mathrm{N}_{2}$," Environ. Sci. Technol, 10, 674-682 (1976); see also article by same authors (which is next entry), Chem. Phys. Lett. 37, $441-446$ (1976)

Chan, W. H., Nordstrom, R. J., Calvert, J. G., and Shaw, J. H., "An IRFTS Spectroscopic study of the kinetics and the Mechanism of the Reactions in the Gaseous System, HबNO, NO, Nब, $\mathrm{H}_{2}, "$ Chem. Phys, Lett. 37 , $441-446(1976)$

Chan, W. H., Uselman, W. M., Calvert, J, G., and Shaw, J. H, "The Pressure Dependence of the Rate Constant for the Reaction: H $+\mathrm{C \theta} \rightarrow \mathrm{H}+\mathrm{C} \theta_{2}, "$ Chem. Phys. Lett. 45, 240-244 (1977)

Chang, J. S., and Kaufman, F., "Upper Limits of the Rate constants for the Reactions of $\mathrm{CFCl}_{3}(\mathrm{~F}-11), \mathrm{CF}_{2} \mathrm{Cl}_{2}(\mathrm{~F}-12)$, and $\mathrm{N}_{2} \theta$ with $\theta \mathrm{H}$ and Estimates of Corresponding Lower Limit of their Tropospheric Lifetimes," Geophys. Res. Lett. $4,192-154$ (1977) 
Chang, J. S., and Kaufman, F., "Kinetics of the Reactions or Hydroxyl Radicals with Some Halocarbons: $\mathrm{CHFCl}_{2}, \mathrm{CHF}_{2} \mathrm{Cl}, \mathrm{CH}_{3} \mathrm{CCl}_{3}, \mathrm{C}_{2} \mathrm{HCl}_{3}$, and $\mathrm{C}_{2} \mathrm{Cl}_{4}$,"

J. Chem. Phys. 66, 4989-4994 (1977)

Chapman, C. J., and Wayne, R. P., "The Reaction of Atomic Oxygen and Hydrogen with Nitric Acid," Int. J, Chemie Kinet. 6, 617-630 (1974)

Chung, K., Calvert, J. G., and Bottenheim, J. W.. "The Photochemistry of Sulfur Dioxide Excited within its First Allowed Band (3130 o) and the 'Forbidden' Band (3700-4000 o)," Int. J. Chem. Kinet. I, 161-182 (1975)

Clark, I. D., Jones, I. T. N, and Wayne, R. P.., "The Kinetics of the Reaction between $\theta_{2}\left({ }^{1} \Delta_{g}\right)$ and Ozone," Proc. Roy. Soc. London A 3217, 407-416 (1970)

Clark, J. H., Moore, C. B., and Nogar, N. S., "The Photochemistry of Formaldenyde: Absolute Quantum Yields, Radical Reactions and No Reactions," typescript, Dept. of Chemistry, Univ. of California, Berkeley, CA (1977)

Clark, I, D., and Wayne, R. P., "Kinetics of the Reaction between Atomic Nitrogen and Molecular oxygen in the Ground ( ${ }^{3} \Sigma_{g}$, and First Excited ( $\left.{ }^{1} \Delta_{E}\right)$ states," Proc. Roy. Soc. London A 316, 539-550 (1970)

clough, P. N., and Thrush, B. A., "Mechanism of Chemiluminescent Reaction between Nitric Oxide and Ozone," Trans. Faraday Soc. 63. $915=925$ (1967)

Clyne, M. A. A., and Coxon, J. A., "Kinetics studies of Exyhalogen Radical Systems," Proc. Roy. Soc., (London) A $\underline{303}, 207-231$ (1968)

Clyne, M. A. A., and Cruse, H. W.. "Studies of Ground-State ${ }^{2} P_{3 / 2}$ Halogen Atoms Using Atomic Resonance Absorption," Trans. Faraday Soc. 67. $2869-2885$ (1971)

clyne, M. A. A., and Cruse, H. W., "Atomic Resonance Fluorescence Spectrometry for Rate Constants of Rapid Bimolecular Reactions. Part 1. Reactions O $\mathrm{NO}_{2}, \mathrm{Cl} \cdot \mathrm{ClN} \theta, \mathrm{Br}+\mathrm{ClN}, " \mathrm{~J}$. Chem. Soc., Faraday Trans. II 68, 12810 1259 ( 1972 )

clyne, M. A. A., and Cruse, H. W.. "Rates of Elementary Reaction Involving the $\operatorname{Br} \theta\left(X^{2} \Pi\right)$ and $I \theta\left(X^{2} \Pi\right)$ Radicals. Part 1.-Formation and Decay of the Bro Radical," Trans. Faraday Soc. 66, 2214-2226 (1970)

clyne, M. A. A., and Down, S., "Kinetic Behaviour of $\theta H X^{2} \Pi$ and $A^{2} \Sigma^{*}$ using Molecular Resonance Fluorescence Spectrometry," J. Chem. Soc. Faraday Trans. II 70, 253-266 (1974)

Clyne, M. A. A., and McDermid, I. S., "Mass Spectrometric Determinations of Rates of Elementary Reactions of $\mathrm{N} \theta$ and of $\mathrm{NO}_{2}$ with Ground State $\mathrm{N}^{4} \mathrm{~S}$ Atoms," J. Chem. Soc,. Faraday Trans. I I1, $2189-2208$ (1575)

Clyne, M. A. A., and Monkhouse, P. B., "Atomic Resonance Fluorescence for Rate Constants of Rapid Bimolecular Reactions. Part 5-Hydrogen Atom Reactions; $\mathrm{H} \cdot \mathrm{NO}_{2}$ and $\mathrm{H} \cdot \theta_{3}, " \mathrm{~J}$. Chem. Soc., Faraday Trans. II 73 , 298-309 ( 1977 )

Clyne, M. A. A., Monkhouse, P. B., and Townsend, L. W., "Reactions of $\theta{ }^{3}$, Atoms with Halogens: The Rate Constants for the Elementary Reactions $\theta+\mathrm{BrCl}, \theta+\mathrm{Br}_{2}$, and $\theta \cdot \mathrm{Cl}_{2}$ " Int. J. Chem. Kinet. $8,425-449$ (1976)

Clyne, M. A. A., and Nip, W. S., "Reactions of Chlorine oxide Radicals. Part 6.-The Reaction $\theta+\mathrm{Cl} \theta \rightarrow \mathrm{Cl}+\theta_{2}$ from 220 to $425 \mathrm{~K}$, "J. Chem. Soc., Faraday Trans. I 72, 2211-2217 (1976)

Clyne, M. A. A., and Nip, w. S., "Study of Elementary Reactions by Atomic Resonance Absorption with a Non-Reversed Source Part 1 - The Reaction $\mathrm{Cl}+\theta_{3} \rightarrow \mathrm{Cl} \theta+\theta_{2}, " \mathrm{~J}$. Chem. Soc., Faraday Trans. II $Z 2$, $838-847(1976)$ 
Clyne, M. A. A., and Walker, R. F., "Absolute Rate Constants for Elementary Reactions in the Chlorination of $\mathrm{CH}_{4}, \mathrm{CD}_{4}, \mathrm{CH}_{3} \mathrm{Cl}, \mathrm{CH}_{2} \mathrm{Cl}_{2}, \mathrm{CHCl}_{3}, \mathrm{CDCl}_{3}$ and $\mathrm{CBrCl}_{3}$," J. Chem. Soc., Faraday Trans. I 69, 1547-1567 (1973)

Clyne, M. A. A., and Watson, R. T., "Kinetics Studies for Diatomic Free Radicals using Mass Spectrometry Part 3 - Elementary Reactions Involving Bro $x^{2} \Pi$ Radicals," J. Chem. Soc., Faraday Trans. I 11. $336-350(1975)$

Collins, R. J., Husain, D., and Donovan, R. J., "Kinetic and Spectroscopic Studies of $\sigma_{2}\left(a^{1} \Delta_{g}\right)$ by Time-Resolved Absorption Spectroscopy in the Vacuum Ultra-violet," J. Chem. Soc., Faraday Trans. II 69, 145-157 (1973)

Coltharp, R. N., Worley, S. D., and Potter, A. E., "Reaction Rate of Vibrationally Excited Hydroxyl with Ozone," Appl. Eptics 10, 1786-1789 (1971)

Colussi, A. J., Singleton, D. L., Irwin, R. S., and CretanoviE, R. J., "Absolute Rates of Axygen ( ${ }^{3} P$ ) Atom Reactions with Benzene and Toluene," J. Phys. Chem. 79, 1900-1903 (1975)

Cox, R. A., "The Photolysis of Nitrous Acid in the Presence of Carbon Monoxide and Sulphur Dioxide," J. Photochem. 3 , 291-304 (1974)

Cox, R. A., "The Photolysis of Gaseous Nitrous Acid - A Technique for ebtaining Kinetic Data on Atmospheric Photooxidation Reactions,"Int. J. Chem. Kinet., Symp. No. 1, 379-398 (1975)

Cox, R. A., and Derwent, R. G., "Kinetics of the Reaction of Ho with Nitric Oxide and Nitrogen Dioxide," J. Photochem. 4, 139-153 (1975)

Cox, R. A., and Derwent, R. G., "The Ultraviolet Absorption Spectrum of Gaseous Nitrous Acid," J. Photochem. 6, 23-34 (1976)

Cox, R. A., Derwent, R. G., and Holt, P. M. "The Photo-gxidation of Ammonia in the Presence of $\mathrm{NO}$ and $\mathrm{NO}_{2}$," Chemosphere, No. 4, 201-205 (1975)

Cox, R. A., Derwent, R. G., and Holt, P. M., "Relative Rate Constants for the Reactions of $\theta \mathrm{H}$ Radicals with $\mathrm{H}_{2}, \mathrm{CH}_{4}, \mathrm{C} \theta$, NG and HGN at Atmospheric Pressure and $296 \mathrm{~K}, " \mathrm{~J}$. Chem. Soc.. Faraday Trans. I 72. 2031-2043 (1976)

Cox, R. A., Derwent, R. G., Holt, P. M., and Kerr, J. A., "Photo- Bxidation of Methane in the Presence of NO and $\mathrm{N}_{2}$," J. Chem. Soc. Faraday Trans. I $72,2044-2060$ (1976)

Cupitt, L. T., and Glass, G. P., "Reactions of SH with Atomic oxygen and Hydrogen," Int. J. Chem. Kinet., Symp. No. 1, 39-50 (1975)

Daubendiek, R. L., and Calvert, J. G., "A study of the $\mathrm{N}_{2} \boldsymbol{\theta}_{5}-\mathrm{SO}_{2}-\boldsymbol{\theta}_{3}$ Reaction System," Environ. Lett. 8, $103-116$ (1975)

Davidson, J.A., Schiff, H. I., Streit, G. E., McAfee, J. R., Schmeltekopf, A. L., and Howard, C. J., "Temperature Dependence of o( ${ }^{1}$ D) Rate Constants for Reactions with $\mathrm{N}_{2} \theta, \mathrm{H}_{2}, \mathrm{CH}_{4}, \mathrm{HCl}$, and $\mathrm{NH}_{3}$," J. Chem. Phys. 67, 5021-5025 (1977)

Davidson, J. A., and Thrush, B. A., "Reaction of Oxygen Atoms with Methyl and Ethyl Nitrites," J. Chem. Soc., Faraday Trans. I I1. 2413-2420 (1975)

Davis, D. D.. "Absolute Rate Constants for Elementary Reactions of Atmospheric Importance: Results from the University of Maryland Gas Kinetics Laboratory," Report 3 (Univ. of Maryland, College Park, Ma. 20742, 1976)

Davis, D. D.. Braun, W., and Bass, A. M.. "Reactions of $\mathrm{Cl}^{2} \mathrm{P}_{3 / 2}$ : Absolute Rate Constants for Reaction ith $\mathrm{H}_{2}, \mathrm{CH}_{4}, \mathrm{C}_{2} \mathrm{H}_{6}, \mathrm{CH}_{2} \mathrm{Cl}_{2}, \mathrm{C}_{2} \mathrm{Cl}_{4}$, and co $\mathrm{C}_{6} \mathrm{H}_{12}$ " Int. J. Chem. Kinet. 2 , $101-114$ (1970)

Davis, D. D., Bollinger. W., and Fischer, S., "A Kinetics study or the Reaction of the $\sigma H$ Free Radical with Aromatic Compounds. I. Absolute 
Rate Constants for Reaction with Benzene and Toluene at $300^{\circ} \mathrm{K}, " \mathrm{~J}$. Phys. Chem. 79, 293-294 (1975)

Davis, D. D., Fischer, S., and Schiff, R., "Flash Photolysis-Resonance Fluorescence Kinetics Study: Temperature Dependence of the Reactions बH $\rightarrow \mathrm{CO} \rightarrow \mathrm{CO}_{2}+\mathrm{H}$ and $\mathrm{OH} \cdot \mathrm{CH}_{4} \rightarrow \mathrm{H}_{2} \theta+\mathrm{CH}_{3}, " \mathrm{~J}$. Chem. Phys. 61, 2213$2219(1974)$

Davis, D. D., Fischer, S., Schiff, R., Watson, R. T., and Bollinger, W., "A Kinetics Study of the Reaction of GH Radicals with Two $\mathrm{C}_{2}$ Hydrocarbons: $\mathrm{C}_{2} \mathrm{H}_{4}$ and $\mathrm{C}_{2} \mathrm{H}_{2}$, J. Chem. Phys, 63, 1707-1712 (1975)

Davis, D. D., Herron, J. T., and Hule, R. E., "Absolute Rate constants for the Reaction $\theta\left({ }^{3} \mathrm{P}\right) \cdot \mathrm{NH}_{2} \rightarrow \mathrm{NG} \rightarrow \mathrm{A}_{2}$ Over the Temperature Range 230-339K," J. Chem. Phys, 58, $530-535(1973)$

Davis, D. D., Machado, G., Conaway, B., Oh, Y., and Watson, R., "A Temperature Dependent Kinetics study of the Reaction of $\mathrm{GH}$ with $\mathrm{CH}_{3} \mathrm{Cl}, \mathrm{CH}_{2} \mathrm{Cl}_{2}$, $\mathrm{CHCl}_{3}$, and $\mathrm{CH}_{3} \mathrm{Br}, " \mathrm{~J}$. Chem. Phys, 65, 126801274 (1976)

Davis, D. D., Payne, M., and Stief, L., "The Hydroperoxyl Radical in Atmospheric Chemical Dynamics: Reaction with Carbon Monoxide, "Science 179. $280-282(1973)$

Davis, D. D., Prusazcyk, J., Dwyer, M., and Kim, P., "A Stopoflow Time of Flight Mass Spectrometry Kinetics study. Reaction of Ozone with Nitrogen Dioxide and Sulfur Dioxide," J. Phys. Chem. 78, 1775-1779 (1974)

Davis, D. D., Wone, W., and Lephardt, J., "A Laser Flash Photolysis-Resonance Fluorescence Kinetic Study: Reaction of $\theta\left({ }^{3} P\right)$ with $\theta_{3}, "$ Chem. Phys.

Lett. 22, 273-278 (1973)

Davis, D. D., Wong, W., and Schiff, R., "A Dye Laser Flash Photolysis Kinetics study of the Reaction of Ground-state Atomic Oxygen with Hydrogen Peroxide," J. Phys. Chem. 78, 463-464 (1974)

DemerJian, K. L., Kerr, J. A., and Calvert, J. G., "The Mechanism of Photochemical Smogi Formation," Adv. Environ. Sci. Technol. 4, 1-262 (1974)

J. N, Pitts, Jr., and R. L, Metcalf, editors, Wiley-Interscience

DeNore, W. B., "Rate Constants for the Reactions of Hydroxyl and Hydroperoxyl with Ozone," Science 180,735-737 (1973)

DeMore, W. B., "Arrhenius Constants for the Reactions of Ozone with Ethylene and Acetylene," Int. J. Chem. Kinet. 1, 209-220 (1969)

DeMore, W. B., "Rate Constant Ratio for the Reactions of $\theta H$ with 3 and Co," Int. J. Chem. Kinet., Symp. No. 1, 273-279 (1975)

DeMore, W. B., and Tschulkow-Roux, E., "Temperature Dependence of the Reactions of $\theta \mathrm{H}$ and $\mathrm{H \theta}_{2}$ with $\theta_{3}, " \mathrm{~J}$. Phys. Chem. 78, 1447-1451 (1974)

Dillemuth, F. J., Skidmore, D. R., and Schubert, C. C., "The Reaction of Ozone with Methane," J. Phys. Chem. 64, 1496-1499 (1960)

Donovan, R. J., Husain, D., and Kirsch, L, J., "Reactions of Atomic Oxygen. Part 1: The Rate of the Reaction $\theta^{+} \theta_{2}+M \rightarrow \theta_{3} \cdot M$ ( $M=$ He, Ar and Kr)," Trans. Faraday Soc. 66, 2551-2559 (1570)

Dubinsky, R. N., McKenney, D. J., "Determination of the Rate Constant of the $\theta-\mathrm{H}_{2} \rightarrow \theta H$ - $\mathrm{H}$ Reaction using Atomic Oxygen Resonance Fluorescence and the Air Afterglow Techniques," Can. J. Chem. 53, 3531-3541 (1975)

Fettis, G. C., and Knox, J. H., "Rate Constants of Halogen Atom Reactions," G. Porter, Ed., Progress in Reaction Kinet. (Pergamon Press) 2, 1-38 (1964)

Findlay, F. D., and Snelling, D. R., "Temperature Dependence of the Rate Constant for the Reaction $\theta_{2}\left({ }^{1} \Delta_{g}\right) \cdot \theta_{3} \rightarrow 2 \theta_{2} \cdot \theta, " J$. Chem. Phys. 54 . 2750-2755 (1971) 
Findlay, F. D., and Snelling, D. R., "Collisional Deactivation of " ${ }_{2}\left({ }^{1} \Delta_{g}\right)$ " J. Chem. Phys, 55, 545-551 (1971a)

Fisher, E. R., and McCarty, M., Jr., "Study of the Reaction of Electronically Excited Oxygen Molecules with Carbon Monoxide," J. Chem. Phys. $45.781-$ 784 (1966)

Fletcher, I. S., and Husain, D,. "The Collisional Quenching of Electronically Excited Oxygen Atoms, $\theta\left(2^{\mathrm{l}} \mathrm{D}_{2}\right)$ by the Gases $\mathrm{NH}_{3}, \mathrm{H}_{2} \mathrm{\theta}_{2}, \mathrm{C}_{2} \mathrm{H}_{6}, \mathrm{C}_{3} \mathrm{H}_{8}$, and $\mathrm{C}\left(\mathrm{CH}_{3}\right)_{4}$ Using Time-Resolved Attenuation of Atomic Resonance Radiation," Can. J. Chem. 54. 1765-1770 (1976a)

Fletcher, I. S., and Husain, D., "Absolute Reaction Rates of Oxygen( $2^{1} D_{2}$ ) with Halogenated Parafins by Atomic Absorption Spectroscopy in the Vacuum Ultraviolet," J. Phys. Chem. 80, 1837-1840 (1976b)

Foner, S. N., and Hudson, R. L.. "Mass Spectrometry of the Ho, Free Radical," J. Chem. Phys. 3ㅌ, 2681-2688 (1962)

Francis, P. D., "The Production of Oxygen Atoms in a Microwave Discharge and the Recombination Kinetics in a Gas Flow System," Brit. J. Appl. Phys. 2, 1717-1730 (1969)

Gardiner, W. C., Jr., Mallard, W. G., McFarland, M., Morinaga, L., Gwen, J. H., Rawlins, W. T., Takeyama, T., and Walker, B. F., "Elementary Reaction Rates from PostoInductionoPeriod Profiles in Shock-Initiated Combustion," Symp. Combust. 14th (Combustion Institute, Pittsburgh, 1973) 61-72

Gardiner, W. C., Jr., Mallard, W. G., and Owen, J. H., "Rate Constant of $\theta \mathrm{H}+\mathrm{H}_{2}=\mathrm{H}_{2} \theta+\mathrm{H}$ from 1350 to $1600 \mathrm{~K}, " \mathrm{~J}$. Chem. Phys. $\underline{\underline{0}}, 2290-2295$ $(1974)^{2}$

Genring, M., Hoyermann, K., Schacke, H., and Wolfrum, J., "Direct Studies of Some Elementary steps for the Formation and Destruction of Nitric oxide in the HoN-O System," Symp. Combust. 14th (Combustion Institute, Pittsburgh, 1973) 99-105

Gnormley, J. A., Ellsworth, R. L., and Hochanadel, C. J., "Reaction of Excited Oxygen Atoms with Nitrous Oxide. Rate Constants for Reacion of ozone with Nitric Oxide and with Nitrogen Dioxide," J. Phys. Chem. 77, 13411345 (1973). Erratum: 78, 2698 (1974)

Giachardi, D. J., Harris, G. W., and Wayne, R. P., "Excited State Formation in the $H$ - $\theta_{2}$ System," Chem. Phys. Lett. $32,586-588$ (1975)

Giachardi, D. J., Harris, G. W., and Wayne, R. P., "Energy Transfer from Excited $\mathrm{NG}_{2}$ * to Molecular Exygen," J. Chem. Soc.. Faraday Trans. II 72. $619.630(1976)$

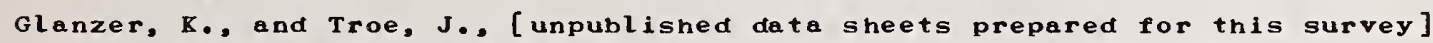
Ecole Polytechnique Federale de Lausanne (1973)

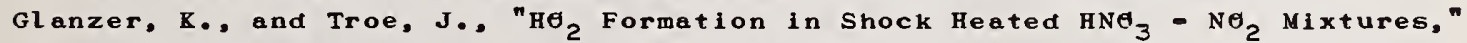
Ber. Bunsenges. Phys. Chem. 79, 465-469 (1975)

Glavas, S., and Toby, S., "The Reaction between Ozone and Hydrogen Sulfide: Kinetics and Effect or Added Gases," Am. Chem. Soc., ACS Symp. Series $17,122-131$ (1975)

Gordon, R. J., and Lin, M. C., "The Reaction of Nitric Oxide with vibrationally Excited Gzone," Chem. Phys. Lett. 22, 262-268 (1973)

Gordon, R. J., and Iin, M. C.. "The Reaction of Nitric Oxide with Vibrationally Excited Ozone. II.." J. Chem. Phys. 64, 1058-1064 (1976)

Gordon, S., and Mulac, W. A., "Reaction of the $\theta H\left(x^{2} \Pi\right)$ Radical Produced by the Pulse Radiolysis of Water Vapor," Int. J. Chem. Kinet., Symp. No. 1 , $289-299$ (1975) 
Gordon, S., Mulac, W., and Nangia, P., "Pulse Radiolysis or Ammonia Gas. II. Rate of Disappearance of the $\mathrm{NH}_{2}\left(\mathrm{x}^{2} \mathrm{~B}_{1}\right)$ Radical," J. Phys. Chem. 75,20870 $2093(1971)$

Gorse, R. A., and Volman, D. H., "Photochemistry of the Gaseous Hydrogen Peroxide-Carbon Monoxide System: Rate Constants for Hydroxyl Radical Reactions with Hydrogen Peroxide and Isobutane by Competitive Kinetics," J. Photochem. 1, 1-10 (1972)

Gorse, R. A., and Volman, D. H., "Photochemistry of the Gaseous Hydrogen Peroxide-Carbon Monoxide System II: Rate Constants for Hydroxyl Radical Reactions with Hydrocarbons and for Hydrogen Atom Reactions with Eydrogen Peroxide," J. Photochem. ․, 115-122 (1974)

Graham, R. A., and Johnston, H. S., "Kinetics of the Gas-Phase Reaction between Gzone and Nitrogen Dioxide," J. Chem. Phys. 60, 4628-4629 (1974)

Graham, R. A., and Johnston, H. S., "The Photochemistry of $\mathrm{Ng}_{3}$ and the Kinetics of the $\mathrm{N}_{2} \theta_{5}-\theta_{3}$ System," J. Phys. Chem. $82,254-268$ (1978)

Graham, R. E., and Gutman, D., "Neasurement of Branching Ratios for the $\theta+\mathrm{CS}_{2}$ - $\theta \mathrm{CS}$ - S Reaction," Photochem. Conference 12th (National Bureau of Stanards, Washington, D. C. 20234,1976 , H4-1

Gray, D., Lissi, E., and Heicklen, J., "The Reaction of Hydrogen Peroxide with Nitrogen Dioxide and Nitric Oxide," J. Phys. Chem, 76, 1919-1924 (1972)

Greiner, N. R., "Hydroxyl Radical Kinetics by kinetic spectroscopy. III. Reactions ith $\mathrm{H}_{2} \theta_{2}$ in the Range $300-458^{\circ} \mathrm{K}, "$ J. Phys. Chem. 72. $406-410(1968)$

Greiner, N. R., "Hydroxyl Radical Kinetics by Kinetic Spectroscopy. v. Reactions with $\mathrm{H}_{2}$ and $\mathrm{c} \theta$ in the Range $300-500^{\circ} \mathrm{K}, " \mathrm{~J}$. Chem. Phys. 51 , $5049-5051$ (1969)

Greiner, N. R., "Hydroxyl Radical Kinetics by Kinetic Spectroscopy. VI. Reactions with Alkanes in the Range $300-500^{\circ} \mathrm{K}, " \mathrm{~J}$. Chem. Phys. 53. $1070-1076(1970)$

Greiner, N. R., "Hydroxyl Radical Kinetics by Kinetic Spectroscopy. VII. The Reaction with Ethylene in the Range $300-500^{\circ} \mathrm{K}, " \mathrm{~J}$. Chem. Phys. 53, 12841285 (1970a)

Hack, W., Hoyermann, K., and Wagner, H. Gg., "Reaktionen des Hydroxylradikals mit Ammoniak und Hydrazin in der gasphase," Ber. Bunsenges. Physik. Chem. 78, 386-391 (1974)

Hack, W., Hoyermann, K., and Wagner, H. Gg., "The Reaction $\mathrm{NO}^{+} \mathrm{H}_{2} \rightarrow$

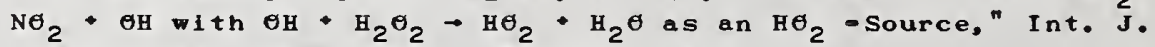
Chem. Kinet., Symp. No. 1, 329-339 (1975)

Hamilton, E. J., Jr., "Water Vapor Dependence of the Kinetics of the SelfReaction of $\mathrm{H}_{2}$ in the Gas Phase," J. Chem. Phys. 63, 3682-3683 (1975)

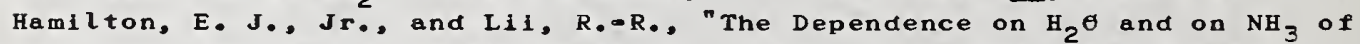
the Kinetics of the self-Reaction of $\mathrm{H}_{2}$ in the Gas-Phase Formation of $\mathrm{H}_{2}{ }^{\cdot} \mathrm{H}_{2} \theta$ and $\mathrm{H} \theta_{2} \mathrm{NH}_{3}$ Complexes, Int. J. Chem. Kinet. 2, 875-885 (1977)

Hampson, R. F., (editor), "Survey of Photochemical and Rate Data for Twentyo Elght Reactions of Interest in Atmospheric Chemistry," J. Phys. Chem. Ref. Data 2, No. 2 pgs. 267-312 (1973). These are revised versions of dat: sheets originally distributed as NBS Reports 10692 and 10828.

Hampson, R. F.. (editor), "Chemical Kinetics Data Survey VI. Photochemical and Rate Data for Twelve Gas Phase Reactions of Interest for Atmospheric Chemistry,"NBSIR 73-207 (1973a)

Hancock, G., Lange, W., Lenzi, M., and Welge, K. H., "Laser Fluorescence of $\mathrm{NH}_{2}$ and Rate Constant Measurement of $\mathrm{NH}_{2}+\mathrm{N} \theta, "$ Chem Phys. Lett. 33. $168-172(1975)$ 
Hansen, D. A., Atkinson, R., and Pitts, J. N., Jr., "Rate Constants for the Reaction of $\sigma H$ Radicals with a Series of Aromatic Hydrocarbons," J. Phys. Chem. 79, 1763-1766 (1975)

Hansen, I., Hoinghaus, K., Zetzsch, C., and stuhl, F., "Detection of NH( $X^{3} \Sigma^{-}$) by Resonance Fluorescence in the Pulsed Vacuum UV Photolysis of $\mathrm{NH}_{3}$ and Its Application to Reactions of NH Radicals," Chem. Phys. Lett. 42, 370-372 (1976)

Hanst, P. L., Stephens, E. R., Scott, W. E., and Doerr, R. C., "Atmospheric

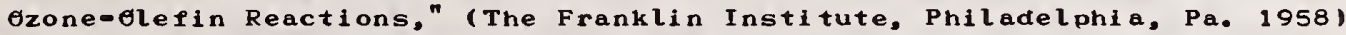

Harker, A., and Johnston, H. S,. "Photolysis of Nitrogen Dioxide to Produce Transient $\sigma, \mathrm{N}_{3}$ and $\mathrm{N}_{2} \sigma_{5}, " \mathrm{~J}$. Phys. Chem. 77, $1153-1156$ (1973)

Harris, G. W., and Wayne, R. P., "Reaction of Hydroxyl Radicals with $\mathrm{NO}, \mathrm{N \theta}_{2}$ and $\mathrm{S \theta}_{2}$, J. Chem. Soc.. Faraday Trans. I I1, 610-617 (1975)

Hastie, D. R., Freeman, C, G., McEwan, M. J., and Schiff, H, I., "The Reactions of Gzone with Methyl and Ethyl Nitrites," Int. J. Chem. Kinet. 8. $307-313(1976)$

Heicklen, J., "Gas-Phase Reactions of Alkylperoxy and Alkoxy Radicals," Adv. Chem. Ser. (Amer. Chem. Soc,. Washington) 76, 23-39 (1968)

Heicklen, J., "Photochemical and Rate Data for Methyl Nitrite, Methoxy and Methylperoxy," Chemical Kinetics Data Survey v. Sixtyosix Contributed Rate and Photochemical Data Evaluations on Ninetyofour Reactions, NBSIR 73-206, D. Garvin, Ed., National Bureau of Standards, Washington, D.C. (1973) pages $43-48$

Heidner, R. F., III, and Husain, D., "Electronically Excited Oxygen Atoms, $\theta\left(2^{1} D_{2}\right)$. A Time-Resolved Study of the Collisional Quenching by the Gases $\mathrm{H}_{2}, \mathrm{D}_{2}, \mathrm{NO}, \mathrm{N}_{2} \mathrm{\theta}, \mathrm{NO}_{2}, \mathrm{CH}_{4}$, and $\mathrm{C}_{3} \mathrm{O}_{2}$ Using Atomic Absorption Spectroscopy in the vacuum Ultraviolet, "Int J. Chem. Kinet. $5,819-831$ (1973)

Heidner, R. F., III, Husain, D., and wiesenfeld, J. R., "Kinetic Investigation of Electronically Excited Axygen Atoms, $\sigma\left(2^{1} \mathrm{D}_{2}\right)$, by Time-resolved Attenuation of Atomic Resonance Radiation in the Vacuum Ultraoviolet. Part 2.-Collisional Quenching by the Atomospheric Gases $\mathrm{N}_{2}, \theta_{2}, \mathrm{C}$, $\mathrm{C \theta}_{2}, \mathrm{H}_{2} \sigma$ and $\theta_{3}, " \mathrm{~J}$. Chem. Soc., Faraday Trans. II 69, $927-938$ (1973)

Herron, $J . T .$, and Huie, R. E., "Rate Constants for the Reactions of Ozone with Ethene and Propene, from 235.0 to $362.0 \mathrm{~K}, " \mathrm{~J}$. Phys. Chem. 78, 20850 2088 (1974)

Herron, J. T., and Huie, R. E., "Rate Constants for the Reactions of Atomic Gxygen ( $\sigma^{3} P$ ) with organic Compounds in the Gas Phase," J. Phys. Chem. Ref. Data 2, 467-518(1973)

Herron, J. T., and Huie, R. E., "Rates of Reaction of Atomic Oxygen. II. Some $C_{2}$ to $C_{8}$ Alkanes," J. Phys. Chem. 73, 3327-3337 (1969)

Hikida, T., Eyre, J. A., and Dorfman, L. M., "Pulse Radiolysis Studies. XX. Kinetics of Some Addition Reactions of Gaseous Hydrogen Atoms by Fast Lyman-a Absorption Spectrophotometry," J. Chem. Phys. 54, 3422-3428 (1971)

Hippler, $H_{,}$, and Troe, J., "Hochdruckbereich der Rekombination $\boldsymbol{\theta}_{2} \rightarrow$ $\theta_{3}$, Ber. Bunsenges. Physik. Chem. $75,27-32$ ( 1971 )

Hippler, H., Schippert, C, and Troe, J., "Photolysis of No 2 and Collisional Energy Transfer in the Reactions $\theta+\mathrm{N} \theta \rightarrow \mathrm{NO}_{2}$ and $\mathrm{O}+\mathrm{N \theta _{2 }} \rightarrow \mathrm{N \theta _{3 }}$." Int. J. Chem. Kinet., Symp. No. 1, 27-38 (1975)

Hochanadel, C. J., Ghormley, J. A., and Ggren, P. J., "Absorption Spectrum and Reaction Kinetics of $\mathrm{He}_{2}$ Radical in the Gas Phase," J. Chem. Phys. 56. $4426-4432$ (1972) 
Hochanadel, C. J.,. Ghormley, J. A., Boyle, J. W., and Ogren, P. J,, "Absorption Spectrum and Rates of Formation and Decay of the $\mathrm{CH}_{2} \mathrm{\theta}_{2}$ Radical," J. Phys. Chem. 81, 3-7 (1977)

Hogan, L. G., and Burch, D. S., "A Measurement of the Rate Constant for the Reaction $\theta \cdot \theta_{2}+\theta_{2} \rightarrow \theta_{3}+\theta_{2}, " J$. Chem. Phys. 65, 894-900 (1976)

Hollinden, G. A., Kurylo, M. J., and Timons, R. B., "Electron Spin Resonance study of the kinetics of the Reaction of ( $^{3} P$ ) Atoms with H, "J."Phys. Chem. 74, 588-591 (1970)

Holmes, H. H., and Daniels, F., "The Photolysis of Nitrogen Oxides: $\mathrm{N}_{2} \theta_{5}$ $\mathrm{N}_{2} \mathrm{O}_{4}$ and $\mathrm{NO}_{2}$ " "J.Amer. Chem. Soc. $56,630-637$ (1934)

Holt, R. B., Mclane, C. K., and Oldenberg, O., "Ultraviolet Absorption Spectrum of Hydrogen Peroxide," J. Chem. Phys, 16, 225-229 (1948). Erratum: 16, 638 ( $1 \subseteq 48)$

Horowitz, A., and Calvert, J. G., "The Wavelength Dependence of the Quantum Ffficiencies of the Primary Processes in

Formaldehyde Photolysis at $25^{\circ} \mathrm{C}$, " typescript, to be published Int. J. Chem. Kinet. (1978a)

Horowitz, A., and Calvert, J. G., "The Quantum Efficiency of the Primary Processes in Formaldenyde Photolysis at 3130 and $25^{\circ} \mathrm{C}, "$ typescript, to be published Int. J. Chem. Kinet. (1978b)

Houston, P. L., and Moore, C. B., "Formaldehyde Photochenistry: Appearance Rate, Vibrational Relaxation, and Energy Distribution of the co Product," J. Chem. Phys, 65, 757-770 (1976)

Howard, C. J., "Rate Constants for the Gasophase Reactions of OH Radicals with Ethylene and Halogenated Ethylene Compounds," J. Chem. Phys. $6 \underline{5}$, $4771-4777$ (1976)

Howard, C. J.. "Kinetics of the Reaction of $\mathrm{H \theta}_{2}$ with $\mathrm{N \theta}_{2}$ " J. Chem. Phys. 67, 5258-5263 (1977)

Howard, C. J., and Evenson, K. M., "Laser Magnetic Resonance Study of the Gas Phase Reactions of $\theta \mathrm{H}$ with $\mathrm{CO}, \mathrm{Ne}$, and $\mathrm{NA}_{2}$, $\mathrm{J}$. Chem. Phys. 61. $1943-1952$ (1574)

Howard, C. J., and Evenson, K. M., "Rate Constants for the Reactions of OH with Ethane and some Halogen Substituted Ethanes at $296 \mathrm{~K}, "$ J. Cher. Phys, 64., 4303-4306 (1976)

Howard, C. J., and Everson, K. M., "Rate Constants for the Reactions OH with $\mathrm{CH}_{4}$ and Fluorine, Chlorine, and Bromine Substituted Methanes at $296 \mathrm{~K}$," J. Chem. Phys. 64, 197-202 (1976)

Howard, C. J., and Evenson, K. M., "Kinetics of the Reaction of $\mathrm{HO}_{2}$ - $1 \mathrm{th}$ NG," Geophys. Res. Lett. 4. 437-440 (1977)

Huestis, D. L., Black, G., Edelstein, S. A., and Sharpless, R. L., "Fluorescence and Quenching of $\theta_{2}\left({ }^{1} \Delta_{g}\right)$ and $\left[\theta_{2}\left({ }^{1} \Delta_{g}\right)\right]_{2}$ in Liquid $\theta x y g e n, " J . C h e m$. Phys. $\underline{60}, 4471-4474(1974)$

Hui, K.-K., and Cool, T. A., "Experiments Concerning the Laser Enhanced Reaction Between Vibrationally Excited $\theta_{3}$ and Na," J. Chem. Phys. 68, 1022 (1978)

Huie, R. E., and Herron, J. T., "Temperature Dependence of the Rate Constants for Reactions of Ozone with Some Glefins," Int. J. Chem. Kinet., Symp. No. $1,165-181$ (1975)

Huie, R. E.. and Herron, J. T., "The Rate Constant for the Reaction $\theta_{3}+N_{2} \rightarrow$ $\theta_{2}$ - $\mathrm{NO}_{3}$ Over the Temperature Range $259-362^{\circ} \mathrm{K}, "$ Chem. Phys. Lett. 27 , $411-414(1974 \mathrm{a})$ 
Huie, R. E., Herron, J. T, and Davis, D. D., "Absolute Rate Constants for the Reaction $\theta+\theta_{2}+\mathrm{M} \rightarrow \theta_{3}+M$ Gver the Temperature Range $200-346^{\circ} \mathrm{K}, "$ J. Phys. Chem. 76, 2653-2658 (1972)

Husain, D., Kirsch, L. J., and Donovan, R. J., "A Kinetic Study of $\theta\left(2^{3} P_{J}\right.$, by Atomic Absorption Spectroscopy Following the Flash Photolysis of Ozone," J. Photochem. 1, 69-73 (1972)

Jacob, A., and Winkler, C. A., "Kinetics of the keactions of Oxygen Atoms and Nitrogen Atoms with Sulphur Trioxide," J, Chem. Soc., Faraday

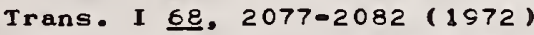

James, F. C., Kerr, J. A., and Simons, J. P., "A Direct Measurement of the Rate of Reaction of the Methyl Radical with Sulphur Dioxide,"J. Chem. Soc. Faraday Trans. I $69,2124-2129$ (1973)

Japar, S. M.. Hu, C. H., and Niki, H., "Rate Constants for the Reaction of Gzone with Glefins in the Gas Phase," J. Phys, Chem. Iㅇ, 2318 (1974)

Jayanty, R. K. M., Simonaitis, R., and Heicklen, J., "The Reaction of $\mathrm{NH}_{2}$ with $\mathrm{N \theta}_{2}$," (Department of Chemistry and Ionosphere Research Laboratory, Penn. State Univ., Pa. 16802, 1975d)

Jayanty, R. K. M., Simonaitis, R.. and Heicklen, J., "The Reaction of $\mathrm{NH}_{2}$ with No and $\theta_{2}, " J$. Phys. Chem. $80,4 \equiv 3-437$ (1976b)

Johnston, H. S., "Gas Phase Reaction Rate Theory," The Ronald Press Co., New York (1966), Chapter 1

Johnston, H. S.. "Gas Phase keaction Kinetics of Neutral Axygen Species," NBS-NSRDS-20, 1968, (supt. of Documents, U.S. Govt. Printing office, Washington, D.C. 20402)

Johnston, H. S., "Catalytic Reduction of Stratospheric Ozone by Nitrogen Gxides," Adv. Environ. Sci. Technol. 4, 263-380 (1974), J. N. Pitts, Jr., and $R$. L. Metcalf, editors, Wiley-Interscience

Johnston, H. S., private communication (1573)

Johnston, H. S., Chang, S.-G., and Whitten, G.. "Photolysis of Nitric Acid Vapor," J. Phys. Chem. $78,1=7$ (1974)

Johnston, H. S., and Graham, R., "Gas Phase Ultraviolet Absorption Spectrum of Nitric Acid Vapor," J. Phys. Chem. 77, 62-63 (1973)

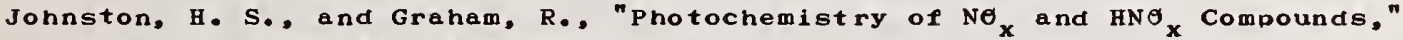
Can. J. Chem. 52, 1415-1423 (1974)

Jonnston, H. S., Morris, E. D., Jr., and Van den Bogaerde, J., "Molecular Modulation Kinetic spectrometry. Cles and Cl $\theta_{2}$ Radicals in the Photolysis of Chlorine in Oxyken," J. Amer. Chem. Soc. 91, 7712-7727 (1969)

Johnston, H. S., and Selwyn, G. S., "New Cross Sections for the Absorption of Near Ultraviolet Radiation by Nitrous Oxide ( $\mathrm{N}_{2} \theta$ ), Geophys. Res. Lett. 2, 549-551 (1975)

Jones, I. T. N., and Bayes, K. D., "Photolysis of Nitrogen Dioxide," J. Chem. Phys. 59, 483604844 (1973)

Jones, E. J., and Wulf, $\theta$. R.. "Absorption Coefficient of Nitrogen Pentoxide in the Ultraviolet and the Visible Absorption Spectrum of $\mathrm{Ng}_{3}, " \mathrm{~J}$. Chem. Phys. 5, 873-877 (1937)

Kajimoto, G., and Cretanovie, R. J., "Formation of Nitrous oxide in the Reaction of $\theta\left({ }^{1} \mathrm{D}_{2}\right)$ Atoms with Nitrogen," J. Chem. Phys. 64, 1005-1015 (1976)

Kerr. J. A., and Parsonage, M. J., "Evaluated Kinetic Data on Gas Phase Addition Reactions: Reactions of Atoms and Radicals with Alkenes, Alkynes and Aromatic Compounds," (Butterworths, 1572) 
Klemm, R. B., and Stief, L. J., "Absolute Rate Parameters for the Reaction of Ground State Atomic Oxygen with Carbonyl Sulfide," J. Chem. Phys. 61, $4900-4906$ ( 1974$)$

Kl emm, R. B., Payne, W. A., and Stief, L. J.. "Absolute Rate Parameters for the Reaction of Atomic Hydrogen with $\mathrm{H}_{2} \mathrm{\theta}_{2}, "$ Int. J. Chem. Kinet.. Symp. No. 1 , $61-72(1975)$

Kondratiev, V. N., "Konstanty Skorosti Gazofaznykh Reaktsij Spravochnik," (Izdatelstro "Nauka", Moskva, 1970); also issued as "Rate Constants of Gas Phase Reactions, Reference Book," R. M. Fristrom, Ed., Translated by L. J. Holtschlag CeM-72-10014, Office of Standard Reference Data, NBS ( 1972 ) (Distributed by National Technical Information Service, Spring* field, Va, 22151)

Kurylo, M. J., Hollinden, G. A., LeFevre, H, F., and Timmons, R, B., "ESR Study of the Kinetics of the Reactions of D Atoms and O Atoms with $\mathrm{NH}_{3}$," J. Chem. Phys. 51, 4457-4501 (1969)

Kurylo, M. J.. "Absolute Rate Constants for the Reaction $H+\theta_{2}+M-$ $\mathrm{H}_{2}$ * M Ever the Temperature Range $203-404 \mathrm{~K}, " \mathrm{~J}$. Phys. Chem. 76, 3518$3526(1972)$

Kurylo, M. J., "Kinetics of the Reactions $\left.\mathrm{GH}_{(\mathrm{v}}=0\right)+\mathrm{NH}_{3} \rightarrow \mathrm{H}_{2} \theta+\mathrm{NH}_{2}$ and $\theta \mathrm{H}(\mathrm{v}=0)+\theta_{3} \rightarrow \mathrm{HO}_{2}+\theta_{2}$ at $298^{\circ} \mathrm{K}, "$ Chem. Phys. Letts. 23. $467-$ 471 (1973)

Kurylo, M. J., "Flash Photolysis Resonance Fluorescence Investigation of the Reaction of $\theta\left({ }^{3} P\right)$ Atoms with $\mathrm{ClONO}_{2}$ " "Chem. Phys. Lett. 49 , $467=470(1977)$

Kurylo, M. J., and Braun, W., "Flash Photolysis Resonance Fluorescence Study of the Reaction $\mathrm{Cl}+\theta_{3} \rightarrow \mathrm{Cl} \theta * \theta_{2}$ over the Temperature Range 213-298 K," Chem. Phys, Lett. 37, 232-235 (1976)

Kurylo, M. J., and Manning, R. G., "Flash Photolysis Resonance Fluorescence Investigation of the Reaction of $\left.\mathrm{Cl}^{2} \mathrm{P}\right)$ Atoms with Clon ${ }_{2}$, "Chem. Phys. Lett. $48,279=283$ (1977)

Kurylo, M. J., Braun, W., and Xuan, C. N., "Infrared Laser Enhanced Reactions: Temperature Resolution of the Chemical Dynamics of the $\theta_{3}$ NO Reaction System," J. Chem. Phys, 62, 2065-2071 (1975)

Kurylo, M. J., Peterson, N. C., and Braun, W., "Absolute Rate of the Reaction $\mathrm{H}+\mathrm{H}_{2} \mathrm{~S}, " \mathrm{~J}$. Chem. Phys. $54,943-946$ (1971)

Laufer, A. H., and Bass, A. M., "Rate Constants of the Combination of Methyl Radicals with Nitric Oxide and Oxygen," Int. J. Chem. Kinet. I, 639-648 (1975)

Lee, J. H.. Michael, J. V., Payne, W. A., and Stief, L. J., "Absolute Rate of the Reaction of Hydrogen Atoms with fzone from $219-360 \mathrm{~K}$," paper presented at 13 th Informal Conference on Photochemistry, Clearwater Beach, Florida, Jan. 1978

Lee, J. H., Michael, J. V., Payne, W. A., Stief, L. J., and Whytock, D. A., "Absolute Rate of the Reaction of $C l\left({ }^{2} P\right)$ with Molecular Hydrogen from 200-500 K," J. Chem. Soc., Faraday Trans. I 73, 1530-1536 (1977)

Lesclaux, R., van khe, P., Dezauzier, P., and Soulignac, J. C., "Flash Photolysis studies of the Reaction of $\mathrm{NH}_{2}$ Radicals with No," Chem. Phys. Lett. 35, 493-497 (1975)

Leu, M-T., and DeMore, W. B., "Rate Constants at $295 \mathrm{~K}$ for the Reactions of Atomic Chlorine with $\mathrm{H}_{2} \theta_{2}, \mathrm{H}_{2}, \theta_{3}, \mathrm{CH}_{4}$ and $\mathrm{HNO}_{3}$, Chem Phys, Lett. 41 , $121-124(1976)$

Leu, M.-T., and DeMore, W. B., "Rate Constant for the Reaction of Atomic Bromine with Ozone," Chem. Phys. Lett. 48, 317-320 (1977) 
Leu, M. T., Lin, C. L., and DeMore, W. B., "Rate Constant for Formation of Chlorine Nitrate by the Reaction Clo * $\mathrm{NO}_{2}$ * $\mathrm{M}_{3}$ " J. Phys.

Chem. 81, 190-195 (1977)

Lewis, R. S., Tang, K. Y,, and Lee, E. K. C., "Photoexcited Chemilumio nescence Spectroscopy: Detection of Hydrogen Atoms Produced from Single vibronic Level Photolysis of Formaldehyde $\left(\pi^{1} A_{2}\right)$, J. Chem. Phys. 65, 2910-2911 (1976)

Lewis, R. S., and Lee, E. K. C., "Photolysis of Formaldehyde-Butene Mixtures at Low Pressures: Quantum Yield of Radical Decomposition Process in Formaldenyde," typescript, Dept. of Chemistry, Univ. of California, Irvine, CA (1577)

Lin, C. L., and DeMore, W. B., " $\theta\left({ }^{1} D\right)$ Production in Ozone Photolysis Near 31001," J. Photochem. 2, 161-164 (1973)

Lloyd, A. C.. "Evaluated and Estimated Kinetic Data for Gas Phase Reactions of the Hydroperoxyl Radical," Int. J. Chem. Kinet. 6,1690228 (1974) Supersedes NBS Report 10447

Lundell, O. R., Ketcheson, R. D., and Schiff, H. I., "The Production of ( $^{3} P$ ) Atoms, Free From Excited Molecules, and Their Reaction with $\theta_{3}, " S y m p$. Combust. 12th (Combustion Institute, Pittsburgh, 1969) 307-311

Mack, G. P. R., and Thrush, B. A., "Reaction of Axygen Atoms with Carbonyl Compounds. Part 1. Formaldehyde," J. Chem. Soc., Faraday Trans. I 69, $208-215(1973)$

Mandelman, M., and Carrington, T., "The fovalue of the No $\delta(0,0)$ Band by the Line Absorption Method," J. Quant. Spectrosc. Radiat. Transfer 14, 509-521 (1974)

Manning, R. G., Braun, w., and Kurylo, M. J., "The Effect of Infrared Laser Excitation on Reaction Dynamics: $\theta+\mathrm{C}_{2} \mathrm{H}_{4}^{+}$and $\theta+\theta C S^{+}, " J$. Chem. Phys. 65, 2609-2615 (1976)

Manning, R. G., and Kurylo, M. J.. "Flash Photolysis Resonance Fluorescence Investigation of the Temperature Dependencies of the Reaction of $\mathrm{Cl}\left({ }^{2} \mathrm{P}\right)$ Atoms with $\mathrm{CH}_{4}, \mathrm{CH}_{3} \mathrm{Cl}, \mathrm{CH}_{3} \mathrm{~F}, \mathrm{CH}_{3} \mathrm{~F}^{*}$, and $\mathrm{C}_{2} \mathrm{H}_{6}, "$ J. Phys, Chem. 81, 291-296 (1977)

Margitan, J. J., Kaufman, F., and Anderson, J. G., "The Reaction of $\mathrm{HH}_{\mathrm{H}} \mathrm{wh}_{\mathrm{CH}}$," Geophys. Res. Lett. 1, 80-81 (1974)

Margitan, J. J., Kaufman, F., and Anderson, J. G., "Kinetics of the Reaction OH $+\mathrm{HNO}_{3}-\mathrm{H}_{2} \mathrm{\theta}+\mathrm{NG}_{3}$," Int. J. Chem. Kinet., Symp. No. 1, $281-287$ (1575)

Marling, J., "Isotope Separation of Gxygen-17, Oxygen-13, Carbon-13, and Deuterium by Ion Laser Induced Formaldehyde Photopredissociation," J. Chem. Phys. 66,4200-4225 (1977)

McCrumb, J. L., and Kaufman, F., "Kinetics of the $\theta+\theta_{3}$ Reaction," J."Chem. Phys. 57, $1270-1276$ ( 1972 )

McKenzie, A., Mulcahy, M. F. R., and Steven, J. R., "Kinetics of Decay of Hydroxyl Radicals at Low Pressure," J. Chem. Phys. 59, 3244-3254 (1973)

McQuigg, R. D., and Calvert, J. G., "The Photodecomposition of $\mathrm{CH}_{2} \theta_{,} \mathrm{CD}_{2} \theta$ CHD $\theta$ and $\mathrm{CH}_{2} \theta-\mathrm{CD}_{2} \theta$ Mixtures at Xenon Flash Lamp Intensities," J. Amer. Chem. Soc. 91, 1550-1599 (1569)

Meaburn, G. M., Perner, D., LeCalve, J., and Bourene, M., "A Pulsed-Radiolysis Study of Atomic Oxygen Reactions in the Gas Phase,"J. Phys. Chem. 72 , 3520-3925 (1968)

Meagher, J. F., and Heicklen, J., "The Photolysis of Hydrogen Peroxide in the Presence of Carbon Monoxide," J. Photochem. 3 , 455-466 (1975) 
Michael, J. V., Payne, W. A,, and Fhytock, D. A., "Absolute Rate Constants for $\theta \mathrm{NG}+\mathrm{M}(=\mathrm{He}, \mathrm{Ne}, \mathrm{Ar}, \mathrm{Kr}) \rightarrow \mathrm{N} \theta_{2} \cdot \mathrm{M}$ from 217 to $500 \mathrm{~K}, "$

J. Chem. Phys. Es, 4830-4834 (1976)

Milks, D., and Natula, R. A., "A Single-Pulse shock-Tube Study of the Reaction between Nitrous Gxide and Carbon Monoxide," Symp. Combust. lath (Combustion Institute, Pittsburgh, 1973) 83.96

Miller, R. G., and Lee, E. K. C., "Single Vibronic Level Photochenistry of Formaldehydes in the $X^{1} \mathrm{~A}_{2}$ State: Radiative and Non-Radiative Processes in $\mathrm{H}_{2} \mathrm{C} \theta$, $\mathrm{HDCC}$ and $\mathrm{D}_{2} \mathrm{C} \sigma, "$ typescript, Dept. of Chemistry, Univ, of California, Irvine, CA (1977)

Nolina, L. T., Spencer, J. E., and Molina, H. J., "The Rate constant for the

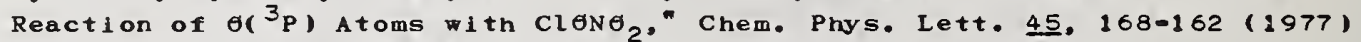

Molina, M. J. (1977), "Cross Sections and Quantun Yields," in Chapter 1 of NASA Reference Publication 1010 "Chlorofluoromethanes and the Stratosphere", R. D. Hudson, Ed., August 1977

Moortgat, G. K., and Allen, E. R., "The Room Temperature Reactions of HydrogenAtoms in Molecular Oxygen," National Center for Atmospheric Research, Boulder (1973). Abstract of unpublished work.

Moortgat, G. K., Slemr, F., and Warneck, P., "Kinetics and Mechanism of the Reaction $\mathrm{H}+\mathrm{CH}_{3}$ GNO," Int. J. Chem. Kinet., , 249-265 (1977)

Moortgat, G. K., Slemr, F., Seiler, W., and Warneck, P.. "Wavelength Dependence of Relative and Absolute Quantum Yields in the Near Uv Photolysis of Formaldehyde at $25^{\circ} \mathrm{C}$." paper presented at 13 th Informal Conference on Photochemistry, Clearwater Beach, Florida, Jan. (1978)

Morley, C., and Smith, I. W. N., "Rate Measurements of Reactions of $\mathrm{HH}_{\mathrm{H}} \mathrm{by}$ Resonance Absorption. Part I. Reactions of $\theta \mathrm{H}$ wh $\mathrm{NO}_{2}$ and No,"

J. Chem. Soc., Faraday Trans. II 68, 1016-1030 (1972)

Morris, E. D., Jr., and Niki, H., "Mass Spectrometric study of the Reaction of Hydroxyl Radical with Formaldetide," J. Chem. Phys. 55, 1991-1992 (1971)

Morris, E. D., Jr., and Niki, H., "Reaction of Methyl Radicals with Atomic exygen," Int. J. Chem. Kinet. $5,47-53$ (1972)

Morris, E. D., Jr., and Niki, H, "Reaction of Dinitrogen Pentoxide with Water," J. Phys. Chem. 77, 1929-1932 (1973)

Morris, E. D., Jr., Stedman, D. H., and Nik1, H., "Mass Spectrometric study of the Reactions of the Hydroxyl Radical with Ethylene, Propylene, and Acetaldehyde in a Discharge-Flow System," J. Amer. Chem. Soc. c3. $3570-3572(1971)$

Koy, J., Bar-Ziv, E., and Gordon, R. J., "Temperature Dependence of the Laser Enhanced Reaction $\mathrm{N} \theta \cdot \theta_{3}(001) \rightarrow \mathrm{N}_{2}\left({ }^{2} \mathrm{~B}_{1,2}\right) \cdot \theta_{2}, "$ J. Chem. Phys. 66, 5439-5446 (1977)

Mulcahy, M. F. R., and Williams, D. J., "Kinetics of combination of oxygen Atoms with Oxyeen Molecules," Trans. Faraday Soc. 64, 59-70 (1968)

Mulvihill, J. N., and Phillips, L. F., "Products of Reaction of NH with NA," Chem. Phys. Lett. 35 , 327-329 (1975)

NASA (1977). "Chlorofluoromethanes and the Stratosphere," R. D. Hudson, Ed., NASA Reference Publication 1010, August 1977, Chapter 1. Recommendations of the Laboratory Measurements Committee for the NASA chlorofluoromethane assessment.

Nicholas, J. E., and Norrish, R. G. W., "Some Reactions in the Chlorine and Oxygen System Studied by Flash Photolysis," Proc. Roy. Soc. (Iondon) A 307, $391-397$ ( 1968) 
Ggryzlo, E. A., and Thrush, B. A., "Vibrational Excitation of No in its Collisional Quenching of $\theta_{2}\left({ }^{1} \Delta_{g}\right), "$ Chem. Phys. Lett. $23,34-36$ (1973)

Overend, R., and Paraskevopoulos, G." "The Question of a Pressure Effect in the Reaction $\sigma H$ - CA at Room Temperature," Chem. Phys. Lett. 49. 109-111 (1977)

Gverend, R., and Paraskevopoulos, G., "Rates of $\theta H$ Radical Reactions. III. The Reaction $\mathrm{OH}_{\mathrm{H}} \cdot \mathrm{C}_{\chi \mathrm{H}_{4}}$. M at $256^{\circ} \mathrm{K}, " \mathrm{~J}$. Chem. Phys. 67, 674-679 (1977)

Gverend, R., Paraskevopoulos, G., and Black, C., "Rates of of Radicals Reactions. II. The Combination Reaction OH * NO * $M_{0}$ "J. Chem. Phys. 64, 4149-4154 (1976)

fverend, R., Paraskevopoulos, g., and Cretanovie, R. J., "Rates of $\theta H$ Radical Reactions. I. Reactions with $\mathrm{H}_{2}, \mathrm{CH}_{4}, \mathrm{C}_{2} \mathrm{H}_{6}$, and $\mathrm{C}_{3} \mathrm{H}_{8}$ at 295 K," Can. J. Chem. 53. 3374-3382 (1975)

Parkes, D. A., "The Roles of Alkylperoxy and Alkoxy Radicals in Alkyl Radical Oxidation at Room Temperature," Symp. Combust. 15th (Combustion Institute, Pittsburgh, 1975) 755-805

Parkes, D. A., "Oxidation of Methyl Radicals at Room Temperature,"Int. J. Chem. Kinet. . $451-469$ (1977)

Pastrana, A., and Carr, R. W.., Jr., "Rate of the Reaction of Hydroxyl Radical with Acetylene," Int. J. Chem. Kinet. 6, 587-595 (1974)

Pate, C. T., Finlayson, B. J., and Pitts, J. N., Jr., "A Long Path Infrared Spectroscopic study of the Reaction of Methylperoxy Free Radicals with Nitric Oxide," J. Am. Chem. Soc. 96. 6554-6558 (1974)

Payne, H. A.. Stief, L. J., and Davis, D. D., "A Kinetics Study of the Reaction of $\mathrm{HO}_{2}$ with $\mathrm{SO}_{2}$ and $\mathrm{NO}, " \mathrm{~J}$. Amer. Chem. Soc. 95, 7614-7619 (1973)

Peeters, J., and Mahnen, G., "Reaction Mechanisms and Rate Constants of Elementary steps in Methane-fxygen Flames," Symp. Combust. 14th (Combustion Institute, Pittsburgh, 1973) 133-141

Penzhorn, R-D., Gusten, H., Schurath, U., and Becker, K. H., "Quenching of Singlet Molecular Oxygen by Some Atmospheric Pollutants," Environ. Sci. Technol. 8,907 (1974)

Penzhorn, R-D., Gusten, B., Schurath, U., and Becker, K. H., "Halogenierte Kohlenwasserstoffe in der Atmosphare: Geschwindigkeitskonstanten fur die Loschung von Metastabilen Sauerstoffmolekulen," Staub Reinhalt Luft 35 , 95-98 (1975)

Perry, R. A., Atkinson, R., and Pitts, J. N., Jr., "Rate Constants for the Reaction of $\Theta \mathrm{H}$ Radicals wth $\mathrm{CHFCl}_{2}$ and $\mathrm{CH}_{3} \mathrm{Cl}$ over the Temperature Range $298-423^{\circ} \mathrm{K}$, and with $\mathrm{CH}_{2} \mathrm{Cl}_{2}$ at $298^{\circ} \mathrm{K}, " \mathrm{~J}$. Chem. Phys. 64 . $1618-1620(1976)$

Perry, R. A.. Atkinson, R., and Pitts, J. N., Jr., "Rate Constants for the Reactions $\sigma \mathrm{H} \rightarrow \mathrm{H}_{2} \mathrm{~S} \rightarrow \mathrm{H}_{2} \theta+\mathrm{SH}$ and $\theta \mathrm{H} \cdot \mathrm{NH}_{3} \rightarrow \mathrm{H}_{2} \theta+\mathrm{NH}_{2}$ Over the Temperature Range 297-427" K," J. Chem. Phys. 64, 3237-3239 (1976a)

Perry, R. A., Atkinson, R., and Pitts, J. N., Jr., "Rate Constants for the Reaction of $\theta H$ Radicals with n-Butane over the Temperature Range 297-420 K," J. Chem. Phys. 64. 5314-5316 (1976b)

Perry, R. A., Atkinson, R., and Pitts, J. N., Jr., "Kinetics and Mechanism of the Gas Phase Reaction of $\theta H$ Radicals with Aromatic Hydrocarbons Over the Temperature Range 296-473 K," J. Phys. Chem. 81, 296-304 (1977)

Perry, R. A., Atkinson, R., and Pitts, J. N., Jr., "Kinetics of the Reactions of $\theta \mathrm{H}$ Radicals with $\mathrm{C}_{2} \mathrm{H}_{2}$ and $\mathrm{C} \theta$,"J. Chem. Phys. 67. $5577-5584$ (1977a) 
Phillips, L. F., and Schiff, H. I., "Mass Spectrometric Studies of Atomic Reactions. III. Reactions of Hydrogen Atoms with Nitrogen Dioxlde and with Gzone," J. Chem. Phys. 37., 1233-1238 (1962)

Pruss, F. J., Jr., slagle, I. R., and Gutman, D., "Determination of Branching Ratios for the Reaction of axygen Atoms with Ethylene," J. Phys. Chem. 78 . $663-665(2974)$

Ravishankara, A. R, Davis, D. D., Smith, G., Tesi, G., and Spencer, J., "A Study of the Chemical Degradation of CleNe, in the Stratosphere," Geophys. Res. Lett. 4. 7-9 (1977)

Rawlins, W. T., and Gardiner, W. C., Jr., "Rate Constant of $\theta H+\theta H=H_{2} \theta+\theta$ from 1500 to $2000 \mathrm{~K}, " \mathrm{~J}$. Chem. Phys, 60, 4676-4681 (1974)

Ridley, B. A., Davenport, J. A., Stief, L. J., and Welge, K. H., "Absolute Rate Constant for the Reaction $\mathrm{H} \cdot \mathrm{H}_{2} \mathrm{C \theta}, " \mathrm{~J}$. Chem. Phys. 57, 520-523 (1972)

Rowland, F. S., Spencer, J. E., and Molina, M. J., "Stratospheric Formation and Photolysis of Chlorine Nitrate," J. Phys. Chem. 80, 2711-2713 (1976)

Sauer, M. C., Jr., "A Pulse-Radiolysis Study of the Dependence of the Reaction of Atomic Gxygen with Axygen on the Nature of the Third Body," J. Phys. Chem. 71, 3311-3313 (1967)

Schmatjko, K. J., and Wolfrum, J., "Reaction of Flash Photolytically Produced CN(x ${ }^{2}$, , $)$ Radicals with $6\left({ }^{3} P\right)$ Atoms," Photochem. Conference 12 th (National Bureau of Standards, Washington, D. C. 20234 1976) R5-1

Schmidt, C., and Schiff, H. I., "Reactions of $\theta_{2}\left({ }^{1} \Delta_{g}\right.$ ) with Atomic Nitrogen and Hydrogen," Chem. Phys. Lett. 233, 3390342 (1973)

Schofield, K.. "The Rate of Destruction of $\sigma_{2}\left({ }^{1} \Delta_{g}\right.$ ) by Atomic Hydrogen," Intern. J. Chem. Kinet. 4. 255-263 (1972)

Schofleld, K., "Evaluated Chemical Kinetic Rate Constants for Various Gas Phase Reactions," J. Phys. Chem. Ref. Data 2, 25-84 (1973)

Schott, G. L., Getzinger, R. W., and Seitz, W. A., "Transient Oxygen Atom Yields in $\mathrm{H}_{2} \theta_{2}$ Ignition and the Rate Coefficient for $\mathrm{O}$ - $\mathrm{H}_{2}$ - $\mathrm{OH}$ - $\mathrm{H}$," Int. J. Chem. Kinet. $6,921-943$ (1574)

Selwyn, G., Podolske, J., and Johnston, H. S., "Nitrous Oxide Ultraviolet Absorption Spectrum at Stratospheric Temperatures," Geophys. Res. Lett. 4, 427-430 (1977)

Sie, B. K. T., Simonaitis, R, and Heicklen, J., "The Reaction of GH with ce," Int. J. Chem. Kinet. $8,85-98$ (1976a)

Sie, B. K. T., Simonaitis, R., and Heicklen, J," "The Reaction of gH with No," Int. J. Chem. Kinet. 8 , 99-106 (1976b)

Simonaitis, R., and Heicklen, J.. "The Reactions of $H_{2}$ with Carbon Monoxide and Nitric Gxide and the Reaction of $\sigma\left({ }^{1} D\right)$ with Water," J. Phys. Chem. 77 , $1096-1102(1973 a)$

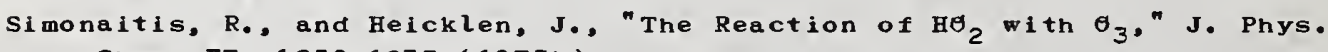
Chem. 77, 1932-1935 (1973b)

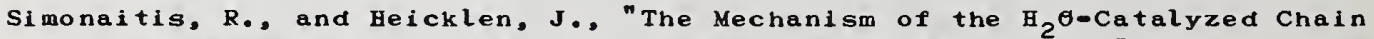
Photodecomposition of $\theta_{3}$ and the Reaction of $H\left(\right.$ with $\theta_{3}$ " J. Photochem. 2 , $309-314$ ( $1973 \mathrm{C})$

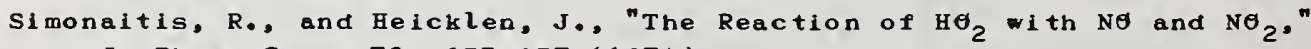
J. Phys. Chem. 78, 653-657(1974)

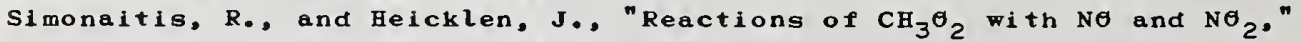
J. Phys. Chem. 78, 2417-2421 (1974)

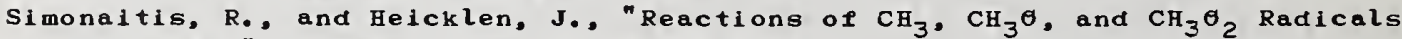
with $\theta_{3}, " J$. Phys. Chem. 79, 298-302 (1975)

Simonaitis, R., and Heicklen, J., "Reactions of $\mathrm{H}_{2}$ with $\mathrm{Ne}$ and $\mathrm{NO}_{2}$ and of OH with NO,"J. Phys. Chem. 80, 1-7 (1976a) 
Simonaltis, R., and Heicklen, J., "The Temperature Dependence or the Reactions of $\mathrm{HO}_{2}$ with $\mathrm{NO}$ and $\mathrm{NO}_{2}$," Int. J. Chem. Kinet. 10, 67-87 (1978)

Singleton, D. L., and Cvetanovir, R. J., "Temperature Dependence of the Reactions of Oxygen Atoms with Olefins," J. Am. Chem. Soc. 98, 6812-6819 (1976)

Singleton, D. L., Furuyama, S., Cretanovit, R. J., and Irwin, R. S., "Temperature Dependence of the Rate Constants for the Reactions $\theta\left({ }^{3} P\right.$ ) - 2,3-Dimethyl-2-Butene and $\theta\left({ }^{3} P\right)+N \theta+M$ Determined by a Phase Shift Technique," J. Chem. Phys. 63, 1003-1007 (1975)

slack, M. W.. "Rate Coefficient for $\mathrm{H}+\theta_{2}+\mathrm{M}=\mathrm{H} \theta_{2}+\mathrm{M}$ Evaluated from Shock Tube Measurements of Induction Times," Combust. Flame 28. 241-249 (1977)

Slagle, I. R., Pruss, F. J., Jr., and Gutman, D., "Kinetics into the Steady State. I. Study of the Reaction of Oxygen Atoms with Methyl Radicals," Int. J. Chem, Kinet. $6,111-123$ (1974)

slagle, I. R., Gilbert, J. R., and Gutman, D., "Kinetics of the Reaction between Oxyeen Atoms and Carbon Disulfide," J. Chem. Phys. 62 , $740-709$ (1974)

Slagle, I. R., Graham, R. E., Gilbert, J. R., and Gutman, D., "Direct Determination of the Rate Constant for the Reaction of Oxygen Atoms with Carbon Monosulphide," Chem. Phys. Lett. 32, 184-186 (1975)

Slanger, T. G., and Black, G., "Reaction Rate Measurements of $\theta\left({ }^{3} P\right.$ ) Atoms by Resonance Fluorescence. I. $\theta\left({ }^{3} P\right)+\theta_{2}+M \rightarrow \theta_{3}+M$ and $\theta\left({ }^{3} P\right)+N \theta+M \rightarrow$ $\mathrm{NG}_{2}$ + M," J. Chem. Phys. 53, 3717-3721 (1970)

slaneer, T. G., and Black, G., "G( $\left.{ }^{1} S\right)$ Quenching by $\theta\left({ }^{3} P\right), " J$. Chem. Phys. 64, 3763.3766 (1976a)

Slanger, $T$. G., and Black, G., "Temperature Dependence for Quenching of $\theta\left({ }^{i} s\right)$ by $\mathrm{N}_{2} \theta, " \mathrm{~J}$. Chem. Phys. $\underline{\underline{5}}, 2025-2026$ (1976b)

slanger, T. G., Wood, B. J., and Black, G.. "Investigation of the Rate Coefficient for $\theta\left({ }^{3} P\right)+N \theta_{2} \rightarrow \theta_{2}+N \theta, " I n t . J$. Chem. Kinet. 5, 615-620 (1973)

slanger. T. G., and welge, K. H., "Rate Constants for Reactions of $\theta\left({ }^{1} \mathrm{~s}\right), "$ in Chemical Kinetics Data Survey V. Sixty-six Contributed Rate and Photochemical Data Evaluations on Ninetyofour Reactions, NBSIR 73-206, D. Garvin, Ed., National Bureau of Standards, Washington, D.C., (1973) pages $22-32$

slemr, F., and Warneck, P., "Kinetics of the Reaction of Atomic Hydrogen with Methylhydroperoxide," Int. J. Chem. Kinet. $9,267-282(1977$ )

Smith, I. W. M., and Zellner, R., "Rate Measurements of Reactions of OH by Resonance Absorption Part 2. Reactions of $\theta \mathrm{H}$ wh $\mathrm{C} \theta_{\text {, }} \mathrm{C}_{2} \mathrm{H}_{4}$ and $\mathrm{C}_{2} \mathrm{H}_{2}$," J. Chem. Soc., Faraday Trans. II 69 , 1617-1627 (1973)

Smith, I. W. M., and Zellner, R., "Rate Measurements of Reactions of $\theta H$ by Resonance Absorption. Part 3. Reactions of eH with $\mathrm{H}_{2}$. $\mathrm{D}_{2}$. Hydrogen and Deuterium Halldes," J. Chem. Soc., Faraday Trans. II 70, 104501056 (1974a)

Smith, I. W. M., and Zellner, R., "Rate Measurements of Reactions of oH by Resonance Absorption. IV. - Reactions of OH with $\mathrm{NH}_{3}$ and $\mathrm{HNO}_{3}, "$ Int. J. Chem. Kinet., Symp. No. 1, 341-351 (1975)

Spencer, J. E., and Glass, G. P., "A Reexamination of the Reaction H $+\mathrm{HCl}=\mathrm{H}_{2}+\mathrm{Cl}, " \mathrm{~J}$. Phys. Chem. 79, 2329-2335 (1975)

Sperling, H. P., and Toby, S., "The Photochemical Decomposition of Gaseous Formaldehyde," Can. J. Chem. 51, 471-475 (1973)

Stedman, D. H., and Niki, H., "Kinetics and Mechanism for the Photolysis of Nitrogen Dioxide in Air," J. Phys. Chem. 77, 2604-2609 (1973) 
Stedman, D. H., Wu, C. H., and Niki, H., "Kinetics op Gas-Phase Reactions of Ozone with Some olefins," J. Phys. Chem. 77, 2511-2514 (1973)

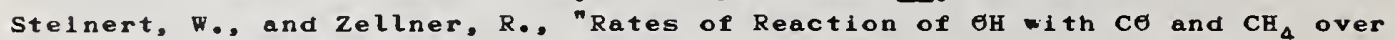
an Extended Temperature Range," Deuxieme Symp. European gur la Combustion (Orleans, 1975) 31-37

Stief, L. J., and Payne, W. A., "Absolute Rate Parameters for the Reaction of Atomic Hydrogen with Hydrazine," J. Chem. Phys. 64, 4892-4896 (1976)

Streit, G. E., and Johnston, H. S., "Reactions and Quenching of vibrationally Excited Hydroxyl Radicals," J. Chem. Phys. 64 95-103 (1976)

Streit, G. E., Howard, C. J., Schmeltekopf, A. L., Davidson, J. A., and Schiff, H. I. "Temperature Dependence of ( $^{1} D$ ) Rate Constants for Reactions with $\theta_{2}, \mathrm{~N}_{2}, \mathrm{C \theta}_{2}, \theta_{3}$, and $\mathrm{H}_{2}, " \mathrm{~J}$, Chem. Phys. $\underline{6}$, $4761-4764(1976)^{2}$

Stunl, F., "Absolute Rate Constant for the Reaction $\theta \mathrm{H}+\mathrm{NH}_{3} \rightarrow \mathrm{NH}_{2} \bullet \mathrm{H}_{2} \theta$," J. Chem. Phys. 59, 535-537 (1973a)

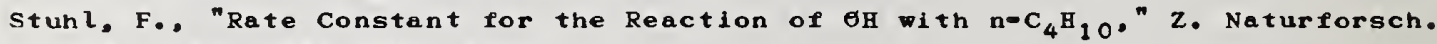
Pt A 28, 1383-1384 (1973b)

Stuhl, F., "Determination of Rate Constants for the Reactions of or with Propylene and Ethylene by a Pulsed Photolysis-Resonance Fluorescence Method," Ber. Bunsenges. Physik. Chem. $77,674-677$ (1973c).

Stunl, F.. "Determination of the Rate Constant for the Reaction GH * ${ }_{2}$ by a Pulsed Photolysis - Resonance Fluorescence Method," Ber. Bunsenges. Physik. Chem. 78, 230-232 (1974)

Stuhl, F., and Niki, H., "Detection of Oxygen ( $3_{p}$ ) Atoms in Pulsed Vacuum UV Photolysis of NO and Its Application to O Atom Reactions," Chem. Phys. Lett. I, 197-200 (1970)

Stunl, F., and Niki, H,., Measurements of Rate Constants for Termolecular Reactions of $\left.\sigma^{3} \mathrm{P}\right)$ ith $\mathrm{N} \theta, \theta_{2}, \mathrm{C}, \mathrm{N}_{2}$, and $\mathrm{C}_{2}$ Using a Pulsed Vacuum-uv Photolysis-Chemiluminescent Method," J. Chem. Phys. 55, 3943-3953 (1971)

Stunl, F., and Niki, H., "Pulsed Vacuum-ur Photochemical study of Reactions of $\theta \mathrm{H}$ with $\mathrm{H}_{2}, \mathrm{D}_{2}$, and C Using a Resonance-Fluorescent Detection Method," J. Chem. Phys. 57. 3671-3677 (1972)

Takacs, G. A., and Glass, G. P.. "Reactions of Hydrogen Atoms and Hydroxyl Radicals with Hydrogen Bromide," J. Phys. Chem.77, 1060-1064 (1973)

Takacs, G. A., and Glass, G. P.. "Reactions of Hydroxyl Radicals 1 ith Some Hydrogen Halides," J. Phys. Chem. 77, 1948-1951 (1973)

Takacs, G. A., and Glass, G. P., "Reaction of Atomic Oxygen with Hydrogen Bromide," J. Phys. Chem. 77, 1182-1186 (1973)

Taylor, R. L., "High Temperature Reactions of Air" pages 5-154 to 5-157 in "The Natural Stratosphere of 1974 " Climatic Impact Assessment Program Monograph 1, DeT-TST-75-51, Department of Transportation, Washington D.C., September 1975

Thrush, B. A., Letter to D. Garvin (Sept. 1973) reevaluating work in his laboratory

Toby, F. S., and Toby, S., "Reaction between Gzone and Allene in the Gas Phase," Int. J. Chem. Kinet. 6, 417-428 (1974)

Toby, F. S., and Toby, S., "The Reaction of Gzone with 1,3-Butadiene and with Allene," Int. J. Chem. Kinet., Symp. No 1, 197-204 (1975)

Toby, F. S., Toby, S., and $\theta^{\prime}$ Neal, H. E.. "The Kinetics of the Gas-Phase Reaction between Ozone and Alkenes," Int. J. Chem. Kinet. 8, 25-35 (1976)

Trainor, D. W., and von Rosenberg, C. W., Jr., "Flash Photolysis study of the Gas Phase Recombination of Hydroxyl Radicals," J. Chem. Phys.61. 1010-1015 (1974) 
Trainor, D. W., and von Rosenberg, C. W.., Jr.. "Energy Partitioning in the

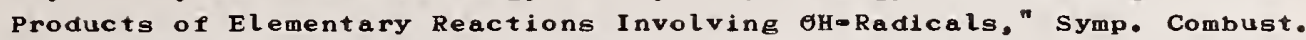
15th (Combustion Institute, Pittsburgh, 1975) 755-764

Tsang, w.. "Comparison between Experimental and Calculated Rate constants for Dissociation and Combination Reactions Involving Small Polyatomic Molecules," Int. J. Chem. Kinet. 5 , $947-563$ (1973)

Vardanyan, I. A., Dangyan, T. M., and Sachyan, G. A., "Rate Constants for the Reaction $\mathrm{HO}_{2}+\mathrm{CO} \rightarrow \mathrm{CO}_{2}+\sigma_{1}, "$ Dokl. Phys. Chem. 20.5, 632-634 (1972); tr. of Dokl. Akad. Nauk SSSR 205, 619-621 ( 1972 )

van den Bergh, H. E., and Callear, A. B., "Spectroscoplc Measurement of the Rate of the Gas-Phase Combination of Methyl Radicals with Nitric Gxide and Oxygen at $295 \mathrm{~K}, "$ Trans. Faraday Soc, 67, $2017-2024$ (1971)

Vandooren, J., Peeters, J., and Van Tikgelen, P. J.. "Rate Constant of the Elementary Reaction of Carbon Monoxide with Hydroxyl Radical," Symp. Combust. 15th (Combustion Institute, Pittsburgh, 1975) $745-752$

Volman, D. H., and Gorse, R. A.. "Rate Constant for the Reaction of Ho with Carbon Monoxide," J. Phys. Chem. Iㅇ. 3301-3302 (1972)

von Rosenberg, C. W.., Jr., and Trainor, D. W.. "vibrational Excitation of Gzone Formed by Recombination," J. Chem. Phys. 61, 2442-2456 (1974)

von Rosenbers, C. W., Jr., and Trainor, D. W.. "Excitation of Gzone formed by Recomblnation. II," J. Chem. Prys. 63, 5348-5353 (1975)

Wagner, H. Gg., Welzbacher, U., and Zellner, R., "Rate Measurements for the Reaction $\mathrm{H} \cdot \mathrm{Cl}_{2} \rightarrow \mathrm{HCl}+\mathrm{Cl}$ by Lyman- $\alpha$ Fluorescence," Ber. Bunsenges. Phys. Chem. 80, $902-908$ (1576)

Wagner, H. Gg., Welzbacher, U., and Zellner, R.. "Rate Measurements for the Reactions $\mathrm{H}+\mathrm{NO}_{2} \rightarrow \mathrm{OH}+\mathrm{NO}$ and $\mathrm{H}+\mathrm{N} \theta \mathrm{Cl} \rightarrow \mathrm{HCl}+\mathrm{NO}$

by Lyman-a Fluorescence," Ber. Bunsenges. Phys. Chem. 80. $1023-1027(1976)$

Walker, R. W., Ph.D., thesis, Univ, of London, Queen Mary College, 1973

Walker, R. W., Comments on "Gas-Phase Oxidation of Butene-2: The Role of Acetaldenyde in the Reaction," by Ray, D. J. Y., Diaz, R. R., and wassington, D. J., Symp. Combust. 14th (Combustion Institute, Pittsburgh, 1973) 265-266

Walker, R. W., "Rate Constants for Reactions in Gas-Phase Hydrocarbon Oxidation," Chapter 7 in "Gas Kinetics and Energy Transfer.

Volume $2^{n}$ A Specialist Periodical Report, The Chemical Society. London (1977)

Washida, N., and Bayes, K. D., "The Reactions of Methyl Radicals with Atomic and Molecular Oxygen," Int. J. Chem. Kinet. $8,777-794$ (1976)

Washida, N., Martinez, R. I., and Bayes, K. D.. "The Gxidation of Formyl Radicals," Z. Naturforsch. A $29,251-255$ (1 $\subseteq 74$ )

Watson, R. T., "Rate Constants for Reactions of Clo of Atmospheric Interest," J. Phys. Chem. Ref. Data $6,871-918$ (1977)

Watson, R., Machado, G., Fischer, S., and Davis, D. D., "A Temperature Dependence Kinetics study of the Reaction of $\mathrm{Cl}^{2} \mathrm{P}_{3 / 2}$ ) with $\theta_{3} \mathrm{CH}_{4}$ and $\mathrm{H}_{2} \boldsymbol{\theta}_{2}$, J. Chem. Phys. $65.5 .2126-2138$ (1976)

Watgon, R. T., Machado, G., Conaway, B., Wagner, S., and Davis, D. D., "A Temperature Dependent Kinetics Study of the Reaction of OH with $\mathrm{CH}_{2} \mathrm{ClF}, \mathrm{CHCl}_{2} \mathrm{~F}, \mathrm{CHClF}_{2}, \mathrm{CH}_{3} \mathrm{CCl}_{3} \mathrm{CH}_{3} \mathrm{CF}_{2} \mathrm{Cl}$, and $\mathrm{CF}_{2} \mathrm{ClCFCl}_{2}$ " J. Phys. Chem. 81, 256-262 (1977)

Weaver, J., Meagher, J., Shortridge, R., and Heicklen, J., "The Oxidation of Acetyl Radicals," J. Photochem. 4. 341-360 (1975) 
Wei, C. N., and Timmons, R. B., "ESR Study ot the Kinetics of the Reactions of $\theta\left({ }^{3} \mathrm{P}\right.$ ) Atoms with $\mathrm{CS}_{2}$ and GCS," J. Chem. Phys. 62, 3240-3245 (1975)

Festenberg, A. A., and deHaas, N., "Steadyostate Intermediate Concentrations and Rate Constants. Some He Results," J. Phys. Chem. 76, 1586-1593 (1972a)

Westenberg, A. A., and de Haas, N., "Measurement of the Rate Constant for $\mathrm{H}+\mathrm{H}_{2} \mathrm{CA} \rightarrow \mathrm{H}_{2}+\mathrm{HCO}$ at $297-652^{\circ} \mathrm{K}, " \mathrm{~J}$. Phys. Chem. ZE, 2213-2214 (1972b)

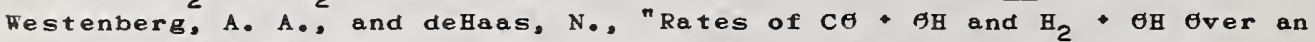
Extended Temperature Range," J. Chem. Phys. 58, 4061-4065 (1973a)

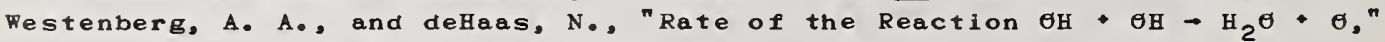
J. Chem. Phys. 58, 4066-4071 (1973b)

Westenberg, A. A., and deHaas, N., "Rates of H $\mathrm{CH}_{3} \mathrm{X}$ Reactions," J. Chem. Phys. 62, $3321-3325$ (1975a)

Hestenberg, A. A., and deHaas, N., "Reaction Rates of $\theta$ - $\mathrm{CH}_{3} \mathrm{Br}$ and $\theta$ - $\mathrm{CH}_{3} \mathrm{Cl}, "$ J. Chem. Phys, 62, 4477-4479 (1975b)

Westenberg, A. A., and deHaas, N., "Rate of the $\theta$ - so 3 Reaction," J. Chem. Phys. 6z, $725-730$ (1975c)

Westenberg, A. A., and deHaas, N., "Rate of the Reaction of $\theta+5 \sigma_{2}+M^{*}$ $\rightarrow \mathrm{S \theta}_{3} \cdot \mathrm{M}_{0}{ }^{n} \mathrm{~J}$. Chem. Phys. 63 , 5411-5415 (1975d)

Westenberg, A. A., Roscoe, J. M., and DeHaas, N., "Rate Measurements on $\mathrm{N}+\theta_{2}\left({ }^{1} \Delta_{\mathrm{g}}\right) \rightarrow \mathrm{N} \theta+\theta$ and $\mathrm{H}+\theta_{2}\left({ }^{1} \Delta_{\mathrm{g}}\right) \rightarrow \theta_{\mathrm{H}}+\theta, "$ Chem. Phys. Lett. $Z$, 597-599 (I970)

Whytock, D. A., Michael, J. V., and Payne, w. A., "Absolute Rate Constants for for $\theta+\mathrm{NO}+\mathrm{N}_{2} \rightarrow \mathrm{NO}_{2}+\mathrm{N}_{2}$ from $217-500 \mathrm{~K}, "$ Chem. Phys. Lett. 42 . $4 \epsilon 6=471(8976)$

Whytock, D. A., Lee, J. H., Michael, J. V., Payne, W. A., and Stief, L. J., "Absolute Rate of the Reaction of cl( ${ }^{2}$ ) with Methane from $200-500 \mathrm{X}$," J. Chem. Phys. 66, 2690-2695 (1977)

Whytock, D. A., Timmons, R. B., Lee, J. H., Michael, J. V., Payne, W. A., and Stief, L. J., "Absolute Rate of the Reaction of $\theta\left({ }^{3} P\right.$ ) with Hydrogen Sulfide Over the Temperature Range 263 to $495 \mathrm{~K}, " \mathrm{~J}$. Chem. Phys. $\underline{65}$. 2052-2055 (1976)

Wilson, Wm. E., Jr., "A Critical Review of the Gasophase Reaction Kinetics of the Hydroxyl Radical," J. Phys. Chem. Ref. Data 1. 535-573 (1972)

Wong, E. L., and Belles, F. E., "Rate Measurements for the Reaction of Hydrogen Chloride and Deuterium Chloride with Atouic Gxygen, "Lewis Research Center, NASA, Cleveland, Ohio, NASA Tech. Note, NASA TN-D-6495 (1971); Chem. Abstr. 76:18 $\$ 26 q$ (1972)

Wong, W., and Davis, D. D., "A Flash Photolysis-Resonance Fluorescence Study of the Reaction of Atomic Hydrogen with Molecular Axygen: $\quad H_{2}+\theta_{2} \rightarrow$

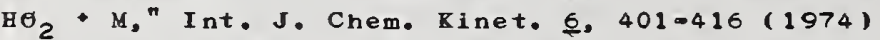

Wu, C. H., Morris, E. D., Jr., and Niki, H., "The Reaction of Nitrogen Dioxide with Ozone," J. Phys. Chem. 77, 2507-2511 (1973)

Hyrsch, D., Wendt, H, R., and Hunziker, H. E., "Modulation Xinetic Spectroscopy of $\mathrm{Hg} / \mathrm{H}_{2} / \theta_{2} / \mathrm{C \theta} \mathrm{M} 1 \mathrm{xtures:}$ Reactions of $\mathrm{Hg} H$ and $\mathrm{H} \sigma_{2}, " \mathrm{XI}$. Int. Symp. on Free Radicals (Berchtesgaden-Xonigssee, Sept. 4-7, 1973) Paper No. 36; see also, Ber. Bunsenges. Physik. Chem, 78, 204 (1974)

Yaron, M., von Engel, A., and Vidaud, F. H., "The Collisional Quenching of $\theta_{2}\left({ }^{1} \Delta_{g}\right)$ by NO and $\mathrm{CO}_{2}$, "Chem Phys. Lett. 37, 159-161 (1976)

Zahniser, M. S., Berquist, B. M., and Kaufman, F., "Kinetics of the Reaction $\mathrm{Cl}+\mathrm{CH}_{4} \rightarrow \mathrm{CH}_{3}$. HCl from 200 to $500 \mathrm{~K}$, "Int.J. Chem. Kinet. $10,15-29(1978)$ 
Zahniser. M. S., Chang, J. S., and Kaufman, F., "Chlorine Nitrate:

Kinetics of Formation by $\mathrm{Cl \theta} * \mathrm{NO}_{2}$. M and of Reaction with $\theta \mathrm{H}^{\text {, }}$

J. Chem. Phys. 67, 997-1003 (1977)

Zahniser, M. S., and Kaufman, F, "Kinetics of the Reactions of Clo with

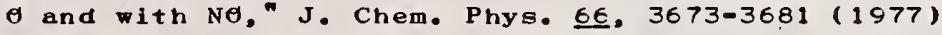

Zahniser, M. S., Kaufman, F., and Anderson, J. G., "Kinetics of the Reaction of OH with HCl," Chem. Phys. Lett, 27, 507-510 (1974)

Zahniser, M. S., Kaufman, F., and Anderson, J. G., "Kinetics of the Reaction $\mathrm{Cl}+\theta_{3} \rightarrow \mathrm{ClO}+\boldsymbol{\theta}_{2}, "$ Chem. Phys. Lett $37.226-231$ (1976)

Zellner, R., Erler, K., and Field, D., "Kinetics of the Recombination Reaction $\theta H \bullet H+M \rightarrow H_{2} \bullet * M$ at Low Temperatures," Symp. Combust. 16th (Combustion Institute, Pittsbureh, 1977) c39-948

Zellner, R., and steinert, w, "Flash Photolysis study of the Rate of the Reaction $\mathrm{OH} * \mathrm{CH}_{4} \rightarrow \mathrm{CH}_{3}+\mathrm{H}_{2} \mathrm{O}$ over an Extended Temperature Rane," Int. J. Chem. Kinet. 으. 397-409 (1976) 
Chemical Thermodynamic Properties of Selected Molecular Species

E. S. Domalski, D. Garvin, and D. D. Wagman

Two tables are provided showing chemical thermodynamic properties of selected molecular species of interest in stratospheric chemistry. In these tables there are sufficient data for the usual calculations of heats of reaction and equilibria.

The first table lists enthalpy and Gibbs energy of formation, entropy and heat capacity data. The information was extracted from "Selected Values of Chemical Thermodynamic Properties" (D. D. Wagman, et al, NBS Technical Note 270-3, January 1968) except as indicated in the Notes. The thermodynamic properties tabluated are:

1. enthalpy of formation at $\mathrm{OK}$ and $298.15 \mathrm{~K}$,

2. Gibbs energy of formation at $298.15 \mathrm{~K}$,

3. enthalpy increment between OK and 298.15K,

4. entropy at $298.15 \mathrm{~K}$,

5. heat capacity at constant pressure at $298.15 \mathrm{~K}$.

The values given for the heats of formation are based on experimental thermodynamic and spectroscopic measurements except where otherwise indicated; the values of $\mathrm{S}^{\circ}, \mathrm{C}_{\mathrm{p}}^{\circ}$ and $\mathrm{H}_{298}^{\circ} \mathrm{H}_{0}^{\circ}$ for gaseous species were calculated by standard statistical-mechanical equations, using corrections for vibrational anharmonicities, rotational stretching, and rotation-vibration interactions where these data were available.

The second table presents bond dissociation energies or bond strengths. These usually are the enthalpies of simple bond scission reactions. However, there are some cases in which there is a substantial contribution from rearrangement of electronic structure in the products (reducing the bond energy).

The values given here for some molecules may differ slightly from values adopted by the CODATA Task Group on Key Values for Thermodynamics (see CODATA Bulletin 17, published by the International Council of Scientific Unions Committee on Data for Science and Technology, January 1976). These differences will have no significant effect on the enthalpies or free energies of reaction calculated from the tables. 


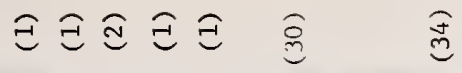

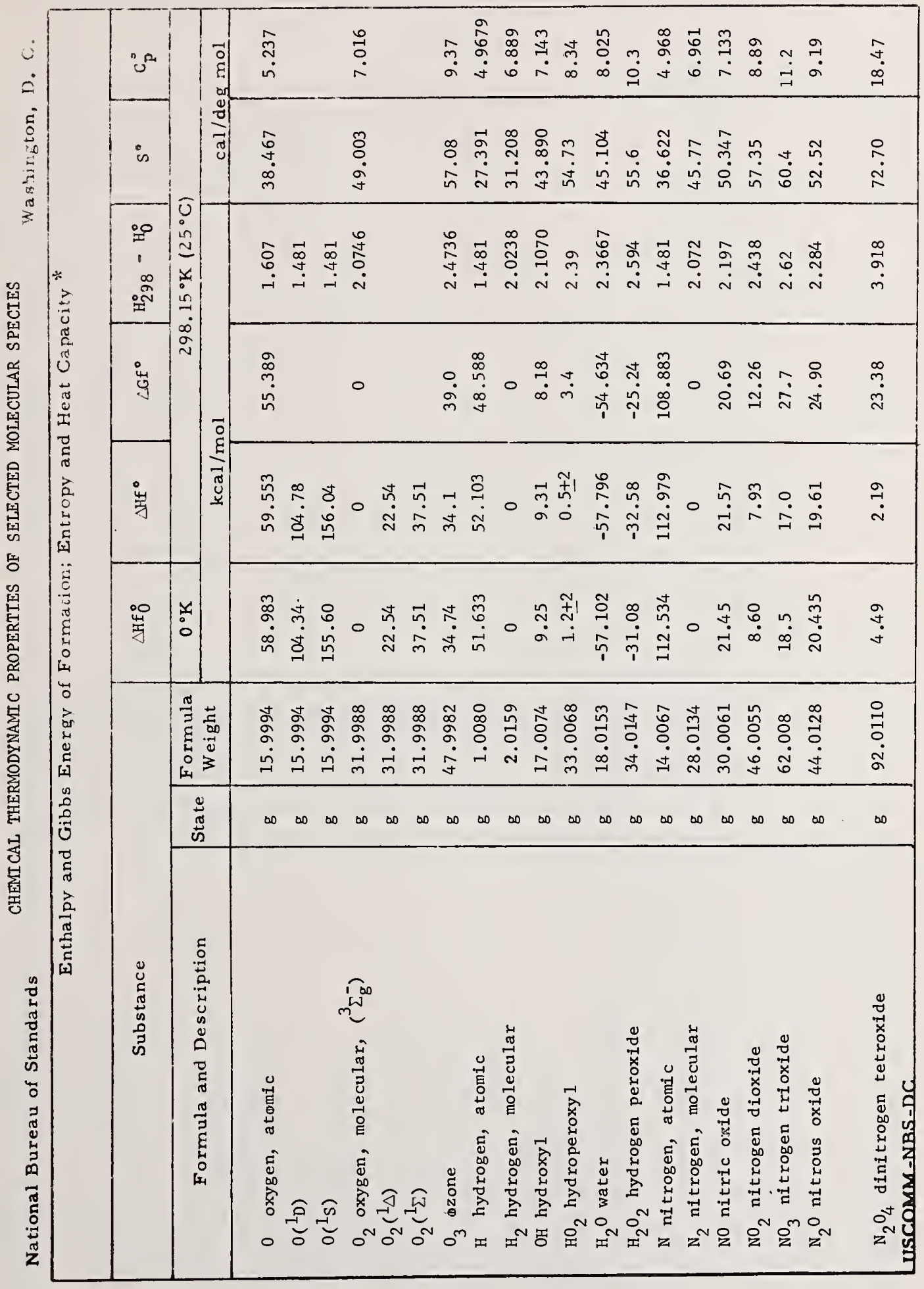




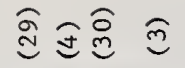

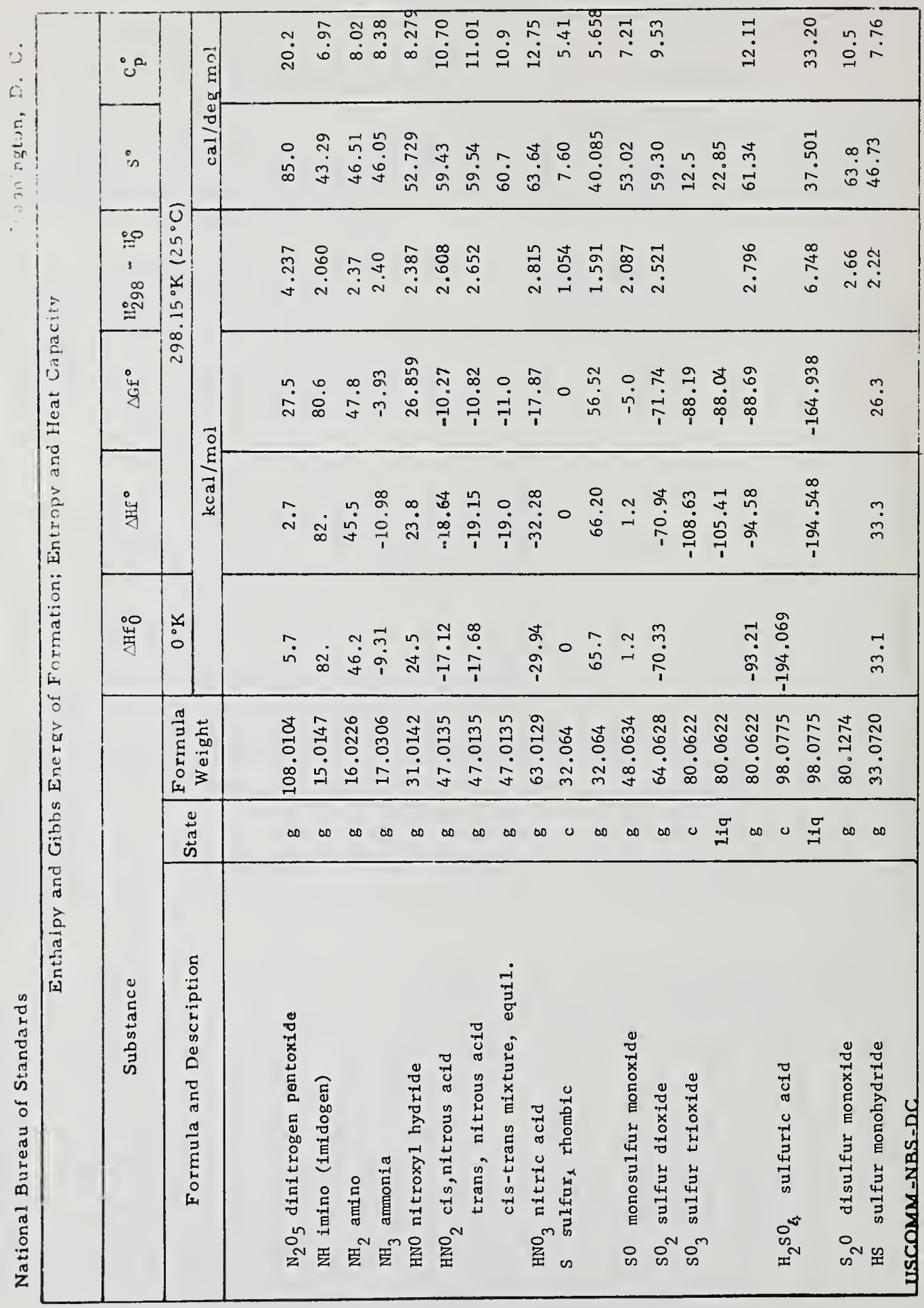


ลิ

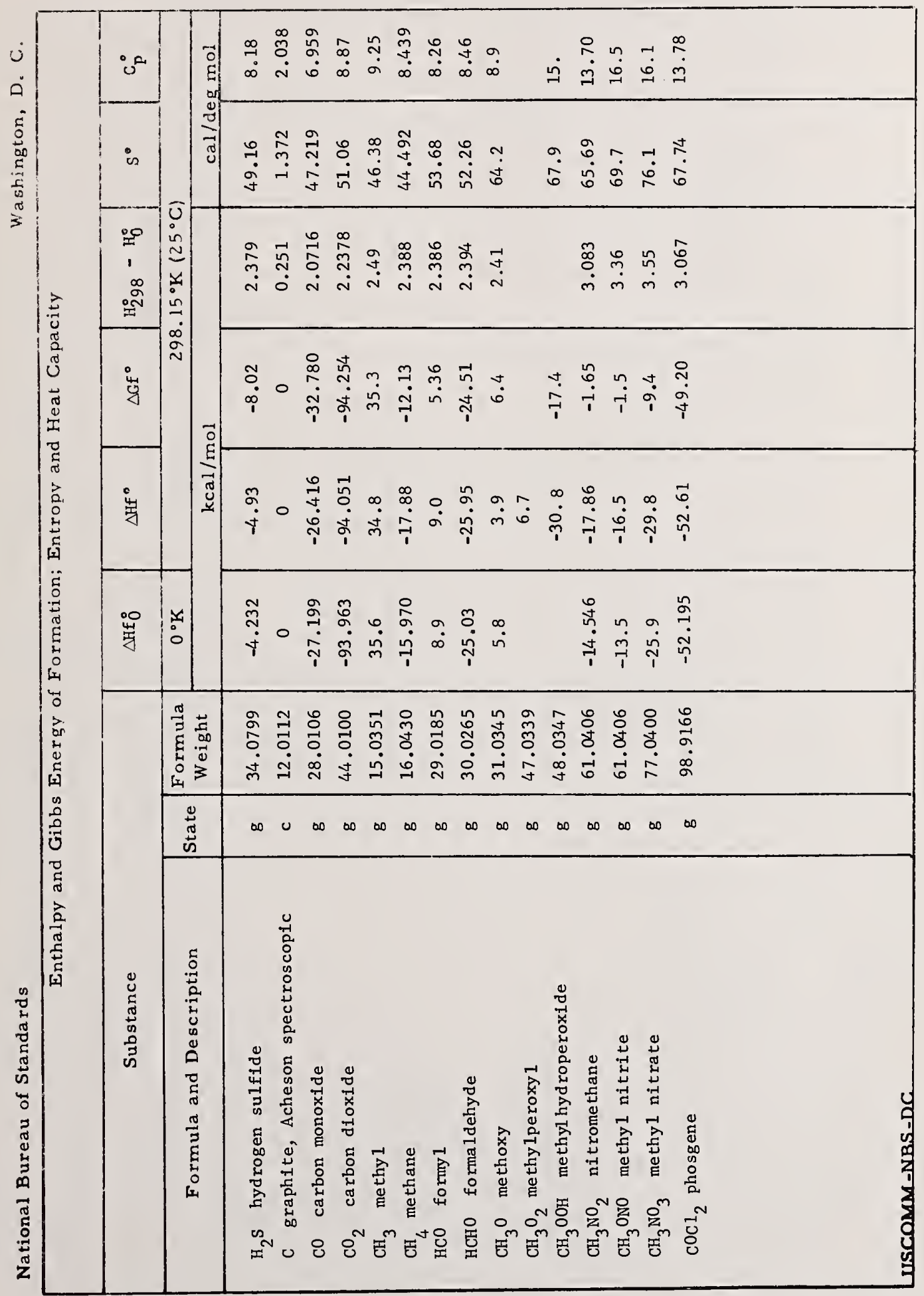




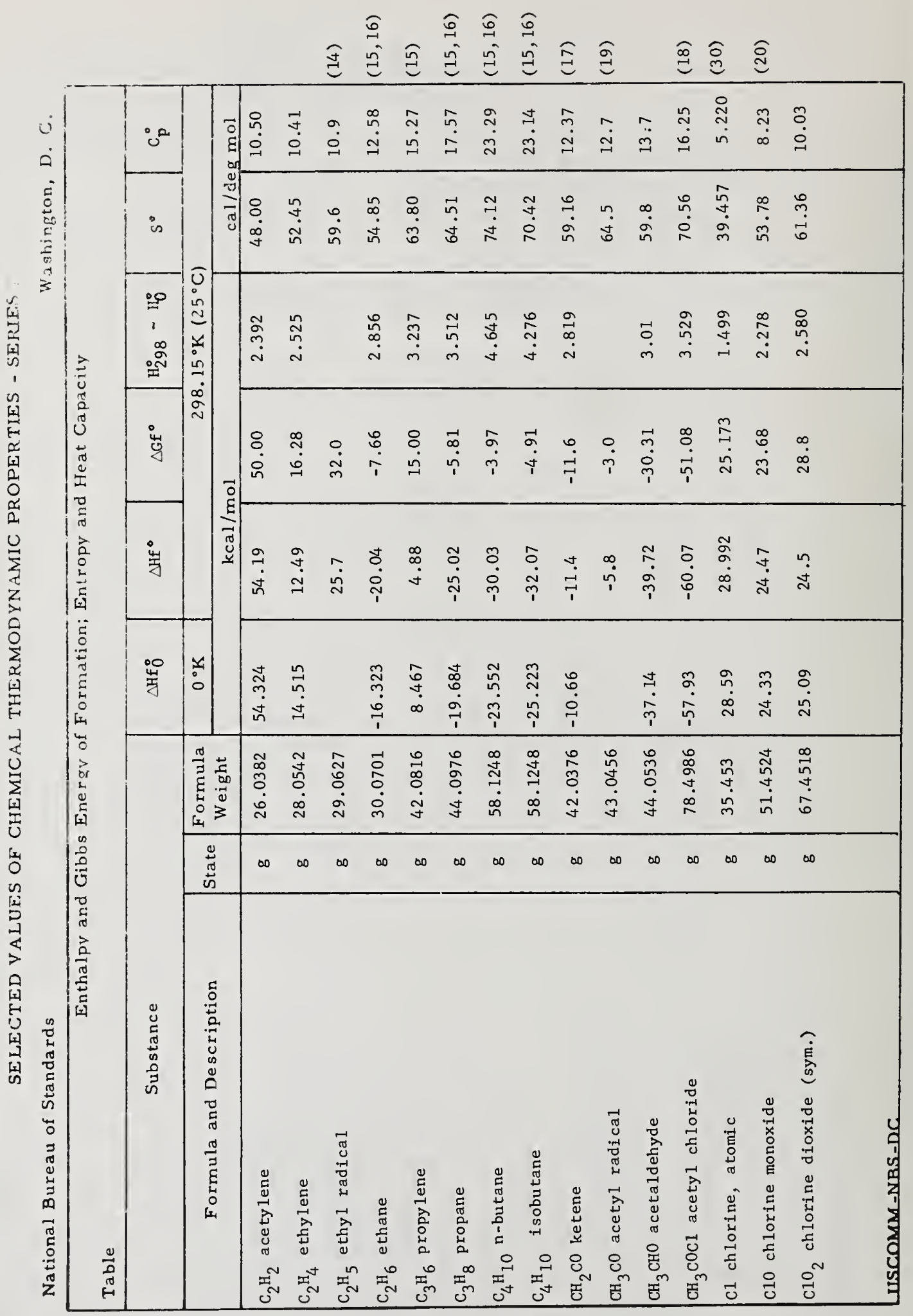




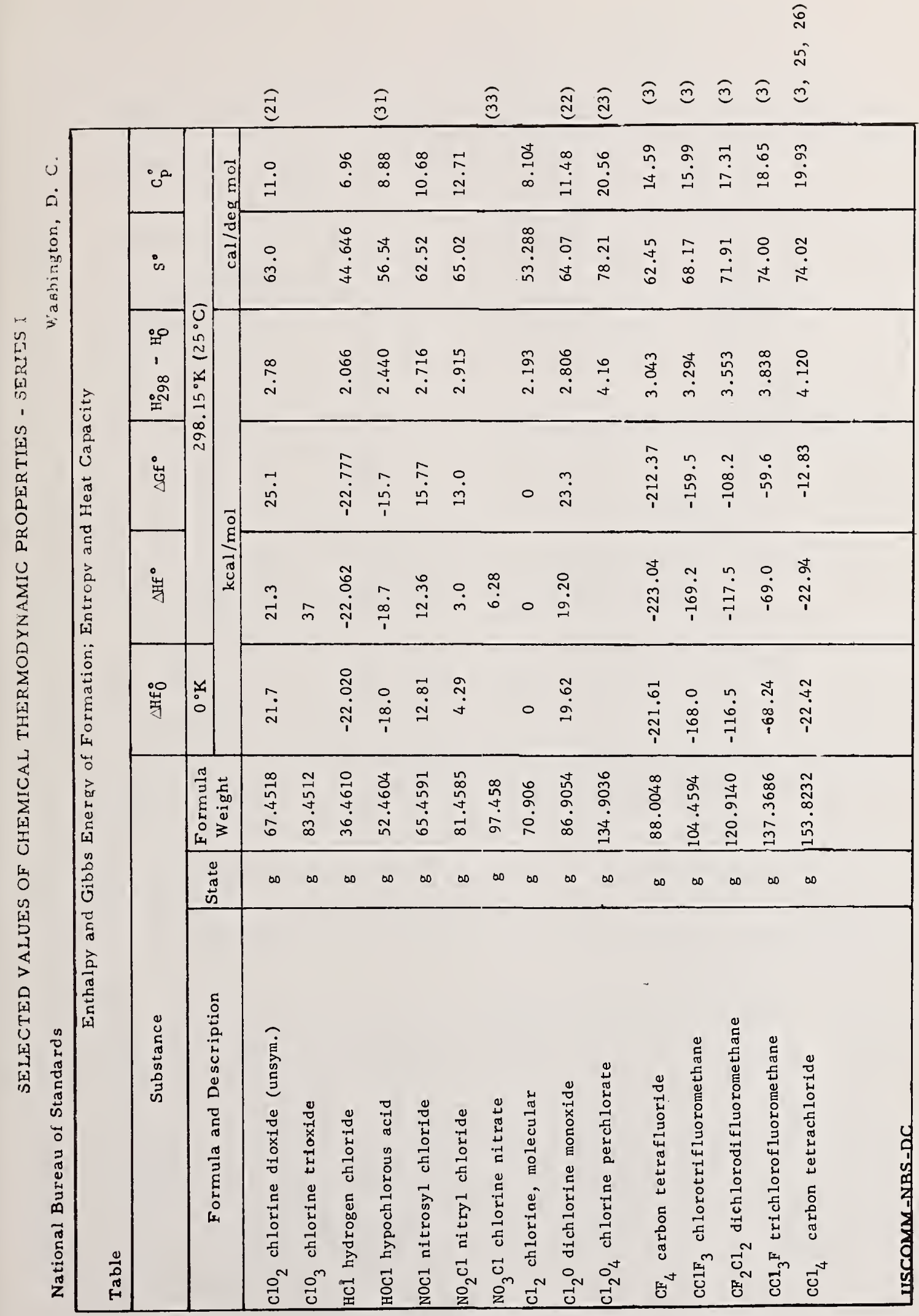




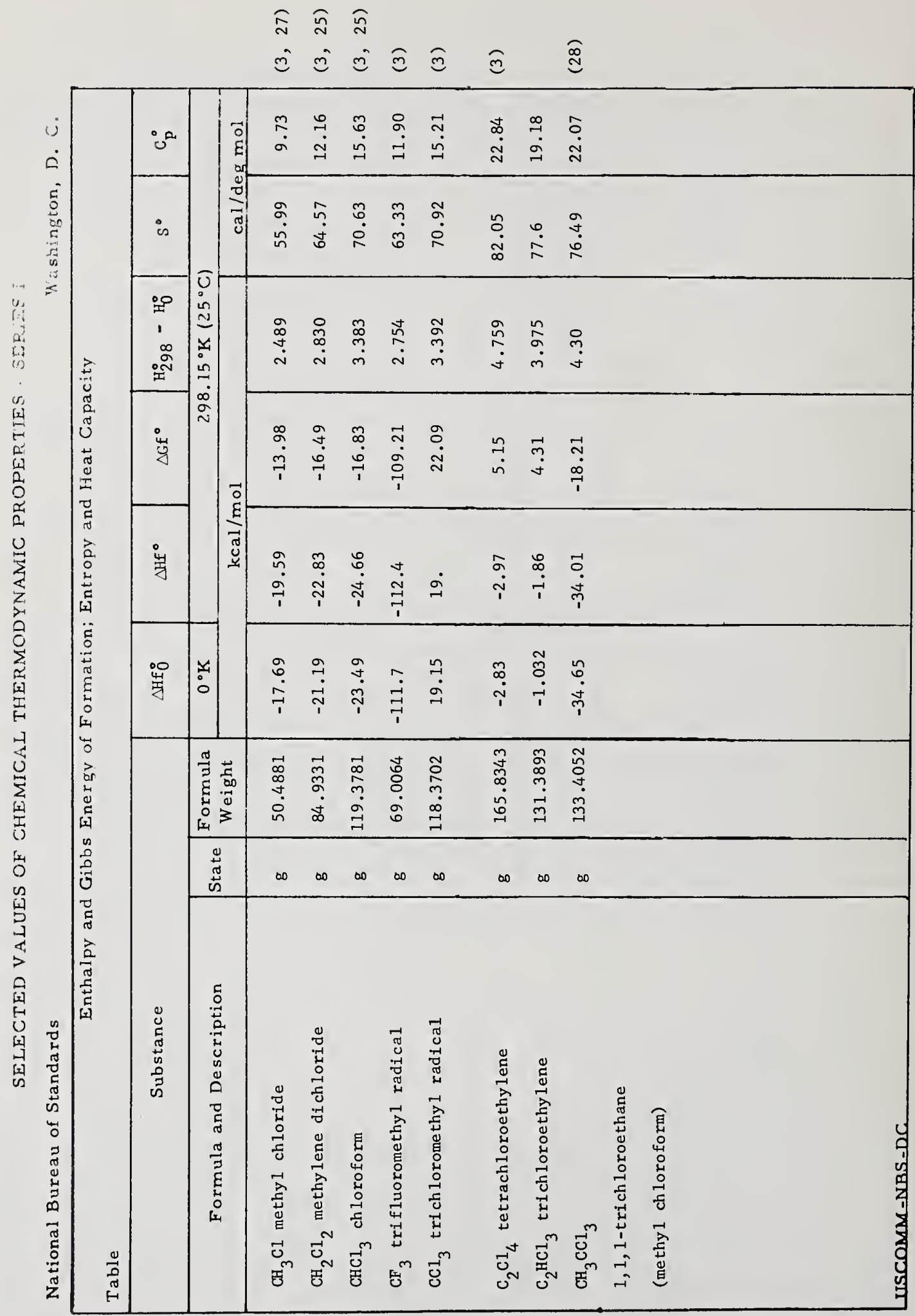




\section{Notes}

1. Hampson, R. F. et al, J. Phys. Chem. Ref. Data 2, 267-312 (1973) [Data sheet on $\mathrm{O}_{3}+$ hv (u.v.)] p. 303 .

2. Contributions of the higher electronic states of molecular oxygen to the enthalpy, entropy, and heat capacity at $298 \mathrm{~K}$ are insignificant.

3. Stull, D. R. and Prophet, H. "JANAF Thermochemical Tables" 2nd. ed. NSRDS-NBS 37 (1971).

4. Tsang, W. Estimated from kinetic data on the decomposition of $\mathrm{N}_{2} \mathrm{H}_{4}$. Chapter 12, NBS Report 10904, 1 July 1972 .

5. NBS Report 10904, 1 Ju1y 1972, pp. 239-307.

6. R. A. Fletcher and G. Pilcher, Trans Faraday Soc. 66, 794-799 (1970) provide more recent data for $\Delta \mathrm{Hf}^{\circ}, 298.15 \mathrm{~K}$.

7. Data obtained from Benson and O'Neal ("Kinetic Data on Gas Phase Unimolecular Reactions", NSRDS-NBS-21, Feb 1970, page 55).

8. Heat formation value from S. W. Benson "Thermochemical Kinetics" Second Edition, John Wiley and Sons (1976).

9. Values for $\Delta \mathrm{Hf}^{\circ}, \mathrm{S}^{\circ}$, and $\mathrm{CP}^{\circ}$ at $298.15 \mathrm{~K}$ were calculated using the group additivity method described by Benson and $0^{\prime}$ Neal ("Kinetic Data on Gas Phase Unimolecular Reactions", NSRDS-NBS21, Feb. 1970, pp. 40-42). 
10. The value for $\triangle \mathrm{Hf}^{\circ}\left[\mathrm{CH}_{3} \mathrm{ONO}(\mathrm{g})\right]=-16.5 \mathrm{kcal} \mathrm{mol}{ }^{-1}$ was derived from the combustion data of Geiseler and Thierfelder (Z. physik. Chem., N. F., 29, 248-257 (1961)), and the equilibrium data of Leermakers and Ramsperger (J. Am. Chem. Soc. 54, 1837-1845 (1932)) for: $\mathrm{CH}_{3} \mathrm{OH}(\mathrm{g})+\mathrm{NOCl}(\mathrm{g})=\mathrm{CH}_{3} \mathrm{ONO}(\mathrm{g})+\mathrm{HCl}(\mathrm{g})$.

11. The value for $\Delta \mathrm{Hf}^{\circ}\left(\mathrm{CH}_{3} \mathrm{ONO}_{2}(\mathrm{~g})\right)=-29.8 \mathrm{kcal} \mathrm{mol}{ }^{-1}$ was derived from the work of Ray and Ogg (J. Phys. Chem. 63, 1522-1523 (1959)) on the enthalpy of reaction of $\mathrm{N}_{2} \mathrm{O}_{5}$ with $\mathrm{CH}_{3} \mathrm{ONO}$.

12. We have chosen $\mathrm{S}^{\circ}\left[\mathrm{CH}_{3} \mathrm{ONO}_{2}(\mathrm{~g})\right]=76.1 \mathrm{cal} \mathrm{mol}^{-1} \mathrm{~K}^{-1}$ based upon low temperature thermal data of Gray and Smith (J. Chem. Soc. 1953, 2380-2385). Calculations from microwave studies by Dixon and Wilson (J. Chem. Phys. 35, 191-198 (1961)) give $\mathrm{S}^{\circ}=$ $72.15 \mathrm{ca} 1 \mathrm{~mol}^{-1} \mathrm{~K}^{-1}$.

13. Davies, J. V., and Pritchard, H. 0., J. Chem. Thermodynamics 4, 23-29 (1972) provide more recent $\Delta H f^{\circ}$ data.

14. Data on $\triangle H f^{\circ}, S^{\circ}, C_{p}^{\circ}$ obtained from $S . W$. Benson and $H . E$. O'Neal ("Kinetic Data on Gas Phase Unimolecular Reactions", NSRDS-NBS-21, Feb. 1970, page 567).

15. Rossini, F. D., Pitzer, K. S., Arnett, R. L., Braun, R. M. and Pimentel, G. C., "Selected Values of Physical and Thermodynamic Properties of Hydrocarbons and Related Compounds", API Research Project 44, Carnegie Press, Pittsburgh, 1953. 
16. $\Delta H f^{\circ}$ data from Pittam and Pilcher (J. Chem. Soc., Faraday Trans. I, 68, 2224-2229 (1972).

17. $\Delta H f^{\circ}$ data from R. L. Nutta11, A. H. Laufer and M. V. Kilday., J. Chem. The rmodynamics $\underline{3}, 167-174$ (1971).

18. Devore, J. A., and 0'Nea1, H. E., J. Phys. Chem. 73, 26442648 (1969).

19. Data on $\mathrm{S}^{\circ}$ and $\mathrm{C}_{\mathrm{p}}{ }^{\circ}$ were obtained from Note (14), page 589; data on $\triangle H f^{\circ}$ came from Note (18).

20. Spectroscopic studies of N. Basco and R. D. Morse, J. Molec. Spectros. 45, 35 (1973); R. A. Durie and D. A. Ramsay, Can. J. Phys. 36, 35 (1958); P. A. G. O'Hare and A. C. Wah1, J. Chem. Phys. 54, 3770 (1971); L. Andrews and J. I. Raymond, J. Chem. Phys. 55, 3087 (1971); D. R. Johnson and F. X. Powe11, Bu11. Am. Phys. Soc. 13, 594 (1968); A. Carrington, P. N. Dyer, and D. H. Levy, J. Chem. Phys. 47, 1756 (1967) were examined and used to calculate the the rmodynamic properties:

21. Arke11, A., and Schwager, I., J.Am. Chem. Soc. 89, 5999 (1967); Johnston, H., Morris, E. D., and Van den Bogaerde, J., J. Am. Chem. Soc. 91, 7712 (1969), C1yne, M. A. A. and White, I. F., Trans. Faraday Soc. 67, 2068 (1971).

22. Studies by D. J. Gardiner, J. Mo1. Spectroscopy 38, 476 (1971) and G. E. Herberich, R. H. Jackson, and D. J. Mi11er, J. Chem. Soc. (A) 1966, 336 were examined and the data used to calculate the thermodynamic properties. 
23. The spectroscopic data of K. 0. Christe, C. J. Schack and E. C. Curtis, Inorg. Chem. 10, 1589 (1971) were used.

24. Thermal Constants of Substances (Termichesky Konstanty Veshchestv), Vol. IV, V. P. Glushko, Editor, Moscow (1970).

25. The combustion data of A. T. Hu and G. C. Sinke, J. Chem. Thermodynamics $\underline{1}, 507-513$ (1969) were used to derive $\Delta H f^{\circ}$ $298.15 \mathrm{~K}$ values for $\mathrm{CCl}_{4}, \mathrm{CHCl}_{3}$, and $\mathrm{CH}_{2} \mathrm{Cl}_{2}$ in the liquid state.

26. The vaporization data of D. L. Hildenbrand and R. A. McDonald, J. Phys. Chem. 63, 1521-1522 (1959) were used.

27. The combustion data of R. A. Fletcher and G. Pilcher, Trans. Faraday Soc. 67, 3191-3201 (1971) were used to calculate $\Delta \mathrm{Hf}^{\circ}\left[\mathrm{CH}_{3} \mathrm{Cl}(\mathrm{g})\right]$.

28. J. Chao, A. S. Rodgers, R. C. Wilhoit, and B. J. Zwolinski, J. Phys. \& Chem. Ref. Data 3 , 141-162 (1974) provide a recent evaluation of the thermodynamic properties of $\mathrm{CH}_{3} \mathrm{CCl}_{3}$.

29. Based on data in H. Okabe and M. Lenzi, J. Chem. Phys. 47, 5241 (1967) and in J. Masanet, A. Gilles and C. Vermeil, J. Photochemistry $\underline{3}, 417(1974 / 75)$.

30. Parker, V. B., Wagman, D. D., and Garvin, D., NBSIR 75-968. Selected thermochemical data compatible with the CODATA recommendations, January 1976.

31. Value for heat of formation derived from equilibrium studies reported by H. Imagawa, Denki Kagaku 19, 271 (1951). 
32. JANAF Thermochemical Tables Supplement No. 48, June 1977.

33. Heat of formation value based on data in H. D. Knauth, H. Martin and W. Stockmann, Z. Naturforsch. 29A, 200 (1974).

34. Wagman, D.D. Private communication, 
Bond Dissociation Energies, $\mathrm{D}_{\mathrm{O}}^{\mathrm{O}}$ at $0 \mathrm{~K}$

Calculated from heats of formation in the accompanying table

\begin{tabular}{|c|c|c|c|}
\hline Reaction & $\mathrm{kJ} / \mathrm{mol}$ & $\mathrm{kcal} / \mathrm{mol}$ & Note \\
\hline $\mathrm{O}_{2} \rightarrow 20$ & $493.59 \pm 0.4$ & $117.97 \pm 0.1$ & \\
\hline $\mathrm{O}_{3} \rightarrow \mathrm{O}_{2}+\mathrm{O}$ & $101.4 \pm 2.1$ & $24.24 \pm 0.5$ & \\
\hline $\mathrm{H}_{2} \rightarrow 2 \mathrm{H}$ & $432.00 \pm 0.04$ & $103.25 \pm 0.01$ & \\
\hline $\mathrm{HO} \rightarrow \mathrm{H}+\mathrm{O}$ & $423.8 \pm 2.1$ & $101.3 \pm 0.5$ & \\
\hline $\mathrm{HO}_{2} \rightarrow \mathrm{HO}+\mathrm{O}$ & $230 \pm 8$ & $67 \pm 2$ & \\
\hline$\rightarrow \mathrm{H}+\mathrm{O}_{2}$ & $210 \pm 8$ & $50 \pm 2$ & \\
\hline $\mathrm{H}_{2} \mathrm{O} \rightarrow \mathrm{HO}+\mathrm{H}$ & $493.7 \pm 0.8$ & $118.0 \pm 0.2$ & \\
\hline $\mathrm{H}_{2} \mathrm{O}_{2} \rightarrow 2 \mathrm{HO}$ & $207.1 \pm 2.1$ & $49.5 \pm 0.5$ & \\
\hline $\mathrm{H}_{2} \mathrm{O}_{2} \rightarrow \mathrm{H}+\mathrm{HO}_{2}$ & $350 \pm 8$ & $84 \pm 2$ & \\
\hline $\mathrm{N}_{2} \rightarrow \mathrm{N}+\mathrm{N}$ & $941.69 \pm 0.04$ & $225.07 \pm 0.01$ & \\
\hline $\mathrm{NO} \rightarrow \mathrm{N}+\mathrm{O}$ & 627.9 & 150.1 & \\
\hline $\mathrm{NO}_{2} \rightarrow \mathrm{NO}+\mathrm{O}$ & 300.5 & 71.83 & \\
\hline $\mathrm{NO}_{3} \rightarrow \mathrm{NO}_{2}+\mathrm{O}$ & $205 \pm 21$ & $49 \pm 5$ & \\
\hline $\mathrm{N}_{2} \mathrm{O} \rightarrow \mathrm{N}+\mathrm{NO}$ & $474.9 \pm 4$ & $113.5 \pm 1$ & \\
\hline$\rightarrow \mathrm{N}_{2}+\mathrm{O}$ & 161.3 & 38.58 & \\
\hline $\mathrm{N}_{2} \mathrm{O}_{3} \rightarrow \mathrm{NO}+\mathrm{NO}_{2}$ & $35.1 \pm 0.8$ & $8.4 \pm 0.2$ & \\
\hline $\mathrm{N}_{2} \mathrm{O}_{4} \rightarrow \mathrm{NO}_{2}+\mathrm{NO}_{2}$ & 53.2 & 12.7 & \\
\hline $\mathrm{N}_{2} \mathrm{O}_{5} \rightarrow \mathrm{NO}_{2}+\mathrm{NO}_{3}$ & 89.5 & 21.4 & \\
\hline $\mathrm{HNO} \rightarrow \mathrm{H}+\mathrm{NO}$ & 203 & 48.6 & \\
\hline$\rightarrow \mathrm{NH}+\mathrm{O}$ & 487 & 116.5 & \\
\hline trans $-\mathrm{HNO}_{2} \rightarrow \mathrm{HO}+\mathrm{NO}$ & 202 & 48.4 & \\
\hline $\mathrm{HNO}_{3} \rightarrow \mathrm{HO}+\mathrm{NO}_{2}$ & 200 & 47.8 & \\
\hline $\mathrm{NH} \rightarrow \mathrm{H}+\mathrm{N}$ & 343 & 82 & \\
\hline
\end{tabular}


Bond Dissociation Energies, $D_{0}^{0}$ at $0 \mathrm{~K}$

Calculated from heats of formation in the accompanying table

\begin{tabular}{llll}
\hline \multicolumn{1}{c}{ Reaction } & \multicolumn{1}{c}{$\mathrm{kJ} / \mathrm{mol}$} & $\mathrm{kcal} / \mathrm{mol}$ & Note \\
\hline $\mathrm{NH}_{2} \rightarrow \mathrm{H}+\mathrm{HN}$ & 366 & 87.4 \\
$\mathrm{NH}_{3} \rightarrow \mathrm{NH}_{2}+\mathrm{H}$ & 448 & 107 \\
$\mathrm{CO} \rightarrow \mathrm{C}+\mathrm{O}$ & $1071.94 \pm 0.4$ & $256.2 \pm 0.1$ \\
$\mathrm{CO}_{2} \rightarrow \mathrm{CO}+\mathrm{O}$ & $525.9 \pm 0.4$ & $125.7 \pm 0.1$ \\
$\mathrm{CHO}^{\rightarrow \mathrm{CO}+\mathrm{H}}$ & 70 & 16.7 \\
$\mathrm{CH}_{2} \mathrm{O} \rightarrow \mathrm{CHO}+\mathrm{H}$ & 364 & 87 \\
$\mathrm{CH}_{3} \mathrm{O} \rightarrow \mathrm{CH}_{3}+\mathrm{O}$ & 378 & 90.4 \\
$\quad \rightarrow \mathrm{H}+\mathrm{H}_{2} \mathrm{CO}$ & 93 & 22 \\
$\mathrm{CH}_{3} \mathrm{O}{ }_{2} \rightarrow \mathrm{CH}_{3} \mathrm{O}+\mathrm{O}$ & 237 & 56.8 \\
$\quad \rightarrow \mathrm{CH}_{3}+\mathrm{O}_{2}$ & 118 & 28.1
\end{tabular}

Note 1. Calculated for $T=298 \mathrm{~K}$ 


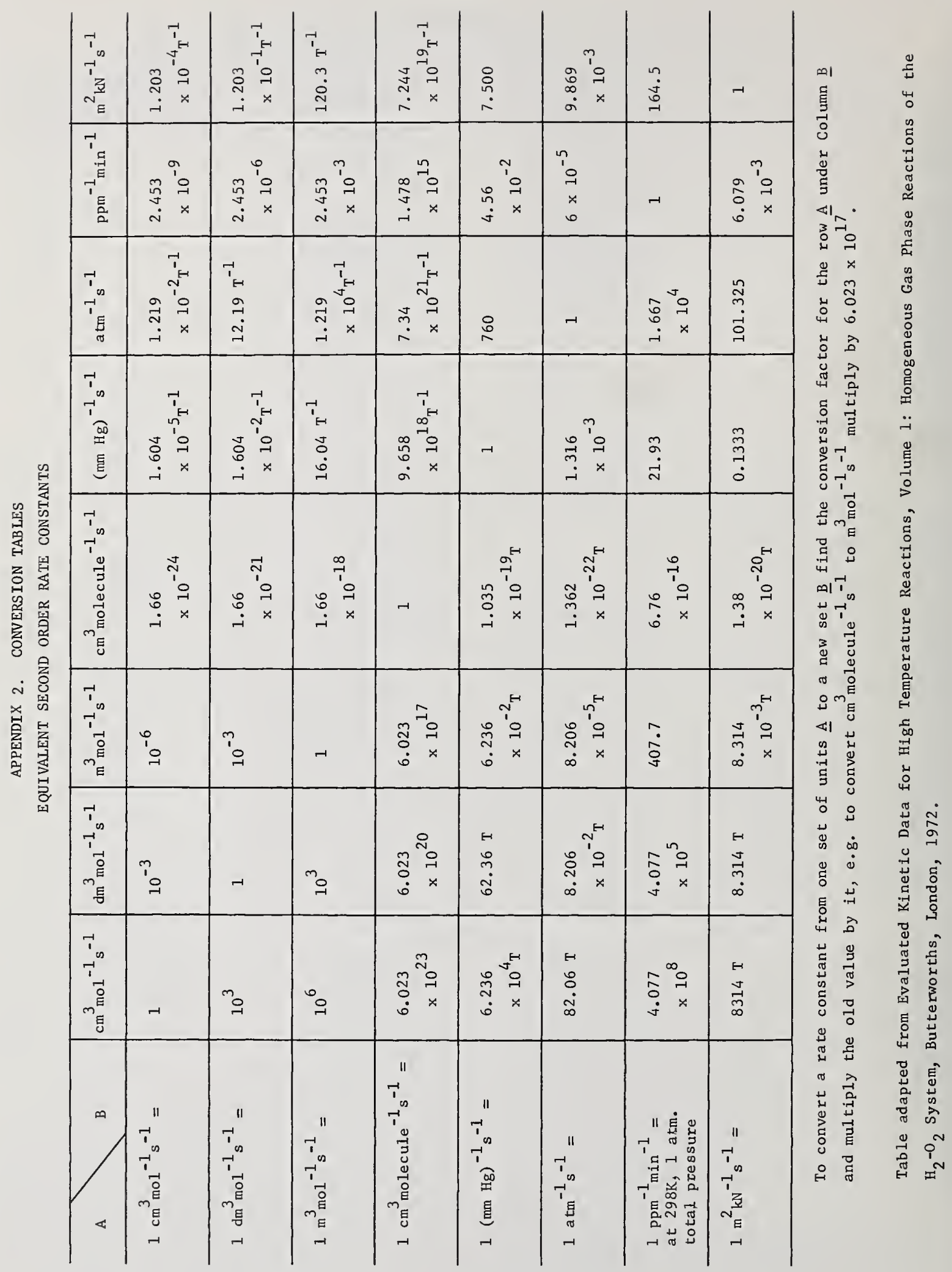




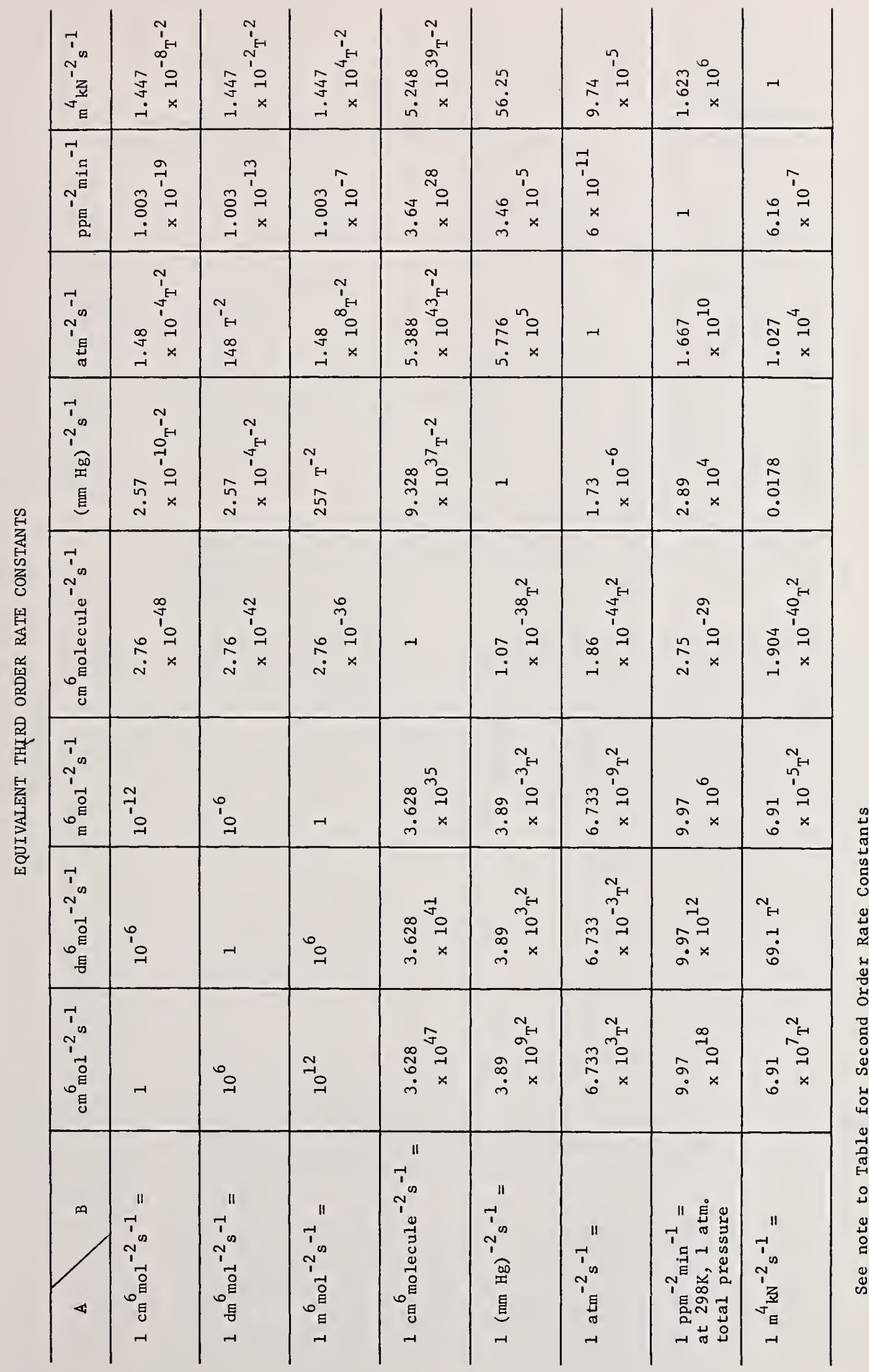




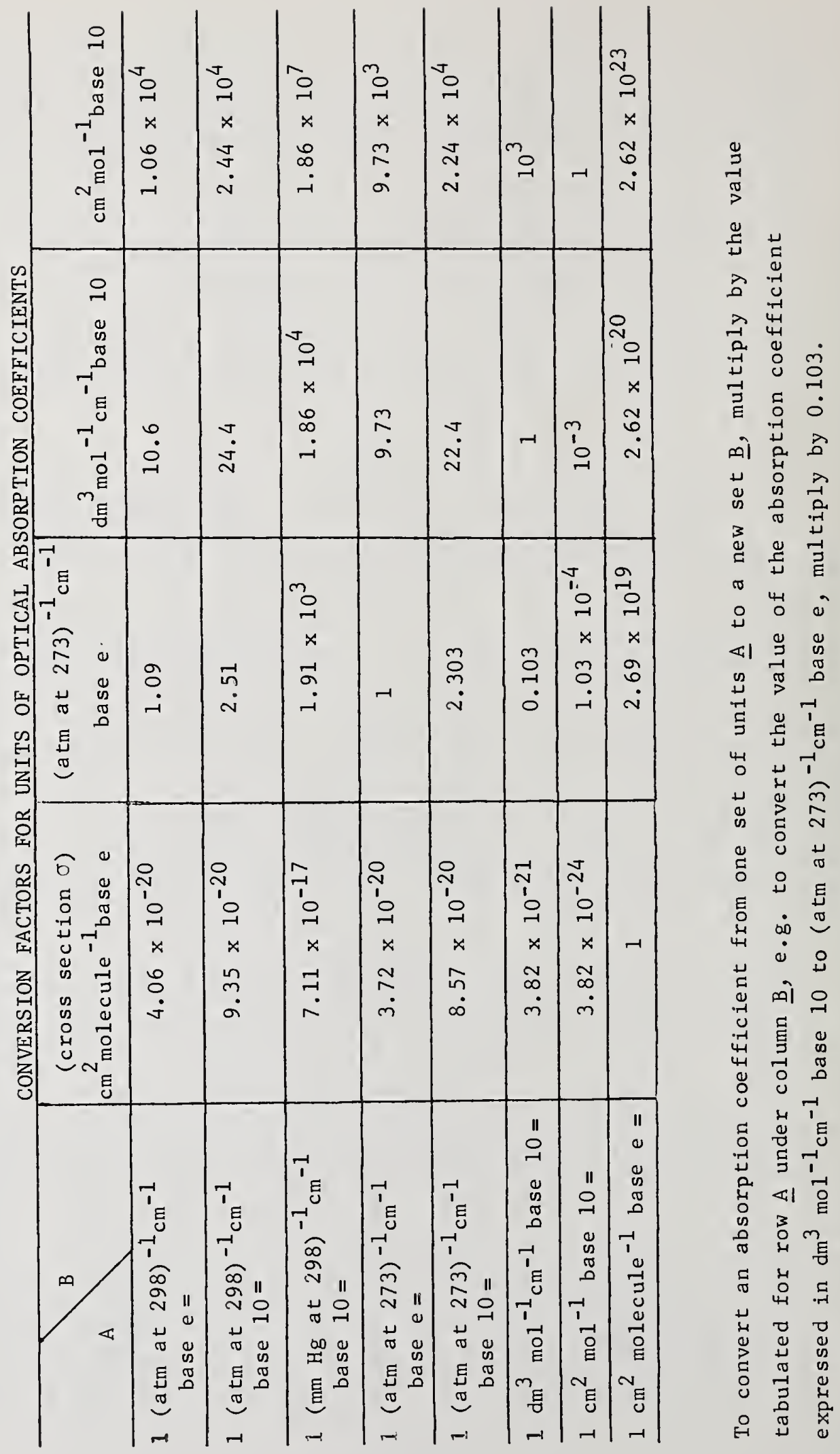


NBS-114A (REV. 7-73)

\begin{tabular}{|c|c|c|c|}
\hline $\begin{array}{l}\text { U.S. DEPT. OF COMM. } \\
\text { BIBLIOGRAPHIC DATA } \\
\text { SHEET }\end{array}$ & $\begin{array}{l}\text { 1. PUBLICATION OR REPORT NO. } \\
\text { NBS SP-513 }\end{array}$ & $\begin{array}{l}\text { 2. Gov't Accession } \\
\text { No. }\end{array}$ & 3. Recipient's Accession No. \\
\hline \multicolumn{3}{|c|}{$\begin{array}{l}\text { 4. TITLE AND SUBTITLE } \\
\text { Reaction Rate and Photochemical Data for } \\
\text { Atmospheric Chemistry - } 1977\end{array}$} & $\begin{array}{l}\text { 5. Publication Date } \\
\text { May } 1978 \\
\text { 6. Performing Organization Code }\end{array}$ \\
\hline \multicolumn{3}{|c|}{$\begin{array}{l}\text { 7. AUTHOR(S) } \\
\text { Robert F. Hampson, Jr., and David Garvin, Editors }\end{array}$} & 8. Performing Organ. Report No. \\
\hline \multicolumn{3}{|c|}{$\begin{array}{l}\text { 9. PERFORMING ORGANIZATION NAME AND ADDRESS } \\
\text { NATIONAL BUREAU OF STANDARDS } \\
\text { DEPARTMENT OF COMMERCE } \\
\text { WASHINGTON, D.C. } 20234\end{array}$} & 10. Project/Task/Work Unit No. \\
\hline \multicolumn{3}{|c|}{$\begin{array}{l}\text { 12. Sponsoring Organization Name and Complete Address (Street, City, State, ZIP) } \\
\text { Department of Transportation, FAA, Washington, DC } 20591 \\
\text { N.A.S.A., Washington, DC 20546 } \\
\text { Office of Standard Reference Data, NBS, Washington, DC } 20234 \\
\text { Office of Environmental Measurements, NBS, Washington DC } 20234\end{array}$} & $\begin{array}{l}\text { 13. Type of Report \& Period } \\
\text { Covered } \\
\text { 14. Sponsoring Agency Code }\end{array}$ \\
\hline
\end{tabular}

Library of Congress Catalog Card Number: 78-606033

16. ABSTRACT (A 200-word or less factual summary of most significant information. If document includes a significant bibliography or literature survey, mention it here.)

A table of data for gas phase chemical reactions and photochemistry of neutral species is presented. Specifically, it gives preferred values for reaction rate constants, photoabsorption cross sections, and quantum yields of primary photochemical processes and also cites recent experimental work (to December 1977). It is intended to provide the basic physical chemical data needed as input data for calculations modelling atmospheric chemistry. An auxiliary table of thermochemical data for the pertinent chemical species is given in the appendix.

17. KEY WORDS (six to twelve entries; alphabetical order; capitalize only the first letter of the first key word unless a proper name; separated by semicolons) Air pollution; atmospheric chemistry; chemical kinetics; data evaluation; gas phase; photoabsorption cross section; photochemistry; quantum yield; rate constant

\section{AVAILABILITY \\ $\mathrm{x}$ Unlimited}

For Official Distribution. Do Not Release to NTIS

X Order From Sup. of Doc., U.S. Government Printing Office Washington, D.C. 20402, SD Stock No. SN003-003

Order From National Technical Information Service (NTIS) Springfield, Virginia 22.151

\begin{tabular}{|l|c|}
$\begin{array}{l}\text { 19. SECURITY CLASS } \\
\text { (THIS REPURT) }\end{array}$ & 21. NO. OF PAGES \\
$\begin{array}{l}\text { UNCL ASSIFIED } \\
\begin{array}{l}\text { 20. SECURITY CLASS } \\
\text { (THIS PAGE) }\end{array} \\
\text { UNCLASSIFIED }\end{array}$ & 22. Price \\
\hline
\end{tabular}





\section{PERIODICALS}

JOURNAL OF RESEARCH-The Journal of Research of the National Bureau of Standards reports NBS research and development in those disciplines of the physical and engineering sciences in which the Bureau is active. These include physics, chemistry, engineering, mathematics, and computer sciences. Papers cover a broad range of subjects, with major emphasis on measurement methodology, and the basic technology underlying standardization. Also included from time to time are survey articles on topics closely related to the Bureau's technical and scientific programs. As a special service to subscribers each issue contains complete citations to all recent NBS publications in NBS and nonNBS media. Issued six times a year. Annual subscription: domestic $\$ 17.00$; foreign $\$ 21.25$. Single copy, $\$ 3.00$ domestic; $\$ 3.75$ foreign.

Note: The Journal was formerly published in two sections: Section A "Physics and Chemistry" and Section B "Mathematical Sciences."

\section{DIMENSIONS/NBS}

This monthly magazine is published to inform scientists, engineers, businessmen, industry, teachers, students, and consumers of the latest advances in science and technology, with primary emphasis on the work at NBS. The magazine highlights and reviews such issues as energy research, fire protection, building technology, metric conversion, pollution abatement, health and safety, and consumer product performance. In addition, it reports the results of Bureau programs in measurement standards and techniques, properties of matter and materials, engineering standards and services, instrumentation, and automatic data processing.

Annual subscription: Domestic, \$12.50; Foreign $\$ 15.65$.

\section{NONPERIODICALS}

Monographs-Major contributions to the technical literature on various subjects related to the Bureau's scientific and technical activities.

Handhooks-Recommended codes of engineering and industrial practice (including safety codes) developed in cooperation with interested industries, professional organizations, and regulatory bodies.

Special Puhlications-Include proceedings of conferences sponsored by NBS, NBS annual reports, and other special publications appropriate to this grouping such as wall charts, pocket cards, and bibliographies.

Applied Mathematics Series-Mathematical tables, manuals, and studies of special interest to physicists, engineers, chemists, biologists, mathematicians, computer programmers, and others engaged in scientific and technical work.

National Standard Reference Data Series-Provides quantitative data on the physical and chemical properties of materials, compiled from the world's literature and critically evaluated. Developed under a world-wide program coordinated by NBS. Program under authority of National Standard Data Act (Public Law 90-396).
NOTE: At present the principal publication outlet for these data is the Journal of Physical and Chemical Reference Data (JPCRD) published quarterly for NBS by the American Chemical Society (ACS) and the American Institute of Physics (AIP). Subscriptions, reprints, and supplements available from ACS, 1155 Sixteenth St. N.W., Wash., D.C. 20056.

Building Science Series-Disseminates technical information developed at the Bureau on building materials, components, systems, and whole structures. The series presents research results, test methods, and performance criteria related to the structural and environmental functions and the durability and safety characteristics of building elements and systems. Technical Notes-Studies or reports which are complete in themselves but restrictive in their treatment of a subject. Analogous to monographs but not so comprehensive in scope or definitive in treatment of the subject area. Often serve as a vehicle for final reports of work performed at NBS under the sponsorship of other government agencies. Voluntary Product Standards-Developed under procedures published by the Department of Commerce in Part 10, Title 15, of the Code of Federal Regulations. The purpose of the standards is to establish nationally recognized requirements for products, and to provide all concerned interests with a basis for common understanding of the characteristics of the products. NBS administers this program as a supplement to the activities of the private sector standardizing organizations.

Consumer Information Series-Practical information, based on NBS research and experience, covering areas of interest to the consumer. Easily understandable language and illustrations provide useful background knowledge for shopping in today's technological marketplace.

Order above NBS publications from: Superintendent of Documents, Government Printing Office, Washington, D.C. 20402.

Order following NBS publications--NBSIR's and FIPS from the National Technical Information Services, Springfield, Va. 22161.

Federal Information Processing Standards Publications (FIPS PUB)-Publications in this series collectively constitute the Federal Information Processing Standards Register. Register serves as the official source of information in the Federal Government regarding standards issued by NBS pursuant to the Federal Property and Administrative Services Act of 1949 as amended, Public Law 89-306 (79 Stat. 1127), and as implemented by Executive Order 11717 (38 FR 12315, dated May 11, 1973) and Part 6 of Title 15 CFR (Code of Federal Regulations).

NBS Interagency Reports (NBSIR)-A special series of interim or final reports on work performed by NBS for outside sponsors (both government and non-government). In general, initial distribution is handled by the sponsor; public distribution is by the National Technical Information Services (Springfield, Va. 22161) in paper copy or microfiche form.

\section{BIBLIOGRAPHIC SUBSCRIPTION SERVICES}

The following current-awareness and literature-survey bibliographies are issued periodically by the Bureau:

Cryogenic Data Center Current Awareness Service. A literature survey issued biweekly. Annual subscription: Domestic, $\$ 25.00$; Foreign, $\$ 30.00$.

Liquified Natural Gas. A literature survey issued quarterly. Annual subscription: $\$ 20.00$.
Superconducting Devices and Materials. A literature survey issued quarterly. Annual subscription: $\$ 30.00$. Send subscription orders and remittances for the preceding bibliographic services to National Bureau of Standards, Cryogenic Data Center (275.02) Boulder, Colorado 80302. 
U.S. HEPARTMENT OF COMMERCE

National Bureau of Standards

Washington, D.C. 20234

DFFICIAL BUSINESS

U.S. DEPARTMENT OF COMMERCE $C O M-215$

Penalty for Private Use, $\$ 300$

SPECIAL FOURTH-CLASS RATE BOOK 